Studies on Non-Prioritized

Multiple Belief Revision

Fillipe Manoel Xavier Resina

Thesis PRESENTED TO THE

Institute of MATHEMATiCs AND StATistics

of THE University of SÃo PaUlo

IN PARTIAL FULFILLMENT

OF THE REQUIREMENTS

FOR THE DEGREE OF

Doctor of SCIENCE

Program: Computer Science

Advisor: Prof. Dr. Renata Wassermann

This study was financed by the Brazilian funding agency CAPES

São Paulo

October 20, 2021 



\title{
Studies on Non-Prioritized Multiple Belief Revision
}

\author{
Fillipe Manoel Xavier Resina
}

\begin{abstract}
This version of the thesis includes the corrections and modifications suggested by the Examining Committee during the defense of the original version of the work, which took place on October 20, 2021.

A copy of the original version is available at the Institute of Mathematics and Statistics of the University of São Paulo.
\end{abstract}

Examining Committee:

Prof. Dr. Renata Wassermann (advisor) - IME-USP

Prof. Dr. Marcelo Finger - IME-USP

Prof. Dr. Eduardo Fermé - Univ. da Madeira

Prof. Dr. Marcelo Coniglio - Unicamp

Prof. Dr. Marlo Souza - UFBA 
Autorizo a reprodução e divulgação total ou parcial deste trabalho, por qualquer meio convencional ou eletrônico, para fins de estudo e pesquisa, desde que citada a fonte. 


\section{Acknowledgements}

Although a $\mathrm{PhD}$ thesis is supposed to be an individual work, it would not be achieved without the help and support from many people.

First of all, I would like to thank Jesus Christ, the Author of life, my Savior and "in whom are hidden all the treasures of wisdom and knowledge ${ }^{1}$." He is my daily strength and joy, and the One that makes everything possible. I will never have enough words to express my gratitude.

I also thank my wife Evelise for being with me and for encouraging me throughout this unique period. I thank her for coming to São Paulo in order to make this job possible, for helping me in the tough moments and for the smile and love that always make everything easier. It is with her and our children that I will have the pleasure of enjoying the fruits of this work. Still about family, I thank my parents for supporting me in the decision to start this $\mathrm{PhD}$, not only emotionally but financially as well. I thank them as well for always rooting for me. I still remember how moved my mom felt when she heard about my good grade on a difficult exam (she prayed a lot for me!) and how touched my dad was when he heard that I solved a final challenging point of my research. These moments make all the difference!

On the academic side, this work would have been impossible without the supervision and wise directions of my advisor, prof. Renata Wassermann. I thank her for leaving the doors open since the end of my Masters, for always believing in me and for the great effort in assisting me every time I requested. Still within the university environment, I thank my colleagues from LIAMF lab for the aggregating discussions, for the questions I could share and also for the lunches, laughs and for the relaxed moments that help us to take a deep breath to continue the hard work. Although the pandemic forced us to give up our daily face-to-face contact for the last year and a half, our weekly online meeting helped us a lot to share the advances and, at the same time, the uncertainties of this time.

\footnotetext{
${ }^{1}$ Holy Bible (NIV) - Colossians 2:3
} 
Also, I acknowledge the financial support from CAPES. The scholarships make it possible to keep on studying after graduation.

Finally, I thank all my relatives and friends, who have always been caring, welcoming and understanding, even when I needed to be away during difficult times. Thank you very much! 


\section{Resumo}

Fillipe Manoel Xavier Resina. Studies on Non-Prioritized Multiple Belief Revision. Tese (Doutorado). Instituto de Matemática e Estatística, Universidade de São Paulo, São Paulo, 2021.

A área de Revisão de Crenças lida com o problema de como um agente racional deve proceder ao se deparar com uma nova informação. No paradigma AGM, o framework mais utilizado em Revisão de Crenças, dado um conjunto de crenças, há três possíveis mudanças epistêmicas em relação a uma nova crença: expansão, contração e revisão. Entre elas, estamos particularmente interessados na última. Uma revisão ocorre quando um agente recebe uma nova informação possivelmente inconsistente com seu estado epistêmico e tem que mudá-lo para acomodar a nova crença de forma consistente. Entretanto, uma nova informação pode vir como um conjunto de crenças (ao invés de uma única), um problema conhecido como Revisão Múltipla, no qual, ao contrário de Revisão Iterada, todas as novas crenças são processadas simultaneamente.

Esta tese começa com um survey acerca do tema de Revisão Múltipla. A proposta é trazer e organizar o estado-da-arte da área, mostrando as diferentes abordagens desenvolvidas desde 1988 e os problemas que ainda estão em aberto.

Depois disso, são propostas diferentes contribuições em Revisão Múltipla nãopriorizada. Uma das principais propriedades na teoria AGM é o postulado de sucesso, o qual garante que uma nova informação é sempre aceita por um agente racional, mesmo quando ele tem que desistir de alguma crença razoável que ele tinha anteriormente. Porém, em cenários mais realistas, quando um agente está lidando com uma nova crença que contradiz crenças prévias ele tem a opção de rejeitá-la, uma abordagem denominada revisão não-priorizada. Entre as possíveis operações para essa abordagem, revisão choice é aquela que lida com o caso no qual é suficiente para o agente absorver apenas um subconjunto do conjunto de entrada. Nós propomos uma caracterização axiomática de revisão múltipla choice por meio de duas construções diferentes: uma baseada em conjuntos resíduos e 
outra em conjuntos kernel, juntamente com algoritmos para ambas. A abordagem foi desenvolvida para bases de crenças e não é limitada à lógica proposicional clássica.

Ainda sobre as possíveis operações para revisão múltipla não-priorizada e a opção que o agente tem de aceitar ou rejeitar uma nova informação, Revisão Seletiva surgiu como uma terceira possibilidade, permitindo ao agente aceitar apenas uma parte da nova crença. A operação foi definida inicialmente para entradas de uma única sentença e para conjuntos de crenças. Esta tese propõe uma generalização de Revisão Seletiva para o caso múltiplo, tanto para conjuntos de crenças (teorias) quanto para bases de crença. Nós fornecemos construções, postulados e teoremas de representação para diferentes classes de Revisão Seletiva Múltipla. 


\section{Abstract}

Fillipe Manoel Xavier Resina. Studies on Non-Prioritized Multiple Belief Revision. Thesis (Doctorate). Institute of Mathematics and Statistics, University of São Paulo, São Paulo, 2021.

Belief Revision deals with the problem of how a rational agent should proceed in face of new information. In the AGM paradigm, the most used framework in Belief Revision, given a set of beliefs, there are three possible epistemic changes in relation to a new belief: expansion, contraction and revision. Among them, we are particularly interested in the latter. A revision occurs when an agent receives new information possibly inconsistent with its epistemic state and has to change it in order to accommodate the new belief in a consistent way. However, new information may come as a set of beliefs (instead of a single one), a problem known as Multiple Revision, in which, unlike Iterated Revision, all new pieces of information are processed simultaneously.

This thesis starts with a survey on the topic of Multiple Revision. The purpose is to bring and organize the state-of-the-art of the area, showing the different approaches developed since 1988 and the open problems that still exist.

After that, different contributions on non-prioritized multiple revision are proposed. One of AGM revision's main properties is success, which guarantees that new information is always accepted by a rational agent, even when it has to give up a reasonable belief previously held. However, in more realistic scenarios, when dealing with a new belief that contradicts previous ones, an agent has the option to reject it, an approach called non-prioritized revision. Among the possible operations for this approach, Choice Revision is the one that deals with the case in which it is enough for the agent to absorb only a subset of the input set. We propose an axiomatic characterization of Choice Multiple Revision through two different constructions: one based on remainder sets and the other on kernel sets, along with algorithms for both of them. The approach was developed for belief bases and is not restricted to classical propositional logic. 
Still about the possible operations for non-prioritized multiple revision and the option the agent has to accept or reject a new information, Selective Revision came up as a third possibility, allowing the agent to accept only a part of the new belief. The operation was initially defined for single sentences as inputs and for belief sets. This thesis proposes a generalization of Selective Revision to the multiple case for both belief sets (theories) and belief bases. We provide constructions, postulates and representation theorems for different classes of Multiple Selective Revision. 


\section{Contents}

1 Introduction 1

1.1 Multiple Revision . . . . . . . . . . . . . . . . . . . . 1

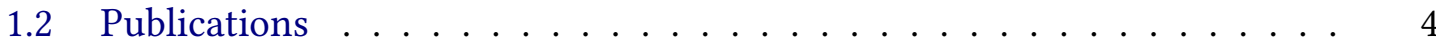

1.3 Organization of the Thesis . . . . . . . . . . . . . . 4

2 Beginnings of Revision $\quad 7$

2.1 Notation . . . . . . . . . . . . . . . . . 7

2.2 Epistemic States . . . . . . . . . . . . . . . . . . . 8

2.3 Epistemic Attitudes . . . . . . . . . . . . . . . . . 8

2.4 The Revision Operation . . . . . . . . . . . . . . . . . . . 9

2.5 Properties . . . . . . . . . . . . . . . . . . 10

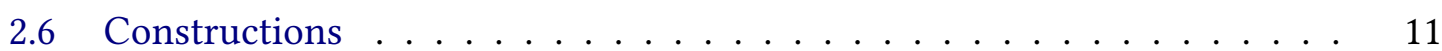

2.6.1 Partial Meet Contraction . . . . . . . . . . . . . . . . 11

2.6.2 Partial Meet Revision . . . . . . . . . . . . . . . . . . . . . 12

2.6.2.1 Working with Belief Bases . . . . . . . . . . . 13

2.6.3 Kernel Contraction . . . . . . . . . . . . . . . . . . . 15

2.6.4 Kernel Revision . . . . . . . . . . . . . . . . . . . . . 15

2.6.5 Negation Free Revision . . . . . . . . . . . . . . . . . . . . . 16

2.6 .6 Systems of Spheres . . . . . . . . . . . . . . . . . . . . 18

2.7 Non-Prioritized Revision _ . . . . . . . . . . . . . . . . . . . . . . . 19

2.7.1 Semi-revision . . . . . . . . . . . . . . . . . . . 19

2.7 .2 Selective Revision . . . . . . . . . . . . . . . . . . 20

2.8 The Nature of the Input . . . . . . . . . . . . . . . . . . . . . . 21

3 Prioritized Multiple Revision $\quad 23$

3.1 Early Steps on Multiple Operations _ . . . . . . . . . . . . . . . . 23

3.1.1 Multiple Contraction . . . . . . . . . . . . . . 23

3.1.1.1 Package Contraction . . . . . . . . . . . . 24 
3.1.1.2 Choice Contraction . . . . . . . . . . . . 24

3.1.2 Multiple Revision . . . . . . . . . . . . . . . . . 25

3.1.3 Generalizing Grove's Result . . . . . . . . . . . . . . . . . 26

3.1.4 Internal and External Revision for Belief Bases _ . . . . . . . 27

3.1.5 Multiple Package Partial Meet Revision . . . . . . . . . . . . . . . 30

3.1.5.1 Opening the Set . . . . . . . . . . . . 30

3.1.5.2 Completing the Construction .......... 31

3.1.5.3 Properties . . . . . . . . . . . . . . 31

3.1.5.4 Connecting with Contraction . . . . . . . . . 32

3.1.5.5 Matching Revision . . . . . . . . . . . . 32

3.1.5.6 Reduction . . . . . . . . . . . . . 33

3.2 Infinitary Belief Change . . . . . . . . . . . . . . . . . . 34

3.2 .1 General Contraction . . . . . . . . . . . . . . 34

3.2.2 Nice-Ordering Partition and Nice-Well-Ordering Partition . . . . 35

$3.2 .3 \quad$ New Properties . . . . . . . . . . . . . . . . . . . . . . . 36

3.2.4 Partial Meet Model . . . . . . . . . . . . . . . . . . . . 37

3.2.5 The Limit Postulate . . . . . . . . . . . . . . . . . . 37

3.2 .6 Constructing Revision . . . . . . . . . . . . . . . 38

3.2 .7 Connection with MaxNon's . . . . . . . . . . . . . . . 39

3.3 More on Systems of Spheres . . . . . . . . . . . . . . . . . . . 40

3.3.1 The Limit Assumption . . . . . . . . . . . . . . . . . . . 40

3.3.2 Well Behaved Functions _. . . . . . . . . . . . . . . . 40

3.3.3 Extra Constraints . . . . . . . . . . . . . . . . . . . 42

3.3.4 Topological Approach . . . . . . . . . . . . . . . . . . 43

3.4 Direct Constructions in Prioritized Revision . . . . . . . . . . . . . . . 44

3.4.1 Multiple Package Revision . . . . . . . . . . . . . . . . . . . . 44

3.4.2 Multi-Source Multiple Revision . . . . . . . . . . . . . . . . 46

3.4.2.1 Postulates and Construction . . . . . . . . . . 47

3.4.2.2 Comparing to Other Approaches . . . . . . . . . 49

3.5 Final Remarks . . . . . . . . . . . . . . . . . . . . . . . . . . . . . . . 49

3.6 Open Problems . . . . . . . . . . . . . . . . . . . 50

4 Non-Prioritized Multiple Revision $\quad \mathbf{5 3}$

4.1 The Levi Identity Model . . . . . . . . . . . . . . . . . . . . . . . . 53

4.1 .1 Internal Choice Revision . . . . . . . . . . . . . . 55

4.1.2 External Choice Revision . . . . . . . . . . . . 56

4.1.3 Common and Distinct Aspects . . . . . . . . . . . . . 57

4.1.4 Comparing with Selective Revision . . . . . . . . . . . . . 57 
4.2 The Descriptor Revision Approach . . . . . . . . . . . . . . . . . . . 57

$4.2 .1 \quad$ Descriptor Revision . . . . . . . . . . . . . . . . . . . 57

4.2.2 Choice Revision from Descriptor Revision . . . . . . . . . . . . 59

4.2 .3 Impossibility of Reduction . . . . . . . . . . . . . . . . 60

4.2.4 Making up One's Mind . . . . . . . . . . . . . . . . . . 60

4.3 The Multiple Believability Relations Approach . . . . . . . . . . . . . 61

4.3.1 Construction of Choice Revision . . . . . . . . . . . . . 62

4.4 The Semi-revision Approach . . . . . . . . . . . . . . . . . . . 62

4.4 .1 Explanations . . . . . . . . . . . . . . 62

4.4.2 A Revision Operator for Explanations . . . . . . . . . . . . 63

4.4.3 Belief Revision in Argumentative Systems . . . . . . . . . . . . . 64

4.5 Selective Revision and Deductive Argumentation . . . . . . . . . . . 65

4.6 Dealing with Inconsistent Inputs . . . . . . . . . . . . . . . . . 67

4.6.1 The Approach . . . . . . . . . . . . . . . . 68

4.6.2 Comparing with Iterated Revision _ . . . . . . . . . . . . . 69

4.7 The Core Beliefs Approach . . . . . . . . . . . . . . . . . . . . . . 70

4.7.1 Evaluative Multiple Revision . . . . . . . . . . . . 70

4.7.1.1 Evaluative Kernel Sets _. . . . . . . . . . . 72

4.7.1.2 Evaluative Remainder Sets . . . . . . . . . . . . 72

4.7.1.3 Comparing with Other Approaches . . . . . . . . . . 73

4.7.2 Rational Metabolic Revision . . . . . . . . . . . . . . . . . 74

4.7.2.1 Kernel Metabolic Revision . . . . . . . . . . . . 74

4.7.2.2 Partial Meet Metabolic Revision . . . . . . . . . . . 75

4.7.2.3 Comparing with Semi-revision . . . . . . . . . 75

$4.8 \quad$ Final Remarks . . . . . . . . . . . . . . . . . . . . . 76

4.9 Open Problems . . . . . . . . . . . . . . . . . . . . . . 76

5 Selective Base Revision $\quad 79$

5.1 Properties ............................ 80

5.2 Constructing the Operation . . . . . . . . . . . . . . . . . 81

5.3 Representation Results . . . . . . . . . . . . . . . . . . . . . 82

5.4 Negation Free Selective Base Revision . . . . . . . . . . . . . . . . . . . 83

$5.4 .1 \quad$ Properties . . . . . . . . . . . . . . . . 83

5.4 .2 Constructing the Operation . . . . . . . . . . . . 84

5.4.3 Representation Results . . . . . . . . . . . . . . . . 84

5.5 Related Work . . . . . . . . . . . . . . . . . . . . 85

6 Multiple Selective Revision $\quad 87$ 
6.1 Multiple Selective Theory Revision _. . . . . . . . . . . . . . . . 87

$6.1 .1 \quad$ Properties . . . . . . . . . . . . . . . . 88

6.1 .2 Constructing the Operation . . . . . . . . . . . . . 89

6.1.3 Representation Results . . . . . . . . . . . . . . . . . . . 91

6.2 Multiple Selective Base Revision . . . . . . . . . . . . . . . . . . . 92

$6.2 .1 \quad$ Properties . . . . . . . . . . . . . . . . 92

6.2 .2 Constructing the Operation . . . . . . . . . . . . . 93

6.2.3 Representation Results . . . . . . . . . . . . . . . . . . . 94

6.2 .4 Example . . . . . . . . . . . . . . . . . . 95

6.3 Related Work . . . . . . . . . . . . . . . . . . . . . . . 95

7 Multiple Choice Revision $\quad 97$

7.1 Properties . . . . . . . . . . . . . . . . . . . 97

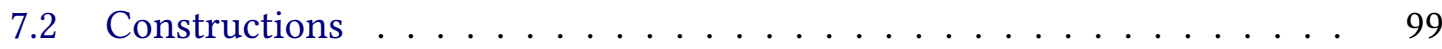

$7.2 .1 \quad$ Partial Meet . . . . . . . . . . . . . . . . . . . 99

7.2 .2 Kernel . . . . . . . . . . . . . . . . . . . 101

7.3 Maximal Expansion . . . . . . . . . . . . . . . . . . . . 103

7.4 Minimal Work . . . . . . . . . . . . . . . . . . . . . . . . . . . . 104

7.5 Example . . . . . . . . . . . . . . . . . . . . . . . . . 104

7.6 Connections between Selective Revision and Choice Revision ～. . . . . 105

7.6.1 From Selective to Choice . . . . . . . . . . . . . . . . . . . 105

7.6 .2 From Choice to Selective . . . . . . . . . . . . . . . . 106

7.7 Related Work . . . . . . . . . . . . . . . . . . . . . . . . . . . . . 107

8 Algorithms 109

8.1 Multiple Package Revision . . . . . . . . . . . . . . . . . . . . . . . 109

8.1.1 Package Partial Meet Revision . . . . . . . . . . . . . . . . . . 109

8.1.2 Package Kernel Revision . . . . . . . . . . . . . . . . . . . . 112

8.2 Multiple Choice Revision . . . . . . . . . . . . . . . . . . . . . . 115

8.2.1 Choice Partial Meet Revision _. . . . . . . . . . . . . . . 115

8.2.2 Choice Kernel Revision . . . . . . . . . . . . . . . . . . . . . . . 117

9 Conclusion $\quad 121$

9.1 Future Work . . . . . . . . . . . . . . . . . . . . . . . . . 122

\section{Appendices}


B Proofs related to Chapter 7

135

Bibliography

145 



\section{Chapter 1}

\section{Introduction}

Knowledge Representation is a field of Artificial Intelligence focused on defining models and resources to represent information in a way that a computer can use to solve complex tasks. However, according to Gärdenfors [Gär88], it is not very useful to know how to represent knowledge if at the same time we do not know how to change it when we are in the face of new information. Many systems work in a collaborative environment, which leads to a certain degree of information sharing. This sharing leads to an evolution of the agent's knowledge, which brings a drawback: the appearance of conflicts involving consistency. It is exactly this non-static aspect of knowledge that evinces the need of an agent to be able to deal with its dynamics. That is the context of the studies in the area of Belief Revision, which aims to handle the problem of adding or removing new information to/from a knowledge base in a consistent way. Or, in a simpler language, "belief revision is a choice that a cautious man makes in order to preserve consistency and retain as much information which he believes most as possible"[Zha96]. Most of the literature about Belief Revision is based on the AGM paradigm, a theory that became renowned after the seminal paper [AGM85] and whose name derives from the initials of its authors, Alchourrón, Gärdenfors and Makinson. The topics of this thesis are extensions or generalizations of this paradigm.

\subsection{Multiple Revision}

In the AGM paradigm, given a set of beliefs, there are three possible epistemic changes concerning a new belief: expansion, contraction and revision. Expansion occurs when the base simply absorbs the information without loss. A contraction consists in retracting beliefs from the base until the specified information is not derivable from it anymore. Finally, revision happens when the new belief is added consistently, possibly demanding a repair to eliminate inconsistency. In this thesis, we are going to focus on this last operation.

In the original framework, the new belief is assumed to be represented by a single formula. Nevertheless, there are situations in which the information by which we are going to revise a set of beliefs comes in a block, that is, a concurrent acceptance of many beliefs, maybe infinite. As we will see, in some situations, it may be possible to reduce multiple 
revision to singleton revision, for example, taking the conjunction of all new sentences, but it is not always feasible or even desirable. Thus, a framework for multiple changes is needed to cover these cases. The generalized operation proposed to fulfill this need is called Multiple Belief Revision (or Multiple Revision for short). It is different from belief merging, a kind of change operation in which preceding and new beliefs play symmetric roles. Merging is not going to be addressed here, and for more information, the reader is referred to [Fuh97, KP02a, FKIRS12]. Multiple Revision is also different from applying revision in an iterated or repeated way [Bou93, Nay94, DP97, KP00], taking the input set and revising sequentially, one by one. In Multiple Revision it is assumed that there is no preference over the input sentences, i.e., all of them have equal priority and should be processed simultaneously. Besides that, since the order in which you would process the sentences can make a difference in the final result, working with iterated revision may cause an asymmetry. In many approaches to iterated revision, if an agent revises by a sentence $\alpha$ and then by a sentence $\beta$ that is inconsistent with $\alpha$, then the result is the same as if only revising by $\beta$ [DJ12].

Thus, given the importance of the topic, this thesis starts with a survey on Multiple Revision, whose purpose is to summarize a literature review on the field since 1988, providing unified terminology and notation for readers and researchers that are interested in the subject or need an overview of the area. We also identify some limitations of the models and bring some comparisons between them.

One of AGM revision assumptions is that a new belief is always accepted, a property known as success. Revision operations of this kind are classified as prioritized revision. However, sometimes the agent should have the option to reject a piece of incoming information, either because of possible low reliability of the new belief (or of its source) or because of strong confidence in the previously held beliefs. That is why the field of nonprioritized revision started to be explored, in which the success property is not guaranteed. In this scenario, an agent receives a set of new beliefs and selects the most reliable ones to incorporate based on its previous knowledge.

Among the different varieties of non-prioritized revision ${ }^{1}$, Selective Revision [FH99] came up as a third possibility for the agent, since this operation allows it not only to accept or reject a new belief but also to accept just a part of it, that is, a weakening of the input sentence may be applied. This weakening is performed by a transformation function, to which the incoming information is submitted to perform an evaluation. Then the agent applies a traditional (prioritized) revision of its beliefs by the outcome of that function.

As well as AGM revision, Selective Revision was initially defined for single sentences as inputs. However, as explained above, a generalization to the multiple case would be useful. Barber and Kim [BK01], for example, state that in the real world, an agent is in contact with several information sources and deals with limited, incomplete, unsure or even wrong knowledge. Therefore, they developed a belief revision process ${ }^{2}$ which assesses the reputation of information sources and use it to define the next decision steps. Another

\footnotetext{
${ }^{1}$ For an overview see [FH18, Chapter 8].

${ }^{2}$ The belief revision process defined by them is a numerical formalism, unlike AGM, which is a logical formalism.
} 
example of the importance of multiple contexts is explored in [PSLS16] and [SPS18], in which the authors analyze the application of perception filters in agents. They consider simulation systems and robotic domains and observe that agents may be overwhelmed by unnecessary information without any goal control, thus generating a needless increase in processing time. The more sensors an agent has (to perceive an environment), the more perceptions it has to process, which becomes a bottleneck. Hence, pre-processing the information may decrease the cost effects of processing everything and advance an agent's performance.

Selective Revision was also initially defined for sets of sentences closed under logical consequence. Nevertheless, to make this approach closer to realistic scenarios, it would be interesting to extend its application to generic sets. Thus, this thesis proposes a generalization of Selective Revision to the multiple change context for both belief sets and belief bases. We provide constructions, postulates for the operators, properties for the transformation function and representation theorems to link everything for different classes of Multiple Selective Revision. It is essential to observe that, in a multiple-revision context, differently from the case of singleton inputs, the partial acceptance characteristic of selective revision can have two different meanings: either the simple choice of a subset of the input set or the logical weakening of a chosen subset (from the input). We are going to address both cases.

Another alternative for non-prioritized revision is Choice Revision. In a Multiple Revision context, there are two possible alternatives. If you want to incorporate the whole input set (a prioritized multiple revision), you need a Package Revision. On the other hand, if it is enough to absorb only a subset of it, then you need a Choice Revision. The difference between Multiple Selective Revision and Choice Revision lies on the way the beliefs are processed. In the selective approach, the agent first works with a transformation function in order to select what will be accepted from the input set (which will be followed by a package revision). In the choice approach, the agent changes its knowledge base in order to make it consistent with at least a subset of the input (which will be followed by a partial expansion by it). In addition, unlike Multiple Selective, in Choice Revision the beliefs accepted by an agent are restricted to what it receives, i.e., it cannot take just a logical weakening of some sentence.

Considering the requirement for choice (the acceptance of a subset of the input), one could think of a generalization of Credibility-limited revision [HFCF01, GFR18]. In that approach (proposed for a single-sentence input), there is a set of credible sentences, and an agent accepts to revise its beliefs only if the new belief is part of the credible sentences. In a generalized version, thus, there would be a previous selection on the input to decide the subset of it that would be considered, proceeding with a package revision on the chosen subset. However, this means that the decision would be made only based on of the input. In this thesis, we present more general constructions in which an agent can decide, looking at its previously held beliefs compared to the input set, what remains in its knowledge base, and what will be added. From [DG01, FKIRS12, $\mathrm{TDG}^{+} 19$ ] we can visualize practical scenarios for this kind of choice revision. Suppose you have a system in which a central agent receives information from multiple sources. The central agent has to process the new beliefs received in order to make some decisions. Suppose there is a credibility degree associated to each source, for example. The coordinator may consider such degree 
to decide which of its beliefs will be given up and which of the input sentences will be accepted. On the other hand, some agents may have the same (or similar) reliability rates, making the agent unsure about the final result of absorbing the information given by them. In this case, it would be interesting for the coordinator to process its knowledge in the face of the new beliefs, which will provide a kind of preview of the possible outcomes and facilitate the choice of a preferred final scenario. For this, it is necessary to delimit properties and constructions associated to them that will guide this process.

The first approaches for Multiple Revision were defined for propositional logic and sets of sentences closed under logical consequence. It makes the original operators not directly applicable in two important contexts: generic sets and logics without negation. When an operator requires logics closed under the negation of formulas, it cannot be directly applicable to many interesting logics, such as most Description Logics and Horn Logics. In this thesis, we propose two operators for Choice Revision of generic sets: one based on remainder sets (Partial Meet) and the other on kernel sets (Kernel), each characterized by a set of postulates, a construction and a representation theorem. Moreover, the proposed constructions do not depend on the negation of formulas. In the end, algorithms for the constructions are also proposed and proved to be correct. In addition, it is relevant to notice that, unlike most existing approaches for revision, the operators proposed here do not directly use contraction as an intermediate step.

\subsection{Publications}

This thesis includes portions of studies presented as papers in international workshops and conferences:

- Fillipe Resina and Renata Wassermann. A Survey on Multiple Revision. In Workshop Notes of the 18th International Workshop on Non-Monotonic Reasoning (NMR), 2020, pages 217-226. A short version of the survey that now is distributed between Chapters 2, 3 and 4.

- Fillipe Resina, Marco Garapa, Renata Wassermann, Eduardo Fermé, and Maurício Reis. Choosing What to Believe - New Results in Selective Revision. In Proceedings of the 17th International Conference on Principles of Knowledge Representation and Reasoning (KR 2020), volume 17, pages 687-691, 2020. It covers the extension of Selective Revision to belief bases and to logics without negation, which is now part of Chapter 6.

- Fillipe Resina and Renata Wassermann. Multiple Selective Revision. To be published in Workshop Notes of the 19th International Workshop on Non-Monotonic Reasoning (NMR 2021). Generalization of Selective Revision to the multiple case, which is now part of Chapter 6.

\subsection{Organization of the Thesis}

This thesis is organized as follows. Chapter 2 brings the beginnings of revision operation for singleton inputs and some of the variants that were proposed in the years that followed. 
Chapter 3 shows an overview of the different approaches proposed for Prioritized Multiple Revision. Chapter 4 does the same for the Non-Prioritized kind.

Chapter 5 presents an extension of Selective Revision to belief bases and logics without negation. Chapter 6 brings the generalization of Selective Revision to the multiple case. Chapter 7 focuses on the operation of Choice Multiple Revision and its axiomatic characterization. The Choice Revision algorithms are explored in Chapter 8.

Finally, Chapter 9 contains some conclusions, the summary of contributions and also some possible next steps to the topics of this thesis. The proofs of the main results presented in Chapters 6 and 7 were grouped in Appendices A and B, respectively.

The original contributions of this thesis begin in Chapter 5 . 



\section{Chapter 2}

\section{Beginnings of Revision}

This chapter summarizes different existing approaches for the revision operation when it is singleton on the right, i.e., when the input is a single piece of information. It brings most of the works that originated the operation, showing the many names given to it over time and the different properties and constructions proposed for it. A section is dedicated to exploring some approaches to non-prioritized revision, whose generalization to the multiple case is the focus of most of the presenting thesis.

Before starting to study revision, we provide information about the notation that will be used, epistemic states and epistemic attitudes.

\subsection{Notation}

Along with the models and operations studied in this thesis, we are going to assume a formal language $\mathcal{L}$ ruled by a logic identified by a consequence relation. Regarding logical consequence, we are going to use $C n$ to represent an operator that, receiving a set of logical sentences, returns the set of sentences that results logically from the input set.

For atomic formulas and sentences, we will use lowercase Greek letters $(\alpha, \beta, \ldots)$. For sets of sentences, uppercase Latin letters: $A, B, C, \ldots . K$ is reserved to represent a belief set $(K=C n(K))$. We are going to use $\perp$ for the falsity constant and $K_{\perp}$ for the inconsistent belief set. The default boolean connectives are considered: $\neg, \wedge, \vee, \rightarrow, \leftrightarrow$. $\wedge A$ stands for set conjunction and $\bigvee A$ stands for set disjunction. We denote the power set of $A$ by $\mathcal{P}(A)$.

A non-empty set is considered strongly inconsistent if every piece of it is inconsistent itself. A (partial) preorder relation $\leq$ on a nonempty set $A$ is a binary relation on $A$ which is reflexive and transitive. A preorder $\leq$ is called total if any two elements in $A$ are comparable, that is, for any $\alpha, \beta \in A$, we have either $\alpha \leq \beta$ or $\beta \leq \alpha$. 
2 | BEGINNINGS OF REVISION

\section{$2.2 \quad$ Epistemic States}

All of the works presented here involve epistemic states - elements that play a key role in an epistemological theory, which, in turn, focuses on exploring and understanding the changes in beliefs. The entities used to represent some agent's real or possible cognitive states in a given context are called epistemic states. Among various existing models, we point out two that will be explored in this thesis:

1. Sentential models: the representation is in the form of a set of sentences from some give language. The general idea is that each sentence speaks for one of the agent's beliefs. Usually, these sets are demanded to be consistent (given that a rational agent is not supposed to have conflicting beliefs), but this requisite may be relaxed in some contexts. Depending on the existence of an assumption about logical closure, we can have two different kinds of sets of sentences:

- Belief sets: sets of sentences closed under logical consequence, also known as theories. The purpose behind this approach is to consider that the respective agent is logically omniscient and, hence, shall believe in all the logical consequences of its beliefs. Due to the usually infinite nature of belief sets, they are more suitable for idealized agents and, consequently, challenging to handle computationally. In addition, it is impossible to differentiate explicit and derived knowledge.

- Belief bases: sets of sentences not necessarily closed under logical consequence, an approach closer to realistic scenarios and more suitable for computational purposes. In the works considered in this thesis, the chosen model for bases is the one proposed by Hansson[Han91, Han99b].

2. Possible Worlds Models: the representation is in the form of a set of possible worlds - a possible world is a consistent complete theory. The intended meaning of a set like this is that the associated agent knows that the "real world" is an element of that set, although it can be any of its elements.

\subsection{Epistemic Attitudes}

Before defining revision, it is important to understand the concept of epistemic attitude, which is a characterization of the status of the pieces of belief that are part of an epistemic state. In other words, an agent may consider a specific statement as certain, probable, possible, etc. However, the more epistemic attitudes are considered in a system, the more difficult it is to study them.

In sentential models, three possible epistemic attitudes in relation to a proposition $\alpha$ are acceptance, rejection and indetermination:

- $\alpha$ may be accepted - $\alpha$ is implied by the set of propositions

- $\alpha$ may be rejected - $\neg \alpha$ is implied by the set of propositions

- $\alpha$ is undetermined - neither $\alpha$ nor $\neg \alpha$ is implied by the set of propositions 
If an epistemic state is consistent, there is only one epistemic attitude regarding each of its elements. Nevertheless, knowledge is usually not static, which means that the set of beliefs of an agent may change. That is why we also have epistemic changes: alterations that happen in the epistemic states.

\subsection{The Revision Operation}

In some situations, an agent is forced to accept new information whose negation was previously accepted. So, the agent has to replace one or more of its sentences to avoid incompatibility. Besides, it is important that the change is not greater than needed, aiming at information economy. This kind of change is known as revision, although in past works of the area it was also called belief contravening change ${ }^{1}$ [Gär81], amendment ${ }^{2}$ [AM81, AM82, GR95], minimal change of belief [AM82, Gär84] and choice revision ${ }^{3}$ [AM82].

AGM revision is a function * that receives a set $K$ of beliefs, a new sentence $\alpha$ and returns a new set $K * \alpha$ in which $\alpha$ was consistently added.

In [Lev77], Isaac Levi referred to revision as a sequence of contractions (denoted by - ) and expansions (denoted by + ). An agent that wants to revise its beliefs by $\alpha$ should contract by $\neg \alpha$, add $\alpha$ and then close the resulting set under logical consequence. Inspired by this idea, Gärdenfors [Gär81] formally presented Levi's thesis as an identity, which was used in the AGM model to define revision. Let $K$ be the belief set of an agent and $\alpha$ an incoming belief. Considering the expansion operation defined as $K+\alpha=C n(K \cup \alpha)$, the Levi Identity defines the operation in the following way:

$$
K * \alpha=(K-\neg \alpha)+\alpha
$$

In the literature, this operation is also called internal revision [Han93]. Because of this view of revision as simply a compound operation, Isaac Levi claimed that contraction and expansion are the only legitimate forms of change in a corpus of knowledge. The Levi Identity, thus, becomes one of the forms to formally express the Decomposition Principle (DP)[Fuh89]: "Every legitimate revision is decomposable into a sequence of contractions and expansions." This principle is a presumption in the AGM framework, reducing the necessity for variety in belief change operations to solely two of them. However, as discussed in [Han17], if situations of "pure" contraction ${ }^{4}$ are usually unrealistic, contractions cannot

\footnotetext{
${ }^{1}$ Contravening refers to the fact that the new sentence violates the epistemic state of the agent.

${ }^{2}$ In deontic contexts (when referring to legal systems and legal codes), the process of amendment does not simply consist in including a new fragment of legislation; it is a substitution of one fragment by another that is incompatible with the former. So, it is composed by two steps: a removal and an inclusion.

${ }^{3}$ The name choice in the referred article is not related to the multiple context of revision but rather to the contraction function used to construct the revision operation. In Section 2.6.1 there is a more detailed explanation.

${ }^{4} \mathrm{~A}$ contraction is considered pure when no new belief is added to the corpus of knowledge, i.e., when it is performed for its own sake. Although it seems to be obvious from the definition of the operation, in real situations, it is not common to happen isolatedly. Cognitively, a contraction is usually motivated by the incoming of a new information that forces the agent to give up a previously held one. In most contractions the removal of an item is followed by the addition of its negation. For more discussions on when it is rational to contract, see [Lev83, $\mathrm{L}^{+}$91]
} 
be mandatory sub-operations of all revisions. In addition, Choice revision collides with DP since it is not clear how to directly reconstruct it using expansion and contraction. Besides, depending on the logic in question, it is not clear how to negate a formula, making it not viable to contract by negation [RW09], as demanded by the Levi Identity. Therefore, revision is treated here as a legitimate operation.

For further considerations about the relations between contractions and revisions, see [Gär88].

\subsection{Properties}

In order to establish the properties which the operations should obey, the AGM theory defines some rationality postulates. According to [AM82], Gärdenfors' minimal set of postulates for revision[Gär82] were four:

$$
\begin{aligned}
& \text { (success) } \alpha \in K * \alpha \\
& \text { (vacuity 1) If } \neg \alpha \notin C n(K) \text {, then } K * \alpha=K+\{\alpha\} \\
& \text { (vacuity 2) If } \alpha \in K \text { then } K * \alpha=K \\
& \text { (extensionality) If } C n(\alpha)=C n(\beta) \text {, then } K * \alpha=K * \beta
\end{aligned}
$$

Gärdenfors also suggested two other rules known as supplementary postulates, which the revision operation could also satisfy:

(conjunctive inclusion) $K *(\alpha \wedge \beta) \subseteq C n((K * \alpha) \cup\{\beta\})$

(conjunctive vacuity) $C n((K * \alpha) \cup\{\beta\}) \subseteq K *(\alpha \wedge \beta)$ for any theory $K$, provided that $\neg \beta \notin K * \alpha$

In addition, it has been noted by Gärdenfors[Gär81] that, in the spirit of the Levi Identity, it is also possible to define contraction from revision via the following rule: $K-\alpha=K \cap(K * \neg \alpha)$. In [AM82] it was called the Gärdenfors Identity and from [Gär88] on it has been called the Harper Identity ${ }^{5}$

In [AGM85], the authors summarized the postulates for revision as done in [Mak85]. Makinson started bringing the closure and success postulates as direct consequences of the definition of revision via the Levi Identity. He recovers from Gärdenfors the vacuity 1 and extensionality postulates and adds to the list the consistency property and the Harper Identity. Finally, he refers to the supplementary postulates, forming a set of 8 properties. So, comparing to the original list, the new ones are:

(closure) $K * \alpha$ is a belief set

(weak consistency) If $\neg \alpha \notin C n(\varnothing)$, then $K * \alpha$ is consistent under $C n$

(Harper Identity) $(K * \alpha) \cap K=K-\neg \alpha$ whenever $K$ is a belief set

\footnotetext{
${ }^{5}$ Gärdenfors possibly decided to not accept his name for the identity due to the fact that the inspiration for it came from the ideas expressed in a previous work of William Harper [Har76].
} 
Although this last property appeared in the original AGM definition[AGM85], it became unusual to refer to it in the set of revision postulates. Makinson observed in [Mak87] that, differently from the other postulates, the Harper Identity involves both of the operations (revision and contraction) and, instead of being considered one of the properties, it should be seen as a way to obtain contraction from revision, as was the original intention.

In [Gär88], Gärdenfors took this proposal forward, removing the referred identity from the list of postulates and adding the postulate of inclusion:

(inclusion) $K * \alpha \subseteq K+\{\alpha\}$

Vacuity 2 was shown as a consequence of the other postulates for revision and the ones for expansion, remaining out of the list. This way, he grouped the revision postulates as they are traditionally known:

$\left(\mathbf{K}^{*} \mathbf{1}\right) K * \alpha$ is a belief set

$\left(\mathbf{K}^{*} 2\right) \alpha \in K * \alpha$

$\left(\mathbf{K}^{*} 3\right) K * \alpha \subseteq K+\{\alpha\}$

$\left(\mathbf{K}^{*} \mathbf{4}\right)$ If $\neg \alpha \notin C n(K)$, then $K * \alpha=C n(K \cup\{\alpha\})$

(K $\mathbf{K}^{*} \mathbf{5}$ If $\neg \alpha \notin C n(\varnothing)$, then $K * \alpha$ is consistent under $C n$

(K*) If $C n(\alpha)=C n(\beta)$, then $K * \alpha=K * \beta$

$\left(\mathbf{K}^{*} 7\right) K *(\alpha \wedge \beta) \subseteq \operatorname{Cn}((K * \alpha) \cup\{\beta\})$

$\left(\mathbf{K}^{*} \mathbf{8}\right) C n((K * \alpha) \cup\{\beta\}) \subseteq K *(\alpha \wedge \beta)$, provided that $\neg \beta \notin K * \alpha$

\subsection{Constructions}

In this section, we are going to present some constructive modellings, i.e., functions that have the role to show how an agent may construct a specific operation.

Since in the AGM model the definition of revision is given through contraction, we are going to start with a construction for this last one.

\subsubsection{Partial Meet Contraction}

Partial Meet contraction was first suggested in [AM82], being explored with more details in [AGM85] and, later, being generalized for belief bases, as can be seen in [Han99b]. In order to define it, we need to have some tools in hand.

This construction is based on maximal non-implying subsets, as can be seen in the following definition:

Definition 1. [AM81] Let $K$ be a set of sentences and $\alpha$ a sentence. The set $K \perp \alpha$ (remainder set of $K$ in relation to $\alpha$ ) is the set such that $X \in K \perp \alpha$ iff:

- $X \subseteq K$

- $X \forall \alpha$ 
- if exists $X^{\prime}$ such that $X \subset X^{\prime} \subseteq K$, then $X^{\prime} \vdash \alpha$

So, the elements of the remainder set $K \perp \alpha$ are the maximal subsets of $K$ that do not imply $\alpha$. The idea is to select some of these elements and intersect them ${ }^{6}$. For that purpose, we need a special function:

Definition 2. [AGM85] Let $K$ be a set of sentences. A selection function for $K$ is a function $\gamma$ such that for all sentences $\alpha$ :

- if $K \perp \alpha \neq \varnothing$, then $\gamma(K \perp \alpha) \neq \varnothing$ and $\gamma(K \perp \alpha) \subseteq K \perp \alpha$

- if $K \perp \alpha=\varnothing$, then $\gamma(K \perp \alpha)=\{K\}$

In [AM82], the selection function was called choice function and the contraction based on a choice function was named choice contraction, while the revision function based on a choice contraction (via the Levi Identity) was called choice revision.

Finally, having the remainder set and a selection function, it is possible to define the operation:

Definition 3. [AGM85] Let $K$ be a set of sentences and $\gamma$ a selection function for $K$. The partial meet theory contraction on $K$ that is generated by $\gamma$ is the operation ${ }_{\gamma}$ such that for all sentences $\alpha$ :

$$
K-_{\gamma} \alpha=\bigcap \gamma(K \perp \alpha)
$$

With the completion of this construction, it is possible to proceed to the revision counterpart.

\subsubsection{Partial Meet Revision}

From the AGM framework, we have the partial meet contraction applied to the Levi Identity in order to define the corresponding construction for (internal) revision:

Definition 4. [AGM85] Let $\gamma$ be a selection function for the belief set $K$. Then the operator ${ }^{*} \gamma$ of (internal) partial meet theory revision for $K$ is defined as follows:

$$
K *_{\gamma} \alpha=\left(K-{ }_{\gamma} \neg \alpha\right)+\alpha
$$

It is clear that this revision depends on the selection function and the consequence operator. AGM framework departs from some premises about the underlying logic $\langle\mathcal{L}, C n\rangle$ (the language and the corresponding consequence operator). The logic is supposed to be tarskian $^{7}$ supra-classical $^{8}$ and closed under the standard connectives $(\wedge, \vee, \rightarrow$ and $\neg)$. The consequence operator is supposed to be compact ${ }^{9}$, and satisfy the rule of introduction of

\footnotetext{
${ }^{6}$ For a discussion on how to choose the "most important" elements, see [Gär84].

${ }^{7} \mathrm{~A}$ logic is tarskian if its associated consequence operator satisfies, for all $A, B \in \mathcal{P}(\mathcal{L})$ : monotonicity (if $A \subseteq B$, then $C n(A) \subseteq C n(B))$, inclusion $(A \subseteq C n(A))$ and idempotence $(C n(A)=C n(C n(A)))$.

${ }^{8} \mathrm{~A}$ logic is supra-classical if it contains every valid inference of Classical Propositional Logic.

${ }^{9}$ A consequence operator is compact if, given $A \in \mathcal{P}(\mathcal{L})$, for every $\alpha \in A$ there is a finite subset $A^{\prime} \subseteq A$ such that $\alpha \in C n\left(A^{\prime}\right)$.
} 
disjunctions in the premises ${ }^{10}$ and the deduction theorem ${ }^{11}$. This set of properties regarding the logic and its consequence operator is known as the AGM assumptions.

Observation 1. [AGM85] Let $K$ be a belief set. An operator * on $K$ is a partial meet (theory) revision function iff it satisfies $(K * 1)-(K * 6)$. If, in addition, it satisfies the supplementary postulates, it is a transitively relational partial meet revision.

Although the seminal paper by Alchourrón et al. did not ignore open belief sets, all of the main results demand closure of the belief set. However, it is important to know how to deal with belief bases.

\subsubsection{Working with Belief Bases}

For belief bases, as can be seen in [Han93], the definition of internal partial meet revision can be directly translated from belief sets, except for the closing of the set under logical consequence. Let $B$ be a belief base. Then:

$$
B * \alpha=(B-\neg \alpha) \cup\{\alpha\}
$$

The following postulates were proposed for belief base revision:

(success) $\alpha \in B * \alpha$

(inclusion) $B * \alpha \subseteq B \cup\{\alpha\}$

(weak consistency) if $\alpha H \perp$ then $B * \alpha H \perp$

(non-contradiction) If $\alpha H \perp$, then $B * \alpha H \neg \alpha$

(uniformity) If for all $B^{\prime} \subseteq B, B^{\prime} \cup \alpha \vdash \perp$ is inconsistent iff $B^{\prime} \cup \beta \vdash \perp$, then $B \cap(B * \alpha)=B \cap(B * \beta)$

(vacuity) If $\neg \alpha \notin C n(B)$, then $B * \alpha=B \cup\{\alpha\}$

(relevance) If $\beta \in B$ and $\beta \notin B * \alpha$, then there is some $B^{\prime}$ such that $B * \alpha \subseteq B^{\prime} \subseteq$ $B \cup\{\alpha\}, B^{\prime}+\perp$ but $B^{\prime} \cup\{\beta\} \vdash \perp$

The next observation shows that vacuity can be obtained as a consequence of other postulates:

Observation 2. [Han99b] If an operator * for B satisfies success, inclusion and relevance, then it satisfies vacuity.

The definition of base revision by means of a partial meet construction is given as follows:

Definition 5. [Han93, Han99b] Let B be a belief base. The partial meet base revision on $B$ based on a selection function $\gamma$ is the operator ${ }_{\gamma}$ such that for all sentences $\alpha$ :

$$
B *_{\gamma} \alpha=\left(B-{ }_{\gamma} \alpha\right) \cup\{\alpha\}
$$

\footnotetext{
${ }^{10} \alpha \in C n(A \cup\{\beta \vee \gamma\})$ whenever $\alpha \in C n(A \cup\{\beta\})$ and $\alpha \in C n(A \cup\{\gamma\})$.

${ }^{11} \beta \in C n(A \cup\{\alpha\})$ iff $\alpha \rightarrow \beta \in C n(A)$.
} 
The next theorem is a characterization of partial meet revision on belief bases:

Theorem 1. [Han99b] The operator * is an operator of partial meet theory revision for a belief base B iff it satisfies success, inclusion, consistency, relevance and uniformity.

When dealing with belief bases, it is possible to recover from inconsistencies, as we do not close the set under logical consequences. Hansson [Han93] has proposed an alternative construction for revision based on the idea of reversing the Levi identity, i.e., reversing the order in which contraction and expansion are performed. This alternative approach was called external revision. Before defining it, we need to have another kind of selection function. The function shown in Definition 2 is a one-place selection function, i.e., it takes only one argument.

Definition 6. [Han93] A two-place selection function is a function $\gamma$ such that for each subset $K$ of $L, \gamma(K)=,\gamma_{K}()$ is a one-place selection function for $K$. Each two-place selection function gives rise to an operator of global partial meet contraction $-_{\gamma}$ such that for all sets $K$ and sentences $\alpha: K{ }_{\gamma} \alpha=\bigcap \gamma_{K}(K \perp \alpha)$.

Now, it is possible to define external revision:

Definition 7. [Han99b] Let $\gamma$ be a two-place selection function for the belief base B. Then the global operator $\pm_{\gamma}$ of external partial meet base revision is defined as follows. For all sentences $\alpha$ :

$$
B \pm_{\gamma} \alpha=(B \cup\{\alpha\})-{ }_{\gamma} \neg \alpha
$$

This corresponds to first adding the formula $\alpha$ and then contracting the result by $\neg \alpha$. In order do characterize this operation, we need two extra postulates:

(pre-expansion) $(B \cup\{\alpha\}) * \alpha=B * \alpha$

(weak uniformity) If $\alpha$ and $\beta$ are elements of $B$ and it holds for all $B^{\prime} \subseteq B$ that $\neg \alpha \in C n\left(B^{\prime}\right)$ iff $\neg \beta \in C n\left(B^{\prime}\right)$, then $B \cap(B * \alpha)=B \cap(B * \beta)$

Theorem 2. [Han93] An operator * is an operator of external partial meet base revision on $B$ iff it satisfies non-contradiction, inclusion, success, relevance, pre-expansion and weak uniformity.

As can be observed, external revision does not work for belief sets. If $\alpha$ is inconsistent with $K$, the expansion of $K$ by $\alpha$ trivializes the set and ruins the revision process. Therefore, this kind of operation was thought for belief bases. In [Han99b] the author gave some examples to show different situations when one operation or the other is closer to the actual psychological process. In addition, the following result shows that usually internal and external revision do not coincide:

Observation 3. [Han93]

1. Uniformity does not hold in general for external partial meet revision.

2. Pre-expansion does not hold in general for internal partial meet revision. 


\subsubsection{Kernel Contraction}

In [Han94], Hansson introduced another construction for contraction operators, called kernel contraction, which is a generalization of safe contraction[AM85]. It is based on the concept of minimal implying subset, named kernel set $(\Perp)$ :

Definition 8. [Han94] $X \in B \Perp \alpha$ (the kernel set of $B$ in relation to $\alpha$ ) iff:

1. $X \subseteq B$

2. $\alpha \in C n(X)$

3. for all $Y$, if $Y \subset X$ then $\alpha \notin C n(Y)$

The elements of $B \Perp \alpha$ are called $\alpha$-kernels.

Now, we need to remove at least one element from each of the $\alpha$-kernels by means of an incision function:

Definition 9. [Han94] An incision function for B is any function $\sigma$ such that for any formula $\alpha$ :

1. $\sigma(B \Perp \alpha) \subseteq \bigcup(B \Perp \alpha)$, and

2. If $\varnothing \neq X \in B \Perp \alpha$, then $X \cap \sigma(B \Perp \alpha) \neq \varnothing$.

Then, it is possible to define a kernel contraction:

Definition 10. [Han94] Let $\sigma$ be an incision function. The kernel base contraction on $B$ determined by $\sigma$ is the operation ${ }_{\sigma}$ such that for all sentences $\alpha$ :

$$
B-{ }_{\sigma} \alpha=B \backslash \sigma(B \Perp \alpha)
$$

\subsubsection{Kernel Revision}

Analogously to what happens with partial meet, from the definition of kernel contraction, one can obtain a kernel revision:

Definition 11. [Han99b, HW02] The (internal) kernel base revision on $B$ based on an incision function $\sigma$ is the operator ${ }_{\sigma} \sigma$ such that for all sentences $\alpha: B{ }_{\sigma} \alpha=\left(B-{ }_{\sigma} \alpha\right) \cup\{\alpha\}$

In order to characterize this operation, we need the following extra postulate:

(core-retainment) If $\beta \in B$ and $\beta \notin B * \alpha$, then there is some $B^{\prime}$ such that $B^{\prime} \subseteq B \cup\{\alpha\}$, $B^{\prime} \forall \perp$ but $B^{\prime} \cup\{\beta\} \vdash \perp$.

Theorem 3. [Han99b, HW02] An operator * is an operator of internal kernel base revision iff it satisfies non-contradiction ${ }^{12}$, inclusion, core-retainment, success and uniformity.

In an operation of external kernel revision by $\alpha$, the belief base is first expanded with $\alpha$ and then kernel contraction by $\neg \alpha$ takes place:

Definition 12. [Han99b, HW02] The external kernel base revision of B based on an incision function $\sigma$ is the operator ${ }_{\sigma}$ such that for all sentences $\alpha$ :

\footnotetext{
${ }^{12}$ The postulate of non-contradiction could be replaced by weak consistency
} 


$$
B *_{\sigma} \alpha=(B \cup\{\alpha\}) \backslash \sigma((B \cup\{\alpha\}) \Perp \neg \alpha)
$$

The following theorem characterizes this operation:

Theorem 4. [Han99b, HW02] An operator * is an operator of external kernel base revision iff it satisfies non-contradiction ${ }^{12}$, inclusion, success, core-retainment, pre-expansion and weak uniformity.

\subsubsection{Negation Free Revision}

Although in [Han99b, HW02] there are definitions and constructions for partial meet and kernel base revisions, they use contraction as an intermediate step (Levi Identity), which makes the respective representation theorems dependent on the negation of sentences. In addition, as studied in [FPA04, FPA05a, FPA05b], due to the AGM-assumptions, the direct application of the AGM-paradigm is impossible in some interesting logics, such as many Description Logics[BCM ${ }^{+}$03, BHLS17] and also Horn Logic.

Aiming at solving this issue, Ribeiro and Wassermann [RW09] proposed and axiomatically characterized some base revision operators without assuming that the underlying logic is closed under negation of sentences. They worked only with external revision, i.e., all the constructions adopted the same method: firstly, the expansion of the belief base by the input $\alpha$ and, then, a (partial meet or kernel) contraction of the resulting base by $\perp$. Within this approach, the dependence on negation is warded off by establishing conditions on the selection or incision functions.

Definition 13. [RW09] A selection function $\gamma$ that protects the input is defined as:

1. If $\alpha H \perp$, then $\varnothing \neq \gamma((B+\alpha) \perp \perp, \alpha) \subseteq(B+\alpha) \perp \perp$ and $\alpha \in \bigcap \gamma((B+\alpha) \perp \perp, \alpha)$.

2. $\gamma((B+\alpha) \perp \perp, \alpha)=\{B\}$ otherwise

Note that this definition of selection function takes two arguments. The second one is the formula to be preserved. This is used so that the function picks only those remainders which contain $\alpha$, which means that the usage of this kind of selection function implies that success is a strong requirement for the operation, while consistency is weak (as the input will be absorbed anyway). The same happens with the incision function defined after the theorem below. In [RW09] the names of the operators constructed this way end with the indication with success.

Theorem 5. [RW09] The operator * is a negation free external partial meet base revision for a belief base B iff it satisfies success, inclusion, weak consistency, relevance and pre-expansion.

The same idea is used for incision functions:

Definition 14. [RW09] An incision function that protects the input is defined as a function $\sigma$ that satisfies:

1. $\sigma(\alpha, B \Perp \perp) \subseteq \bigcup(B \Perp \perp)$

2. If $\alpha \forall \perp$ and $\varnothing \neq X \in B \Perp \perp$, then $X \cap \sigma(\alpha, B \Perp \perp) \neq \varnothing$

3. $\alpha \notin \sigma(\alpha, B \Perp \perp)$ 
Theorem 6. [RW09] The operator * is a negation free external kernel base revision for a belief base B iff it satisfies success, inclusion, weak consistency, core-retainment and pre-expansion.

For the negation free (internal) revisions of belief bases, one can easily adapt a definition originally proposed in the context of belief sets. A negation free remainder set $B \downarrow \alpha$ is defined as the set of all maximal subsets of $B$ that are consistent with the input $\alpha .{ }^{13}$ Formally:

Definition 15. [RW14][negation free remainder set]

$X \in B \downarrow \alpha$ iff:

1. $X \subseteq B$

2. $X \cup\{\alpha\} H \perp$

3. If $X \subset X^{\prime} \subseteq B$ then $X^{\prime} \cup\{\alpha\} \vdash \perp$

A selection function $\gamma$ selects a non-empty subset of $B \downarrow \alpha$ if possible i.e. if $B \downarrow \alpha \neq \varnothing$. Otherwise it returns $\{B\}$. Any selection function induces the following revision operation called negation free internal partial meet revision:

$$
B *_{\gamma} \alpha=\bigcap \gamma(B \downarrow \alpha) \cup\{\alpha\}
$$

Then, we propose the following representation theorem:

Theorem 7. An operator $*$ is an operator of negation free internal partial meet base revision iff it satisfies weak consistency, inclusion, success, relevance and uniformity.

The proof can be easily adapted from [HW02] by using $B \downarrow \alpha$ instead of $B \perp \neg \alpha$.

The same strategy can be used for kernel revision:

Definition 16. [RW14][negation free kernel]

$X \in B \downarrow \alpha$ iff:

1. $X \subseteq B$.

2. $X \cup\{\alpha\} \vdash \perp$.

3. If $X^{\prime} \subset X$ then $X^{\prime} \cup\{\alpha\} H \perp$.

Then, it is possible to define the following revision operation called negation free internal kernel revision:

$$
B *_{\sigma} \alpha=B \backslash \sigma(B \downarrow \alpha) \cup\{\alpha\}
$$

Now, the respective representation result:

Theorem 8. An operator $*$ is an operator of negation free internal kernel base revision iff it satisfies weak consistency, inclusion, success, core-retainment and uniformity.

\footnotetext{
${ }^{13}$ This was called an inconsistency-based remainder set in [Del08].
} 
Once more, we omit the proof, as it can be easily adapted from [HW02], using $B \downarrow \downarrow \alpha$ instead of $B \Perp \neg \alpha$.

\subsubsection{Systems of Spheres}

In [Gro88], Grove introduced a new kind of construction, based on possible worlds.

A possible world (a consistent complete theory) is a maximal consistent subset of $\mathcal{L}$. The set of all possible worlds of $\mathcal{L}$ will be represented by $\mathcal{M}_{\mathcal{L}}$ and, for each $U \subseteq \mathcal{M}_{\mathcal{L}}$, the complement set of $U$ will be denoted by $\bar{U}$.

Definition 17. [Gro88] Let $A$ be a set of sentences of $\mathcal{L}$. The set of possible worlds that contain $A$ is denoted by $[A]$, i.e., $[A]=\left\{M \in \mathcal{M}_{L}: A \subseteq M\right\}$. If $A$ is inconsistent this will be the empty set. The elements of $[A]$ are the $A$-worlds. For a sentence $\varphi \in \mathcal{L},[\varphi]$ is an abbreviation of $[\{\varphi\}]$. The elements of $[\varphi]$ are the $\varphi$-worlds.

A set $\mathcal{V}$ of consistent complete theories is elementary iff $\mathcal{V}=[\cap \mathcal{V}]$. In words, $\mathcal{V}$ is elementary iff the possible worlds satisfying $\cap \mathcal{V}$ are precisely those contained in $\mathcal{V}$ (no world outside $\mathcal{V}$ is compatible with the theory $\cap \mathcal{V}$ ).

Now, it is possible to define the construction:

Definition 18. [Gro88] Let $\mathcal{X}$ be a subset of $\mathcal{M}_{\mathcal{L}}$. A system of spheres centered on $\mathcal{X}$ is a collection $\mathrm{S}$ of subsets of $\mathcal{M}_{\mathcal{L}}$, i.e., $\mathrm{S} \subseteq \mathcal{P}\left(\mathcal{M}_{\mathcal{L}}\right)$, that satisfies the following conditions:

(S1) $\mathrm{S}$ is totally ordered with respect to set inclusion; that is, if $\mathcal{V}, \mathcal{V} \in \mathrm{S}$, then $\mathcal{V} \subseteq \mathcal{V}$ or $\mathcal{V} \subseteq \mathcal{V}$.

(S2) $\mathcal{X} \in \mathrm{S}$, and if $\mathcal{U} \in \mathrm{S}$ then $\mathcal{X} \subseteq \mathcal{V}$.

(S3) $\mathcal{M}_{\mathcal{L}} \in \mathrm{S}$ (and so it is the largest element of S).

(S4) For every $\varphi \in \mathcal{L}$, if there is any element in $\mathrm{S}$ intersecting $[\varphi]$ then there is also a smallest element in $\mathrm{S}$ intersecting $[\varphi]$.

The elements of S are called spheres.

For any consistent sentence $\varphi \in \mathcal{L}$, the smallest sphere in $\mathrm{S}$ intersecting $[\varphi]$ is denoted by $C_{\mathrm{S}}(\varphi) . f_{\mathrm{S}}(\varphi)$ denotes the set consisting of the $\varphi$-worlds closest to $\mathcal{X}$, i.e., $f_{\mathrm{S}}(\varphi)=[\varphi] \cap C_{\mathrm{S}}(\varphi)$.

Essentially, a system of spheres is a total preorder on possible worlds satisfying a specific smoothness condition.

A sphere $V \in \mathrm{S}$ is said to be proper if it contains at least one world outside all spheres smaller than $\mathcal{V}$. The core of $\mathcal{V}$, denoted by $\mathcal{V}^{c}$, is the set $\mathcal{V}^{c}=\bigcup\{V \in \mathrm{S}: V \subseteq \mathcal{V}$. So, a sphere $V \in \mathrm{S}$ is proper iff $\mathcal{V} \neq V^{c}$.

Grove also established a connection between systems of spheres and AGM revision:

Definition 19. [Gro88] Consider a theory $K$ of $\mathcal{L}$ and let $\mathrm{S}$ be a system of spheres centered on $[K]$. The S-based revision on $K$ is the revision operation *s defined, for any $\varphi \in \mathcal{L}$, by: 


$$
K *_{\mathrm{S}} \varphi= \begin{cases}\cap f_{\mathrm{S}}(\varphi) & \text { if }[\varphi] \neq \varnothing \\ \mathcal{L} & \text { otherwise }\end{cases}
$$

Grove demonstrated that the class of functions obtained from systems of spheres by means of *s is exactly the family of AGM revision functions.

\subsection{Non-Prioritized Revision}

Non-prioritized revision is a class of revision operations whose main difference in relation to the traditional AGM revision is that the input information is not always accepted, i.e., success is not a desirable property. The point for it is that, contrary to what happens in prioritized revision, a new belief should not always have supremacy over previous beliefs. Although in some situations it is reasonable to consider new information as the most trustworthy and correct one, in a more realistic scenario it is not recommended to have it as a general assumption, which means that a new belief can be rejected if it conflicts with more entrenched or more valuable earlier beliefs.

\subsubsection{Semi-revision}

In [Han97] Hansson proposed an operation of revision that can be constructed based on another operation called consolidation[Han91]. A belief base is consolidated when a consistent subset of it is extracted, which essentially means to contract the base by a contradictory sentence:

Definition 20. [Han91] Let - be an operation of (global) contraction on belief bases. Then the operation!, such that for all belief bases B:

$$
B !=B-\perp
$$

is the consolidation based on -. If - is a partial meet contraction, then! is a partial meet consolidation. If - is a kernel contraction, then! is a kernel consolidation.

Having this operation, the semi-revision of a belief base $B$ by $\alpha$ is defined as the expansion of $B$ by $\alpha$ followed by the consolidation of $B$ :

Definition 21. [Han97] Let! be an operation of consolidation. The operation?, such that for all $B$ and $\alpha$ :

$$
B ? \alpha=(B \cup\{\alpha\}) !
$$

is the corresponding semi-revision operation. If! is a partial meet (kernel) consolidation, then the operation? defined as above is an operation of partial meet (kernel) semi-revision.

It inserts semi-revision in the expansion + consolidation variety of non-prioritized belief revision[Han99a]. The expansion + consolidation strategy has been established only for belief bases, for the same reason as exposed in Section 2.6.2.1 about external revision. 
For possibilities of interdefinability between semi-revision and consolidation, see [Han97].

\subsubsection{Selective Revision}

In [FH99], Fermé and Hansson explored a new possibility of revision which is capable of incorporating only a part of the input belief, calling it selective revision. Many models were developed to work with two options: complete acceptance of the input information or full rejection of it. So the selective revision model aimed to be a third possibility.

From the six basic AGM postulates, four are equally valid for selective revision: closure, inclusion, weak consistency and extensionality. The vacuity property is not always desirable in a selective revision framework, given that even if the new information is consistent with the initial beliefs, the agent may still want to select a part of it. Despite that, vacuity appeared in the original results and will also be used in ours.

One of the basic AGM postulates for revision is success, to ensure that the new belief will be accepted $(K * \alpha \vdash \alpha)$. Evidently, this property should not hold for selective revision (represented by $\circ$ ). There is a way to weaken it that it is to incorporate some part (a proxy) of the incoming belief (there is a sentence $\beta$, such that $K \circ \alpha \vdash \beta, \vdash \alpha \rightarrow \beta$ and $K \circ \alpha=K \circ \beta$ ). This proxy success postulate has a weaker version that consists in not requiring that $\vdash \alpha \rightarrow \beta$. Lastly, the consistent expansion postulate intends to establish that previous beliefs are discarded only to guarantee consistency (if $K \nsubseteq K \circ \alpha$ then $K \cup(K \circ \alpha) \vdash \perp)$. As stated in [FH99], consistent expansion follows from success and vacuity. For more details about the postulates for this operation, see [FH99].

In order to achieve the partial acceptance, we need to apply a filter called transformation function $f$ (defined from $\mathcal{L}$ to $\mathcal{L}$ ) to any input sentence $\alpha$ aiming to extract, roughly speaking, the most trustworthy part of it. A natural restriction is that $f(\alpha)$ should not contain more information that the one that is contained in $\alpha$ (i.e., $\vdash \alpha \rightarrow f(\alpha)$ ). However, the authors proposed the operation in a very general way such that this restriction is not necessarily required. The following were some of the proposed properties for transformation functions presented in [FH99]:

$$
\text { (implication) } \vdash \alpha \rightarrow f(\alpha)
$$

(weak implication) If $K \Vdash \neg \alpha$, then $\vdash \alpha \rightarrow f(\alpha)$

(idempotence) $\vdash f(f(\alpha)) \leftrightarrow f(\alpha)$

(extensionality) If $\vdash \alpha \leftrightarrow \beta$, then $\vdash f(\alpha) \leftrightarrow f(\beta)$

(consistency preservation) If $\forall \neg \alpha$, then $\forall \neg f(\alpha)$

(weak maximality) If $K \forall \neg \alpha$, then $\vdash f(\alpha) \leftrightarrow \alpha$

After applying the transformation function, the previous beliefs are revised by the result of the filtering in a prioritized way. With these tools in mind, it is possible to construct a model for selective revision:

Definition 22. [FH99] Let $K$ be a belief set, * ${ }_{\gamma}$ be a partial meet theory revision for $K$, and $f$ be a transformation function. The selective revision $\circ$, based on $*_{\gamma}$ and $f$, is the operation such 
that for all sentences $\alpha$ :

$$
K \circ \alpha=K{ }_{\gamma} f(\alpha)
$$

It inserts selective revision in the decision + revision variety of non-prioritized belief revision[Han99a].

We finish this section by recalling from [FH99] representation theorems for two classes of selective revision functions:

Theorem 9 ([FH99]). Let $K$ be a belief set, and $\circ$ be an operator on $K$. Then the following conditions are equivalent:

1. - satisfies closure, inclusion, vacuity, weak consistency, consistent expansion, extensionality and weak proxy success.

2. There exists a partial meet theory revision operator $*_{\gamma}$ for $K$ and a transformation function $f$ that satisfies extensionality, consistency preservation, weak maximality and idempotence such that $K \circ \alpha=K{ }_{\gamma} f(\alpha)$, for all $\alpha$.

Theorem 10 ([FH99]). Let $K$ be a belief set, and $\circ$ be an operator on $K$. Then the following conditions are equivalent:

1. - satisfies closure, inclusion, vacuity, weak consistency, consistent expansion, extensionality and proxy success.

2. There exists a partial meet theory revision operator $*_{\gamma}$ for $K$ and a transformation function $f$ that satisfies extensionality, consistency preservation, weak maximality, idempotence and implication such that $K \circ \alpha=K{ }_{\gamma} f(\alpha)$, for all $\alpha$.

It is important to observe that this approach for selective revision is not suitable neither for belief bases (given the required closure property) nor for non-classical logics (once some properties of the transformation function apply, for example, negation, disjunction and conjunction of sentences, which are not always defined for some logics).

\subsection{The Nature of the Input}

As shown in this chapter, the AGM framework supposes that the operations are always singular on the right. However, there are some situations when you have multiple sentences as input instead of a single sentence and need to perform a simultaneous revision by them. It demands a generalization of the theory in order to cover this kind of situation. Nevertheless, as observed by Peppas [Pep08], taking AGM to the multiple case is not as elementary as it may first seem, especially when dealing with infinite sets. Still, depending on the conditions of the underlying logic, there may be some challenges. In the next two chapters, we will show the different approaches proposed since 1988 to solve this problem. 



\section{Chapter 3}

\section{Prioritized Multiple Revision}

This chapter summarizes the main works existing in the literature regarding Prioritized Multiple Revision, i.e., when the input is totally incorporated in the final result. Section 3.1 brings the first approaches proposed for belief sets, belief bases and systems of spheres. After that, section 3.2 considers the cases when the input is allowed to be infinite, section 3.3 looks at further proposals for systems of spheres and section 3.4 shows the approaches that do not directly use contraction as an intermediate step.

As will be used, the generalization of expansion is very straightforward: if $K$ and $A$ are sets of sentences, $K+A=C n(K \cup A)$.

\subsection{Early Steps on Multiple Operations}

In [Fuh88] we have a first picture of a change operation that is not necessarily by a single input. It starts with contraction and, then, goes to revision.

\subsubsection{Multiple Contraction}

Fuhrmann claims that sometimes we need to withdraw more than one proposition of a belief set at the same time, proposing the name Multiple Contraction for this case.

Suppose that $K$ is a belief set and $A$ is a set of sentences to be retracted from $K$. Fuhrmann observes that the operation represented by $K-A$ brings two different interpretations. If an agent wants no sentence of $A$ to be implied by $K-A$, that is, $C n(K-A) \cap A=\varnothing$, then we have a (multiple) package contraction ${ }^{1}$ (denoted by ${ }_{-p}$ ). On the other hand, if an agent simply does not want $A$ to be a subset of the consequences of $K-A$, that is, $A \nsubseteq C n(K-A)$, then we have a (multiple) choice contraction (here denoted by $\left.-{ }_{c}\right)$. A contraction by a single sentence, thus, becomes a special case of multiple contraction (both choice and package).

\footnotetext{
${ }^{1}$ Originally, in [Fuh88], the package contraction was called meet contraction, receiving the name package only in [FH94].
} 


\subsubsection{Package Contraction}

Some properties of an operation of package contraction are described below:

$\left({ }_{p}\right.$-success): if $A \cap C n(\varnothing)=\varnothing$, then $A \cap C n\left(K{ }_{p} A\right)=\varnothing$

$\left(-{ }_{p}\right.$-inclusion): $K{ }_{-p} A \subseteq K$

(- $p$-uniformity): if for any sets $A$ and $B$ it holds that, $\forall K^{\prime} \subseteq K, A \cap C n\left(K^{\prime}\right) \neq \varnothing$ iff $B \cap C n\left(K^{\prime}\right) \neq \varnothing$, then $K{ }_{-p} A=K{ }_{p} B$

(- ${ }_{p}$-relevance): if $\alpha \in K \backslash\left(K{ }_{p} A\right)$, then $\exists K^{\prime}$ such that $K{ }_{p} A \subseteq K^{\prime} \subseteq K, K^{\prime} \cap C n(A)=\varnothing$ but $C n\left(K^{\prime} \cup\{\alpha\}\right) \cap A \neq \varnothing$.

Now, we are going to bring some tools for constructing a multiple package contraction. Generalizing the remainder set from Definition 1 to the package contraction case we have:

Definition 23. [Han89] $X \in K \perp_{P} A$ iff:

- $X \subseteq K$,

- $\operatorname{Cn}(X) \cap A=\varnothing$, and

- $\forall Y$ such that $X \subset Y \subseteq K$, it holds that $C n(Y) \cap A \neq \varnothing$.

As well as for the singleton case, we need a selection function, which will be a straightforward generalization of the one described in Definition 2:

Definition 24. [Han89] Let $K$ be a set of sentences. A selection function for $K$ is a function $\gamma$ such that for every set $A$ :

- if $K \perp_{P} A \neq \varnothing$, then $\gamma\left(K \perp_{P} A\right) \neq \varnothing$ and $\gamma\left(K \perp_{P} A\right) \subseteq K \perp_{P} A$

- if $K \perp_{P} A=\varnothing$, then $\gamma\left(K \perp_{P} A\right)=\{K\}$

Then, the operator can be constructed as follows:

Definition 25. [Han89] An operator $-_{p}$ is a partial meet package contraction iff for each theory $K$ there exists a selection function $\gamma$ such that:

$$
K-_{p} A=\bigcap \gamma\left(K \perp_{P} A\right)
$$

Theorem 11. [FH94] An operator - is a partial meet package contraction iff, for each theory $K$ and sets $A$ and $B$, it satisfies the following postulates: ${ }_{-}{ }_{p}$-success, ${ }_{-}{ }_{p}$-inclusion, ${ }^{-}{ }_{p}$-uniformity and ${ }_{p}$-relevance.

\subsubsection{Choice Contraction}

Some properties of an operation of package contraction are described below:

(- ${ }_{c}$-success): if $A \nsubseteq C n(\varnothing)$, then $A \nsubseteq C n\left(K-{ }_{c} A\right)$

$\left(-{ }_{c}\right.$-inclusion): $K-{ }_{c} A \subseteq K$

(- ${ }_{c}$-uniformity): if for any sets $A$ and $B$ it holds that, $\forall K^{\prime} \subseteq K, A \subseteq C n\left(K^{\prime}\right)$ iff $B \subseteq C n\left(K^{\prime}\right)$, then $K-_{p} A=K{ }_{p} B$ 
(- ${ }_{c}$-relevance): if $\alpha \in K \backslash\left(K-{ }_{c} A\right)$, then $\exists K^{\prime}$ such that $K{ }_{c} A \subseteq K^{\prime} \subseteq K, C n(A) \nsubseteq K^{\prime}=\varnothing$ but $\left(A \subseteq C n\left(K^{\prime} \cup\{\alpha\}\right)\right.$.

Now, we are going to bring some tools for constructing a multiple choice contraction. Generalizing the remainder set from Definition 1 to the choice contraction case we have:

Definition 26. [FH94] $X \in K \perp_{C} A$ iff:

- $X \subseteq K$,

- $A \nsubseteq C n(X)$, and

- $\forall Y$ such that $X \subset Y \subseteq K$, it holds that $A \subseteq C n(Y)$.

The selection function for this case works in the same way as for package (Definition 24). Then, the operator can be constructed as follows:

Definition 27. [FH94] An operator $-_{c}$ is a partial meet choice contraction iff for each theory $K$ there exists a selection function $\gamma$ such that:

$$
K-{ }_{c} A=\bigcap \gamma\left(K \perp_{C} A\right)
$$

Theorem 12. [FH94] An operator - is a partial meet choice contraction iff, for each theory $K$ and sets $A$ and $B$, it satisfies the following postulates: ${ }_{{ }_{c}}$-success, ${ }_{{ }_{c}}$-inclusion, ${ }_{-}{ }_{c}$-uniformity and $-{ }_{c}$-relevance.

For more properties, operators and relations about Multiple Contraction and the possibilities to reduce it or not to a single operation see [FH94, FSS03, Rei11].

\subsubsection{Multiple Revision}

Following [Fuh88], we also see the discussion of some properties of revision operations that receive as input more than one sentence simultaneously, named Multiple Revision. Analogously to Multiple Contraction, when the result of $K * A$ should imply everything in $A(A \subseteq C n(K * A))$ we have (multiple) package revision ${ }^{2}$ (denoted by ${ }_{p}$ ), while when the result of $K * A$ should contain some elements (but not necessarily all) of $A(C n(K *$ $A) \cap A \neq \varnothing)$, then we have (multiple) choice revision ${ }^{3}$ (here denoted by ${ }_{c}$ ).

In order to proceed with the generalization, we need the definition of set negation. Fuhrmann defined it this way: if $A$ is a set of formulas, $\neg A=\{\neg \alpha: \alpha \in A\}$.

Now, the package revision operation can be defined via a generalized version of the Levi Identity using package contraction:

$$
K{ }^{*} A=\left(K-{ }_{p} \neg A\right)+A
$$

By this approach, the properties of a package revision operation become dictated by the ones from package contraction operation.

\footnotetext{
${ }^{2}$ Again, in [Fuh88] this operation was denominated meet revision. The terminology "package revision" is used in [Han06, Spo10], based on the terminology from [FH94]. Another name found in the literature is bunch revision [Rot01].

${ }^{3}$ Hans Rott called this one pick revision [Rot01].
} 
Theorem 13. [Fuh88] If the operation of package (theory) revision is defined via Levi Identity from the operation of package contraction, then the following conditions hold for the package revision of any theory $K$ by any sets of sentences $A, A$ ':

1. (closure) $K *_{p} A$ is a theory;

2. (success) $A \subseteq K *_{p} A$;

3. (inclusion) $K *_{p} A \subseteq K+A$;

4. (vacuity) if $K \cap \neg A=\varnothing$ then $K+A \subseteq K{ }^{*} A$;

5. (extensionality) if $A$ and $A^{\prime}$ are pairwise equivalent ${ }^{4}$, then $K{ }^{*} p A=K{ }_{p} A^{\prime}$;

6. (conjunctive inclusion) $K{ }^{*} p\left(A \cup A^{\prime}\right) \subseteq\left(K{ }_{p} A\right)+A^{\prime}$;

7. (conjunctive vacuity) if $K \cap \neg A=\varnothing$, then $\left(K *_{p} A^{\prime}\right)+A \subseteq K *_{p}\left(A^{\prime} \cup A\right)$.

Still within the same thesis, the author claims that the operation of choice revision is less intuitive than package revision, making it difficult to find practical applications. If in a choice revision $K{ }^{*} c A$ an agent has to add at least one sentence from $A$, which ones should he choose? The principle of minimal change, already established for contractions, should prevail in this situation as well. But it can be the case that, whatever the sentences from $A$ that it decides to add to $K$, an incision on $K$ will be needed. So, according to the thesis, the agent should "retract only those sentences which are, by comparison, the least important (the most retractible) ones". Representing this idea using the Levi Identity, we have:

$$
K *_{c} A=\left(K-{ }_{c} \neg A\right)+A \cap\left\{\alpha: \neg \alpha \notin\left(K-{ }_{c} \neg A\right)\right\}
$$

where $\left[K-{ }_{c} \neg A\right]$ represents the choice contraction of $K$ by those elements of $\neg A$ that are "easiest to retract".

\subsubsection{Generalizing Grove's Result}

Sten Lindström introduces in [Lin91] a set of operations called infinitary belief revision. In his article, he explores nonmonotonic inference operations and, at the end, brings a connection between AGM revision and them. Nevertheless, in order to achieve total interdefinability between belief revision and nonmonotonic inference, it was necessary to support possibly infinite sets of propositions as input.

The axioms proposed are direct generalizations of the basic revision axioms presented in [Gär88], similarly to what was done in [Fuh88]. The main differences are that Lindström adds one for consistency and also joins the inclusion and vacuity postulates to form a new one called Expansion:

(consistency preservation) if $A$ is not inconsistent then $K * A$ is consistent.

(expansion) if $K \cup A$ is not inconsistent, then $K * A=K+A$.

\footnotetext{
${ }^{4}$ Two sets of sentences $A$ and $B$ are pairwise equivalent (modulo $\mathrm{Cn}$ ) just in case: $\forall \alpha \in A: \exists \beta \in B$ s.t. $C n(\alpha)=C n(\beta)$ and $\forall \beta \in B: \exists \alpha \in A$ s.t. $C n(\beta)=C n(\alpha)$.
} 
So, we have five basic axioms instead of six. He also gives four supplementary postulates:

(Chernoff) $K *_{p}\left(A \cup A^{\prime}\right) \subseteq\left(K *_{p} A\right)+A^{\prime}$;

(Arrow) if $\left(K *_{p} A\right)+A^{\prime}$ is consistent, then $\left(K *_{p} A\right)+A^{\prime}=K *_{p}\left(A \cup A^{\prime}\right)$.

(Aizerman) if $A \subseteq A^{\prime} \subseteq K{ }^{*} p$, then $K *_{p} A \subseteq K{ }^{*} A^{\prime}$.

(Gamma) $K *_{p}\left(\bigcap_{A \in F} C n(A)\right) \subseteq C n\left(\bigcup_{A \in F} K *_{p} A\right)$, where $F$ is a non-empty family of sets of sentences.

As can be seen, the first two ones are a generalization of Gärdenfors' supplementary postulates for singleton revision. Lindström states that Fuhrmann's postulates are equivalent to four of his basic postulates (i.e., excluding just consistency preservation) together with arrow. Actually, chernoff should also be part of this correspondence since it is equal to Fuhrmann's conjunctive inclusion.

In spite of the similarity between postulates, Lindström, in comparison with the AGM model, makes weaker assumptions about the underlying logic. He only supposes that it is a deductive logic (a finitary Tarski-style consequence relation), while the AGM framework, in addition, supposes supraclassicality and satisfaction of the deduction theorem ${ }^{5}$.

In order to complete his theory, he gives a theorem to show the definition of infinitary belief revision in terms of Grove's systems of spheres[Gro88]:

Theorem 14. ${ }^{6}$ [Lin91] Let * be a (multiple) belief revision operation. Then, * is a system of spheres-based revision iff it satisfies closure, success, extensionality, expansion, consistency preservation and arrow.

Then, the first one to state that Grove's model can be generalized to multiple revision was Lindström (although he did not prove it).

\subsubsection{Internal and External Revision for Belief Bases}

Hansson started to show the necessity and the rationality of multiple revision in [Han89, Han92]. When extending AGM revision for belief bases in [Han93], he generalized it to the multiple case at the same time. Considering the operation of revision obtained from the Levi Identity, in order to proceed with the generalization we need, for sets, an equivalent way of negating the input. However, unlike Fuhrmann that defined the negation of a set as the set formed by the negation of each sentence of the original set, Hansson took a different path.

In the Levi Identity, the original motivation to contract by $\neg \alpha$ was to extract a subset of the initial set that is consistent with $\alpha$. Let $X$ represent this subset. After this contraction by the negation of $\alpha$, we want $\neg \alpha \notin C n(X)$ to be true. Let us now apply this same interpretation for sets.

\footnotetext{
${ }^{5} \beta \in C n(A \cup\{\alpha\})$ iff $\alpha \rightarrow \beta \in C n(A)$.

${ }^{6}$ Originally, the theorem was shown split in two parts, one for each direction of the condition.
} 
Unlike single sentences, sets of sentences do not have negations. Suppose that we want to revise a belief base $B$ by $A$. So, we want to obtain for $A$ some set that behaves in relation to it in the same way as the negation of a sentence to that sentence itself. The idea to contract some sentences from $B$ (producing $X \subseteq B$ ) is to make it consistent with $A$, which means that $\perp \notin C n(X \cup A)$. By the compactness property of $C n$, this is equivalent to $\perp \notin C n\left(X \cup A^{\prime}\right)$ for every finite subset $A^{\prime}$ of $A$, which is also equivalent to $\perp \notin C n\left(X \cup \wedge A^{\prime}\right)$ for every finite subset $A^{\prime}$ of $A$ and, by the deduction property, equivalent to $\left(\bigwedge A^{\prime} \rightarrow \perp\right) \notin C n(X)$. Finally, we can derive that $\neg\left(\bigwedge A^{\prime}\right) \notin C n(X)$.

Therefore, in order to obtain $X$, we have to remove from $B$ the set that contains all sentences in the form $\neg\left(\bigwedge A^{\prime}\right)$ for some finite subset $A^{\prime}$ of $A$. So, considering our goal, this set can be considered as the negation of $A$ :

Definition 28. [Han91] For any set $A$ of sentences, $\neg A$ (the negation of $A$ ) is the set such that $\alpha \in \neg A$ iff $\alpha$ is either:

1. $\perp$;

2. a negation of some sentence in A;

3. a finite disjunction of sentences that are negations of elements of $A$.

So, $\neg A$ is the set formed by all the sentences that are either negations of elements of $A$ or (finite) disjunctions of such negations. If $A$ is finite, its negation can be simplified to a single sentence form:

Definition 29. [Han92] Let $A$ be a finite set of sentences. Then neg $(A)$ (the sentential negation of $A$ ) is defined as follows:

1. $\operatorname{neg}(\varnothing)=\perp$;

2. if $A$ is a singleton, $A=\{\alpha\}$, then $n \operatorname{eg}(A)=\neg \alpha$;

3. if $A=\left\{\alpha_{1}, \ldots, \alpha_{n}\right\}$ for some $n>1$, then $\operatorname{neg}(A)=\neg \alpha_{1} \vee \neg \alpha_{2} \vee \ldots \vee \neg \alpha_{n}$.

As defined above, the sentential negation of a finite non-empty set $A$ is the disjunction of the negation of the sentences from $A$.

Before defining the operation itself, Hansson also brings some analysis on selection functions. A function $\gamma$ proposed as in Definition 24 is referred to as a one-place selection function, which is specific for a given belief base $B$ and that, for each set $A$, selects a non-empty subset of $B \perp A$ (unless $B \perp A$ is empty). If $B \neq B^{\prime}$, then $\gamma$ is not a one-place selection function for $B^{\prime}$. Thus, after a contraction/revision process we have a new belief base but not a new selection function that would allow us to proceed with further belief changes. For this purpose, a more general function was defined:

Definition 30. [Han93] $A$ two-place selection function is a function $\gamma$ such that, for each subset $B$ of $\mathcal{L}, \gamma(B)=,\gamma_{B}()$ is a one-place selection function for $B$. A two-place selection function $\gamma$ is unified iff, for all subsets $B_{1}$ and $B_{2}$ of $\mathcal{L}$, if $B_{1} \perp A_{1}=B_{2} \perp A_{2} \neq \varnothing$, then $\bigcap \gamma_{B_{1}}\left(B_{1} \perp A_{1}\right)=\bigcap \gamma_{B_{2}}\left(B_{2} \perp A_{2}\right)$.

As observed by the author, the one-place selection functions produced by a unified twoplace selection function coincide for all arguments. For convenience, the first argument 
of a unified two-place selection function can be omitted (such that $\bigcap \gamma(B \perp A)$ is a shorter form of $\left.\bigcap \gamma_{B}(B \perp A)\right)$.

Taking the definitions of remainder set, set negation and selection functions, Hansson defines multiple internal revision:

Definition 31. [Han93] Let $\gamma$ be a one-place selection function for a subset $B$ of $\mathcal{L}$. Then the operator ${ }^{*}{ }_{\gamma}$ is the operator of multiple internal package partial meet base revision for $B$ generated by $\gamma$ iff for all finite subsets $A$ of $\mathcal{L}$ :

$$
B{ }_{\gamma} A=\bigcap \gamma(B \perp\{n e g(A)\}) \cup A
$$

We can generalize this construction by applying a two-place selection function instead of a one-place kind. Then, for all subsets $B$ of $\mathcal{L}$ and all finite subsets $A$ of $\mathcal{L}$, the same identity holds (with due exchange).

Then, it is possible to characterize it axiomatically:

Theorem 15. [Han93] The operator ${ }^{*}$ is an operator of multiple internal package partial meet base revision for a belief base $B$ iff it satisfies:

(success) $A \subseteq B * A$

(inclusion) $B * A \subseteq B \cup A$

(weak consistency) $B * A$ is consistent if $A$ is consistent

(uniformity) If for all $B^{\prime} \subseteq B, B^{\prime} \cup A$ is inconsistent iff $B^{\prime} \cup C$ is inconsistent, then $B \cap(B * A)=B \cap(B * C)$.

(relevance) If $\alpha \in B$ and $\alpha \notin B * A$, then there is some $B^{\prime}$ such that $B * A \subseteq B^{\prime} \subseteq B \cup\{A\}$, $B^{\prime}$ is consistent but $B^{\prime} \cup\{\alpha\}$ is inconsistent.

In comparison with Gärdenfors' postulates for singleton revision, weak consistency, inclusion and success are direct generalizations (except for the logical closure for inclusion). Uniformity substitutes extensionality since base revision considers syntax and not only semantic contents. Relevance is required to ensure minimality, i.e., if a sentence was removed from the original base then it had a participation in the inconsistency problem between the base and the new beliefs. Also, these postulates are straightforward generalizations of the postulates for singleton base revision presented in Section 2.6.2.1.

Observation 4. If an operator * for a belief base B satisfies success, inclusion and relevance, then it satisfies:

(vacuity) If $B \cup A \forall \perp$ then $B * A=B \cup A$

Proof. This proof can be straightforwardly adapted from the version for singleton revision in [Han99b].

While for internal revision we first have a contraction and then the expansion, for external revision we take the inverse way: we first get the union of the base and the input and then perform a contraction by the sentential negation of the input: 
Definition 32. [Han93] Let $\gamma$ be a two-place selection function. The operator of multiple external package partial meet base revision generated by $\gamma$ is the operator $\pm_{\gamma}$ such that for all subsets $B$ of $\mathcal{L}$ and all finite subsets $A$ of $\mathcal{L}$ :

$$
B \pm_{\gamma} A=\bigcap \gamma_{B \cup A}((B \cup A) \perp\{n e g(A)\})
$$

In this case, we need a selection function of the two-place kind because if we had a one-place selection function for $B$ it would not work for the contraction of $B \cup A$ by $n e g(A)$. That is why we need a more general one.

The axiomatic characterization follows below:

Theorem 16. [Han93] The operator ${ }^{*}$ is an operator of multiple external package partial meet base revision iff it satisfies weak consistency, inclusion, relevance, success and, in addition:

(weak uniformity) If $A$ and $C$ are subsets of $B$ and it holds that for all $B^{\prime} \subseteq B$ that $B^{\prime} \cup A$ is inconsistent iff $B^{\prime} \cup C$ is inconsistent, then $B * A=B * C$.

(pre-expansion) $(B \cup A) * A=B * A$

As can be seen, two of the postulates are different from those of the previous theorem. Weak uniformity is a constraint on uniformity to the particular case of revision by a subset of the belief base, while Pre-expansion requires that a revision by a set should return the same outcome as the sequence of first expanding and then revising by the same set. To see why uniformity does not hold in general for external revision or why internal and external revision are really different operations, see [Han93].

\subsubsection{Multiple Package Partial Meet Revision}

As shown in the second condition of Theorem 13, the generalization of the revision operation inherits an important characteristic already present in the original definition: the input set has priority over the sentences to be revised. Following this approach, we have in [Fuh97] a further exploration of the topic, bringing us a construction. Fuhrmann addresses the issue focusing on the package variety and shows how the operations of revision and contraction can be inter-definable.

\subsubsection{Opening the Set}

Following the partial meet approach, when we have arbitrary sets of sentences $K$ and $A$ and need to revise $K$ in order to consistently incorporate $A$ completely, we can first find all the subsets of $K$ that are maximally compatible with $A$ (denoted by $K \downarrow_{p} A$ ), which form a generalized version of the remainder set for multiple operations in revision ${ }^{7}$ (following the package approach):

Definition 33. [Fuh97] $X \in K \downarrow_{p}$ A iff:

- $X \subseteq K$;

- $X \cup A$ is consistent;

\footnotetext{
${ }^{7}$ In [Fuh97], this generalized remainder set is called $K$ open for $A(K$ op $A)$.
} 
- $\forall X^{\prime}$ such that $X \subset X^{\prime} \subseteq K$ then $X^{\prime} \cup A$ is inconsistent.

So, if you have a consistent set $A, K \downarrow_{p} A$ is the set of the maximal $K$-subsets consistent with $A$.

It is interesting to note that, although the AGM framework was initially defined to work with singleton inputs, the original definition of remainder set, given in [AM81] for contraction, considered the input to be a set of propositions. The difference between Fuhrmann's definition given here and the one from Alchourrón and Makinson is that, while the former is concerned about consistency, the latter cares about implication, but both of them in a maximal way. Also, the generalized version does not depend on negation. At the same time, it is possible to establish the following relation:

Observation 5. [Fuh97] Given a belief set $K$ and a finite set of sentences $A$, it holds that $K \downarrow_{p} A=K \perp n e g(A)$.

Proof: First, let us show that $K \perp n e g(A) \subseteq K \downarrow_{p} A$. Let $X \in K \perp n e g(A)$. Then $X \subseteq K$ and, given that $A=\left\{\alpha_{1}, \ldots, \alpha_{n}\right\}$ for some $n>=1, X \forall \neg \alpha_{1} \vee \ldots \vee \neg \alpha_{n}$. Thus, none of the negated sentences from $A$ are implied by $X$. Therefore, $X \cup A$ is consistent. Given the maximality of $X$, we conclude that $X \in K \downarrow_{p} A$. The converse property is analogous. Therefore, $K \downarrow_{p} A=K \perp n e g(A)$.

\subsubsection{Completing the Construction}

After defining the generalized remainder set, Fuhrmann assumed that he had some ways to get from $K \downarrow_{p} A$ some most preferred elements, representing this by a selection function ${ }^{8} \gamma$ in the same way as in Definition 24. Considering $\bigcap \gamma\left(K \downarrow_{p} A\right)$ as the intersection of the most preferred elements, we can find the revised $K$ apt to consistently incorporate $A$, which gives us the way to represent the multiple package partial meet revision:

$$
K *_{p} A=\bigcap \gamma\left(K \downarrow_{p} A\right) \cup A
$$

\subsubsection{Properties}

The following result was obtained for this package revision:

Theorem 17. [Fuh97] Let ${ }_{p}$ be a partial meet package contraction operation (Definition 12) and let * be the operation generated from ${ }_{p}$ by the Levi Identity $\left(K *_{p} A\right)$. Then * satisfies the following conditions:

1. (inclusion) $K * A \subseteq K \cup A$

2. (success) $A \subseteq K * A$

3. (weak consistency) If $A \forall \perp$, then $K * A \forall \perp$

4. (congruence) If $\forall K^{\prime} \subseteq K$ it holds that $\neg A \cap C n\left(K^{\prime}\right) \neq \varnothing$ iff $\neg C \cap C n\left(K^{\prime}\right) \neq \varnothing$ then $K * A=K * C$

\footnotetext{
${ }^{8}$ In [Fuh97], this selection function is called choice function, given that it chooses some elements from the remainder set.
} 
5. (relevance) If $\alpha \in K \backslash K * A$ then there exists $X$ such that $(K * A) \cap K \subseteq X \subseteq K, X \cup A$ is consistent but $X \cup A \cup\{\alpha\}$ is inconsistent.

In comparison to Theorem 15 from Hansson, they are equivalent since the postulate of congruence is equivalent to uniformity. On the other hand, in comparison to Theorem 13 from Fuhrmann, this last one maintains the success and inclusion postulates (although removing the closure from inclusion), substitutes extensionality for congruence, adds consistency and relevance and excludes vacuity and closure. This time, Fuhrmann presented the vacuity postulate just as an observation, derived from success, inclusion and relevance. Closure was applied only when dealing with belief sets (as will be seen in Section 3.1.5.5). This set of postulates is smaller than Fuhrmann's original one because it is sufficient to characterize the given construction:

Theorem 18. [Fuh97] If an operator * satisfies the five postulates from Theorem 17, then there exists a selection function $\gamma$ for $K$ such that $K * A=\bigcap \gamma\left(K \downarrow_{p} A\right) \cup A$.

\subsubsection{Connecting with Contraction}

The author also explored how the opening operation can be defined from the package remainder operation for contraction, bringing a relation between their properties. We are going to show in more details how to achieve the definition of the package revision operation shown in Section 3.1.2.

In order to start, Fuhrmann follows Hansson's suggestion to define set-negation, where the negation of $A$ would be the negated conjunctions of all finite nonempty subsets of A:

$$
\operatorname{neg}(A)=\left\{\neg \bigwedge A^{\prime}: A^{\prime} \subseteq_{f} A\right\}^{9}
$$

The only difference between Definition 28 and this one is that the former specifies the case for empty sets.

Applying this to Definitions 23 and 33, we can have an equivalence:

$$
K * A=\bigcap \gamma\left(K \downarrow_{p} A\right) \cup K=\bigcap \gamma(K \perp \neg A) \cup A
$$

From the definition of package contraction using remainder sets, we have:

$$
K-\neg A=\bigcap \gamma(K \perp \neg A)
$$

Therefrom, these last two equations lead to a generalisation of the Levi identity:

$$
K * A=(K-\neg A) \cup A
$$

So, the properties for package contraction can be rendered to properties for package revision.

\subsubsection{Matching Revision}

The revision operation defined so far should retain the properties of openness and closedness of belief sets, i.e., it should obey the principle of categorial matching defined in

${ }^{9} A^{\prime} \subseteq_{f} A$ iff $A^{\prime}$ is a nonempty and finite subset of $A$. 


\section{[GR95]:}

"The representation of a belief state after a belief change has taken place should be of the same format as the representation of the belief state before the change."

From this principle, theories map onto theories and belief bases map onto belief bases. Therefore, the operation should be general enough so that it is not characterized only for belief sets or belief bases. In the way the operation was defined so far,it is not true that in general a possible closedness is preserved. Therefore, Fuhrmann called the operation prerevision and established an operation of matching revision, a revision that is in accordance with the principle of categorial matching:

$$
K \star A= \begin{cases}C n(K * A) & \text { if } K=C n(K) \\ K * A & \text { otherwise }\end{cases}
$$

The observation that follows indicates that the operation $\star$ is in practice a simple adaptation of pre-revision.

Observation 6. [Fuh97] If * satisfies the conditions 1-5 from Theorem 17, then $\star$ satisfies conditions 2-5 and in addition:

6. (inclusion) $K \star A \subseteq C n(K \cup A)$ and $K \star A \subseteq K \cup A$ if $K \neq C n(K)$

7. (closure) If $K=C n(K)$ then $K \star A=C n(K \star A)$

Observation 7. [Fuh97] If $\star$ satisfies the conditions 2-7 from Observation 6, then there exists a revision operator * satisfying conditions $1-5$ such that

$$
K \star A= \begin{cases}C n(K * A) & \text { if } K=C n(K) \\ K * A & \text { otherwise }\end{cases}
$$

\subsubsection{Reduction}

In multiple operations, one of the questions that emerge is whether it is possible to reduce them to operations by singletons. Based on the search of definitions for set negation (as shown in Section 3.1.4), Hansson obtained the result below:

Observation 8. [Han91] Let $-_{p}$ be an operation of multiple partial meet contraction. It then holds for all sets $K$ and all finite sets $A$ that $K-_{p} \neg A=K-_{p} n e g(A)$.

For package revision, Fuhrmann formalized the following reduction result:

Lemma 1. [Fuh97] For every belief set $K$ and finite set $A, K * A=K * \bigwedge A$

It means that, in the finite case, it is possible to reduce a multiple revision to a revision by a singleton (the conjunction of all the elements of the input). Indeed, this property is confirmed by other works available in the literature. Hans Rott, for example, claims that "accepting all sentences of a finite set simultaneously essentially means accepting their conjunction”[Rot01]. 
However, regarding belief bases, there is a logical drawback for this reduction. For comparison purposes, suppose that, instead of revising by a set $\{\alpha, \beta\}$ (a collection of items of information), an agent decides to revise by the conjunction $\alpha \wedge \beta$ (a single item of information). As observed in [DJ12], although the two options have the same logical content (since they imply precisely the same formulas), revising by $\{\alpha, \beta\}$ should result in a belief state such that, if there is no known link between $\alpha$ and $\beta$, then if $\beta$ were afterwards found out as not true, then $\alpha$ should still be considered as true. That is why, the authors say, revision by a conjunction and revision by the set of conjuncts should be managed separately.

In addition, since $n e g(A)$ is defined as the disjunction of the negation of the sentences from $A$, for a finite set $A$, this reduction property works only for languages that allow logic conjunction/disjuction and negation of sentences. It is not the case for many Description Logics and for Horn Logic, for example, which limits the application of the property.

\subsection{Infinitary Belief Change}

Zhang [Zha96] observed that, although multiple revision was not an unprecedented topic, there was still a need for a general framework towards belief revision by sets of sentences, especially infinite sets, a topic that was retaken in [ZCZC97, ZF01]. One of the reasons for this claim is that the relations between contraction and revision (through Levi and Harper identities) are not valid anymore when we deal with infinite inputs, which can happen, for example, when the underlying language is expanded to first-order logic. So the purpose of the articles was to extend the AGM framework (its axiomatization and modeling) to a more general one in order to include revision by any set of sentences.

\subsubsection{General Contraction}

In the traditional idea of contraction given by the AGM theory, contracting $K$ by $A$ means removing $A$ and some other beliefs from $K$ so that $A$ is not implied anymore. However, Dongmo Zhang proposes a new operator called general contraction (denoted by $\ominus$ and also called set contraction in [ZF01]) whose purpose is to delete some sentences from $K$ so that the remaining subset is consistent with $A$ and logically closed. Zhang and Foo [ZF01] observe that, even though it is different from the initial purpose of contraction, this new operator elucidates a significant intuition about contraction: we give up our beliefs when they conflict with some new information (point discussed in Section 2.4).

Eight postulates were proposed for this kind of contraction:

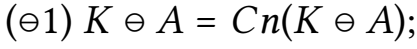

$(\ominus 2) K \ominus A \subseteq K$;

( $\ominus 3)$ if $K \cup A$ is consistent, then $K \ominus A=K$;

( $\ominus 4)$ if $A$ is consistent, then $A \cup(K \ominus A)$ is consistent.

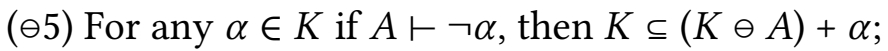

(ө6) if $C n\left(A_{1}\right)=C n\left(A_{2}\right)$, then $K \ominus A_{1}=K \ominus A_{2}$; 
(ө7) $K \ominus A \subseteq K \ominus(A \vee B)+A^{10}$

( $\ominus 8)$ If $A \cup(K \ominus(A \vee B))$ is consistent, then $K \ominus(A \vee B) \subseteq K \ominus A$.

According to [Zha96], postulates $(\ominus 1)-(\ominus 4)$ and $(\ominus 6)$ are obvious (given that they are direct generalizations of AGM postulates). ( $\ominus 5)$ is a generalization of Gärdenfors' postulate of recovery [Gär88] (although a bit stronger) and the last two are supplementary postulates.

General contraction points to the first step of internal revision, when a contraction is performed in order to make the original set consistent with the input. Following this vision of contraction, its use comes down to an auxiliary tool to build revision. Zhang and Foo showed that the relations between sentence revision and AGM contraction (Levi and Harper identities) are, with suitable adaptations, also valid between multiple revision and general contraction. According to the authors, that is why they focused on set contraction in their article, i.e., multiple revision can be derived through the identity.

\subsubsection{Nice-Ordering Partition and Nice-Well-Ordering Partition}

In [Zha96], Zhang developed a modeling for multiple revision based on epistemicentrenchment ${ }^{11}$. He argues that there is a more basic concept than epistemic entrenchment: degree of belief. The reason for that is because it is not always clear why or when a belief has a higher degree of epistemic entrenchment than another, that is, it is unrealistic to demand from an agent an arrangement of all its knowledge into a total ordering.An agent that believes some sentences accepts them with different degrees of reliability. Then, it is possible to distribute our beliefs into finite or infinitely many groups depending on the degrees in which we believe them, being possible to organize these groups into a total-ordering. That is why he proposes a structure called total-ordering partition (TOP), whose arrangement depends only on our instinctive sense. If the partition is rearranged in order to satisfy some logical constraints we have a nice-ordering partition (NOP). The relation between NOP and epistemic entrenchment was studied and, in brief, it is possible to say that the latter can be seen as particular case of the former in some sense.

Definition 34. [Zha96] For any belief set $K$, let $\mathcal{P}$ be a partition ${ }^{12}$ of $K$ and $<$ a total-ordering relation on $\mathcal{P}$. The triple $\Sigma=(K, \mathcal{P},<)$ is called a TOP of $K$.

For any $P \in \mathcal{P}$, if $\alpha \in P, P$ is called the rank of $\alpha$, denoted by $b(\alpha)$.

The ordering of the rank is not a direct relation with the degree of belief. Actually, the converse ordering of the belief degree is the ordering of rank, which means that the higher the belief degree of a sentence is, the less rank of the sentence will be.

Definition 35. [Zha96] A TOP $\Sigma=(K, \mathcal{P},<)$ is a NOP if it satisfies the following logical constraint: if $\alpha_{1}, \ldots, \alpha_{n} \vdash \beta$, then $\sup \left\{b\left(\alpha_{1}\right), \ldots, b\left(\alpha_{n}\right)\right\} \geq b(\beta)$.

\footnotetext{
${ }^{10} A \vee B=\{\alpha \vee \beta: \alpha \in A \wedge \beta \in B\}$

${ }^{11}$ In a general way, epistemic entrenchment is a special kind of total preorder on sentences.

${ }^{12}$ A partition of a set $K$ is a disjoint family $\mathcal{P}$ of subsets of $K$ such that $K=\bigcup\{P: P \in \mathcal{P}\}$.
} 
In the above definition, if the premise of the logical constraint holds it means that the degree of belief of $\beta$ is not less than the minimum degree of belief of $\alpha_{1}, \ldots, \alpha_{n}$.

Using these new concepts, we are given an explicit construction for multiple contraction functions:

Definition 36. [Zha96] Let $\Sigma=(K, \mathcal{P},<)$ be a NOP of a belief set $K$. Define a function $\ominus$ called NOP contraction over $K$ as follows: for any $A$ :

- if $A \cup K$ is consistent, then $K \ominus A=K$;

- otherwise, $B \in K \ominus A$ iff $B \in K$ and there exists $C \in K$ such that $A \vdash \neg C$ and: $\forall D \in K(C \vdash D \wedge A \vdash \neg D \rightarrow(b(C) \vee B<b(D) \vee \vdash C \vee B))$

Theorem 19. [Zha96] If $\ominus$ is a NOP contraction over $K$, then it satisfies $(K \ominus 1)-(K \ominus 8)$.

Then, in [Zha96] we have a theoretical constructive approach based on a well-ordering partition:

Definition 37. [Zha96] A NOP $\Sigma=(K, \mathcal{P},<)$ of $K$ is called a nice-well-ordering partition (NWOP) of $K$ if $<$ is a well-ordering relation ${ }^{13}$ on $\mathcal{P}$.

A contraction generated by NWOP is given and used to establish a method to deal with general belief revision. Before defining it, we need an additional definition. When $\Delta \subseteq K$, we have $\Delta_{P}=\Delta \cap P$ and $\Delta_{\leq P}=\bigcup_{Q \leq P} \Delta_{Q}$.

Definition 38. [Zha96] Let $\Sigma=(K, \mathcal{P},<)$ be a total ordering partition of $K$ and $F$ be an arbitrary set of sentences. $K \downarrow F$ is defined as the family of all subsets $\Delta=\bigcup_{P \in \mathcal{P}} \Delta_{P}$, of $K$, where for any $P \in \mathcal{P}, \Delta_{P}$ is a maximal subset of $P$ such that $\Delta_{\leq} \cup F$ is consistent.

A constructive approach to contraction generated by NWOP was, then, proposed:

Definition 39. [Zha96] Let $\Sigma=(K, \mathcal{P},<)$ be an NWOP of a belief set $K$ and $A$ an arbitrary set of sentences. We define $\theta^{<}$, called NWOP contraction, as follows. If $A$ is inconsistent, then $K \theta^{<} A=K$. If $A$ is consistent, then $K \theta^{<} A=\bigcap K \downarrow A$.

Theorem 20. [Zha96] Let $\Sigma=(K, \mathcal{P},<)$ be an NWOP of $K$ and $A$ be an arbitrary set of sentences. Then $K \ominus A=K \ominus^{<} A$.

In [ZCZC97] the authors claim that in [Zha96] we have a complete extension of AGM's postulates for belief changes but without a representation theorem to the framework proposed. So, in their paper they provide two representation theorems for general contraction and, in addition, a new property called Limit Postulate in order to specify properties of infinite belief changes.

\subsubsection{New Properties}

In [ZCZC97] the authors revisit the postulates for general contraction defined in [Zha96] and change some points.

\footnotetext{
${ }^{13}$ An ordering $\leq$ is a well-ordering relation on $X$ iff each non-empty subset $X^{\prime} \subseteq X$ has a $\leq$-least element, i.e., there exists $\alpha \in X^{\prime}$ such that $\alpha \leq \beta$ for all $\beta \in X^{\prime}$.
} 
As cited above, they state that $(\ominus 5)$ appears to be stronger than the original recovery postulate. They provide, then, an equivalent property:

Lemma 2. [ZCZC97] If $\ominus$ satisfies $(\ominus 1)-(\ominus 4)$ then $(\ominus 5)$ is equivalent to the Saturation property:

$(\ominus S a t) \quad(K \ominus A+A) \cap K \subseteq K \ominus A$

Saturation establishes the reducibility between contraction and revision (see Theorem 25 below), being an implicit condition for set revision.

Regarding $(\ominus 6)$, although it is similar to AGM extensionality postulate, it was considered too weak in relation to what is expected. Then, a stronger version was suggested:

$\left(\ominus 6_{s}\right)$ If $\forall \alpha \in K$ we have that $A \cup\{\alpha\}$ is inconsistent iff $B \cup\{\alpha\}$ is inconsistent, then $K \ominus A=K \ominus B$.

$\left(\ominus 6_{s}\right)$ implies ( $\left.\ominus 6\right)$ but for the converse we need $(\ominus 7)$ and $(\ominus 8)$.

The authors in [ZCZC97] also state that postulates $(\ominus 7)$ and $(\ominus 8)$ are non-intuitive and suggest the adoption of two equivalent ones by doing a small improvement:

$$
\begin{aligned}
& \left(\ominus 7^{\prime}\right) \text { if } A_{1} \subseteq A_{2} \text {, then } K \ominus A_{2} \subseteq\left(K \ominus A_{1}\right)+A_{2} \\
& \left(\ominus 8^{\prime}\right) \text { if } A_{1} \subseteq A_{2} \text { and } A_{2} \cup\left(K \ominus A_{1}\right) \text { is consistent, then } K \ominus A_{1} \subseteq K \ominus A_{2} .
\end{aligned}
$$

For the rest of this section 3.2, postulates $(\ominus 1)-(\ominus 5)$ and $\left(\ominus 6_{s}\right)$ will be called the basic postulates for general contraction, while ( $\left.\ominus 7^{\prime}\right)$ and $\left(\ominus 8^{\prime}\right)$ will be considered the supplementary postulates.

\subsubsection{Partial Meet Model}

Zhang et. al. [ZCZC97] developed a Partial Meet model for the general contraction. For any two sets $K$ and $A$, they defined the remainder set $K \downarrow_{p} A$ in the same way as the remainder set for multiple revision defined by Fuhrmann in [Fuh97] (Definition 33). The respective selection function works in the same traditional way (Definition 24). So, it is possible to define the operation:

Definition 40. [ZCZC97] An operation $K \ominus$ is a partial meet contraction over $K$ iff there exists a selection function $\gamma$ such that for any set $A$ :

$$
K \ominus A= \begin{cases}K & \text { if } A \text { is inconsistent } \\ \bigcap \gamma\left(K \downarrow_{p} A\right) & \text { otherwise }\end{cases}
$$

Theorem 21. [ZCZC97] For any belief set $K, \ominus$ satisfies all the basic postulates for general contractions iff it is a partial meet contraction.

\subsubsection{The Limit Postulate}

When the input is not finite, the postulates for general contractions are not enough to uniquely establish a NOP contraction as a general contraction function, which demands 
an additional property to characterize the relation between a belief set contracted by an infinite set of sentences and by its finite subsets.

A plausible idea is to suppose that the contraction by an infinite set is a limiting case of the ones by its finite subsets. Let $\bar{A}$ be a finite subset of an infinite set $A$. So the Limit Postulate $(K \ominus L P)$ for general contraction can be defined as follows:

$$
K \ominus A=\bigcup_{\bar{A} \subseteq f} \bigcap_{\substack{\bar{A}^{\prime} \subseteq f \\ \bar{A} \subset \bar{A}^{\prime}}} K \ominus \bar{A}^{\prime}
$$

In words, $\beta \in K \ominus A$ iff there exists a finite subset $\bar{A}$ of $A$ such that for each finite subset $\bar{A}^{\prime}$ of $C n(A)$, if $\bar{A} \subseteq \bar{A}^{\prime}$, then $\beta \in K \ominus \bar{A}^{\prime}$.

Theorem 22. [ZCZC97] If $\ominus$ is a NOP contraction function, then $\ominus$ satisfies ( $K \ominus L P)$.

Theorem 23. [ZCZC97] Let $\ominus$ be a general contraction function over K. If $\ominus$ satisfies $(K \ominus L P)$, then there exists a NOP $\Sigma=(K, \mathcal{P},<)$ of $K$ such that $\ominus$ is exactly the NOP contraction generated by $\Sigma$.

Theorem 24. [ZCZC97] For any belief set $K$, $\ominus$ satisfies $(K \ominus 1)-(K \ominus 8)$ and $(K \ominus L P)$ iff $\ominus$ is a NOP contraction over $K$.

The proposition below demonstrates that the Limit Postulate implies a result given by Peppas [Pep96] (see Theorem 27) on the reduction from multiple revision to sentence revision when the belief set can be modeled by a finite sphere model:

Proposition 1. [ZF01] If $\ominus$ satisfies the postulates $(\ominus 1)-(\ominus 6)$ and $\left(\ominus 7^{\prime}\right)-\left(\ominus 8^{\prime}\right)$, then $(\ominus L P)$ is equivalent to the following condition:

$$
K \ominus A=\left(\bigcap_{\bar{A} \subseteq_{f} C n(A)} K \ominus \bar{A}+A\right) \cap K
$$

\subsubsection{Constructing Revision}

General revision (or set revision), denoted by $\otimes$, can be defined in terms of general contraction analogously to the Levi Identity (and vice-versa via the Harper Identity):

$($ Def $\otimes) K \otimes A=(K \ominus A)+A$

$($ Def $\ominus) K \ominus A=C n((K \otimes A) \cap A)$

The set of postulates usually used to characterize general/multiple revision is an adaptation between the ones defined by Fuhrmann [Fuh88] and the ones given by Lindström [Lin91]:

$(K \otimes 1) K \otimes A=C n(K \otimes A) ;$

$(K \otimes 2) A \subseteq K \otimes A ;$

$(K \otimes 3) K \otimes A \subseteq K+A$

$(K \otimes 4)$ if $K \cup A$ is consistent, then $K+A \subseteq K \otimes A$;

$(K \otimes 5) K \otimes A$ is inconsistent iff $A$ is inconsistent; 
$(K \otimes 6)$ if $C n\left(A_{1}\right)=C n\left(A_{2}\right)$, then $K \otimes A_{1}=K \otimes A_{2}$;

$(K \otimes 7) K \otimes\left(A_{1} \cup A_{2}\right) \subseteq\left(K \otimes A_{1}\right)+A_{2} ;$

$(K \otimes 8)$ If $A_{2} \cup\left(K \otimes A_{1}\right)$ is consistent, then $\left(K \otimes A_{1}\right)+A_{2} \subseteq K \otimes\left(A_{1} \cup A_{2}\right)$.

Theorem 25. [Zha96] If the general contraction (revision) function $\ominus(\otimes)$ satisfies (K $\ominus 1)$ $(K \ominus 8)((K \otimes 1)-(K \otimes 8))$, then the general revision (contraction) function $\otimes(\ominus)$ obtained from $D e f \otimes(D e f \ominus)$ satisfies $(K \otimes 1)-(K \otimes 8)((K \ominus 1)-(K \ominus 8))$.

Considering infinite inputs, according to [ZCZC97] a corresponding assumption for general revision $(K \otimes L P)$ is possible to be obtained in terms of $(\operatorname{Def} \otimes)$ :

$$
K \otimes A=\bigcup_{\bar{A} \subseteq_{f} A} \bigcap_{\substack{\bar{A}^{\prime} \subseteq_{f} C(A) \\ \bar{A} \subset \bar{A}^{\prime}}} K \otimes \bar{A}^{\prime}
$$

In [ZF01] it is proven that the Limit Postulate is enough to complete the fully characterization of general belief change operations. The corresponding version of Proposition 1 for set revision is as follows:

Proposition 2. [ZF01] If $\otimes$ satisfies the postulates $(K \otimes 1)-(K \otimes 8)$, then $(K \otimes L P)$ is equivalent to the following condition:

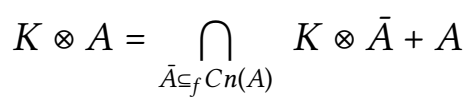

The results presented in [ZCZC97] both for general contraction and revision gives a groundwork for exploring the link between non-monotonic reasoning and multiple belief revision, a topic investigated in [ZCZL97], where the authors proposed a rational nonmonotonic system and provided two representation theorems relating Multiple Revision and their system. So, it is a generalization of the previous work of Gärdenfors, Makinson and Rott [MG91, GR95] on the relationship between belief revision and non-monotonic inference operations.

According to [ZF01], the Limit Postulate for revision, due to its equivalence to a property of non-monotonic reasoning called Finite Supracompactness [ZCZL97], is called the compactness of belief revision.

\subsubsection{Connection with MaxNon's}

It is possible to establish a relation between the general contraction operation and Maximal Non-Implying Subsets as defined by Matos et. al.:

Definition 41. [MGSW19] Let $K$ be a belief set, $\alpha$ a sentence and $\Phi$ a set of static sentences (i.e., which should be preserved in any operation). The set of maximal $\alpha$-non-implying subsets of $K$ with respect to $\Phi$, denoted by $\operatorname{MaxNon}(K, \alpha, \Phi)$, is such that $X \in \operatorname{MaxNon}(K, \alpha, \Phi)$ iff $X \subseteq K, \alpha \notin C n(\Phi \cup X)$, and there is no $Y$ such that $X \subset Y \subseteq K$ and $\alpha \notin C n(\Phi \cup Y)$.

By the above definition, the candidate elements to represent $K \ominus A$ can be obtained from $\operatorname{MaxNon}(K,\{\perp\}, A)$. 


\subsection{More on Systems of Spheres}

After the initial work developed by Lindström [Lin91], Peppas, Koutras and Williams explored more topics of the problem. Peppas studied some smoothness conditions on systems of spheres and their connection with multiple revision, giving also a constructive model for multiple revision based on systems of spheres along with a representation result. Together with Koutras and Williams, he continued the issues addressed in [ZF01] analyzing some problems about the limit postulate and additional constraints. Meng and Li [ML14, ML15] explored some open problems left by Peppas, using for that a topological approach.

\subsubsection{The Limit Assumption}

A specific smoothness condition satisfied by a total preorder on possible worlds (essentially, condition S4) is called the limit assumption, a name given by Lewis [Lew73]. Its central role is to ensure that for every consistent sentence $\varphi$ there is always a most-plausible $\varphi$-world.

The smoothness conditions considered by Peppas is his articles are actually variants of the limit assumption.

\subsubsection{Well Behaved Functions}

Peppas started his work by analyzing the aspects of well orderedness on systems of spheres:

Definition 42. [Pep96, Pep04] A system of spheres $\mathrm{S}$ is well ordered with respect to set inclusion iff it satisfies:

$(S W)$ Every nonempty subset of S has a smallest element with respect to $\subseteq$.

(SW) is stronger than (S4). With this definition, it is possible to define a class of revision functions:

Definition 43. [Pep96, Pep04] A revision function * is well behaved iff it can be constructed by well ordered systems of spheres.

In addition, a new strengthening of condition (S4) and a comparison result with (SW) are given:

$(S V)$ For every nonempty set $\mathcal{A}$ of consistent complete theories of $\mathcal{L}$, there exists a smallest sphere in $\mathrm{S}$ intersecting $\mathcal{A}$.

Lemma 3. [Pep96, Pep04] Let $\mathrm{S}$ be a system of spheres. $\mathrm{S}$ satisfies (SW) iff it satisfies (SV).

The extension of the construction based on systems of spheres to multiple revision can be defined as follows. For a consistent set of sentences $A, C_{\mathrm{S}}(A)$ is defined as the smallest 
sphere in $\mathrm{S}$ intersecting $[A]$, and $f_{\mathrm{S}}(A)$ is defined as the set $f_{\mathrm{S}}(A)=[A] \cap C_{\mathrm{S}}(A)$. Then:

Definition 44. [Pep96, Pep04] Let $K$ be a theory of $\mathcal{L}$ and $\mathrm{S}$ a system of spheres centered on $[K]$. The multiple revision of $K$ by $\Gamma$ is:

$$
K \otimes A= \begin{cases}\cap f_{\mathrm{S}}(A) & \text { if }[A] \neq \varnothing \\ \mathcal{L} & \text { otherwise }\end{cases}
$$

However, Peppas observes that if $\mathrm{S}$ is restricted only by conditions (S1)-(S4), it is not secure to suppose that for a set of sentences $A$ there is always a smallest sphere intersecting $[A]$, even for consistent inputs. Then, not every system of spheres is sufficient to produce a multiple revision function. It makes the function $\otimes$ generated by $(\mathrm{S} \otimes)$ not well defined, which demands an extra constraint on S:

(SM) For every consistent set of sentences $A$ there exists a smallest sphere in $\mathrm{S}$ intersecting $[A]$.

Condition (SV) implies condition (SM) but not the converse.

(SM) alone is not enough for our purposes. We need a second condition that involves the idea of an elementary set of consistent complete theories. As defined in Section 2.6.6, a non-empty set $U$ of consistent complete theories is elementary iff $[\cap U]=U$. Nonetheless, not every set $U$ of consistent complete theories is elementary and, also, not every system of spheres $\mathrm{S}$ contains only elementary elements. The condition below establishes a requirement on the intersection of $[A]$ with $C_{\mathrm{S}}(A)$ :

(SD) For every nonempty $A \subseteq \mathcal{L}$, if there is a smallest sphere $C_{\mathrm{S}}(A)$ in $\mathrm{S}$ intersecting $[A]$, then $C_{\mathrm{S}}(A) \cap[A]$ is elementary.

Definition 45. [Pep96, Pep04] A system of spheres $\mathrm{S}$ is called a well-ranked system of spheres if it satisfies (SM) and (SD).

For studying multiple revision, the author restricted the systems of spheres considered to the family of well-ranked ones. He recalls the postulates for multiple revision as defined by Lindström[Lin91], calling a function $\otimes$ that obeys the set of postulates as a multiple revision function.

Theorem 26. [Pep04] Let $K$ be a theory of $\mathcal{L}$. If $\otimes$ is a function satisfying $(K \otimes 1)-(K \otimes 8)$, then there exists a well-ranked system of spheres $\boldsymbol{S}$ centred on $[K]$ such that for all nonempty $A \subseteq \mathcal{L}$, condition $(S \otimes)$ is satisfied for all $A \subseteq \mathcal{L}$. Conversely, if $\boldsymbol{S}$ is a well-ranked system of spheres centered on $[K]$, then the function $\otimes$ induced from $\mathrm{S}$ by means of $(S \otimes)$ satisfies the postulates $(K \otimes 1)-(K \otimes 8)$.

On the connection between multiple revision and AGM sentence revision, the author brings the definition of restriction and extendability:

Definition 46. [Pep96] For a multiple revision $\otimes$, the restriction of $\otimes$ to sentences is a function * defined such that, for all $K$ that are theories of $\mathcal{L}$ and $\varphi \in \mathcal{L}, K * \varphi=K \otimes\{\varphi\}$. An AGM revision function * is extendable iff there exists a multiple revision function $\otimes$ whose restriction to sentences is *.

Based on the results from [Lin91], Peppas states that the class of extendable revision functions corresponds to the family of revision functions corresponding, by means of $\left(\mathrm{S}^{*}\right)$, 
to well-ranked systems of spheres. As a consequence, all well behaved revision functions are extendable, given Lemma 3 and that (SV) implies (SM).

About the plausibility to reduce multiple revision to sentence revision, if the input is finite, the reduction is presented as already shown in the previous section, i.e., $K \otimes A=K *$ $\bigwedge A$. If, on the other hand, the input is infinite, a possibility of reduction is proposed in the form of a theorem that works for sets $A$ of arbitrary size. Nevertheless, it depends on a boundness condition. A multiple revision function $\otimes$ is bounded iff there exists a system of spheres $\mathrm{S}$ corresponding to $\otimes$ by means of $(\mathrm{S} \otimes)$ that has only finitely many spheres. Let $\mathcal{Z}(A)=\left\{\wedge C: C \subseteq_{f} A\right\}$. Then:

Theorem 27. [Pep96] Let $\otimes$ be a bounded multiple revision function and * its restriction to sentences. Then for any theory $K$ and any set of sentences $A$ of $\mathcal{L}$, the condition $(K \otimes F)$ holds as follows: $K \otimes A=\bigcap_{\varphi \in \mathcal{Z}(A)}(K * \varphi)+A$.

$(K \otimes F)$ defines a reduction that starts with a revision of $K$ by every finite conjunction $\varphi$ of sentences in $A$. Then, each such revised theory $K * \varphi$ is expanded by $A$ and, finally, all expanded theories $(K * \varphi)+A$ are intersected. A multiple revision function that can be reduced to sentence revision through $(K \otimes F)$ is called sentence-reducible at $K$.

Now consider the following condition related to a system of spheres S:

(SF) For every $G \subseteq \mathrm{S}, \bigcup G$ is elementary.

This is a stronger version of (SD) and is exactly the smoothness condition needed for the reduction of multiple revision to sentence revision in the spirit of $(K \otimes F)$ :

Theorem 28. [Pep04] Let $K$ be a theory of $\mathcal{L}, \otimes$ a multiple revision function and * its restriction to sentences. Moreover, let $\mathrm{S}$ be a well-ranked system of spheres centered on $[K]$ that corresponds to $\otimes$ by means of $(S \otimes)$. Then, $\mathrm{S}$ satisfies $(S F)$ iff $\otimes$ satisfies $(K \otimes F)$.

This theorem demonstrates that the multiple revision functions generated by wellranked systems of spheres satisfying (SF) are exactly those that are sentence-reducible at $K$. As a final consideration, it was observed in [DJ12] that this approach does not support empty inputs.

\subsubsection{Extra Constraints}

In [PKW12], the authors continued the issues addressed in [ZF01]. They observe that the limit postulate, while necessary, creates problems on other aspects. $(K \otimes L P)$ demanded additional constraints on systems of spheres and its relationship with the condition defined in Proposition 2 was still an open problem.

Theorem 29. [PKW12] There exists a consistent theory $K$ and a multiple revision function $\otimes$ satisfying $(K \otimes 1)-(K \otimes 8)$ such that $\otimes$ satisfies $(K \otimes L P)$ but violates $(K \otimes F)$ at $K$.

From the theorem above we can conclude that $(K \otimes L P)$ is strictly weaker than $(K \otimes$ $F)$.

Considering proper spheres, it is possible to add an extra restriction to them:

(EL) All proper spheres in S are elementary. 
However, even though it is intuitive, there is a dissimilarity between $(K \otimes L P)$ and (EL):

Lemma 4. [PKW12] There is a consistent theory $K$ and a well-ranked system of spheres $\mathrm{S}$ centered on $[K]$ such that $\mathrm{S}$ satisfies $(E L)$ and yet the multiple revision function $\otimes$ induced from $\mathrm{S}$ violates $(K \otimes L P)$.

In [PKW12] it is observed that this last result shows that (EL) is not enough to characterize $(K \otimes L P)$ and, at the same time, $(\mathrm{SF})$ is too strong. So there is a need for something in the middle. Before that, we need the definition of a finitely reachable sphere:

Definition 47. [PKW12] Let $K$ be a theory and $\mathrm{S}$ a system of spheres centered on $[K]$. A sphere $\mathcal{V}$ is finitely reachable in $\mathrm{S}$ iff there exists a consistent sentence $\varphi \in \mathcal{L}$ such that $C_{\mathrm{S}}(\varphi)=\mathcal{V}$.

Regard now the restrictions below on a system of spheres $\mathrm{S}$, where $\mathcal{V}$ is an arbitrary sphere in S:

(R1) If $\mathcal{V}$ is finitely reachable then $\mathcal{V}$ is elementary.

(R2) If $\mathcal{V}$ is finitely reachable then $\mathcal{V}^{c}$ is elementary.

(R3) If $\mathcal{V}^{c} \neq \mathcal{V}$ then $\left[\bigcap \mathcal{V}^{c}\right] \subseteq \mathcal{V}$.

These conditions represent the system-of-spheres counterpart of $(K \otimes L P)$ :

Theorem 30. [PKW12] Let $K$ be a theory, $\mathrm{S}$ a well-ranked system of spheres centered on $[K]$ and $\otimes$ the multiple revision function induced from $\mathrm{S}$ on $K$ via $(\mathrm{S} \otimes)$. Then $\otimes$ satisfies $(K \otimes L P)$ iff S satisfies $(R 1)-(R 3)$.

\subsubsection{Topological Approach}

The works presented in [ML14, ML15] use a topological approach in order to answer some open questions left by the study of multiple revision on systems of spheres.

Peppas demonstrated that a multiple revision operator generates an AGM operator when the new information is given by a proposition (which happens, for example, when the input set is finite and can be represented by the conjunction of its sentences). However, it is not clear if it is possible to extend an arbitrary AGM revision operator to a multiple revision operator and, if yes, how. Besides, even for a positive answer there is no certainty that this extension is unique. Meng an Li [ML14] stated that these topics are associated to an open problem pointed by Peppas: whether a multiple revision operator can be totally determined by its behavior on revisions by finite propositions.

Regarding the possibility to always extend an AGM operator to a multiple revision operator, Meng and Li gave a positive answer by demonstrating how to construct an extension of *. However, in relation to the uniqueness of the extension the answer is negative, proven through a counterexample.

As previously shown in this section, Peppas proved that it is possible to use well-ranked system of spheres to characterize multiple revision. Meng and Li [ML15] discussed the 
open problem on whether the condition (SD) is required to obtain a multiple revision operator. The result below shows that (SD) is a necessary condition:

Theorem 31. [ML15] Suppose $A$ is a nonempty subset of $\mathcal{M}_{\mathcal{L}}$ and $\mathrm{S}$ is a system of spheres on $A$ which satisfies (SM). If the corresponding revision operator satisfies $(K \otimes 1)-(K \otimes 8)$ via $(S \otimes)$, then $\mathrm{S}$ satisfies $(S D)$.

In the proof, it is shown that (SD) is a fundamental condition to guarantee the multiple revision operator satisfying $(K \otimes 7)$.

\subsection{Direct Constructions in Prioritized Revision}

In [Fuh88] the author, based on the Levi Identity, states that revision is clearly a compound operation, using this argument to defend that, given the not-very-complex nature of expansion operations, a theory of belief change should concentrate on the contraction operation. However, it would be interesting to study the definition of revision operations in a direct way, i.e., without using contraction as an intermediate step. This is one of the main goals of the works developed in [FKIRS12, VF16]. We also have the results from [TDG $\left.{ }^{+} 19\right]$, whose context involves information coming from several sources with different credibility values assigned to them. In all of the three articles the authors focused on working with belief bases.

\subsubsection{Multiple Package Revision}

In [FKIRS12], the authors defined some postulates for this direct operation. Let $B, A$ and $C$ be belief bases and ${ }^{*}$ be a binary package revision operator that receives a belief base and a set of sentences and returns a new belief base. So we have:

(inclusion) $B{ }^{*} p A \subseteq B \cup A$.

(success) $A \subseteq B{ }_{p} A$.

(weak success) If $A$ is consistent then $A \subseteq B{ }_{p} A$.

(relative success) $A \subseteq B *_{p} A$ or $B *_{p} A=B$.

(weak consistency) If $A$ is consistent then $B{ }^{*} p A$ is consistent.

(vacuity 1) If $A$ is inconsistent then $B *_{p} A=B$.

(vacuity 2) If $B \cup A H \perp$ then $B{ }_{p} A=B \cup A$.

(uniformity 1) Given two consistent sets $A$ and $C$, for all subsets $X$ of $B$, if $X \cup A \vdash$ $\perp$ iff $(X \cup C) \vdash \perp$, then $B \backslash\left(B *_{p} A\right)=B \backslash\left(B *_{p} C\right)$.

(uniformity 2) Given two consistent sets $A$ and $C$, for all subsets $X$ of $B$, if $X \cup A \vdash$ $\perp$ iff $(X \cup C) \vdash \perp$, then $B \cap\left(B *_{p} A\right)=B \cap\left(B *_{p} C\right)$.

(relevance) If $\alpha \in B \backslash\left(B{ }_{p} A\right)$ then there is a set $X$ such that $B{ }^{*} p \subseteq X \subseteq(B \cup A)$, $X$ is consistent with $A$ but $X \cup\{\alpha\}$ is inconsistent with $A$. 
(core-retainment) If $\alpha \in B \backslash\left(B{ }_{p} A\right)$ then there is a set $X$ such that $X \subseteq(B \cup A), X$ is consistent with $A$ but $X \cup\{\alpha\}$ is inconsistent with $A$.

Except for weak success and uniformity 1, the postulates above were adapted from similar postulates for singleton revision[Han99b].

By generalizing the techniques from classical belief base revision [Han99b], the authors defined two kinds of construction: Package Kernel Revision and Package Partial Meet Revision $^{14}$. In order to accomplish this, we first need the definition of two concepts: $A$ package-kernel and $A$-package-remainder. This last one was already given in Definition $33\left(B \downarrow_{p} A\right)$. For kernel we have:

Definition 48. [FKIRS12] Let $B, A$ be belief bases, where $A$ is consistent. The set of $A$ inconsistent-kernels of $B$, denoted by $B \downarrow_{p} A$, is the set of sets $X$ such that:

1. $X \subseteq B$;

2. $X \cup A$ is inconsistent;

3. For any $X^{\prime}$ such that $X^{\prime} \subset X \subseteq B$ then $X^{\prime} \cup A$ is consistent.

So, if you have a consistent set $A, B \downarrow_{p} A$ is the set of minimal $B$-subsets inconsistent with $A$.

After that, we also need the definitions of incision and selection functions. The selection function works in the same way as in Definition 24. The incision function is defined as below:

Definition 49. [FKIRS12] Let $B$ be a belief base. $\sigma$ is an incision function for $B$ iff, for all consistent sets $A$ :

1. $\sigma\left(B \downarrow_{p} A\right) \subseteq \bigcup B \downarrow_{p} A$

2. If $X \in B \downarrow_{p} A$ then $X \cap\left(\sigma\left(B \downarrow_{p} A\right)\right) \neq \varnothing$

Considering the definitions above, the domain for both functions is $\mathcal{P}(\mathcal{P}(\mathcal{L}))$, while the range for the incision function is $\mathcal{P}(\mathcal{L})$ and for the selection one is $\mathcal{P}(\mathcal{P}(L))$.

Having all these definitions, it is now possible to define the constructions and to relate them with the postulates.

Definition 50. [FKIRS12] Let $B$ be a belief base and $\sigma$ an incision function for $B$. The package kernel base revision on $B$ that is generated by $\sigma$ is the operator ${ }^{*} \sigma$ such that, for all sets $A$ :

$$
B *_{\sigma} A= \begin{cases}\left(B \backslash \sigma\left(B \Downarrow_{p} A\right)\right) \cup A & \text { if } \mathrm{A} \text { is consistent } \\ B & \text { otherwise }\end{cases}
$$

\footnotetext{
${ }^{14}$ In the original article, Package Revision is called Prioritized Change, in the sense that all the sentences from A should be present in the new belief base. Then, for the constructions, the names originally given were Prioritized Multiple Kernel Revision and Prioritized Multiple Partial Meet Revision.
} 
An operator * is a package kernel revision for $B$ iff there is an incision function $\sigma$ for $B$ such that for all sets $A, B * A=B *_{\sigma} A$.

Theorem 32. [FKIRS12] An operator * is a package kernel base revision for B iff it satisfies inclusion, weak consistency, weak success, vacuity 1, uniformity 1 (and uniformity 2), and core-retainment.

Definition 51. [FKIRS12] Let $B$ be a belief base and $\gamma$ a selection function for $B$. The package partial meet base revision on $B$ that is generated by $\gamma$ is the operator $*_{\gamma}$ such that, for all sets A:

$$
B *_{\gamma} A= \begin{cases}\bigcap_{B} \gamma\left(B \downarrow_{p} A\right) \cup A & \text { if } A \text { is consistent } \\ \text { otherwise }\end{cases}
$$

An operator * is a package partial meet revision on $B$ iff there is a selection function $\gamma$ for $B$ such that for all sets $A, B * A=B{ }_{\gamma} A$.

Considering the two definitions above, the domain and range for both functions ${ }^{*} \sigma$ and ${ }^{*}{ }_{\gamma}$ are $\mathcal{P}(\mathcal{L}) \times \mathcal{P}(\mathcal{L}) \rightarrow \mathcal{P}(\mathcal{L})$.

Theorem 33. [FKIRS12] An operator * is a package partial meet base revision for B iff it satisfies inclusion, weak consistency, weak success, vacuity 1, uniformity 2 (and uniformity 1), and relevance.

The only non-prioritized aspect of these operators is that no change is performed when the incoming belief base is inconsistent.

In a very similar way to what was done here, Valdez and Falappa [VF16] proposed, in the context of Horn Logic, two constructions for multiple package revision, one based on the Kernel approach and the other based on the Partial Meet one. Both of them were axiomatically characterized.

A Horn clause is a disjunction of literals (atoms or their negation) with at most one positive literal. A Horn formula is a conjunction of Horn clauses and a Horn theory is a set of Horn formulas. As examples of the importance of Horn logic, a large number of artificial intelligence systems are represented in Horn clause language and, at the same time, an extensive use of it has been observed in database theory (logic programming, truth maintenance systems and deductive databases).

Due to the weakened expressibility of Horn theories, there are some distinctions between classical AGM revision and Horn revision. Considering Horn theories, belief change operators are not (easily) inter-definable [ZPZ13] and, since some systems do not operate under classical logic, the AGM model cannot be always directly employed. That is why it is important to investigate suitable models to work with these specific situations, as done in [VF16].

\subsubsection{Multi-Source Multiple Revision}

In $\left[\mathrm{TDG}^{+} 19\right]$ we have a scenario of multiple revision derived from a collaborative ${ }^{15}$ multi-agent system, where a set of new beliefs come from different agents who have trust

${ }^{15}$ Collaborative means that the agents are honest and help each other. 
values associated to them and that are used to decide the previous beliefs that will be given up. The credibility assigned to each (informant) agent is represented by a strict partial order among them. This resource is applied to the incision function in order to orientate the incisions to be performed.

The authors combined and extended the approaches proposed in [FKIRS12], introducing a full axiomatic characterization of a multiple revision operator based on kernels. It is presumed that there is a finite set $\mathbb{A}=\left\{A, B, \ldots, Z, A_{1}, \ldots, Z_{n}\right\}$ of unique agent identifiers and all the informants have access to it. In order to associate each information in the belief base to its respective source, they use the epistemic model proposed in [TGFS12]. Then, the knowledge of an agent is represented by a set of information objects, which are pairs of the form (sentence, informant):

Definition 52. Given a propositional language $\mathcal{L}$ and a set $\mathbb{A}$ of agent identifiers, an information object is a tuple $(\alpha, X)$ where $\alpha \in \mathcal{L}$ and $X \in \mathbb{A}$ represents the information source for the sentence $\alpha$.

The belief base of an agent $A$ is denoted by $\mathcal{B}_{A}$ and taken as consistent. Given a belief base $\mathcal{B}_{A}, \operatorname{Exp}\left(\mathcal{B}_{A}\right)=\left\{\alpha \mid(\alpha, X) \in \mathcal{B}_{A}\right\}$ and $\operatorname{Ag}\left(\mathcal{B}_{A}\right)=\left\{X \mid(\alpha, X) \in \mathcal{B}_{A}\right\}$. A belief base $\mathcal{B}_{A}$ is considered consistent if $\operatorname{Exp}\left(\mathcal{B}_{A}\right)$ is consistent. Another interesting property is that a belief base could contain many information objects concerning the same sentence but coming from different agent identifiers, not configuring a case of redundancy.

The new information received by an agent is modeled as a set of information objects as well, considered to be consistent in itself. Besides, it is also supposed that the agent carrying out the revision operation has its own strict partial order ${ }^{16}$ that represents its concept of the ordering of the agents of the system. It is denoted by $<_{C O}^{X}$, where $X$ represents the agent identifier $X \in \mathbb{A}$ that owns this order. As an example, $A<_{C O}^{X} B$ represents that, for agent $X$, agent $A$ is less credible than $B$ (and, consequently, $B$ is more credible than $A$ ).

Before defining what a safe element is, it is necessary to remember from [AM85] the concept of hierarchy. A relation < over a set of propositions $S$ is a hierarchy iff it satisfies the non-circularity condition: for no $\alpha_{1}, \alpha_{2}, \ldots, \alpha_{n} \in S$ with $n \geq 1$ it holds that $\alpha_{1}<\alpha_{2}<\ldots<\alpha_{n}<\alpha_{1}$. For our purposes, we will consider hierarchies which are also transitive.

Definition 53. Let $\mathcal{B}_{A}$ be a knowledge base, $\mathcal{I}$ a consistent set of information objects to be added consistently to $\mathcal{B}_{A}$ and $<$ be an arbitrary transitive hierarchy over $\mathcal{B}_{A}$. An element $\alpha \in\left\{\mathcal{B}_{A} \cup \mathcal{I}\right\}$ is safe with respect to the revision of $\mathcal{B}_{A}$ by $\mathcal{I}$ iff $\alpha$ is not a minimal element (under $<$ ) of any minimal subset (under set inclusion) $\mathcal{B}^{\prime}$ of $\mathcal{B}_{A}$ such that $\mathcal{B}^{\prime} \cup \mathcal{I}$ is inconsistent.

\subsubsection{Postulates and Construction}

Let $\mathcal{B}, \mathcal{X}, \mathcal{I}$ and $\mathcal{I}^{\prime}$ be consistent belief bases, and * be a binary multiple belief revision operator. The authors proposed five postulates. Three of them (Inclusion, Weak Success and Uniformity) were borrowed from [FKIRS12]. The other two are:

(consistency) $\mathcal{B} * \mathcal{I}$ is consistent.

\footnotetext{
${ }^{16}$ Irreflexive, asymmetric and transitive binary relation.
} 
(safe retainment) $\alpha \in \mathcal{B} * \mathcal{I}$ iff $\alpha$ is a safe element with respect to $\mathcal{I}$ in $\mathcal{B}$.

For the construction, the concept of $\mathcal{I}$-inconsistent kernels $\left(\mathcal{B} \downarrow_{p} \mathcal{I}\right)$ is the same as in [FKIRS12] (Definition 48). It is important to observe that no element of $\mathcal{I}$ is part of the kernel set:

Observation 9. [TDG ${ }^{+}$19] Let $\mathcal{B}$ be a belief base and $\mathcal{I}$ be a consistent set of information objects. Suppose that $(\alpha, X) \in \mathcal{B}$ and $(\alpha, X) \in \mathcal{B}$. Then we have that $\alpha \notin \bigcup\left(\mathcal{B} \downarrow_{p} \mathcal{I}\right)$ and, therefore, $\mathcal{I} \cap \bigcup\left(\mathcal{B} \downarrow_{p} \mathcal{I}\right)=\varnothing$.

In the next step, there should be an incision function that chooses the less reliable information object in each inconsistent-kernel. In order to specify what is understood as "less reliable" in this context, we need a particular definition of safe element:

Definition 54. [TDG 19$]$ An information object $(\alpha, B)$ of $\mathcal{B}_{A}$ is safe with respect to revision by $\mathcal{I}$ in $\mathcal{B}_{A}$ iff $(\alpha, B) \notin \mathcal{X}, \forall \mathcal{X} \in \mathcal{B}_{A} \downarrow_{p} \mathcal{I}$ (i.e., there is no minimal subset $\mathcal{B}_{A}$ inconsistent with $\mathcal{I}$ that contains $(\alpha, B))$ or, if $\exists \mathcal{X} \in \mathcal{B} \downarrow_{p} \mathcal{I}$ such that $(\alpha, B) \in \mathcal{X}$, then $\exists(\beta, C) \in \mathcal{X}$ such that $C<_{C O}^{A} B$.

Now, it is possible to define an incision function:

Definition 55. [TDG ${ }^{+}$19] Let $\mathcal{B}$ be a belief base. Then $\sigma$ is a multi-source incision function for $\mathcal{B}$ iff for all consistent set of information objects $\mathcal{I}$ the three following conditions hold:

1. $\sigma\left(\mathcal{B} \downarrow_{p} \mathcal{I}\right) \subseteq \bigcup \mathcal{B} \downarrow_{p} \mathcal{I}$,

2. If $\mathcal{X} \in \mathcal{B} \downarrow_{p} \mathcal{I}$ then $\mathcal{X} \cap(\sigma(\mathcal{B} \downarrow \mathcal{I})) \neq \varnothing$,

3. $(\alpha, C) \in \sigma\left(\mathcal{B} \downarrow_{p} \mathcal{I}\right)$ iff for some $\mathcal{X} \in \mathcal{B} \downarrow_{p} \mathcal{I}$ such that $(\alpha, C) \in \mathcal{X}$, there does not exist $(\beta, B) \in \mathcal{X}$ where $B<_{C O}^{A} C$.

With all these definitions at hand, we can have the operator and a representation result:

Definition 56. [TDG $\left.{ }^{+} 19\right]$ Let $\mathcal{B}$ be a belief base, $\mathcal{I}$ a consistent set of information objects, and $\sigma$ be a multi-source incision function for $\mathcal{B}$. The multi-source kernel revision on $\mathcal{B}$ that is generated by $\sigma$ is the operator ${ }^{*}{ }_{\sigma}$ such that for all set $\mathcal{I}$ the following condition holds: $\mathcal{B} *_{\sigma} \mathcal{I}=\left(\mathcal{B} \backslash \sigma\left(\mathcal{B} \downarrow_{p} \mathcal{I}\right)\right) \cup \mathcal{I}$

Theorem 34. [TDG $\left.{ }^{+} 19\right]$ An operator $*_{\sigma}$ is a multi-source multiple kernel revision for $\mathcal{B}$ iff it satisfies the postulates of inclusion, weak success, consistency, uniformity and saferetainment.

Since this approach was developed for multi-agent contexts with several informants and a credibility relation among them, if there is only one agent or if it is impossible to compare the agents we reach an extreme case:

Proposition 3. [TDG 19$]$ Let $\mathcal{B}_{A}$ be a belief base, ${ }_{\sigma}{ }_{\sigma}$ be a multi-source multiple kernel revision, $\mathcal{I}$ be an input set and $<_{C O}^{A}$ be a credibility order. If $A g\left(\mathcal{B}_{A}\right)$ is a singleton set or $<_{C O}^{A}=\varnothing$ then $\mathcal{B}_{A} *_{\sigma} \mathcal{I}=\left(\mathcal{B}_{A} \backslash \bigcup \mathcal{B}_{A} \Downarrow_{p} \mathcal{I}\right) \cup \mathcal{I}$.

This drastic result is comparable to Full Meet Revision [Han99b]. 


\subsubsection{Comparing to Other Approaches}

Most of the revision approaches using incision functions, whether singleton or multiple, are of a general prospect, given that there is no specification of how to choose elements of a kernel. On the other hand, the operation proposed in [TDG $\left.{ }^{+} 19\right]$ applies the credibility order associated to the different agents as a way to determine the incisions.

This idea that an specific sentence is preferred as an option for removal over others is not new. In the Epistemic Entrenchment approach[GM88, Gär88, MLH00], there is an epistemic entrenchment ordering $\varsigma_{E E}$ over the sentences of a belief set that is used to determine the revision process. The point is that the formulas kept in the belief set during revision are the ones that are more entrenched. Nonetheless, while in [TDG $\left.{ }^{+} 19\right]$ the authors work with belief bases, multiple agents and multiple sentences as input, Epistemic Entrenchment works with belief sets, revision by single sentences and no identification of sources.

\subsection{Final Remarks}

The approaches presented in this chapter involve all of the three epistemic models referred in the Introduction: belief sets, belief bases and possible worlds. While in [Fuh88, Zha96, ZCZC97, ZF01] the focus was on belief sets, in [Han93, FKIRS12, VF16, TDG ${ }^{+}$19] they worked with belief bases. Still, the authors in [Lin91, Pep96, Pep04, PKW12, ML14, ML15] dealt with possible worlds.

Most of the authors developed their theories using classical propositional logic, except for Lindström [Lin91] (propositional deductive logic), Zhang et. al. [Zha96, ZCZC97, ZF01] (classical first-order logic) and Valdez and Falappa [VF16] (Horn logic). The works in [Pep96, Pep04, PKW12] do not refer an specific logic but make some assumptions about the language and its associated consequence operator that are compatible with the AGM assumptions (see Section 2.6.2). Regarding the works from Dongmo Zhang et. al., it is important to remark that, although the authors claim to have extended AGM theory to first-order logic, they also say that there are no postulates designed to deal with quantifiers or predicates in the result, leaving further explorations of first-order properties of belief revision for future work.

In relation to the finiteness of the input, the works that considered the possibility for infinite sets were [Lin91, Zha96, ZCZC97, ZF01, Pep96, Pep04, PKW12, ML14, ML15].

Regarding constructions, in [Fuh88, Han93, Fuh97, ZCZC97] we have partial meet, while in [FKIRS12, VF16] we have partial meet and kernel and, in [TDG ${ }^{+}$19], kernel. The authors in [Lin91, Pep96, Pep04, PKW12, ML14, ML15] worked with system of spheres and, in [Zha96, ZF01], nicely-ordered partition. Regarding postulates, it would be possible to divide them in two great groups. In [Fuh88, Lin91, Zha96, Pep96, Fuh97, ZCZC97, ZF01, Pep04, PKW12, ML14, ML15] they use the same set of postulates (as proposed by Fuhrmann and adapted by Lindström), focusing on belief sets. On the other hand, in [Han93, FKIRS12, VF16, TDG ${ }^{+}$19] the set of postulates is smaller and closer to belief bases.

Table 3.1 summarizes the main characteristics of the different approaches analyzed 
in this chapter regarding the epistemic model used, the underlying logic considered, the validity of the results for any set or not and possible given constructions. Still, some observations need to be made. Regarding the assumption on the finiteness or not of the input, when the work is not specifically clear about [FKIRS12, VF16, TDG ${ }^{+} 19$ ], we preferred to consider them as for finite inputs.

\subsection{Open Problems}

As a final analysis, it is possible to list some open problems. Regarding the operators defined in [FKIRS12], there is the possibility to study the interrelations between the package kernel and partial meet revision operators. In the topological approach for system of spheres ([ML14]), it is pointed as an open question if there is an irreducible (or smallest) well-ranked system of spheres for a given multiple revision operator.

In relation to other approaches, Horn multiple revision [VF16] could be studied in a selective approach (partial acceptance of the input). Regarding the underlying logic of each model presented here, since most of them were developed for propositional logic, an important future work is to investigate how they can be adapted to work with other logics whose restrictions are different from classical logic. As an example, Multi-source Multiple Revision [TDG ${ }^{+} 19$ ] could be combined with the results from [KPHS07] in order to implement a multi-source multiple revision approach for DL ontologies, in which the investigation for conflicts is conducted applying the methods introduced by Kalyanpur et al. Still, in [TDG $\left.{ }^{+} 19\right]$ there is an assumption about the consistency of the input; this assumption could be abandoned in order to study distinct behaviors when there are conflicts in the epistemic input. 


\begin{tabular}{|c|c|c|c|c|c|c|c|}
\hline \multirow{2}{*}{ Paper } & \multicolumn{3}{|c|}{ Epistemic Model } & \multirow{2}{*}{ Logic } & \multicolumn{2}{|c|}{ Type of Input } & \multirow{2}{*}{ Construction } \\
\hline & B. Set & B. Base & Poss. Worlds & & Finite & Infinite & \\
\hline [Fuh88] & $\bar{X}$ & & & Class. Propositional & & $\overline{\mathrm{X}}$ & Partial Meet \\
\hline [Lin91] & & & $\mathrm{X}$ & Proposit. Deductive & & $\mathrm{X}$ & System of Spheres \\
\hline [Han93] & & $\mathrm{X}$ & & Class. Propositional & $\mathrm{X}$ & & Partial Meet \\
\hline [Zha96, ZF01] & $\mathrm{X}$ & & & Class. First-Order & & $\mathrm{X}$ & Nicely-Ordered Part. \\
\hline [Pep96, Pep04] & & & $\mathrm{X}$ & AGM Assumptions & & $\bar{X}$ & System of Spheres \\
\hline [Fuh97] & $\mathrm{X}$ & & & Class. Propositional & & $\mathrm{X}$ & Partial Meet \\
\hline [ZCZC97] & $\mathrm{X}$ & & & Class. First-Order & & $\mathrm{X}$ & Partial Meet \\
\hline [PKW12] & & & $\mathrm{X}$ & AGM Assumptions & & $\mathrm{X}$ & System of Spheres \\
\hline [FKIRS12] & & $\mathrm{X}$ & & Class. Propositional & $\mathrm{X}$ & & P. Meet and Kernel \\
\hline [ML14] & & & $\mathrm{X}$ & Class. Propositional & & $\mathrm{X}$ & System of Spheres \\
\hline [ML15] & & & $\mathrm{X}$ & Class. Propositional & & $\mathrm{X}$ & Total Pre-Order on Worlds \\
\hline [VF16] & & $\mathrm{X}$ & & Horn & $\mathrm{X}$ & & P. Meet and Kernel \\
\hline$\left[\mathrm{TDG}^{+} 19\right]$ & & $\mathrm{X}$ & & Class. Propositional & $\mathrm{X}$ & & Kernel \\
\hline
\end{tabular}

Table 3.1: Summary of the general characteristics of the different prioritized approaches. 



\section{Chapter 4}

\section{Non-Prioritized Multiple Revision}

As stated in Section 3.1.2, the multiple revision operation can happen in two main ways. While in the previous chapter we brought different approaches for prioritized multiple revision, in the present one we are going to explore some models for the non-prioritized kind, which means that the incoming beliefs do not have total priority in relation to the initial ones: the agent can incorporate only a part of the new beliefs whilst refusing the other part.

In the first three sections we present some works on choice revision ([Zha18, Zha19, Han17]). In [Han17] we have the introduction of a construction of choice revision through the descriptor revision framework, while its formal properties were studied in [Zha19]. Another modelling based on multiple believability relations was also explored in [Zha19] as a possible definition of choice revision.

In Section 4.4 we also have the definition of some multiple revision operators that work in a non-prioritized way but based on explanations ([FKIS02]), being an average model between semi-revision [Han97] and merge [Fuh97]. In Section 4.5 the chosen approach is a generalization of Selective Revision $\left(\left[\mathrm{KTF}^{+} 11\right]\right)$ but based on Deductive Argumentation [BH01].

In Section 4.6 the case is explored when the input set is inconsistent but may has consistent individual pieces of knowledge ([Del11]). Finally, Section 4.7 shows two works that deal with the context in which the agent has core beliefs, i.e., some pieces of knowledge that are immune to revision ([YJW14],[Yua17]).

\subsection{The Levi Identity Model}

Zhang [Zha18] proposed two types of choice revision, one on the contraction + expansion approach and the other on the expansion + contraction one. Both of them were developed for belief bases and were axiomatically characterized. Before defining the operations, the author introduced an auxiliary operation called Partial Expansion, which 
is a generalization of the traditional expansion operation. This generalization uses the two resources defined below. The first one is the partial sum set $(\bowtie)$ between two sets $B$ and $A$. The second one is a kind of selection function over the partial sum set.

Definition 57. [Zha18] Given two sets of sentences $B$ and $A$, let $B \bowtie A$ be the Partial Sum Set between them. $X \in B \bowtie A$ iff:

- $X \subseteq(B \cup A)$;

- $B \subseteq X$;

- $X \cap A \neq \varnothing$.

The partial sum set of $B$ by $A$ is a set of sets formed by all possible expansions of $B$ by parts of $A$, given that each element of $B \bowtie A$ contains the whole $B$ with some part of A.

The selection function brought by the author is slightly different from the one given in Definition 24. Actually, the difference is in its signature, which makes it more general:

Definition 58. [Zha18] A selection function $\gamma$ is any function $\gamma: \mathcal{P}(L) \times \mathcal{P}(\mathcal{P}(L)) \rightarrow$ $\mathcal{P}(\mathcal{P}(L))$ such that:

1. If $\mathbb{Y} \neq \varnothing$ then $\varnothing \neq \gamma(X, \mathbb{Y}) \subseteq \mathbb{Y}$;

2. If $\mathbb{Y}=\varnothing$ then $\gamma(X, \mathbb{Y})=\{X\}$.

Definition 59. [Zha18] Let $\gamma$ be a selection function. $\gamma$ is $\bowtie$-consistency-preserving iff for all $B$ and $A$ : if there exists $a$ set $X \in B \bowtie A$ such that $X \forall \perp$, then $\bigcup \gamma(B, B \bowtie A) \forall \perp$. tion:

With these resources at hand, it is possible to define the Partial Expansion opera-

Definition 60. [Zha18] An operator $+(\mathcal{P}(L) \times \mathcal{P}(L) \rightarrow \mathcal{P}(L))$ is a partial expansion iff there exists a selection function $\gamma$ such that for all sets $B$ and $A$,

$$
B+A=\bigcup \gamma(B, B \bowtie A) \text {. }
$$

+ is choice-consistency-preserving iff $\gamma$ is $\bowtie$-consistency-preserving.

Therefore, the default expansion operation and its generalized version for sets referred in Chapters 2 and 3, respectively, are particular cases of partial expansion.

As an example, let $B=\{\alpha, \beta, \varphi\}$ and $A=\{\neg \varphi, \delta\}$. According to Definition 57, $B \bowtie$ $A=\{\{\alpha, \beta, \varphi, \delta\},\{\alpha, \beta, \varphi, \neg \varphi\},\{\alpha, \beta, \varphi, \neg \varphi, \delta\}\}$. So we can have seven different selection functions: one for each possible combination in selecting one or more elements of $B \bowtie A$. However, according to Definition 59, the only consistency-preserving selection function is the one that selects only $\{\alpha, \beta, \varphi, \delta\}$. Let $\gamma_{1}$ be this selection function. The partial expansion of $B$ by $A$ applying $\gamma_{1}$ is $B+A=\bigcup \gamma_{1}(B, B \bowtie A)=\{\alpha, \beta, \varphi, \delta\}$.

The following representation result was given:

Theorem 35. [Zha18]

1. + is a partial expansion iff it satisfies the following postulates: for every sets $B, A$ and $C$, 
4.1 | THE LEVI IDENTITY MODEL

$$
\begin{aligned}
& \text { (+-inclusion) } B+A \subseteq B \cup A \\
& \text { (+-preservation) } B \subseteq B+A \\
& \text { (+-success) If } A \neq \varnothing, \text { then } A \cap(B+A) \neq \varnothing \\
& \text { (+-coincidence) If } B \cap A \neq \varnothing \text { and } A \subseteq C \subseteq(B \cup A) \text {, then } B+A=B+C
\end{aligned}
$$

2. + is consistency-preserving iff it in addition satisfies:

(+-consistency) If there exists $X$ such that $B \subseteq X \subseteq(B \cup A)$ and $X \forall \perp$, then $B+A \forall \perp$

Lemma 5. [Zha18] Let + be a partial expansion operator operator. + satisfies $\dot{+}$-coincidence iff it satisfies:

(+-Coincidence) If $B \cap A \neq \varnothing, B \cap C \neq \varnothing$ and $B \cup A=B \cup C$, then $B+A=B+C$.

In order to proceed with the definition of Choice Revision, the author adapted a definition of negation set given in [Han92]:

Definition 61. [Zha18] Let $A$ be some set of sentences. Then the negation set of $A$, denoted by $n e g(A)$, is defined as follows:

1. $\operatorname{neg}(\varnothing)=\mathrm{\top}$,

2. $\operatorname{neg}(A)=\cup_{n \geq 1}\left\{\neg \alpha_{1} \vee \neg \alpha_{2} \vee \cdots \vee \neg \alpha_{n} \mid \alpha_{i} \in A\right.$ for every $i$ such that $\left.1 \leq i \leq n\right\}$

The difference between this definition and the one given by Hansson (Definition 29) is that the original one defines $n e g(\varnothing)=\perp$. However, Zhang states that, when $A=\varnothing$, it is intuitive that $B *_{c} A=B$, which would be readily achievable if $n e g(\varnothing)$ is defined as T.

Finally, two constructions are shown: one for internal revision and one for external revision, both of them depending on contraction.

\subsubsection{Internal Choice Revision}

The operator is defined in the following way:

Definition 62. [Zha18] An operator ${ }^{*}(\mathcal{P}(L) \times \mathcal{P}(L) \rightarrow \mathcal{P}(L))$ is an internal choice base revision iff there exists a choice contraction $-_{c}$ and a consistency-preserving partial expansion + such that for all sets $B$ and $A$,

$$
B *_{c} A=B-{ }_{c} \operatorname{neg}(A)+A .
$$

A representation result was shown:

Theorem 36. [Zha18] (Representation theorem for internal choice revision)

1. ${ }^{*}$ is an internal choice base revision on consistent belief bases with finite inputs iff it satisfies the following conditions: for every consistent $B$ and finite $A$ and $C$,

(inclusion) $B *_{c} A \subseteq(B \cup A)$

(success) If $A \neq \varnothing$, then $A \cap\left(B *_{c} A\right) \neq \varnothing$ 
(iteration) $B *_{c} A=\left(B \cap\left(B *_{c} A\right)\right) *_{c} A$

(weak consistency) If $A \not \equiv\{\perp\}$, then $B *_{c} A H \perp$

(coincidence) If $A \cap B \neq \varnothing$ and $A \subseteq C \subseteq(A \cup B)$, then $B *_{c} A=B *_{c} C$

(uniformity) If it holds for all $B^{\prime} \subseteq B$ that $B^{\prime} \cup\{\varphi\} \vdash \perp$ for some $\varphi \in A$ iff $B^{\prime} \cup\{\psi\} \vdash \perp$ for some $\psi \in C$, then $B \cap\left(B *_{c} A\right)=B \cap\left(B *_{c} C\right)$

(relevance) If $\varphi \in B \backslash B *_{c} A$, then there is some $B^{\prime}$ with $B \cap\left(B *_{c} A\right) \subseteq B^{\prime} \subseteq B$, such that $B^{\prime} \cup\{\psi\} \forall \perp$ for some $\psi \in A$ and $B^{\prime} \cup\{\varphi\} \cup\{\lambda\} \vdash \perp$ for every $\lambda \in A$

2. ${ }^{*}$ is additionally unified iff it satisfies in addition the following:

(redundancy) If $B \cup Z \forall \perp, A \neq\{\perp\}, A \neq \varnothing$ and it holds for every $\varphi \in Z$ that $\varphi \vdash \neg \psi$ for all $\psi \in A$, then $B *_{c} A=(B \cup Z) *_{c} A$

Although in Definition 29 we have a simplification of $n e g(A)$ when $A$ is finite (being possible to represent it in one single sentence), it is not suitable to use it here. Given that the first step of the operator is a (choice) contraction, the contraction by the sentential negation of $A$ would retract from $B$ more than necessary, since it would make $B$ compatible with the whole $A$. Therefore, for this context it is more adequate to have $n e g(A)$ as a set.

\subsubsection{External Choice Revision}

The operator is defined in the following way:

Definition 63. [Zha18] An operator $*_{c}(\mathcal{P}(L) \times \mathcal{P}(L) \rightarrow \mathcal{P}(L))$ is an external choice base revision iff there exists a package contraction $-_{p}$ and a partial expansion + such that for all $B$ and $A$,

$$
B{ }_{c} A=B+A{ }_{p} n e g\left(A^{\prime}\right)
$$

where $A^{\prime}=(B+A) \backslash B$.

A representation result was shown:

Theorem 37. [Zha18] (Representation theorem for external choice revision)

1. ${ }^{*}$ is an external choice base revision iff, for all $B, B_{1}, B_{2}, A$ and $C$, it satisfies inclusion, success, coincidence and:

(confirmation) If $\left(A \cap\left(B *_{c} A\right)\right) \subseteq B$, then $B{ }^{*}{ }_{c} A=B$

(weak consistency) If $\left(B *_{c} A\right) \backslash B \neq \varnothing$ and $\left(B *_{c} A\right) \backslash B H \perp$, then $B *_{c} A H \perp$

(uniformity) If $B_{1} \neq\left(\left(B_{1} *_{c} A\right) \cup B_{1}\right)=B=\left(\left(B_{2}{ }^{*} c\right) \cup B_{2}\right) \neq B_{2}$ and it holds for all $B^{\prime} \subseteq B$ that $B^{\prime} \cup\left(\left(B_{1} *_{c} A\right) \backslash B_{1}\right) \forall \perp$ iff $B^{\prime} \cup\left(\left(B_{2} *_{c} C\right) \backslash B_{2}\right) \forall \perp$, then $B_{1} *_{c} A=B_{2} *_{c} C$

(relevance) If $\varphi \in B \backslash B{ }^{*} A$, then there is some $B^{\prime}$ with $B{ }^{*}{ }_{c} A \subseteq B^{\prime} \subseteq B \cup\left(B{ }^{*}{ }_{c} A\right)$, such that $B^{\prime} \forall \perp$ and $B^{\prime} \cup\{\varphi\} \vdash \perp$

2. $*_{c}$ is additionally unified iff it satisfies in addition the following: 
(strong Uniformity) If it holds for all $X \subseteq \mathcal{L}$ that $X \subseteq B_{1} \cup\left(B_{1} *_{c} A\right)$ and $X \cup\left(\left(B_{1}{ }^{*} c A\right) \backslash B_{1}\right)$ is consistent iff $X \subseteq B_{2} \cup\left(B_{2} C\right)$ and $X \cup\left(\left(B_{2} *_{c} C\right) \backslash B_{2}\right)$ is consistent, then $B_{1}{ }^{*} A=B_{2}{ }_{c} C$.

\subsubsection{Common and Distinct Aspects}

Due to the usage of set-negation to perform the contraction part of the choice operation, these approaches proposed in [Zha18] depends on negation and on disjunction of sentences.

However, the kind of contraction used in each one is different. Internal choice revision applies choice contraction by the negation set of the input because the purpose is to make the belief base consistent with at least a subset of the input. On the other hand, external choice revision first applies a partial expansion of the base by the input set; that is why a package contraction by $n e g\left(A^{\prime}\right)$ is necessary: in order to make $B+A$ consistency with the whole $A^{\prime}$.

\subsubsection{Comparing with Selective Revision}

[FKIRS12] and [Zha18] remark that the choice revision operation is not equal to Selective Revision [FH99]. Although this last one is of non-prioritized sort as well, it is for single sentences in the input and, in addition, that first one is not reducible to the last. Still about reduction, it is observed in [Spo10] that when we need to accept at least one of the beliefs of an input set there is no evident answer. It is not the same as accepting the disjunction of the input sentences because you may admit their disjunction without admitting any of them, which means that it is not a solution.

\subsection{The Descriptor Revision Approach}

In [Zha19], the author explores further a proposal of choice revision based on another approach to belief change named Descriptor Revision[Han14a]. This approach applies a "select-direct" procedure by considering that there is a set of belief sets that work as possible results of belief change and this change is implemented through a direct choice among these possible results.

Both of the types proposed were axiomatically characterized through a set of postulates and a representation theorem, with the assumption of a finite language.

It is important to observe that, in this approach, revision was explored without taking into account its connection with contraction, i.e., it was defined without using contraction as an intermediate step.

\subsubsection{Descriptor Revision}

Before bringing the constructions and properties of choice revision in this context, we show some background about the descriptor revision framework, based on [Han14a]. 
Belief descriptors are a flexible construction for describing belief sets. Let $\mathfrak{B}$ be a metalinguistic belief predicate. In a belief change context, we need to represent not just belief sets but input sentences as well. So $\mathfrak{B}$ takes sentences in the object language as arguments. For any sentence $\alpha \in \mathcal{L}, \mathfrak{B}_{\alpha}$ means that $\alpha$ is believed in that belief set. It is important to remark that $\mathfrak{B}$ is not considered an element of the object language. More formally:

Definition 64. [Han14a] Let $\mathfrak{B}$ be a metalinguistic belief predicate and $\alpha$ a sentence in the object language $\mathcal{L}$. An atomic belief descriptor is a sentence $\mathfrak{B}_{\alpha}$. It is satisfied by a belief set $K$ iff $\alpha \in K$.

A molecular belief descriptor (denoted by lowercase Latin letters) is a truth-functional combination of atomic descriptors. Conditions of satisfaction are defined inductively, such that $K$ satisfies $\neg p$ iff it does not satisfy $p$, it satisfies $p \vee q$ iff it satisfies either $p$ or $q$, etc.

A composite belief descriptor (in short: descriptor; denoted by uppercase Greek letters) is a non-empty set of molecular descriptors. A belief set $K$ satisfies a composite descriptor $\Psi$ iff it satisfies all its elements.

A descriptor is satisfiable within a set of belief sets iff it is satisfied by at least one of its elements.

By this definition, descriptors are an appropriate form to represent the success condition of different kinds of belief change operations. For multiple revision, there are two possibilities. Revision by a set $\left\{\alpha_{1}, \ldots, \alpha_{n}\right\}$ has the success condition $\left\{\mathfrak{B}_{\alpha_{1}}, \ldots, \mathfrak{B}_{\alpha_{n}}\right\}$ for package revision and $\left\{\mathfrak{B}_{\alpha_{1}} \vee \ldots \vee \mathfrak{B}_{\alpha_{n}}\right\}$ for choice revision.

Descriptor revision, denoted by $\circ$, is an operation of belief change that goes from a belief set $K$ to a new belief set $K \circ \Psi$ where $\Psi$ is a descriptor representing the success condition. This way, other operations of belief change already defined become special cases of descriptor revision. Many constructions for descriptor revision operations were introduced in [Han14a]. Before bringing the relational model of interest, a relation needs to be defined. Given a set of belief sets $\mathbb{X}$, was defined as a relation on $\mathbb{X}$ called belief set ordering.

Definition 65. [Han14a] Let $\mathbb{X}$ be a set of belief sets, $\leqq$ be a relation on $\mathbb{X}$, and $\mathbb{Y} \subseteq \mathbb{X}$. Then $X$ is $\leqq$-minimal in $\mathbb{Y}$ iff $X \in \mathbb{Y}$ and $X \leqq Y$ for all $Y \in \mathbb{Y}$.

Now, the relational model:

Definition 66. [Han14a] $]^{1}(\mathbb{X}, \leqq)$ is a relational select-direct model (in short: relational model) with respect to $K$ iff it satisfies:

$(\mathbb{X} 1) \mathbb{X}$ is a set of belief sets.

$(\mathbb{X} 2) K \in \mathbb{X}$.

$(\leqq 1) K \leqq X$ for every $X \in \mathbb{X}$.

(ミ2) For any descriptor $\Psi$, if $\{X \in \mathbb{X} \mid \Psi \subseteq C n(X)\}$ (denoted by $\mathbb{X}^{\Psi}$ ) is not empty, then it has a unique $\leqq-m i n i m a l ~ e l e m e n t ~ d e n o t e d ~ b y ~ \mathbb{X}_{<}^{\Psi}$.

\footnotetext{
${ }^{1}$ This definition is more general than the original one, as shown in [Zha17].
} 
A descriptor revision $\circ$ on $K$ is based on (or determined by) some relational model $(\mathbb{X}, \leqq)$ with respect to $K$ iff for any descriptor $\Psi$ :

$$
\langle\leqq t o \circ\rangle \quad K \circ \Psi= \begin{cases}\mathbb{X}_{<}^{\Psi} & \text { if } \mathbb{X}_{<}^{\Psi} \text { is not empty } \\ K & \text { otherwise }\end{cases}
$$

$\mathbb{X}$ is the outcome set that contains all the potential results under several belief change patterns. The ordering $\leqq$ displays a direct-selection mechanism, which chooses the final result among candidates that satisfy an explicit success condition.

A descriptor revision built in this way is called a relational descriptor revision.

\subsubsection{Choice Revision from Descriptor Revision}

For a choice revision ${ }^{*} c$ with a finite input, the success condition could be represented by descriptor $\left\{\mathfrak{B}_{\alpha_{1}} \vee \ldots \vee \mathfrak{B}_{\alpha_{n}}\right\}$. Hansson constructed choice revision through descriptor revision this way:

Definition 67. [Han17] Let $\circ$ be some descriptor revision. A choice revision $*_{c}$ on $K$ is based on (or determined by) $\circ$ iff for any finite set $A$ :

$$
\left\langle\circ \text { to } *_{c}\right\rangle \quad K *_{c} A= \begin{cases}K \circ\left\{\mathfrak{B}_{\alpha_{1}} \vee \ldots \vee \mathfrak{B}_{\alpha_{n}}\right\} & \text { if } A=\left\{\alpha_{0}, \ldots, \alpha_{n}\right\} \neq \varnothing \\ K & \text { otherwise }\end{cases}
$$

The observations below establishes some properties to be satisfied by the operation:

Observation 10. [Zha19] Let ${ }_{c}$ be a choice revision determined by any relational descriptor revision $(\mathbb{X}, \leqq)$. Then it satisfies the following properties:

$\left(*_{c} 1\right) C n\left(K *_{c} A\right)=K *_{c} A\left(*_{c}\right.$-closure $)$

$\left(*_{c} 2\right) K *_{c} A=K$ or $A \cap\left(K *_{c} A\right) \neq \varnothing\left(*_{c}\right.$-relative success $)$

$\left(*_{c} 3\right)$ If $A \cap\left(K *_{c} B\right) \neq \varnothing$, then $A \cap\left(K *_{c} A\right) \neq \varnothing\left(*_{c}\right.$-regularity $)$

$\left(*_{c} 4\right)$ If $A \cap K \neq \varnothing$, then $K *_{c} A=K\left(*_{c}\right.$-confirmation)

$\left(*_{c} 5\right)$ If $\left(K *_{c} A\right) \cap B \neq \varnothing$ and $\left(K *_{c} B\right) \cap A \neq \varnothing$, then $K *_{c} A=K *_{c} B\left(*_{c}\right.$-reciprocity)

Observation 11. [Zha19] If $*_{c}$ satisfies ${ }_{{ }_{c}}$-closure, relative success, regularity and reciprocity, then ${ }^{*}$ satisfies:

$$
\text { If } A \equiv B \text {, then } K *_{c} A=K *_{c} B\left(*_{c}-\text { syntax irrelevance }\right)
$$

An axiomatic characterization was obtained for finite languages:

Theorem 38. [Zha19] Let $\mathcal{L}$ be a finite language. Then $*_{c}$ satisfies $\left(*_{c} 1\right)$ through $\left(*_{c} 5\right)$ iff it is a choice revision based on some relational model. 
Additional postulates for choice revision were also studied because the ones listed above do not meet all the plausible properties for the operation:

If $A \neq \varnothing$, then $A \cap\left(K *_{c} A\right) \neq \varnothing\left(*_{c}\right.$-success $)$

If $A$ is consistent, then $K{ }^{*}{ }_{c} A$ is consistent ( ${ }^{*}$-weak consistency)

If $A=\varnothing$, then $K *_{c} A=K\left(*_{c}\right.$-vacuity $)$

The relational model previously defined is not sufficient to satisfy the new properties listed above. The representation theorem below was proposed in order to relate these properties:

Theorem 39. [Zha19] Let $\mathcal{L}$ be a finite language and $*_{c}$ some revision operation on $K \subseteq \mathcal{L}$. Then, ${ }^{*}$ satisfies ${ }^{*}{ }_{c}$-closure, ${ }^{*}$-success, ${ }^{*}{ }_{c}$-vacuity, ${ }^{*}$-confirmation, ${ }_{c}$-reciprocity and ${ }^{*}{ }_{c}$-weak consistency iff it is a choice revision determined by some relational model which satisfies the following two conditions:

$$
\begin{aligned}
& (\mathbb{X} 3) C n(\{\perp\}) \in \mathbb{X} \\
& (\leqq 3) \mathbb{X}^{\mathfrak{B} \varphi} \neq \varnothing \text { and } \mathbb{X}_{<}^{\mathfrak{B} \varphi}<C n(\{\perp\}) \text { for every } \varphi \text { such that } \varphi \text { is consistent. }
\end{aligned}
$$

The new conditions above allow for inconsistent outputs and also guarantees that if there is a possible consistent output then it will be preferred over the inconsistent one.

\subsubsection{Impossibility of Reduction}

Zhang [Zha19] shows that, in general, choice revision by a finite set $A$ cannot be reduced to a selective revision by $\bigwedge A$ :

Example 1. [Zha19] Let $\circ$ be a selective revision operator (Definition 22). It is assumed that $\circ$ satisfies extensionality. Then, $K \circ \bigwedge\{\varphi, \neg \varphi\}$ should be equal to $K \circ \bigwedge\{\psi, \neg \psi\}$. Nevertheless, it is improbable that a choice revision by $\{\varphi, \neg \varphi\}$ should always coincide with that by $\{\psi, \neg \psi\}$ for all $\varphi$ and $\psi$.

Similarly, choice revision cannot be performed by AGM revision by the disjunction of all the sentences of the input (given that AGM revision also satisfies extensionality).

\subsubsection{Making up One's Mind}

There is a variation for multiple revision called called making up one's mind ( $\left.{ }_{\neg}\right)$. It is designed to represent the mechanism of choosing between believing in a formula and in its negation. The usual select-and-intersect belief revision technique is not valid for it but a possible representation is by choice revision: $K \star_{\neg} \alpha=K{ }^{*}{ }_{c}\{\alpha, \neg \alpha\}$. Applying a descriptor revision approach, we have:

Definition 68. [Han17] Let $\circ$ be a descriptor revision on the belief set $K$. The sentential operation * on $K$ such that

$$
K \text { * } \alpha=K \circ\left\{\mathfrak{B}_{\alpha} \vee \mathfrak{B}_{\neg \alpha}\right\} \text { for all } \alpha \in \mathcal{L}
$$

is the operation of resolution (making up one's mind) that is derivable from $\circ$. 
For more information about making up one's mind and the properties of its relation with descriptor revision, the reader is referred to [ZH15].

\subsection{The Multiple Believability Relations Approach}

In the same article [Zha19], a second modelling for choice revision was investigated, this time based on Multiple Believability Relations and without assuming a finite language. First of all, we need the definition of believability relation.

Definition 69. [Han14b] Let $K$ be a belief set, $\circ$ be a descriptor revision on $K, \mathbb{X}$ its outcome set, $\leqq$ (with the strict part $<$ ) a total ordering on $\mathbb{X}$ with $K$ as its minimal element, and $\leq a$ relation of epistemic proximity. Then the relation $\leq$ on sentences, such that $\alpha \leq \beta$ iff $\mathfrak{B}_{\alpha} \leq \mathfrak{B}_{\beta}$, is the believability relation that is based on $\leq$.

Instinctively, $\alpha \leq \beta$ indicates that the agent is at least as inclined to believing $\alpha$ as to believing $\beta$. This relation can be generalized to the multiple case:

Definition 70. [Zha19] A multiple believability relation $\leq_{*}$ is a binary relation on the set of all finite subsets of $\mathcal{L}$ satisfying $\alpha \leq \beta$ iff $\{\alpha\} \leq_{*}\{\beta\}$.

One of the ways of proceeding with the generalization described above (to the multiple case) is defining choice multiple believability relations (denoted by $\leq_{c}$ with symmetric part $\simeq_{c}$ and strict part $<_{c}$ ). $A \leq_{c} B$ indicates that it is easier for an agent to absorb the plausible information in $A$ than that in $B$.

Some postulates for believability relations were defined in [Zha17] and generalized for multi-believability relations in [Zha19]. Let $A, B$ and $C$ be finite sets and $A \otimes B=$ $\{\alpha \wedge \beta \mid \alpha \in A$ and $\beta \in B\}$. Then:

( $\leq_{c}$-transitivity) If $A \leq_{c} B$ and $B \leq_{c} C$, then $A \leq_{c} C$.

( $\leq_{c}$-weak coupling) If $A \simeq_{c} A \oplus B$ and $A \simeq_{c} A \oplus C$, then $A \simeq_{c} A \oplus(B \oplus C)$.

$\left(\leq_{c}\right.$-coupling) If $A \simeq_{c} B$, then $A \simeq_{c} A \oplus B$.

( $\leq_{c}$-counter dominance) If for every $\beta \in B$ there exists $\alpha \in A$ such that $\alpha \in C n(\{\beta\})$, then $A \leq_{c} B$.

( $\leq_{c}$-minimality) $A \leq_{c} B$ for all $B$ iff $A \cap K \neq \varnothing$.

( $\leq_{c}$-maximality) If $B$ is not empty and $A \leq B$ for all non-empty $A$, then $B \equiv\{\perp\}$.

( $\leq_{c}$-completeness) $A \leq B$ or $B \leq A$.

Two other postulates on multi-believability relations were proposed:

$\left(\leq_{c}\right.$-determination) $A \leq \varnothing$ for every non-empty $A$.

( $\leq_{c}$-union) $A \leq_{c} A \cup B$ or $B \leq_{c} A \cup B$.

The postulate of union indicates that to accept part of a non-empty $A$ corresponds to accept some single sentence in $A$. This is conceivable if we presume that the agent is hugely cautious to the new information. 
A construction for this operation was proposed and axiomatically characterized.

\subsubsection{Construction of Choice Revision}

From [Zha17] we have singleton revision * constructed from a believability relation:

$$
\langle\leq \text { to *〉 } K * \alpha=\{\beta \mid \alpha \simeq \alpha \wedge \beta\}
$$

$\alpha \simeq \alpha \wedge \beta$ can be interpreted as that the agent will consequently accept $\beta$ if $\alpha$ is accepted. Analogously, choice revision can be achieved from multi-believability relations:

Definition 71. [Zha19] Let $\leq_{c}$ be some multi-believability relation. A choice revision ${ }^{*}$ on $K$ is based on (or determined by) $\leq_{c}$ iff for any finite $A$ :

$$
\left\langle\leq_{c} \text { to } *_{c}\right\rangle \quad K *_{c} A= \begin{cases}\left\{\alpha \mid A \simeq_{c} A \otimes \alpha\right\} & \text { if } A<_{c} \varnothing \\ K & \text { otherwise }\end{cases}
$$

For this construction, the following representation results were obtained:

Theorem 40. [Zha19] Let $*_{c}$ be some choice revision on $K$. Then $*_{c}$ satisfies $\left(*_{c} 1\right)$ through $\left(*_{c} 5\right)$ iff it is determined by some multi-believability relation $\leq_{c}$ satisfying $\leq_{c}$-transitivity, $\leq_{c}$-weak coupling, $\leq_{c}$-counter-dominance, $\leq_{c}$-minimality and $\leq_{c}$-union.

Theorem 41. [Zha19] Let $*_{c}$ be some choice revision on $K$. Then $*_{c}$ satisfies $*_{c}$-closure, $*_{c}-$ success, $*_{c}$-vacuity, ${ }_{c}$-confirmation, ${ }_{{ }_{c}}$-reciprocity and ${ }_{*_{c}}$-weak consistency iff it is determined by some standard multi-believability relation.

\subsection{The Semi-revision Approach}

The operators defined in [FKIS02], proposed for belief bases, work with partial acceptance in the following way: for a belief base $B$ and an input set $A$, the incoming set is initially accepted and, then, all possible inconsistencies of $B \cup A$ are removed. At the same time, part of the proposal involves to store, in a backup set, the beliefs to be removed from the belief base. They could work as defeasible rules or assumptions.

\subsubsection{Explanations}

Unlike the most common approaches, the input sets considered in this framework are explanations. An explanation contains an explanans (the beliefs that support a consequence) and an explanandum (the final consequence of the explanans). So each explanation is a set of sentences with some restrictions.

Definition 72. [FKIS02] The set $A$ is an explanation for the sentence $\alpha$ iff the following properties are satisfied:

(deduction) $\alpha \in C n(A)$

(consistency) A is consistent

(minimality) If $B \subset A$ then $\alpha \notin C n(B)$ 


\section{(informational content) $C n(A) \nsubseteq C n(\alpha)$}

The relation $A$ explains $\alpha$ will be noted as $A \longmapsto \alpha$.

The motivation behind this structure is the fact that it does not seem rational for an agent to absorb any external belief without evaluating it, which demands an explanation to support the provided belief, especially if the new information is not consistent with its own set of beliefs. If the explanation endures the analysis, the new belief, or its explanation, or both are absorbed by the knowledge base.

\subsubsection{A Revision Operator for Explanations}

In order to explore this kind of behavior, the authors generalized the framework of semi-revision from [Han97] to define an operator ? $^{+}$that supports sets of sentences (explanations) as input and works as a middle form between semi-revision and merge [Fuh97], which is an operator that performs a symmetric revision of two arbitrary sets of sentences. The postulates proposed for the new framework are the following:

(inclusion) $B ?^{+} A \subseteq B \cup A$

(vacuity 1) If $B \cup A$ is inconsistent then $B ?^{+} A=B \cup A$

(vacuity 2) If $A \subseteq B$ and $B$ is consistent then $B ?^{+} A=$

(weak success) If $B \cup A$ is consistent then $A \subseteq B ?^{+} A$

(stability) If $A \subseteq B$ and $B$ is consistent then $A \subseteq B ?^{+} A$

(weak consistency) If $A$ is consistent then $B ?^{+} A$ is consistent

(consistency preservation) If $B$ is consistent then $B ?^{+} A$ is consistent

(consistency) $B ?^{+} A$ is consistent

(core retainment) If $\alpha \in(B \cup A) \backslash\left(B ?^{+} A\right)$ then there is a set $H$ such that $H \subseteq(B \cup A)$, $H$ is consistent but $H \cup\{\alpha\}$ is inconsistent

(relevance) If $\alpha \in(B \cup A) \backslash\left(B ?^{+} A\right)$ then there is a set $H$ such that $B ?^{+} A \subseteq H \subseteq(B \cup A)$, $H$ is consistent but $H \cup\{\alpha\}$ is inconsistent

(congruence) If $B \cup A=B \cup C$ then $B ?^{+} A=B ?^{+} C$

(fairness) If $A$ and $C$ are consistent and for all $H \subseteq B$ it holds that $(H \cup A)$ is inconsistent iff $(H \cup C)$ is inconsistent, then $(B \cup A) \backslash\left(B ?^{+} A\right)=(B \cup C) \backslash\left(B ?^{+} C\right)$

(reversion) If $B \cup A$ and $B \cup C$ have the same minimally inconsistent subsets then $(B \cup A) \backslash\left(B ?^{+} A\right)=(B \cup C) \backslash\left(B ?^{+} C\right)$

(weak monotony) If $A \subseteq C$ and $B \cup C$ is consistent then $B ?^{+} A \subseteq B ?^{+} C$

Two constructions were proposed, one based on kernel sets and the other based on remainder sets, and both of the them were axiomatically characterized. The definitions of kernel and remainder sets, incision and selection functions are the same as in Chapter 2. We show below the definitions of the two constructions and their respective representation theorems: 
Definition 73. [FKIS02] Let $B$ and $A$ be sets of sentences and $\sigma$ an external incision function for $B$. The operator ? $+^{+}$of multiple kernel semi-revision is defined as $B ?^{+} A=(B \cup A) \backslash \sigma((B \cup$ A) $\Perp \perp)$.

Theorem 42. [FKIS02] Let B be a belief base. The operator $?^{+}$is a multiple kernel semirevision iff it satisfies inclusion, consistency, core-retainment and reversion.

Definition 74. [FKISO2] Let $B$ and $A$ be sets of sentences and $\gamma$ a selection function for $B$. The operator ? ${ }^{+}$of multiple partial meet semi-revision is defined as $B ?^{+} A=\bigcap \gamma((B \cup A) \perp \perp)$.

Theorem 43. [FKIS02] Let B be a belief base. The operator ? ${ }^{+}$is a multiple partial meet semi-revision iff it satisfies inclusion, consistency, relevance and reversion.

The postulates below show possible relations between explanans and their respective explanandum. Let $B$ be a belief base, ? ${ }^{+}$a (kernel/partial meet) revision operator by a set of sentences for $B, A$ be explanans, and $\alpha$ be a sentence of the language. Then we have:

(explanans inclusion) If $A \longmapsto \alpha$ and $A \subseteq B ?^{+} A$ then $B ?^{+} A \vdash \alpha$

(weak success 2) If $A \longmapsto \alpha$ and $B \cup A \nvdash \perp$ then $B$ ? $^{+} A \vdash \alpha$

(constrained success) If $A \longmapsto \alpha$ and $B \nvdash \neg \alpha$ then $B ?^{+} A \vdash \alpha$

(expansion) If $A \longmapsto \alpha$ and $B \vdash \alpha$ then $B$ ? $^{+} A \vdash \alpha$

Proposition 4. [FKISO2] If $?^{+}$is a multiple (kernel/partial meet) revision operator, then it satisfies explanans inclusion.

We also observe here that there are different degrees of acceptance of the explanans and the explanandum. The explanans can be explicitly incorporated to the revised set but, on the other hand, the explanandum may be only a conclusion obtained from the explanans without explicitly being absorbed.

Proposition 5. [FKIS02] If $?^{+}$is an operator of multiple semi-revision, then in general it does not satisfy neither constrained success nor expansion.

\subsubsection{Belief Revision in Argumentative Systems}

In order to start, we need the definition of defeasible conditionals. They are conditionals in which, if the first half is true then commonly the second half is true. The sentence $\alpha \succ \beta$ is understood as "if $\alpha$ holds then commonly $\beta$ holds" or "if $\alpha$ is true then usually $\beta$ is true".

In this context, the authors proposed a framework for a revision operator by a set of sentences that produces defeasible conditionals as a product of the revision process. The epistemic state of the agent is described by a tuple of the form $[[K, \Delta]]$ (the knowledge structure) where $K$ is a subset of $\mathcal{L}^{+}$and $\Delta$ is a set defined this way: $\Delta=\left\{\alpha \succ \beta: \alpha, \beta \in \mathcal{L}^{+}\right\}$. $K$ represents the strong or undefeasible knowledge and $\Delta$ is the defeasible knowledge. In addition, the knowledge is represented with a language that is more expressive than the propositional one, with the purpose of differentiating between two kinds of beliefs: particular beliefs $\left(K_{P}\right.$, primarily represented by ground facts such as bird(tweety) or greater $\left.(3,2)\right)$ 
and general beliefs ( $K_{G}$, in order to refer to collections of objects and closed material implications, such as $\forall X(\operatorname{bird}(X) \rightarrow f$ lies $(X)))$.

Their proposal is that a defeasible rule like $\alpha \succ \beta$ in $\Delta$ is the conversion of some rule $\alpha \rightarrow \beta$ formerly present in the strong knowledge but rejected by some change operator. Rather than eliminating it completely, the authors proposed to retain a syntactic transformation of it in a separate set. They defined two types of transformation:

Definition 75. [FKISO2] Let $\delta=\left(\forall X_{1}, \ldots, X_{n}\right)(\alpha \rightarrow \beta)$ be a material implication in $\mathcal{L}^{+} . A$ positive transformation of $\delta$, noted by $T^{+}(\delta)$, is a sentence of the form $\alpha>\beta$; a negative transformation of $\delta$, noted by $T^{-}(\delta)$, is a sentence of the form $\neg \beta \succ \neg \alpha$.

Now, the revision operator is defined:

Definition 76. [FKISO2] Let $[[K, \Delta]]$ be a knowledge structure, $\circ$ be an operator of kernel (partial meet) revision by a set of sentences for $K$ and $A$ be a set of sentences. The kernel (partial meet) composed revision of $[[K, \Delta]]$ with respect to $A$ is defined as $[[K, \Delta]] \star A=\left[\left[K^{\prime}, \Delta^{\prime}\right]\right]$ such that $K^{\prime}=K \circ A$ and $\Delta^{\prime}=\Delta \cup \Delta_{1}^{\prime} \cup \Delta_{2}^{\prime}$ where:

$$
\begin{aligned}
& \Delta_{1}^{\prime}=\left\{\text { true } \succ \alpha: \alpha \in\left(K_{P} \backslash K \circ A\right)\right\} \\
& \Delta_{2}^{\prime}=\left\{T^{+}(\alpha): \alpha \in\left(K_{G} \backslash K \circ A\right)\right\} \cup\left\{T^{-}(\alpha): \alpha \in\left(K_{G} \backslash K \circ A\right)\right\}
\end{aligned}
$$

$K^{\prime}$ is the set of revised undefeasible beliefs. $\Delta_{1}$ and $\Delta_{2}$ involve the transformation of the beliefs removed from $K$ in defeasible rules: the particular ones are transformed and stored in $\Delta_{1}$ and the general ones are transformed and stored in $\Delta_{2}$. Since a particular belief $\alpha$ is not of implication kind, its transformation is defined as true $\succ \alpha$. On the other hand, general beliefs suffer both positive and negative transformations.

This framework has two advantages: the dynamic classification of beliefs (because the beliefs are dynamically classified as undefeasible or defeasible) and minimal change (considering that it maintains as much old information as possible). However, in real implementations there may be memory limitations.

Regarding the issue of deciding when an information is undefeasible or defeasible, the viewpoint of the authors is that the knowledge is undefeasible until we find out new information inconsistent with it.

\subsection{Selective Revision and Deductive Argumentation}

As shown in Section 2.7.2, we have from [FH99] a two-step revision approach called Selective Revision. However, we do not have an explicit implementation of the transformation function. So, the authors in $\left[\mathrm{KTF}^{+} 11\right]$, proposed a concrete implementation of a transformation function using Deductive Argumentation[Dun95, BH01] as the tool to evaluate the desirability of new information for a belief base.

Briefly, a deductive argumentation theory is a set of propositional sentences and an argument for some sentence $\phi$ is a minimal proof for $\phi$. 
The transformation function proposed in $\left[\mathrm{KTF}^{+} 11\right]$ determines, for each single piece of information, if it will be admitted for revision or not and, if so, it will be completely absorbed or will be weakened. The decision is based on its argumentative evaluation, i.e., whether $\alpha \in \Phi$ is justifiable with respect to $\Phi$. That is why the authors considered a multiple revision scenario - the new information is allowed to contain arguments.

For non-prioritized multiple base revision, the authors recover four postulates from [FKIS02]: inclusion, vacuity 1, weak consistency and relevance. They also considered weakened versions of success and extensionality. Let $B$ be a belief base, $A$ be a set of sentences, * $p$ be a multiple package base revision operator and $\odot$ be a (generic) non-prioritized multiple belief revision operator:

Weak success: If $B \cup A$ is consistent then $A \subseteq C n(B \odot A)$

Consistent expansion: If $B \nsubseteq B \odot A$ then $B \cup(B \odot A)$ is inconsistent

Weak extensionality: $:^{2}$ If $A \cong \cong^{p} C$ then $B{ }_{p} A \equiv^{p} B *_{p} C$

Then, the properties that a (generic) non-prioritized multiple revision operator is expected to satisfy are listed below in a generic way:

Definition 77. $\left[\mathrm{KTF}^{+} 11\right]$ A revision operator $\odot$ is called non-prioritized multiple base revision operator if $\odot$ satisfies inclusion, weak consistency, weak extensionality, weak success and consistent expansion.

Adapting Selective Revision to the multiple case, a new operator was defined:

Definition 78. [KTF 11$]$ Let $B$ be a belief base, $A$ be a set of sentences, ${ }^{*} p$ be some multiple package base revision, and $f$ be a transformation function. Then $\odot$ is a Multiple Selective Base Revision defined as: $B \odot A=B{ }_{p} f(A)$.

Some of the properties for the transformation function defined in [FH99] were rephrased in order to work in this new context:

Inclusion: $f(A) \subseteq A$

Weak inclusion: If $B \cup A$ is consistent then $f(A) \subseteq A$

Extensionality: If $A \equiv^{p} A^{\prime}$ then $f(A) \equiv^{p} f\left(A^{\prime}\right)$

Consistency preservation: If $A$ is consistent then $f(A)$ is consistent

Consistency: $f(A)$ is consistent

Maximality: $f(A)=A$

Weak Maximality: If $B \cup A$ is consistent then $f(A)=A$

In addition, a new property was proposed:

Weak Extensionality: If $A \cong^{P} A^{\prime}$ then $f(A) \cong^{P} f\left(A^{\prime}\right)$

\footnotetext{
${ }^{2}$ Two sets of sentences $A, C$ are equivalent $(A \equiv P C)$ iff it holds that $A \subseteq C n(C)$ and $C \subseteq C n(A)$. The sets have an equivalence relation $\left(A \cong \cong^{p} C\right)$ iff there is a bijection $\sigma: A \rightarrow C$ such that for every $\alpha \in A$ it holds that $\alpha \equiv p \quad \sigma(\alpha)$.
} 
Proposition 6. [KTF $\left.{ }^{+} 11\right]$ Let ${ }_{p}$ be a multiple package base revision operator and let $f$ satisfy inclusion, weak maximality, consistency preservation and weak maximality. Then $\odot$ defined via Definition 78 is a non-prioritized multiple base revision operator.

The proposition above states that the Multiple Selective Base Revision operator proposed in Definition 78 satisfies inclusion, weak consistency, weak extensionality, weak success and consistent expansion.

Based on the deductive argumentation framework from [BH01], the authors implemented two different transformation functions and showed that the Multiple Selective Base Revision operators defined from them are non-prioritized multiple base revision operators.

\subsection{Dealing with Inconsistent Inputs}

As shown in Section 3.4, in [FKIRS12] when the input set is inconsistent the agent does nothing, preserving all of its previous beliefs. Other works on prioritized revision consider that if the input set is inconsistent then the result of revision will be inconsistent. In [Del11], however, Delgrande observes that, even if the input is inconsistent, it may have consistent individual pieces of information and, hence, in an alternative way, maximal consistent subsets of the input may lead to possible sentences for the revision process. The intuition behind this idea is that, although it is not rational to accept the whole set if it is inconsistent, it is reasonable that an agent would want to absorb a maximal amount of pieces of information (an application to the input formulas of an analogue of the principle of informational economy). The plausibility of such possible sentences (from the maximal consistent subsets) is given by a faithful ranking associated to the agent, which attributes a believability level for every formula. This concept of believability is different from the agent's plausibility ordering on formulas.

In his approach, instead of belief sets, the author considers belief states as objects of revision. The belief set corresponding to a belief state $\mathcal{K}$ is given by $\operatorname{Bel}(\mathcal{K})$. Katsuno and Mendelzon [KM91b] have demonstrated that a necessary and sufficient condition for designing an AGM revision operator is that a belief state $\mathcal{K}$ can lead to, as its preferential information, a total preorder on the set of possible worlds ${ }^{3}$ :

Definition 79. [KM91b] A faithful assignment is a function that maps each belief state $\mathcal{K}$ to a total preorder $\leq \mathcal{K}$ on $\mathcal{M}$ such that for any possible worlds $\mathcal{V}_{1}, \mathcal{V}_{2}$ :

- If $\mathcal{V}_{1}, \mathcal{V}_{2} \vDash \operatorname{Bel}(\mathcal{K})$ then $\mathcal{V}_{1}={ }_{\mathcal{K}} \mathcal{V}_{2}$

- If $\mathcal{V}_{1} \vDash \operatorname{Bel}(\mathcal{K})$ and $\mathcal{V}_{2} \# \operatorname{Bel}(\mathcal{K})$, then $\mathcal{V}_{1}<_{\mathcal{K}} \mathcal{V}_{2}$

The derived total preorder is identified as the faithful ranking corresponding to, or induced by $\mathcal{K}$.

\footnotetext{
${ }^{3}$ The definition of possible world is given in Chapter 2, Section 2.6.6.
} 


\subsubsection{The Approach}

First, it is necessary to define maximum consistent subsets of a set of formulas. The definition is the same as remainder in [AM81] (Definition 1) but considering $\alpha=\perp$. Then, the set of the maximum consistent subsets of a set $A$ of formulas is given by $\operatorname{Con}(A)=A \perp \perp$.

\section{Proposition 7. [Del11]}

- $\operatorname{Con}(A)=A$ iff $A H \perp$

- $\operatorname{Con}(A)=\varnothing$ iff $\forall \alpha \in A, \alpha \vdash \perp$ (i.e., $A$ is strongly inconsistent)

Regarding the properties of this new variant of the revision operation, Delgrande extended the set revision postulates from [Lin91, Pep04] in order to allow for inconsistent inputs (and also adapting for belief states), referring to them as the extended set revision postulates. Let $A$ be a finite set:

$(\mathcal{K} \otimes 1) \operatorname{Cn}(\operatorname{Bel}(\mathcal{K} \otimes A))=\operatorname{Bel}(\mathcal{K} \otimes A)$

$(\mathcal{K} \otimes 2) \bigvee \operatorname{Con}(A) \in \operatorname{Bel}(\mathcal{K} \otimes A)$

$(\mathcal{K} \otimes 3) \operatorname{Bel}(K \otimes A) \subseteq \bigcap_{A^{\prime} \in \operatorname{Con}(A)}\left(\operatorname{Bel}\left(\operatorname{Cn}\left(\mathcal{K} \cup A^{\prime}\right)\right)\right)$

$(\mathcal{K} \otimes 4)$ If $\operatorname{Cn}(\operatorname{Bel}(\mathcal{K}) \cup \bigvee \operatorname{Con}(A))$ is consistent, then $\bigcap_{A^{\prime} \in \operatorname{Con}(A)} \operatorname{Cn}\left(\operatorname{Bel}(\mathcal{K}) \cup A^{\prime}\right) \subseteq$ $\operatorname{Bel}(\mathcal{K} \otimes A)$

$(\mathcal{K} \otimes 5) \operatorname{Bel}(\mathcal{K} \otimes A)$ is inconsistent only if $A$ is strongly inconsistent

$(\mathcal{K} \otimes 6)$ If $A_{1} \forall \perp$ and $A_{1} \equiv A_{2}$, then $\operatorname{Bel}\left(\mathcal{K} \otimes A_{1}\right)=\operatorname{Bel}\left(\mathcal{K} \otimes A_{2}\right)$

$\left(\mathcal{K} \otimes 6^{\prime}\right)$ If $A_{1}$ and $A_{2}$ are strongly equivalent, then $\operatorname{Bel}\left(\mathcal{K} \otimes A_{1}\right)=\operatorname{Bel}\left(\mathcal{K} \otimes A_{2}\right)$

$(\mathcal{K} \otimes 7)$ If $A_{1} \forall \perp$ then $\operatorname{Bel}\left(\mathcal{K} \otimes\left(A_{1} \cup A_{2}\right)\right) \subseteq \operatorname{Cn}\left(\operatorname{Bel}\left(\mathcal{K} \otimes A_{1}\right) \cup A_{2}\right)$

$(\mathcal{K} \otimes 8)$ If $A_{1} \forall \perp$ and $\operatorname{Bel}\left(\mathcal{K} \otimes A_{1}\right) \cup A_{2}$ is consistent, then $\operatorname{Cn}\left(\operatorname{Bel}\left(\mathcal{K} \otimes A_{1}\right) \cup A_{2}\right) \subseteq$ $\operatorname{Bel}\left(\mathcal{K} \otimes\left(A_{1} \cup A_{2}\right)\right)$

The proposition below follows from $(\mathcal{K} \otimes 3)$ and $(\mathcal{K} \otimes 4)$ :

Proposition 8. [Del11] Let $\mathrm{A}=\left\{A^{\prime} \in \operatorname{Con}(A) \mid \operatorname{Cn}\left(\operatorname{Bel}(\mathcal{K}) \cup A^{\prime}\right) \forall \perp\right\}$. If $\mathbb{A} \neq \varnothing$ then $\operatorname{Bel}(\mathcal{K} \otimes A)=\bigcap_{A^{\prime} \in \mathbb{A}} \operatorname{Cn}\left(\operatorname{Bel}(\mathcal{K}) \cup A^{\prime}\right)$.

Delgrande also extended the set revision postulates by the following one:

$(\mathcal{K} \otimes P P)$ Let $A_{1} \subseteq A$ where $A_{1} \cup \neg\left(A \backslash A_{1}\right) H \perp$. Then $\operatorname{Bel}\left(\mathcal{K} \otimes A \otimes \neg\left(A \backslash A_{1}\right)\right)=$ $\operatorname{Bel}\left(\mathcal{K} \otimes\left(A_{1} \cup \neg\left(A \backslash A_{1}\right)\right)\right) .{ }^{4}$

$(\mathcal{K} \otimes P P)$ represents the requirement that after revising by a set of formulas, and in the sequence by the negations of a subset of those formulas, the remaining formulas, when possible, will continue to be regarded as true. The condition below is on a faithful ranking, analogous to $(\mathcal{K} \otimes P P)$ :

\footnotetext{
${ }^{4}$ Here, for a set $A$, the definition of $\neg A$ is the same as in [Fuh88], i.e., $\neg A=\{\neg \alpha: \alpha \in A\}$.
} 
$(P P)$ Let $A_{1} \subseteq A$ where $A_{1} \cup \neg\left(A \backslash A_{1}\right) \forall \perp$. Then $\min \left(\operatorname{Mod}\left(A_{1} \cup \neg\left(A \backslash A_{1}\right)\right), \leq \mathcal{K}\right)=$ $\min \left(\operatorname{Mod}\left(\neg\left(A \backslash A_{1}\right)\right), \leq \mathcal{K}_{\otimes \mathcal{A}}\right)$.

A factoring result was also obtained for maximum consistent sets:

Proposition 9. [Del11]

$\left(\mathcal{K} \otimes F^{n}\right)$ Let $\operatorname{Con}(A)=\left\{A_{1}, \ldots, A_{n}\right\} \neq \varnothing$. Then for some $\mathrm{A}$ where $\mathrm{A} \subseteq \operatorname{Con}(A), \operatorname{Bel}(\mathcal{K} *$ $A)=\bigcap_{A^{\prime} \in \mathbb{A}} \operatorname{Bel}\left(\mathcal{K} * A^{\prime}\right)$

Extending a result from [KM91b], Delgrande obtained the following representation result:

Theorem 44. [Del11] A revision operator $\otimes$ satisfies the extended set revision postulates iff there exists a faithful assignment that maps $\mathcal{K}$ to the faithful ranking $\leq \mathcal{K}$ such that for any finite set of formulas $A$ :

$$
\operatorname{Bel}(\mathcal{K} \otimes A)=\mathcal{T}(\min (\operatorname{Mod}(\bigvee \operatorname{Con}(A)), \leq \mathcal{K}))
$$

Combining this approach with the one presented in [DJ08] in order to get recommended "preferred" basic approach to revising by a set of formulas:

Theorem 45. [Del11] A revision operator $\otimes$ satisfies the extended set revision postulates together with $(\mathcal{K} \otimes P P)$ iff there is a faithful assignment whose corresponding faithful ranking $\leq \mathcal{K}$ satisfies $(P P)$, and where for any finite set of sentences $A$ :

$$
\operatorname{Bel}(\mathcal{K} \otimes A)=\mathcal{T}(\min (\operatorname{Mod}(\bigvee \operatorname{Con}(A)), \leq \mathcal{K}))
$$

So, in this approach, when revising by an input set $A$, an agent should take into account the maximal consistent subsets of $A$ as possible sets for revision, and then choose the most plausible worlds out of these various subsets to represent the revision. The result below was also obtained:

Proposition 10. [Del11] If $A^{\prime} \in \operatorname{Con}(A)$ then $\operatorname{Bel}\left(\mathcal{K} \otimes A \otimes A^{\prime}\right)=\operatorname{Bel}\left(\mathcal{K} \otimes A^{\prime}\right)$.

\subsubsection{Comparing with Iterated Revision}

The author argues that, if the agent is in a static domain and receives a sequence of input formulas for revision about this domain, there is no argument that the latest one must be accepted. Hence, if the order is insignificant, the input for revision should be the set of such formulas. It promotes a very different perspective of iterated revision: a revision sequence $\mathcal{K} * \alpha_{1} * \ldots * \alpha_{n}$ is more properly considered as the uniterated revision $\mathcal{K} \otimes\left\{\alpha_{1}, \ldots, \alpha_{n}\right\}$. Thus, in theory there is no necessity for iterated revision as assumed by the belief change community, remaining just a pragmatic role for it.

As a final point, if the input set is inconsistent, the approach that reduces multiple revision to a singleton revision by the conjunction of the input sentences would lead to an inconsistency. 


\subsection{The Core Beliefs Approach}

In the literature, we find two approaches for multiple revision which were based on the concept of core beliefs. We can consider it as defined by David Makinson[Mak97]. He proposes a context where the original belief set to be revised has a subset taken as core, which means that it cannot be revised. It is considered immune to revision (or, in his own words, sacrosanct) and it is entirely preserved independently of the new information.

Both of the approaches are characterized axiomatically and also receive two constructions: one operator based on kernel sets and another one on remainder sets. In addition, the approaches use the concept of belief state defined as follows:

Definition 80. [YFW14] $A$ belief state is a pair $S=(B, A)$ satisfying:

1. $A \subseteq B \subseteq \mathcal{L}$

2. A is consistent

3. $A$ is logically closed within $B$, i.e., $C n(A) \cap B \subseteq A$

The set of all belief states is denoted by $\mathcal{B}$. For every $(B, A) \in \mathcal{B}, B$ is called the belief base and A the set of core beliefs.

As common properties, both of the operators satisfy three principles:

- minimal change: the agent should preserve old beliefs as much as it can;

- consistency: the resulting belief state should be consistent after the whole revision process;

- protection: core beliefs should always be preserved.

In the next subsections we are going to bring a quick view of them.

\subsubsection{Evaluative Multiple Revision}

Evaluative Multiple Revision (EMR) is an operation through which the new information, instead of being directly handled, is pre-processed in an evaluation process that takes into account the core beliefs of the agent and, then, the revision is performed. Therefore, it is considered a sort of non-prioritized multiple revision, as the whole new information is not necessarily incorporated. This characterization inserts EMR in the decision + revision variety of non-prioritized belief revision[Han99a], i.e., a two-phase revision process.

The new information is represented by a set of sentences. When it comes to the agent, it is first submitted to a decision module which, using the core beliefs as criteria, performs an evaluation and produces two disjoint sets: one for plausible information and another one for implausible. More formally:

Definition 81. [YJW14] Given a belief state $(B, A)$, an A-evaluation is a pair of sets of formulas in $\mathcal{L}$, denoted by $I \mid P$, satisfying:

1. $I \cup P \neq \varnothing$

2. $A \cup P H \perp$ 


\section{3. $C n(A \cup P) \cap I=\varnothing$}

The set of all $A$-evaluations is denoted by $\mathcal{A}$. As can be inferred from the above definition, $I$ is the set of implausible new information (the Out-set of $I \mid P$ ) while $P$ is the set of plausible new information (the In-set of $I \mid P$ ). The last two conditions can be expressed together by a single one: $C n(A \cup P) \cap(I \cup\{\perp\})=\varnothing$.

Differently from other frameworks, the revision module does not receive a single set of sentences to perform the revision operation. It receives the pair of sets produced by the previous module. The idea is to revise the agent's beliefs by the plausible set and, at the same time, contract them by the implausible set.

So, the EMR operator $\triangleright$ maps a belief state $(B, A)$ and an $A$-evaluation $I \mid P$ to a new belief state, that is, the result of $(B, A) \triangleright I \mid P$ is a pair as well. The first and the second components of the result are represented by $B \triangleright_{A}(I \mid P)$ and $A \triangleleft_{B}(I \mid P)$, respectively.

Some postulates for $\triangleright$ were defined. For all $I\left|P, I_{1}\right| P_{1}, I_{2} \mid P_{2} \in \mathcal{A}$ :

$($ EV-BelState $)(B, A) \triangleright(I \mid P) \in \mathcal{B}$

(EV-Consistency) $B \triangleright_{A}(I \mid P) \forall \perp$

(EV-CoreInvariance) $A \triangleleft_{B}(I \mid P)=C n(A) \cap(B \cup P)$

(EV-Inclusion) $B \triangleright_{A}(I \mid P) \subseteq B \cup P$

(EV-OutSuccess) $C n\left(B \triangleright_{A}(I \mid P)\right) \cap I=\varnothing$

(EV-InSuccess) $P \subseteq B \triangleright_{A}(I \mid P)$

(EV-Protection) $A \subseteq B \triangleright_{A}(I \mid P)$

(EV-Closure) $C n\left(B \triangleright_{A}(I \mid P)\right) \cap B \subseteq B \triangleright_{A}(I \mid P)$

(EV-Vacuity) $C n(B \cup P) \cap(I \cup\{\perp\})=\varnothing \longrightarrow B \cup P \subseteq B \triangleright_{A}(I \mid P)$

(EV-Uniformity) If $\forall X \subseteq B$,

$$
C n\left(X \cup A \cup P_{1}\right) \cap\left(I_{1} \cup\{\perp\}\right) \neq \varnothing \text { iff } C n\left(X \cup A \cup P_{2}\right) \cap\left(I_{2} \cup\{\perp\}\right) \neq \varnothing,
$$

then $B \backslash\left(B \triangleright_{A}\left(I_{1} \mid P_{1}\right)\right)=B \backslash\left(B \triangleright_{A}\left(I_{2} \mid P_{2}\right)\right)$.

(EV-Retainment) If $\varphi \in B \backslash B \triangleright_{A}(I \mid P)$, then there exists $X \subseteq B$ such that

$$
\begin{gathered}
C n(X \cup A \cup P) \cap(I \cup\{\perp\})=\varnothing \\
C n(X \cup\{\varphi\} \cup A \cup P) \cap(I \cup\{\perp\}) \neq \varnothing .
\end{gathered}
$$

(EV-Relevance) If $\varphi \in B \backslash B \triangleright_{A}(I \mid P)$, then there exists $X \subseteq B$ such that $B \triangleright_{A}(I \mid P) \cap B \subseteq$ $X$ and

$$
\begin{gathered}
C n(X \cup A \cup P) \cap(I \cup\{\perp\})=\varnothing \\
C n(X \cup\{\varphi\} \cup A \cup P) \cap(I \cup\{\perp\}) \neq \varnothing .
\end{gathered}
$$




\subsubsection{Evaluative Kernel Sets}

The authors proposed a construction for EMR based on the kernel set constructions, calling it Kernel Evaluative Multiple Revision (KEMR), which is a mixture of multiple contraction (by the Out-set) with prioritized multiple revision (by the In-set).

Definition 82. [Y\}W14] Let $(B, A) \in \mathcal{B}, I \mid P \in \mathcal{A}$. The $I \mid P$-evaluative kernel set for $(B, A)$ denoted by $B \mathbf{\Lambda}_{\perp}^{A}(I \mid P)$, consists of all $X \subseteq B$ satisfying the following conditions:

1. $C n(X \cup A \cup P) \cap(I \cup\{\perp\}) \neq \varnothing$

2. If $X^{\prime} \subset X$ then $C n\left(X^{\prime} \cup A \cup P\right) \cap(I \cup\{\perp\})=\varnothing$

The incision function is similar to the traditional one, except for a explicit condition that avoids excluding the plausible information with the implausible information:

Definition 83. [YFW14] Let $(B, A) \in \mathcal{B}$. A map $\rho: \mathcal{P}(\mathcal{P}(\mathcal{L}) \rightarrow \mathcal{P}(\mathcal{L})$ is an evaluative incision function for $(B, A)$ if for every evaluative kernel set $B \mathbf{\Lambda}_{\perp}^{A}(I \mid P)$ :

1. $\rho\left(B \mathbf{\Delta}_{\perp}^{A}(I \mid P)\right) \subseteq \bigcup\left(\left(B \mathbf{\Delta}_{\perp}^{A}(I \mid P)\right)\right)$

2. If $X \in B \mathbf{\Lambda}_{\perp}^{A}(I \mid P)$ then $\rho\left(\left(B \mathbf{\Delta}_{\perp}^{A}(I \mid P)\right)\right) \cap X \neq \varnothing$

3. If $\psi \in \rho\left(B \mathbf{\Delta}_{\perp}^{A}(I \mid P)\right)$ then $B \backslash \rho\left(B \mathbf{\Delta}_{\perp}^{A}(I \mid P)\right) \cup P \forall \psi$

Then, the construction can be defined as follows:

Definition 84. [YFW14] Let $(B, A) \in \mathcal{B}$ and $\rho$ be an evaluative incision function for $(B, A)$.

1. The KEMR operator generated by $\rho$, denoted by $\triangleright^{\rho}$, is defined by

$$
(B, A) \triangleright^{\rho}(I \mid P)=\left(B^{\prime}, A^{\prime}\right), \forall(I \mid P) \in \mathcal{A},
$$

where $B^{\prime}=B \backslash \rho\left(B \mathbf{\Delta}_{\perp}^{A}(I \mid P)\right) \cup P$ and $A^{\prime}=C n(A) \cap B^{\prime}$.

2. A map $\triangleright$ is called a KEMR operator if there exists an evaluative incision function $\rho$ such that $\triangleright=\triangleright^{\rho}$.

A representation result is shown below:

Theorem 46. [Y\}W14] An operator $\triangleright$ is a KEMR operator iff it satisfies EV-BelState, EVConsistency, EV-CoreInvariance, EV-Inclusion, EV-InSucess, EV-OutSucess, EV-Closure, EVUniformity and EV-Retainment.

\subsubsection{Evaluative Remainder Sets}

Analogously, they proposed a construction for EMR based on the remainder sets, calling it Partial Meet Evaluative Multiple Revision (PMEMR), which again is a mixture of contraction with revision.

Definition 85. [Y\}W14] Let $(B, A) \in \mathcal{B}, I \mid P \in \mathcal{A}$. The $I \mid P$-evaluative remainder set for $(B, A)$ denoted by $B_{\top}^{A}(I \mid P)$, consists of all $X \subseteq B$ satisfying the following conditions:

1. $C n(X \cup A \cup P) \cap(I \cup\{\perp\})=\varnothing$

2. If $X \subset X^{\prime} \subseteq B$ then $C n\left(X^{\prime} \cup A \cup P\right) \cap(I \cup\{\perp\}) \neq \varnothing$ 
The (evaluative) selection function works in the same way as for the traditional construction. Based on these two definitions, the construction can be defined as follows:

Definition 86. [YFW14] Let $(B, A) \in \mathcal{B}$ and $\gamma$ be an evaluative selection function for $(B, A)$.

1. The PMEMR operator generated by $\gamma$, denoted by $\triangleright^{\gamma}$, is defined by

$$
(B, A) \triangleright^{\gamma}(I \mid P)=\left(B^{\prime}, A^{\prime}\right), \forall(I \mid P) \in \mathcal{A},
$$

where $B^{\prime}=\bigcap \gamma\left(B_{T}^{A}(I \mid P)\right) \cup P$ and $A^{\prime}=C n(A) \cap B^{\prime}$.

2. A map $\triangleright$ is called a PMEMR operator if there exists an evaluative selection function $\gamma$ such that $\square=\nabla^{\gamma}$.

A representation result is shown below:

Theorem 47. [Y\}W14] An operator $\triangleright$ is a PMEMR operator iff it satisfies EV-BelState, EVConsistency, EV-CoreInvariance, EV-Inclusion, EV-InSucess, EV-OutSucess, EV-Uniformity and EV-Relevance.

Corollary 1. [Y\}W14] If $\triangleright$ is a PMEMR operator, then it is also a KEMR operator.

\subsubsection{Comparing with Other Approaches}

In the end of the article, EMR is compared with the operations of prioritized multiple revision defined in [FKIRS12] (shown in Section 3.4). Roughly speaking, the operations $*_{\sigma}$ and ${ }_{\gamma}$ are special cases of $\nabla^{\sigma}$ and $D^{\gamma}$, respectively, when the Out-set is empty:

Definition 87. [Y\}W14] Let $(B, A) \in \mathcal{B}, A=C n(\varnothing) \cap B$ and $\triangleright$ be an EMR operator for $(B, A)$. The revision operator generated by $\triangleright$, denoted by ${ }^{*} \triangleright$, is defined as follows. For all $P \in \mathcal{P}(\mathcal{L}) \backslash\{\varnothing\}:$

$$
B *_{\triangleright} P= \begin{cases}B \triangleright_{A}(\varnothing \mid P) & \text { if } P \forall \perp \\ B & \text { otherwise }\end{cases}
$$

Theorem 48. [Y\}W14] Let $(B, A) \in \mathcal{B}, A=C n(\varnothing) \cap B$ and $\triangleright$ be an EMR operator for $(B, A)$.

1. If $\triangleright$ is a KEMR operator, then ${ }^{\circ}$ is a prioritized multiple kernel revision operator.

2. If $\triangleright$ is a PMEMR operator, then ${ }^{\star} \triangleright$ is a prioritized multiple partial meet revision operator.

It is also possible to compare EMR with Selective Revision[FH99], an operation for singleton revision. The idea is very similar, given that in both of the approaches the input is treated by a separate mechanism before effectively being used to perform revision. Nevertheless, while the transformation function from Selective Revision may return logical consequences of the input, the decision module from EMR produces subsets of the incoming set. In addition, Selective Revision does not work with core beliefs. Considering these aspects, EMR cannot be considered a generalization of Selective Revision. 


\subsubsection{Rational Metabolic Revision}

Differently from the previous scenario, there are some contexts where the agent cannot identify, initially, the implausible part of the incoming new information. Then, it incorporates all the new beliefs to its corpus of knowledge (expansion), which may cause some belief conflicts that will be useful to detect the implausible information (based on its criteria) and consolidate the belief state. In [Yua17], then, we have the proposal of a new multiple revision operator that works this way and is named metabolic revision. It is inserted, therefore, in the expansion + consolidation variety of non-prioritized belief revision[Han99a].

The name metabolic revision is due to a correlation with body metabolism. If an animal takes some food and, with its bounded rationality, consider it as good to eat, the animal will ingest it and later its body will eliminate some harmful substance or trash by the digestive system. The idea for the operator is to work in a similar manner with new information.

The metabolic revision operator is represented by $\diamond$ and maps a belief state $(B, A)$ and a set of beliefs $D$ to a new belief state $\left(B^{\prime}, A^{\prime}\right)$, i.e., as well as in the previous approach, the outcome of $(B, A) \diamond D$ is also a pair. In the new belief state, the belief base and the set of core belief are represented by $B \diamond_{A} D$ and $A \diamond_{B}$, respectively.

Some postulates for $\diamond$ were defined. For all sets $D, D_{1}, D_{2} \subseteq \mathcal{L}$ :

$($ M-BelState $)(B, A) \diamond D \in \mathcal{B}$

(M-Consistency) $B \diamond_{A}(D) H \perp$

(M-CoreInvariance) $A \diamond_{B} D=C n(A) \cap(B \cup D)$

(M-Inclusion) $B \diamond_{A} D \subseteq B \cup D$

(M-Protection) $A \subseteq B \diamond_{A} D$

(M-RelClosure) $C n\left(B \diamond_{A} D\right) \cap(B \cup D) \subseteq B \diamond_{A} D$

(M-Vacuity) If $B \cup D \forall \perp$, then $B \cup D \subseteq B \diamond_{A} D$

(M-Uniformity) If $B \cup D_{1}$ and $B \cup D_{2}$ have the same minimal subsets inconsistent with $A$, then $\left(B \cup D_{1}\right) \backslash\left(B \diamond_{A} D_{1}\right)=\left(B \cup D_{2}\right) \backslash\left(B \diamond_{A} D_{2}\right)$.

(M-ExIrrelevance) If $A \cup\{\varphi\} \vdash \perp$ for all $\varphi \in\left(\left(B \cup D_{1}\right) \cup\left(B \cup D_{2}\right)\right) \backslash\left(\left(B \cup D_{1}\right) \cap\left(B \cup D_{2}\right)\right)$, then $B \diamond_{A} D_{1}=B \diamond_{A} D_{2}$.

(M-ExRetainment) If $\varphi \in(B \cup D) \backslash B \diamond_{A} D$, then there exists $X \subseteq B \cup D$ such that $X \cup A \forall \perp$ and $X \cup\{\varphi\} \cup A \vdash \perp$.

(M-ExRelevance) If $\varphi \in(B \cup D) \backslash B \diamond_{A} D$, then there exists $X \subseteq B \cup D$ such that $B \diamond_{A} D \subseteq X, X \cup A \forall \perp$ and $X \cup\{\varphi\} \cup A \vdash \perp$.

\subsubsection{Kernel Metabolic Revision}

For this construction, the author applied the definition of $A$-inconsistent kernel set from [FKIRS12], as stated in Definition 48, in order to compute $(B \cup D) \downarrow \downarrow A$. The metabolic 
incision function works in the same way as defined in [YJW14] (Definition 83). So, it is possible to construct the Kernel Metabolic Revision (KMR):

Definition 88. [Yua17] Let $(B, A) \in \mathcal{B}$ and $\theta$ be a metabolic incision function for $(B, A)$.

1. The KMR operator for $(B, A)$ generated by $\theta$, denoted by $\diamond^{\theta}$, is defined by: for all $D \subseteq \mathcal{L}$, $(B, A) \diamond^{\theta} D=\left(B^{\prime}, A^{\prime}\right)$, in which $B^{\prime}=(B \cup D) \backslash \theta((B \cup D) \downarrow \downarrow A)$ and $A^{\prime}=C n(A) \cap B^{\prime}$.

2. An operator $\diamond$ is a KMR operator for $(B, A)$ if there exists a metabolic incision function $\theta$ such that: for all $D \subseteq \mathcal{L},(B, A) \diamond D=(B, A) \diamond^{\theta} D$.

A representation result is shown below:

Theorem 49. [Yua17] Let $(B, A) \in \mathcal{B}$. An operator $\diamond$ is a KMR operator for $(B, A)$ iff it satisfies: M-CoreInvariance, $M$-Inclusion, $M$-Consistency, M-RelClosure, $M$-Uniformity and M-ExRetainment.

\subsubsection{Partial Meet Metabolic Revision}

For this construction, the author applied the definition of $A$-consistent remainder set used in [FKIRS12] (Definition 33), in order to compute $(B \cup D) \downarrow A$. The metabolic selection function works in the same way as the traditional construction. So, it is possible to construct the Partial Meet Metabolic Revision (PMMR):

Definition 89. [Yua17] Let $(B, A) \in \mathcal{B}$ and $\mathrm{Y}$ be a metabolic selection function for $(B, A)$.

1. The PMMR operator for $(B, A)$ generated by $\mathrm{Y}$, denoted by $\diamond^{\mathrm{Y}}$, is defined by: for all $D \subseteq \mathcal{L},(B, A) \diamond^{\mathrm{Y}} D=\left(B^{\prime}, A^{\prime}\right)$, in which $B^{\prime}=\bigcap \mathrm{Y}((B \cup D) \downarrow A), A^{\prime}=C n(A) \cap B^{\prime}$.

2. An operator $\diamond$ is a PMMR operator for $(B, A)$ if there exists a metabolic selection function $\mathrm{Y}$ for $(B, A)$ such that: for all $D \subseteq \mathcal{L},(B, A) \diamond D=(B, A) \diamond^{\mathrm{Y}} D$.

A representation result is shown below:

Theorem 50. [Yua17] Let $(B, A) \in \mathcal{B}$. An operator $\diamond$ is a PMMR operator for $(B, A)$ iff it satisfies: $M$-CoreInvariance, $M$-Inclusion, $M$-Consistency, M-ExIrrelevance and M-ExRelevance.

\subsubsection{Comparing with Semi-revision}

As observed by Yuan, semi-revision [Han97] is, apparently, a particular case of metabolic revision when $A$ is empty and $D$ is a singleton. Nevertheless, it is not the case. While it is possible to establish an interrelation between two semi-revisions of different belief bases, metabolic revision is for a fixed belief state, i.e., properties for the interrelation between two metabolic revisions on different belief states were not defined.

In [Fuh97], Merging appears as a generalization of the Consolidation operation through the combination of two belief bases into one. In [KP02b], it was extended to Merging with integrity constraints (IC merging) and in [FKIRS12] we have two constructive approaches to merging based on kernel and remainder sets. The combination of different belief bases through a change process that is affected by some constraints is a great similarity between MR and IC merging. However, in [Yua17] Yuan points out some differences, among which we refer the symmetry aspect (merging is a symmetrical change, without the duality 
between belief states and a new piece of information, while MR is not), their formulations (IC merging involves more than two inputs and MR has only two inputs) and the constraints required (IC merging demands $\mu$ to be a logical consequence of the final result whereas MR demands the core beliefs to be a subset of the new belief base).

\subsection{Final Remarks}

Most of the approaches presented in this chapter involve only two of the epistemic models referred in the Introduction: belief sets and belief bases. While in [Fuh88, Del11, Han17, Zha19] the focus was on belief sets, in [FKIS02, $\mathrm{KTF}^{+} 11$, YJW14, Yua17, Zha18] they worked with belief bases. Only in [Del11] the author uses possible worlds due to the definition of faithful assignment.

In relation to the underlying logic involved, all of them used classical propositional logic. With respect to the finiteness of the input, only the approaches in [YJW14, Yua17] considered the possibility for infinite sets.

Regarding constructions, in [Fuh88, Zha18] we have partial meet, while in [FKIS02, YJW14, Yua17] we have partial meet and kernel and. The authors in [RGW $\left.{ }^{+} 20\right]$ worked with deductive argumentation, the ones in [Han17, Zha19] with descriptor revision and Delgrande [Del11] with remainder set and faithful assignments and. Finally, Zhang [Zha19] also works with multiple believability relations. Regarding postulates, the similarity between the set of properties used in two distinct works is greater if they are based on the same epistemic model. On the other hand, some postulates are specific for the involved approach, such as the ones for selective revision, choice through descriptor revision or core beliefs. In this regard, the postulates proposed for non-prioritized multiple revision operations are more diverse than in the preceding chapter.

As well as in the previous chapter, Table 4.1 summarizes the main characteristics of the different approaches analyzed in this chapter regarding the epistemic model used, the underlying logic considered, the validity of the results for any set or not and possible given constructions. Still, some observations need to be made. The epistemic state used in the works [YJW14, Yua17] is a belief state, which is a pair formed by a belief base and a set of core beliefs, the latter being logically closed within the base; so, in a general way, we considered as belief base. About [Fuh88], although the author worked on both types of input primacy, his main focus was on prioritized multiple revision, giving not only a construction but postulates and a (partial) representation theorem; for choice revision he only discussed some ideas and gave a possible construction. Regarding the assumption on the finiteness or not of the input, when the work is not specifically clear about [FKIS02, $\mathrm{KTF}^{+} 11$ ], we preferred to consider them as for finite inputs.

\subsection{Open Problems}

In this final section we list some open problems for this context as well. Looking at the approach developed in $\left[\mathrm{KTF}^{+} 11\right]$, the framework could be extended to preferences (both on the argumentation and on the belief revision sides) there could be an investigation about 


\begin{tabular}{|c|c|c|c|c|c|c|c|}
\hline \multirow{2}{*}{ Paper } & \multicolumn{3}{|c|}{ Epistemic Model } & \multirow{2}{*}{ Logic } & Type of Input & \multirow{2}{*}{ Construction } \\
\cline { 2 - 3 } \cline { 6 - 7 } & B. Set & B. Base & Poss. Worlds & & Finite & Infinite & \\
\hline \hline$[$ Fuh88] & $\mathrm{X}$ & & & Class. Propositional & & $\mathrm{X}$ & Partial Meet \\
\hline$[$ FKIS02] & & $\mathrm{X}$ & & Class. Propositional & $\mathrm{X}$ & & P. Meet and Kernel \\
\hline$[$ Del11] & $\mathrm{X}$ & & $\mathrm{X}$ & Class. Propositional & $\mathrm{X}$ & & Remaind. and F. Assign. \\
\hline$\left[\mathrm{KTF}^{+} 11\right]$ & & $\mathrm{X}$ & & Class. Propositional & $\mathrm{X}$ & & Deductive Argument. \\
\hline$[$ YJW14] & & $\mathrm{X}$ & & Class. Propositional & & $\mathrm{X}$ & Part. Meet and Kernel \\
\hline$[$ Yua17] & & $\mathrm{X}$ & & Class. Propositional & & $\mathrm{X}$ & P. Meet and Kernel \\
\hline$[$ Zha18] & & $\mathrm{X}$ & & Class. Propositional & $\mathrm{X}$ & & P. Meet (+ P. Expansion) \\
\hline$[$ Han17, Zha19] & $\mathrm{X}$ & & & Class. Propositional & $\mathrm{X}$ & & Desc. Rev. and M. Bel. Rel. \\
\hline
\end{tabular}

Table 4.1: Summary of the general characteristics of the different non-prioritized approaches. 
the connections with epistemic entrenchment. From [Del11], a possible extension of the work is to deal with sentences with different degrees of trustworthiness, and integrating such an implicit ordering on observations with the believability ordering implicit in the agent's ranking function on worlds.

Regarding Selective Revision, further studies on its generalization to the multiple case could be carried out, both for theories and belief bases; it will be the main focus of Chapter 6 where this generalization is explored taking into account different success possibilities. From semi-revision, a research opportunity is to explore how to define multiple semirevision from merge operators.

From [YJW14], a possible future work is the characterization of non-prioritized multiple revision in an unified way (without dividing it into two modules). From [Yua17], further exploration includes the definition of consolidation based on core beliefs and its relation with metabolic revision. For the choice revision operators defined in [Zha18] it remains to study and establish the differences and connections between these operators and the one based on Descriptor Revision from [Zha19]. The operation could be defined for inconsistent belief bases and for infinite inputs, as well. Choice could also be explored and constructed for belief bases without using contraction as an intermediate step (avoiding, thus, negation and disjunction of sentences), a topic that will be covered in Chapter 7 through Partial Meet and Kernel.

Finally, another possible future work it to study and analyze how to define a nonprioritized revision operation based on systems of spheres. 


\section{Chapter 5}

\section{Selective Base Revision}

As seen in Section 2.7.2, Selective Revision [FH99] came up as a third possibility for nonprioritized revision. The agent does not need to accept or reject a new belief completely, but may also accept part of the input. For this purpose, a weakening of the input sentence needs to be applied. This weakening is performed by a transformation function, to which the incoming information is submitted to perform an evaluation. Then the agent applies a traditional (prioritized) revision of its beliefs by the outcome of that function. The following figure summarizes the general behaviour of selective revision:

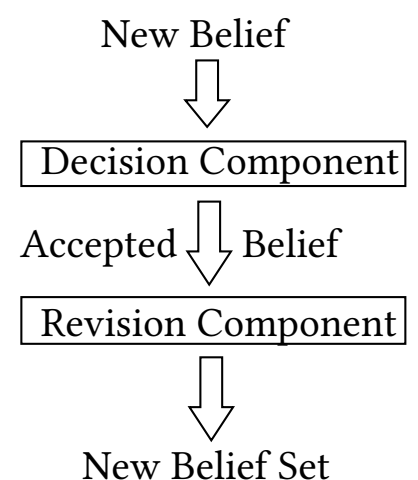

Selective revision was initially defined for sets of sentences closed under logical consequence. However, as stated in Chapter 1, in some scenarios, it is useful to work with belief bases, especially for computational purposes or when you have to deal with more realistic agents. In addition, the work in [FH99] was described for propositional logic, which makes the original operator not directly applicable to logics not closed under negation of sentences, such as Horn Logic and many Description Logics.

Therefore, in this chapter, we propose an extension of the original operator of Selective Revision to work with arbitrary sets (not necessarily closed under logical consequence) and not depend on negation of sentences. New operators are defined and axiomatically constructed. All the proofs were moved to Appendix A.

During the development of the material of this chapter, we found out that a research group from Universidade da Madeira (Portugal) was working on that as well. We joined 
some results in [RGW 20], and Garapa's previous studies were published in [Gar21].

\subsection{Properties}

We start by presenting a list of possible postulates for the operation. Let $\circledast$ be a selective base revision operator (defined properly further ahead), $B$ be a belief base and $\alpha, \beta$ sentences of $\mathcal{L}$.

(weak consistency) If $\alpha \forall \perp$, then $B \circledast \alpha \forall \perp$

(vacuity) If $B H \neg \alpha$, then $B \cup\{\alpha\} \subseteq B \circledast \alpha$

Weak consistency guarantees consistency of the final result as long as the input is consistent. Vacuity says that if $\alpha$ is not in conflict with $B$, then it will be fully incorporated. The next set of postulates are weaker versions of success:

(proxy success) There is a sentence $\beta$, such that $\beta \in B \circledast \alpha, \vdash \alpha \rightarrow \beta$ and $B \circledast \alpha=B \circledast \beta$

(weak proxy success) There is a sentence $\beta \in B \circledast \alpha$ such that $B \oplus \alpha=B \circledast \beta$

(stability) If $\alpha \in B$, then $\alpha \in B \circledast \alpha$

(uniform success) If for all subsets $B^{\prime} \subseteq B, B^{\prime} \cup\{\alpha\} \vdash \perp$ iff $B^{\prime} \cup\{\beta\} \vdash \perp$, then $\alpha \in B \circledast \alpha$ iff $\beta \in B \circledast \beta$

Proxy success states that the selective revision should accept and fully incorporate some part of the input information. Weak proxy success is a weaker version of proxy success that does not restrict $\beta$ to be implied by $\alpha$. Stability states that explicit beliefs of an agent (i.e. that are in the agent's belief base) should be in the outcome of a selective revision by that belief. Uniform success states that if two beliefs are inconsistent with exactly the same subsets of $B$, then one of them should be incorporated in the outcome of the selective revision by it iff the same thing happens with the other.

The following postulates are weaker versions of the postulates for base revision presented in Section 2.6.2.1:

(weak inclusion) If $\alpha \in B \circledast \alpha$, then $B \circledast \alpha \subseteq B \cup\{\alpha\}$

(weak uniformity) If $\alpha \in B \circledast \alpha$ and for all subsets $B^{\prime}$ of $B$ it holds that $B^{\prime} \cup\{\alpha\} \vdash \perp$ iff $B^{\prime} \cup\{\beta\} \vdash \perp$, then $B \cap(B \circledast \alpha)=B \cap(B \circledast \beta)$

(weak relevance) If $\alpha \in B \circledast \alpha, \beta \in B$ and $\beta \notin B \circledast \alpha$, then there is some $B^{\prime}$ such that $B \circledast \alpha \subseteq B^{\prime} \subseteq B \cup\{\alpha\}, B^{\prime} H \perp$ but $B^{\prime} \cup\{\beta\} \vdash \perp$

(weak core-retainment) If $\alpha \in B \circledast \alpha, \beta \in B$ and $\beta \notin B \circledast \alpha$, then there is some $B^{\prime} \subseteq B$ such that $B^{\prime} \forall \neg \alpha$ and $B^{\prime} \cup\{\beta\} \vdash \neg \alpha$

The weakening resides in the fact that they are preconditioned by $\alpha \in B \circledast \alpha$. Informally, this means that, if a sentence is in the outcome of a selective revision by it, then that outcome behaves as one coming from a standard revision. 
It is important to highlight that, in this chapter and in the next ones, we follow the traditional nomenclature of the literature that calls a postulate weak when there is a precondition for it, but this term is not related to any possible deficiency or weakness of the operation.

\subsection{Constructing the Operation}

The operation of selective base revision can be constructed as follows:

Definition 90. Let $B$ be a belief base, * be a singleton base revision operator on $B$ (Definitions 5 and 11) and $f$ be a function from $(\mathcal{L} \times \mathcal{P}(\mathcal{L}))$ to $\mathcal{L}$. The selective base revision $\circledast$, based on * and $f$, is the operation such that for all sentences $\alpha$ :

$$
B \circledast \alpha=B * f(\alpha)^{1}
$$

$f$ is the transformation function on which $\circledast$ is based.

We now present a list of properties that the transformation function may be expected to satisfy:

$$
\begin{aligned}
& \text { (implication) } \vdash \alpha \rightarrow f(\alpha) \\
& \text { (idempotence) } f(f(\alpha))=f(\alpha)
\end{aligned}
$$

(consistency preservation) If $\forall \neg \alpha$, then $\forall \neg f(\alpha)$

(weak maximality) If $B H \neg \alpha$, then $f(\alpha)=\alpha$

(lower boundary) If $\alpha \in B$, then $f(\alpha)=\alpha$

(uniform identity) If for all $B^{\prime} \subseteq B, B^{\prime} \cup\{\alpha\} \vdash \perp$ iff $B^{\prime} \cup\{\beta\} \vdash \perp$, then $f(\alpha)=\alpha$ iff $f(\beta)=\beta$

The first four properties were already recalled in Section 2.7.2 or result from those by adapting the namesake property to the belief base context. We note if a transformation function satisfies implication, then it also satisfies consistency preservation. Lower boundary states that an agent's explicit belief should be in the outcome of the selective revision by it. Uniform identity states that if two sentences are inconsistent with exactly the same subsets of $B$, then one of them should be fully accepted and incorporated iff the same thing happens regarding the other one.

The following observation shows how properties of the transformation function (eventually combined with postulates of base revision) give rise to selective revision postulates.

Observation 12. Let $B$ be a belief base, * be a singleton base revision operator on $B$ that satisfies success, inclusion and consistency and $f$ be a transformation function. Let $\circledast$ be the selective base revision operator on $B$ based on * and $f$. Then:

\footnotetext{
${ }^{1}$ Although we write $f(\alpha)$, actually the transformation function is $f_{B}(\alpha)$ since it also depends on $B$. We will use $f(\alpha)$ for short.
} 
1. Iff satisfies lower boundary, then $\circledast$ satisfies stability.

2. Iff satisfies lower boundary, then $\circledast$ satisfies weak inclusion.

3. If $f$ satisfies consistency preservation, then $\circledast$ satisfy consistency.

4. If $f$ satisfies weak maximality and * satisfies vacuity, then $\circledast$ satisfies vacuity.

5. Iff satisfies idempotence, then $\circledast$ satisfies weak proxy success.

6. Iff satisfies idempotence and implication, then $\circledast$ satisfies proxy success.

7. If * satisfies relevance and $f$ satisfies lower boundary, then $\circledast$ satisfies weak relevance.

8. If * satisfies core-retainment and $f$ satisfies lower boundary, then $\circledast$ satisfies weak core-retainment.

9. If $f$ satisfies uniform identity and lower boundary, then $\circledast$ satisfies uniform success.

10. Iff satisfies uniform identity and lower boundary and * satisfies uniformity, then $\circledast$ satisfies weak uniformity.

\subsection{Representation Results}

Now we present axiomatic characterizations for two different classes of selective base revision. More precisely, we give representation theorems based on partial meet revisions and kernel revisions, with two variants for each.

Theorem 51. Let $B$ be a belief base, and $\otimes$ be an operator on $B$. Then the following pair of conditions are equivalent:

(a) $\circledast$ satisfies weak inclusion, weak consistency, weak uniformity, proxy success, stability, uniform success and weak relevance.

(b) There exists a partial meet base revision operator * for $B$ and a transformation function $f$ that satisfies lower boundary, consistency preservation, idempotence, implication, uniform identity and such that $B \circledast \alpha=B * f(\alpha)$, for all $\alpha$.

The next theorem is a slight variant of the former, substituting proxy success by weak proxy success in the postulates for $\circledast$. For this purpose, $f$ is not required to satisfy implication.

Theorem 52. Let $B$ be a belief base, and $\otimes$ be an operator on $B$. Then the following pair of conditions are equivalent:

(a) $\circledast$ satisfies weak inclusion, consistency, weak uniformity, weak proxy success, stability, uniform success and weak relevance.

(b) There exists a partial meet base revision operator * for $B$ and a transformation function $f$ that satisfies lower boundary, consistency preservation, idempotence and uniform identity and such that $B \circledast \alpha=B * f(\alpha)$, for all $\alpha$.

Now for kernel revision: 
Theorem 53. Let $B$ be a belief base, and $\circledast$ be an operator on $B$. Then the following pair of conditions are equivalent:

(a) $\circledast$ satisfies weak inclusion, weak consistency, weak uniformity, proxy success, stability, uniform success and weak core-retainment.

(b) There exists a kernel base revision operator * for $B$ and a transformation function $f$ that satisfies lower boundary, consistency preservation, idempotence, implication, uniform identity and such that $B \circledast \alpha=B * f(\alpha)$, for all $\alpha$.

To conclude, the weak proxy success version, whose proof is very similar to that of Theorem 53, as explained for Theorem 52:

Theorem 54. Let $B$ be a belief base, and $\circledast$ be an operator on $B$. Then the following pair of conditions are equivalent:

(a) $\circledast$ satisfies weak inclusion, weak consistency, weak uniformity, weak proxy success, stability, uniform success and weak core-retainment.

(b) There exists a kernel base revision operator * for $B$ and a transformation function $f$ that satisfies lower boundary, consistency preservation, idempotence and uniform identity and such that $B \circledast \alpha=B * f(\alpha)$, for all $\alpha$.

The main difference between these postulates is on the success postulates. Theorems 51 and 53 look at the operations in which $f(\alpha)$ does not return more information than what is expressed in $\alpha$. On the other hand, Theorems 52 and 54 embrace very general operations which do not demand $f(\alpha)$ to be derived from $\alpha$.

\subsection{Negation Free Selective Base Revision}

Keeping in mind the importance of providing a theory suitable for logics not closed under negation of sentences, as explained in Section 2.6.5, in this section, we are going to show how Selective Revision can be extended taking this restriction into account.

\subsubsection{Properties}

Most of the postulates proposed in Section 5.1 for bases stay the same. The postulates that need to be adapted in order to not use negation are vacuity and weak coreretainment:

(vacuity) If $B \cup\{\alpha\} \forall \perp$, then $B \cup\{\alpha\} \subseteq B \circledast \alpha$

(weak core-retainment) If $\alpha \in B \circledast \alpha, \beta \in B$ and $\beta \notin B \circledast \alpha$, then there is some $B^{\prime} \subseteq B$ such that $B^{\prime} \cup \alpha \forall \perp$ and $B^{\prime} \cup\{\alpha, \beta\} \vdash \perp$

In addition, in order to characterize this operation, we need a new postulate that is a weaker version of pre-expansion. The original idea of this postulate is that the consecutive act of expansion by $\alpha$ and revision by $\alpha$ should bring the same outcome as that of only performing the revision. In our context, due to the partial acceptance nature of selective revision, we cannot always guarantee that the successive performance of expansion by $\alpha$ and selective revision by $\alpha$ has the same outcome as that of only performing the selective 
revision. Nevertheless, if $\alpha$ is in the final result of a selective revision by $\alpha$, we can ensure the referred property:

(weak pre-expansion) If $\alpha \in B \circledast \alpha$, then $(B \cup\{\alpha\}) \circledast \alpha=B \circledast \alpha$

\subsubsection{Constructing the Operation}

The definition of the operation is equal to the one for bases in the previous section, except for the fact that, this time, the base revision operators used are the negation free ones (Theorems 5 and 6).

The only difference is on some of the properties for the transformation function, also to eliminate negation of sentences:

(consistency preservation) If $\alpha \forall \perp$, then $f(\alpha) \forall \perp$

(weak maximality) If $B \cup\{\alpha\} \forall \perp$, then $f(\alpha)=\alpha$

Observation 12 is still valid here. Its proof just needs to be adapted substituting $\sharp \neg \alpha$ by $\alpha H \perp$ and $B H \neg \alpha$ by $B \cup\{\alpha\} \forall \perp$.

\subsubsection{Representation Results}

The following representation theorems have been obtained for two classes of negation free selective base revision:

Theorem 55. Let $B$ be a belief base, and $\circledast$ be an operator on $B$. Then the following conditions are equivalent:

(a) ® satisfies weak inclusion, weak consistency, weak uniformity, proxy success, stability, uniform success, weak relevance and weak pre-expansion.

(b) There exists a negation free external partial meet base revision operator * for $B$ and a transformation function $f$ that satisfies lower boundary, consistency preservation, idempotence, implication, uniform identity and such that $B \circledast \alpha=B * f(\alpha)$, for all $\alpha$.

Theorem 56. Let $B$ be a belief base, and $\circledast$ be an operator on $B$. Then the following conditions are equivalent:

(a) $\circledast$ satisfies weak inclusion, weak consistency, weak uniformity, weak proxy success, stability, uniform success, weak relevance and weak pre-expansion.

(b) There exists a negation free external partial meet base revision operator * for $B$ and a transformation function $f$ that satisfies lower boundary, consistency preservation, idempotence, uniform identity and such that $B \circledast \alpha=B * f(\alpha)$, for all $\alpha$.

Theorem 57. Let $B$ be a belief base, and $\circledast$ be an operator on $B$. Then the following conditions are equivalent:

(a) $\circledast$ satisfies weak inclusion, weak consistency, weak uniformity, proxy success, stability, uniform success, weak core-retainment and weak pre-expansion. 
5.5 | RELATED WORK

(b) There exists a negation free external kernel base revision operator * for $B$ and a transformation function $f$ that satisfies lower boundary, consistency preservation, idempotence, implication, uniform identity and such that $B \circledast \alpha=B * f(\alpha)$, for all $\alpha$.

Theorem 58. Let $B$ be a belief base, and $\circledast$ be an operator on $B$. Then the following conditions are equivalent:

(a) $\circledast$ satisfies weak inclusion, weak consistency, weak uniformity, weak proxy success, stability, uniform success, weak core-retainment and weak pre-expansion.

(b) There exists a negation free external kernel base revision operator * for B and a transformation function $f$ that satisfies lower boundary, consistency preservation, idempotence, uniform identity and such that $B \circledast \alpha=B * f(\alpha)$, for all $\alpha$.

The proofs for these last three theorems are very similar to the ones in the previous section. Theorem 56 is just a version for weak proxy success of Theorem 55, as well as occurred between Theorems 51 and 52. Concerning Theorems 57 and 58, the proofs for them are very similar to the ones for Theorems 53 and 54, respectively, except for the weak pre-expansion part, which can be obtained from Theorem 55.

\subsection{Related Work}

There is broad literature about non-prioritized revision operations for singleton inputs. Screened Revision [Mak97], for example, explores the context in which an agent, in addition to its set of beliefs $K$, makes use of a set of core beliefs $A$ that cannot be retracted. Then, an input sentence is accepted for revision only if it is consistent with $K \cap A$. In a slightly different approach, Credibility-limited Revision [HFCF01] considers that there is a set $\mathcal{C}$ of credible sentences and an input sentence $\alpha$ is accepted for revision only if $\alpha \in \mathcal{C}$. Semi-revision [Han97], as explained in Section 2.7.1, is defined as an operation firts accepts the new belief and, then, performs a consolidation step.

Although screened revision and credibility-limited revision are inserted in the decision + revision variety of non-prioritized revision as well as selective base revision, they were defined to work with belief sets instead of belief bases. In addition, the decision step of selective revision does not depend on a set of core beliefs or credible sentences, but on a transformation function $f$. As a final remark, the operation proposed in this chapter was also extended to the context of logics not closed under negation of sentences. 



\section{Chapter 6}

\section{Multiple Selective Revision}

In the previous chapter, we extended the original Selective Revision operation to work with belief bases. As well as in AGM revision, selective revision was also initially defined for single sentences as inputs. Nevertheless, in many situations, an agent receives not only a single new belief but a set of them and has to make decisions on the face of it. One example of the importance of studying contexts where an agent has to deal simultaneously with multiple information is the scenario described in [PSLS16] and [SPS18], in which the authors study the application of perception filters in agents. As already discussed in the Introduction, pre-processing information may advance an agent's performance. In order to illustrate the idea, consider the following example.

Example 2. Imagine that three robots (including a coordinator $C$ ) are in a house that needs to be cleaned up. Initially, $C$ knows that the bedroom is organized but full of dust, the bathroom is flooded, the kitchen is full of food scraps, but the living room is neat. Before starting the job, robots $A$ and $B$ collect some perceptions. After some time, they jointly report to $C$. A said that (i) the beds in the bedroom need to be made and that (ii) there is a silver tap in the bathroom that is open. B told that (iii) a dinosaur broke a vase in the living room and that (iv) the kitchen is clean and organized. Before revising its beliefs, $C$ applies a filter, which accepts (i) and (ii), except for the information about the tap's material, as there is no silver in that country. From (iii), the filter accepts that there is a broken vase in the living room but rejects the dinosaur part. (iv) is fully rejected. After that, $C$ performs a prioritized multiple revision of its beliefs.

In this chapter, then, we propose a generalization of the operation of Selective Revision. In the next section, we will generalize the operation to the multiple case when dealing with belief sets, while in Section 6.2 the focus will be on belief bases. All the proofs were moved to Appendix A.

\subsection{Multiple Selective Theory Revision}

In this section, we will show how to define and axiomatically characterize multiple selective revision for belief sets (theories). 


\subsubsection{Properties}

Some of the postulates for Multiple Theory Revision (Section 3.1.5) remain the same: closure, inclusion, weak consistency, uniformity and vacuity.

As we already discussed, due to the non-prioritized nature of Selective Revision, the success postulate is not suitable in this context. So we generalized its two weaker versions presented in [FH99] to consider sets of sentences as input. The same was done for consistent expansion and a weaker version of relevance.

Let $K$ be a belief set, $A$ and $C$ be sets of sentences and $\odot$ be a binary multiple selective revision operator (defined properly further ahead) that takes a belief set and a set of sentences as input. We propose the following reasonable postulates for multiple selective theory revision:

(choice success) There is a set $B$ such that $B \subseteq C n(K \odot A), B \subseteq A$ and $K \odot A=K \odot B$

(proxy success) There is a set $B$ such that $B \subseteq C n(K \odot A), B \subseteq C n(A)$ and $K \odot A=K \odot B$

(weak proxy success) There is a set $B$ such that $B \subseteq C n(K \odot A)$ and $K \odot A=K \odot B$

(conditional success) If $A \backslash K \subseteq K \odot A$, then $A \subseteq K \odot A$

(uniform success) If for every $K^{\prime} \subseteq K$ it holds that $K^{\prime} \cup A$ is inconsistent iff $K^{\prime} \cup C$ is inconsistent, then $A \subseteq K \odot A$ iff $C \subseteq K \odot C$

Choice success states that the selective revision should incorporate a subset of the input set. Proxy success establishes that the selective revision should accept some of the input's logical consequences, while weak proxy success is a weaker version of it not requiring $B \cap C n(A) \neq \varnothing$. Conditional success guarantees that if the difference between $A$ and $K$ is part of the final result, then so is the whole $A$, which means that, if $A \backslash K$ is accepted, the intersection between them will not be rejected. Uniform success shows that, if two sets are inconsistent with the same subsets of the original belief set, then one of the input sets should be absorbed in the multiple selective revision by it iff the same happens to the other one.

(closure) $K \odot A=C n(K \odot A)$

(inclusion) $K \odot A \subseteq C n(K \cup A)$

(uniformity) If for every $K^{\prime} \subseteq K$ it holds that $K^{\prime} \cup A$ is inconsistent iff $K^{\prime} \cup C$ is inconsistent, then $K \odot A=K \odot C$

(vacuity) If $K \cup A$ is consistent, then $K \odot A=C n(K \cup A)$

(stability) If $A \subseteq K$, then $A \subseteq K \odot A$.

Closure guarantees the logical closure of the result and inclusion establishes the limits of it. Uniformity shows that if two sets are inconsistent with the same subsets of the original belief set, then the outcome of the multiple selective theory revision by each of them should be the same. While vacuity sets what happens when there is no inconsistency between the belief set and the input, stability brings that if the input set is already part of the agent's beliefs, it should be kept by the selective revision. 
(consistency) $K \odot A$ is consistent

(weak consistency) If $A$ is consistent, then $K \odot A$ is consistent

(consistent expansion) If $K \nsubseteq K \odot A$, then $K \cup(K \odot A)$ is inconsistent

(weak relevance) If $A \subseteq K \odot A, \beta \in K$ and $\beta \notin K \odot A$, then there is some $K^{\prime}$ such that $(K \odot A) \cap K \subseteq K^{\prime} \subseteq K, K^{\prime} \cup A$ is consistent but $K^{\prime} \cup\{\beta\} \cup A$ is inconsistent

Consistency guarantees an always consistent result, while weak consistency demands a consistent input for that. Consistent expansion and weak relevance express the idea that nothing is given up from the original set unless it leads the new belief set to consistency (the last one preconditioned by $A$ in the final result).

Except for weak consistency and consistent expansion (already presented in [ $\left.\mathrm{KTF}^{+} 11\right]$ ) and also for the ones that came from Multiple Package Theory Revision, the other postulates are new. Uniform success and conditional success are straightforward generalizations of postulates for Selective Base Revision $\left[\mathrm{RGW}^{+} 20\right]$, that appeared in Chapter 5.

\subsubsection{Constructing the Operation}

The intended operator can be constructed as follows:

Definition 91. Let $K$ be a belief set, ${ }_{p}$ be a multiple package theory revision for $K$ and $f$ be a function from $(\mathcal{P}(\mathcal{L}) \times \mathcal{P}(\mathcal{L}))$ to $\mathcal{P}(\mathcal{L})$. The multiple selective theory revision $\odot$, based on ${ }^{*} p$ and $f$, is the operation such that for all sets $A$ :

$$
K \odot A=K{ }_{p} f(A)^{1}
$$

$f$ is the transformation function on which $\odot$ is based.

Selective revision becomes, then, a particular case of this new operator $\odot$. One can question why a multiple revision operator is needed to construct the operation, perhaps suggesting a sequence of singleton input revisions by the elements of $f(A)$. Nonetheless, as already discussed in the Introduction, multiple revision is different from iterated revision since the sequence in which you process the sentences can result in different outcomes. Thus, we want here to treat all the sentences with equal priority, processing them simultaneously.

The following is a list of properties that the transformation function may satisfy:

(choice) $f(A) \subseteq A$

(implication) $f(A) \subseteq C n(A)$

(weak implication) If $K \cup A \forall \perp$, then $f(A) \subseteq C n(A)$

(lower boundary) if $A \subseteq K$, then $C n(f(A))=C n(A)$

(idempotence) $C n(f(f(A)))=C n(f(A))$

(consistency preservation) If $A H \perp$, then $f(A) \forall \perp$

\footnotetext{
${ }^{1}$ Although we write $f(A)$, actually the transformation function is $f_{K}(A)$ since it also depends on $K$. We will use $f(A)$ for short.
} 
(consistency) $f(A) \Vdash \perp$

(maximality) $C n(f(A))=C n(A)$

(weak maximality) If $K \cup A \forall \perp$, then $C n(f(A))=C n(A)$

(uniformity) If for every $K^{\prime} \subseteq K$ it holds that $K^{\prime} \cup A$ is inconsistent iff $K^{\prime} \cup C$ is inconsistent, then $K^{\prime} \cup f(A)$ is inconsistent iff $K^{\prime} \cup f(C)$ is inconsistent

(uniform identity) If for every $K^{\prime} \subseteq K$ it holds that $K^{\prime} \cup A$ is inconsistent iff $K^{\prime} \cup C$ is inconsistent, then $C n(f(A))=C n(A)$ iff $C n(f(C))=C n(C)$

(conditional maximality) If $A \backslash K \subseteq C n(f(A))$, then $C n(f(A))=C n(A)$.

Choice sets the transformation function to simply choose a subset of the input set $A$. Implication allows the function to choose from the logical consequences of the input, while weak implication restricts to the the consequences of the input only if the input is consistent with the previous beliefs. Lower boundary states that if an input $A$ is already part of the previous beliefs, then $f(A)$ and $A$ have the same logical consequences. While consistency preservation demands consistency from $f(A)$ only if $A$ is consistent, consistency always guarantees a consistent $f(A)$. Maximality states that $f(A)$ and $A$ are logically equivalent, while weak maximality states a precondition for that. Uniformity guarantees a coherent behavior of $f$ when different inputs are inconsistent with the same subsets of $K$. Uniform identity is a version of uniformity for $f$ and conditional maximality states that if the difference between $A$ and $K$ is implied by the transformation function, then actually $A$ and $f(A)$ are logically equivalent. Choice, consistency preservation, consistency and extensionality had already been suggested in $\left[\mathrm{KTF}^{+} 11\right]$; the others are new. The observation below establishes some links between the properties for $f$ and the postulates for $\odot$ :

Observation 13. Let $K$ be a belief set in a language $\mathcal{L}$, * ${ }^{*}$ be a multiple package partial meet theory revision operator for $K$ that satisfies the six postulates referred in Observation 7 , and $f$ be a transformation function. Let $\odot$ be the multiple selective theory revision function on $K$ based on $*_{p}$ and $f$. Then $\odot$ satisfies closure and consistent expansion. In addition, iff satisfies:

1. weak implication, then $\odot$ satisfies inclusion.

2. consistency, then $\odot$ satisfies consistency.

3. maximality, then $\odot$ satisfies success.

4. implication, then $\odot$ satisfies weak consistency.

The following observation clarifies an important property: it guarantees that $f(A)$ does not reject anything of $A$ given that $A$ is in the final result.

Observation 14. Let $K$ be a belief set, * p be a multiple package theory revision operator on $K$ that satisfies *-inclusion, and $f$ be a transformation function. Let $\odot$ be the multiple selective theory revision operator on $K$ based on ${ }_{p}$ and $f$. If $A \subseteq K \odot A$ and $f$ satisfies conditional maximality, then $C n(f(A))=C n(A)$. 


\subsubsection{Representation Results}

The following representation theorems have been obtained for three classes of multiple selective theory revision functions. Once more, the differences between them lie on the success postulates, which are directly influenced by the properties of the transformation function.

Theorem 59. Let $\mathcal{L}$ be a finite language, $K$ be a belief set in $\mathcal{L}$, and $\odot$ be an operator on $K$. The following conditions are equivalent:

1. $\odot$ satisfies closure, weak proxy success, inclusion, vacuity, weak consistency, uniformity, uniform success, conditional success, stability, and weak relevance.

2. There exists a multiple package partial meet theory revision ${ }^{*}$ for $K$ that satisfies the six postulates referred in Observation 7, and a transformation function $f$ that satisfies uniformity, uniform identity, lower boundary, conditional maximality, consistency preservation, weak maximality and idempotence, such that $K \odot A=K{ }^{*} f(A)$ for all A.

Theorem 60. Let $\mathcal{L}$ be a finite language, $K$ be a belief set in $\mathcal{L}$, and $\odot$ be an operator on $K$. Then the following conditions are equivalent:

1. $\odot$ satisfies closure, proxy success, inclusion, vacuity, weak consistency, uniformity, uniform success, conditional success, stability, and weak relevance.

2. There exists a multiple package partial meet theory revision * for $K$ that satisfies the six postulates referred in Observation 7, and a transformation function $f$ that satisfies uniformity, uniform identity, lower boundary, conditional maximality, consistency preservation, weak maximality, idempotence and implication, such that $K \odot A=K{ }^{*} p$ $f(A)$ for all $A$.

Theorem 61. Let $\mathcal{L}$ be a finite language, $K$ be a belief set in $\mathcal{L}$, and $\odot$ be an operator on $K$. Then the following conditions are equivalent:

1. $\odot$ satisfies closure, choice success, inclusion, vacuity, weak consistency, uniformity, uniform success, conditional success, stability, and weak relevance.

2. There exists a multiple package partial meet theory revision * for $K$ that satisfies the six postulates referred in Observation 7, and a transformation function $f$ that satisfies uniformity, uniform identity, lower boundary, conditional maximality, consistency preservation, weak maximality, idempotence and choice, such that $K \odot A=K{ }_{{ }_{p}} f(A)$ for all $A$.

Theorem 59 embraces very general operations which do not demand $f(A)$ to be derived from $A$. Theorem 60 looks at the operations in which $f(A)$ does not return more information than what is expressed in $A$. Finally, Theorem 61, although more restrictive, represents the most intuitive procedure when $f(A)$ selects a subset of $A$. 


\subsection{Multiple Selective Base Revision}

As an alternative to the approach proposed in the previous section, we now show how to define and axiomatically characterize multiple selective revision for belief bases.

\subsubsection{Properties}

In comparison to what was defined for belief sets, we have the exclusion of the closure postulate, an exchange of extensionality for uniformity, adaptations in inclusion and vacuity (removing the logical closure) and new versions for the success and inclusion postulates (due to the context of belief bases). From Multiple Internal Base Revision (Theorem 15), inclusion and weak consistency remain the same.

Let $B$ be a belief set, $A$ and $C$ be sets of sentences and $\odot$ be a binary multiple selective revision operator (defined properly further ahead). We bring the following reasonable postulates for multiple selective base revision:

(choice success) There is a set $C$ such that $C \subseteq B \odot A, C \subseteq A$ and $B \odot A=B \odot C$.

(proxy success) There is a set $C$ such that $C \subseteq B \odot A, C \subseteq C n(A)$ and $B \odot A=B \odot C$.

(weak proxy success) There is a set $C$ such that $C \subseteq B \odot A$ and $B \odot A=B \odot C$.

(conditional success) If $A \backslash B \subseteq B \odot A$, then $A \subseteq B \odot A$.

(uniform success) If for all subsets $B^{\prime} \subseteq B, B^{\prime} \cup A \vdash \perp$ iff $B^{\prime} \cup C \vdash \perp$, then $A \subseteq B \odot A$ iff $C \subseteq B \odot C$.

Conditional success guarantees that if the difference between $A$ and $B$ is part of the final result, then so is the whole $A$, which means that, if $A \backslash B$ is accepted, then the intersection between them will not be rejected. Uniform success says that if two sets are inconsistent with exactly the same subsets of $B$, then one of them should be absorbed in the selective revision by it iff the same happens to the other one.

(vacuity) If $B \cup A \nvdash \perp$, then $B \odot A=B \cup A$

(consistency) $B \odot A \nvdash \perp$

(weak consistency) If $A \forall \perp$, then $B \odot A \forall \perp$

(inclusion) $B \odot A \subseteq B \cup A$

(weak inclusion) $B \odot A \subseteq B \cup C n(A)$

(very weak inclusion) $B \odot A \subseteq C n(B \cup A)$

(conditional inclusion) If $A \subseteq B \odot A$, then $B \odot A \subseteq B \cup A$.

(weak relevance) If $A \subseteq B \odot A, \beta \in B$ and $\beta \notin B \odot A$, then there is some $B^{\prime}$ such that $B \odot A \subseteq B^{\prime} \subseteq B \cup A, B^{\prime} H \perp$ but $B^{\prime} \cup\{\beta\} \vdash \perp$.

(conditional uniformity) If $A \subseteq B \odot A$ and for all subsets $B^{\prime}$ of $B$ it holds that $B^{\prime} \cup A \vdash \perp$ iff $B^{\prime} \cup C \vdash \perp$, then $B \cap(B \odot A)=B \cap(B \odot C)$. 
Weak inclusion shows that the selective revision of $B$ by $A$ is contained in the union of $B$ and the logical consequences of $A$, while very weak inclusion says that it is contained in the logical consequences of the union of $B$ and $A$. Conditional inclusion is a weakening of the traditional inclusion postulate for multiple by preconditioning $A$ in the final result. For conditional uniformity, if $A$ is in the outcome and two consistent sets are inconsistent with the same subsets of the original base, then the respective retained sentences of $B$ should be identical. The other postulates' intuition is the same for Multiple Selective Theory Revision (Section 6.1.1).

Except for the new postulates choice success, conditional success, weak inclusion and very weak inclusion, the other ones are straightforward generalizations of postulates for Selective Base Revision [RGW $\left.{ }^{+} 20\right]$.

\subsubsection{Constructing the Operation}

The intended operator can be constructed as follows:

Definition 92. Let $B$ be a belief base, ${ }^{\circ}$ be multiple package internal base revision for $B$ and $f$ be a function from $(\mathcal{P}(\mathcal{L}) \times \mathcal{P}(\mathcal{L}))$ to $\mathcal{P}(\mathcal{L})$. The multiple selective base revision $\odot$, based on $*_{p}$ and $f$, is the operation such that for every finite set $A$ :

$$
B \odot A=B *_{p} f(A)^{2}
$$

$f$ is the transformation function on which $\odot$ is based.

Similarly to the previous section, selective base revision becomes a particular case of this new operator $\odot$.

From Multiple Selective Theory Revision, some potential properties for $f$ remain the same: choice, implication, weak implication, consistency and consistency preservation. Some others needed to be adapted for belief bases. Extensionality was substituted by uniform identity, which has a similar intuition.

(maximality) $f(A)=A$

(weak maximality) If $B \cup A \forall \perp$, then $f(A)=A$

(conditional maximality) if $A \backslash B \subseteq f(A)$, then $f(A)=A$

(idempotence) $f(f(A))=f(A)$

(uniform identity) if for all $B^{\prime} \subseteq B, B^{\prime} \cup A \vdash \perp$ iff $B^{\prime} \cup C \vdash \perp$, then $f(A)=A$ iff $f(C)=C$.

Uniform identity is a version of uniformity for $f$. Conditional maximality states that if the difference between $A$ and $B$ is chosen by the transformation function, then actually the function chose the whole $A$. Maximality and weak maximality were suggested in $\left[\mathrm{KTF}^{+} 11\right]$.

\footnotetext{
${ }^{2}$ Although we write $f(A)$, actually the transformation function is $f_{B}(A)$ since it also depends on $B$. We will use $f(A)$ for short.
} 
Observation 15. Let $B$ be a belief base, * ${ }_{p}$ be a multiple package internal partial meet base revision operator on $B$ that satisfies the postulates described in Theorem 15 and $f$ be $a$ transformation function. Let $\odot$ be the multiple selective base revision operator on $B$ based on ${ }_{p}$ and $f$. Then iff satisfies:

1. weak implication, then $\odot$ satisfies very weak inclusion.

2. consistency preservation, then $\odot$ satisfies weak consistency.

3. implication, then $\odot$ satisfies weak inclusion and weak consistency.

4. choice, then $\odot$ satisfies inclusion.

5. weak maximality, then $\odot$ satisfies very weak inclusion and vacuity.

Similarly to what happens with belief sets, the following observation demonstrates that $f(A)$ does not reject anything of $A$ given that $A$ is in the final result.

Observation 16. Let $B$ be a belief base, ${ }_{p}$ be a multiple package internal base revision operator on $B$ that satisfies *-inclusion and $f$ be a transformation function. Let $\odot$ be the multiple selective base revision operator on $B$ based on ${ }^{*} p$ and $f$. If $A \subseteq B \odot A$ and $f$ satisfies conditional maximality, then $f(A)=A$.

\subsubsection{Representation Results}

The following representation theorems have been obtained for three classes of multiple selective base revision functions. The first one involves the weakest version of success:

Theorem 62. Let $B$ be a belief base in $\mathcal{L}$, and $\odot$ be an operator on $B$. Then the following conditions are equivalent:

1. $\odot$ satisfies conditional inclusion, weak consistency, conditional uniformity, weak proxy success, conditional success, uniform success and weak relevance.

2. There exists a multiple package partial meet base revision $*_{p}$ for $B$ that satisfies the postulates described in Theorem 15 and a transformation function $f$ that satisfies conditional maximality, consistency preservation, idempotence, uniform identity and such that $B \odot A=B{ }_{p} f(A)$, for every finite set $A$.

To conclude, the proxy success and choice success versions:

Theorem 63. Let $B$ be a belief base in $\mathcal{L}$, and $\odot$ be an operator on $B$. Then the following conditions are equivalent:

1. $\odot$ satisfies conditional inclusion, weak consistency, conditional uniformity, proxy success, conditional success, uniform success and weak relevance.

2. There exists a multiple package partial meet base revision $*_{p}$ for $B$ that satisfies the postulates described in Theorem 15 and a transformation function $f$ that satisfies conditional maximality, consistency preservation, idempotence, implication, uniform identity and such that $B \odot A=B{ }_{p} f(A)$, for every finite set $A$. 
6.3 | RELATED WORK

Theorem 64. Let $B$ be a belief base in $\mathcal{L}$, and $\odot$ be an operator on $B$. Then the following conditions are equivalent:

1. $\odot$ satisfies inclusion, weak consistency, conditional uniformity, choice success, conditional success, uniform success and weak relevance.

2. There exists a multiple package partial meet base revision ${ }^{*}$ for $B$ that satisfies the postulates described in Theorem 15 and a transformation function $f$ that satisfies conditional maximality, consistency preservation, idempotence, choice, uniform identity and such that $B \odot A=B{ }^{*} f(A)$, for every finite set $A$.

The intuition behind these theorems are pretty much the same as for theories. Theorem 62 allows very general operations which do not demand $f(A)$ to be derived from $A$. Theorem 63 refers to the operations in which $f(A)$ is limited to $A$ and its logical consequences. Finally, Theorem 64 represents the most restrictive ones since $f(A)$ is limited to the subsets of A.

\subsubsection{Example}

In this section, we return to the example given at the beginning of this chapter to show a possible application of the operators for bases defined here.

Example 3. (Example 2 revisited) Consider a representation in propositional logic for $C$ 's beliefs: the bedroom is organized $(\alpha)$ but full of dust $(\varphi)$, the bathroom is flooded $(\beta)$, the kitchen is full of food scraps $(\lambda)$, and the living room is neat $(\phi)$. In addition, a clean kitchen $(\psi)$ is not consistent with food scraps $(\lambda \rightarrow \neg \psi)$, and a broken object in the living room $(\delta)$ makes it not neat $(\delta \rightarrow \neg \phi)$. The other robots tell that the beds need to be made $(\neg \alpha)$, there is a silver tap in the bathroom ( $\omega)$ that is open $(\mu$, thus $\mu \wedge \omega)$, there is a broken vase in the living room $(\delta)$ because of a dinosaur that has entered there $(\theta$, thus $\theta \wedge \delta)$ and the kitchen is clean $(\psi)$.

We are going to represent the filter by an $f$ that satisfies implication, which means that it accepts the logical consequences of the input. Then the filter works as follows: $f(\{\neg \alpha, \mu \wedge$ $\omega, \theta \wedge \delta, \psi\})=\{\neg \alpha, \mu, \delta\}$.

After that, a prioritized revision is applied: $B \odot A=\{\alpha, \varphi, \beta, \lambda, \lambda \rightarrow \neg \psi, \phi, \delta \rightarrow \neg \phi\}{ }^{*} p$ $\{\neg \alpha, \mu, \delta\}$. A possible final result could be $\{\neg \alpha, \varphi, \mu, \beta, \lambda, \lambda \rightarrow \neg \psi, \phi, \delta, \delta \rightarrow \neg \phi\}$.

\subsection{Related Work}

As presented in Chapter 4, there is broad literature about non-prioritized revision operations that accept sets of sentences as input. Comparing to approaches that, as well as selective revision, follow the decision + revision two-step method, we find, for example, the use of core beliefs. Evaluative Multiple Revision [YJW14] works with belief states formed by a belief base $B$ and a subset $A$ of it that is immune to revision. An input set is submitted to a pre-processing that classifies its sentences into two disjoint sets of plausible or implausible information (consistent or not with $A$ ). Both sets are considered in the revision process since $B$ can be initially inconsistent. Then, all implausible information has to be given up. Our approach developed in this chapter does not assume that there is a core or a set 
of credible sentences, and permits partial acceptance (including weakening), making the transformation function more general.

In $\left[\mathrm{KTF}^{+} 11\right]$ the authors proposed a concrete implementation of a transformation function using Deductive Argumentation [BH01] as the tool to evaluate the desirability of new information for a belief base. To allow a new belief to contain arguments, they worked with a multiple version of Selective Revision for bases. However, they explored a smaller set of properties, not working with different success/inclusion cases or relevance, for example. Also, the input sets considered in this chapter do not originate from argumentation, and we also brought a generalization for theories. 


\section{Chapter 7}

\section{Multiple Choice Revision}

In this chapter, we will propose an axiomatic characterization of the operation of Multiple Choice Revision, which will be explored from two sides. Firstly, a list of rationality postulates that establish the properties that the operation may satisfy. Secondly, two mathematical constructions that show how the operation can be built. Representation theorems, then, prove the equivalence between the constructions and a set of rationality postulates. All the proofs can be found in Appendix B.

In this proposal, we are going to apply a partial expansion operator but with a slight modification in its construction. First, we define a weak partial sum set $\bowtie_{\perp}$ that is identical to the one from Definition 57 except for the fact that if $A \vdash \perp$, then $B \bowtie_{\perp} A=\varnothing$. The weak partial expansion operator $\dot{+}_{\perp}$ is proposed as in Definition 60 but applying $\bowtie_{\perp}$. A representation result is given below:

Theorem 65. + is a weak partial expansion iff, for every sets $A, B$ and $C$, it satisfies + inclusion, $\dot{+}$-preservation, $\dot{+}$-coincidence and, in addition, the following postulate:

(;-weak success) If $A \neq \varnothing$ and $A \forall \perp$, then $A \cap(B+A) \neq \varnothing$.

$\dot{+}$ is consistency-preserving iff it in addition satisfies +-consistency.

\subsection{Properties}

Let $A, B$ and $C$ be belief bases and $*_{c}$ be a binary multiple revision operator that takes a belief base and a set of sentences as input. We bring the following properties as reasonable postulates for choice revision operations:

$$
\begin{aligned}
& \left(*_{c} \text {-inclusion) } B *_{c} A \subseteq B \cup A\right. \\
& \text { ( }{ }_{c} \text {-weak success) if } A \neq \varnothing \text { and } A \forall \perp \text {, then } A \cap\left(B *_{c} A\right) \neq \varnothing \\
& \text { (* }{ }_{c} \text {-vacuity 1) if } A=\varnothing \text { or } A \vdash \perp \text {, then } B{ }^{*}{ }_{c} A=B \\
& \left(*_{c} \text {-vacuity 2) if } A \cap B \neq \varnothing \text {, then } B *_{c} A=B\right. \\
& \left(*_{c} \text {-vacuity } 3\right) \text { if } B \cup A \forall \perp \text {, then } B *_{c} A=B \cup A
\end{aligned}
$$


$\left(*_{c}\right.$-vacuity 4) if $B \cup A \forall \perp$, then $B \subseteq B *_{c} A$

(* $*_{c}$-strong consistency) $B *_{c} A H \perp$

( ${ }_{c}$-weak consistency) if $A \forall \perp$, then $B *_{c} A H \perp$

(*c -uniformity 1) given two consistent bases $A$ and $C$, if it holds for all $B^{\prime} \subseteq B$ that $B^{\prime} \cup\{\alpha\} \vdash \perp$ for every $\alpha \in A$ iff $B^{\prime} \cup\{\psi\} \vdash \perp$ for every $\psi \in C$, then $B \backslash\left(B *_{c} A\right)=B \backslash\left(B *_{c} C\right)$

( ${ }^{*}$-uniformity 2) given two consistent bases $A$ and $C$, if it holds for all $B^{\prime} \subseteq B$ that $B^{\prime} \cup\{\alpha\} \forall \perp$ for some $\alpha \in A$ iff $B^{\prime} \cup\{\psi\} \forall \perp$ for some $\psi \in C$, then $B \cap\left(B *_{c} A\right)=B \cap\left(B *_{c} C\right)$

$\left(*_{c}\right.$-relevance) if $\varphi \in B \backslash B *_{c} A$, then there is some $B^{\prime}$ with $B \cap\left(B *_{c} A\right) \subseteq B^{\prime} \subseteq B$, such that $B^{\prime} \cup\{\psi\} \forall \perp$ for some $\psi \in A$ and $B^{\prime} \cup\{\varphi\} \cup\{\lambda\} \vdash \perp$ for every $\lambda \in A$

( ${ }_{c}$-core retainment) if $\varphi \in B \backslash B{ }^{*}{ }_{c} A$, then there is some $B^{\prime} \subseteq B$, such that $B^{\prime} \cup\{\psi\} \forall \perp$ for some $\psi \in A$ and $B^{\prime} \cup\{\varphi\} \cup\{\lambda\} \vdash \perp$ for every $\lambda \in A$

$\left(*_{c}\right.$-iteration) $B *_{c} A=\left(B \cap\left(B *_{c} A\right)\right) *_{c} A$

(* ${ }_{c}$-coincidence) if $A \cap B \neq \varnothing$ and $A \subseteq C \subseteq(A \cup B)$, then $B{ }^{*} c A=B{ }_{c} C$

$\left(*_{c}\right.$-confirmation $)$ if $\left(A \cap\left(B *_{c} A\right)\right) \subseteq B$, then $B *_{c} A=B$

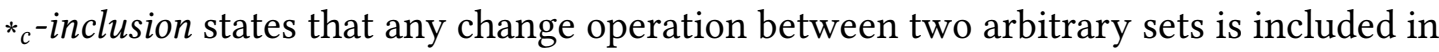
the union of them. ${ }^{*}{ }_{c}$-weak success ensures that if the input is non-empty and consistent, then a subset of it will be included in the final result. ${ }^{*}{ }_{c}$-vacuity 1 brings that nothing is changed if the input set is inconsistent. ${ }_{c}$-vacuity 2 establishes that if there is an intersection between the input and the original base, then nothing needs to be done. ${ }^{*_{c}}$-vacuity 3 determines that if the input set is consistent with the previous belief base, then the final result is equal to the union of them. ${ }^{*} c$-vacuity 4 brings the fact that if $B$ is consistent with the input set, then nothing is removed from $B .{ }^{*}{ }_{c}$-strong consistency guarantees that the final result is always consistent. ${ }^{*}{ }_{c}$-uniformity 1 states that if every piece of two consistent sets is inconsistent with the same subsets of the original belief base $B$, then the respective removed sentences of $B$ should be identical. ${ }_{c}^{*}$-uniformity 2 states that if both $A$ and $C$ have pieces consistent with the same subsets of the original belief base $B$, then the retained sentences of $B$ should be identical in both operations. ${ }_{c}{ }^{-}$-relevance and $*_{c}$-core-retainment bring the intuition that nothing is cut out from the original belief base except if its removal somehow contributes to making the new belief base consistent. ${ }^{*}{ }_{c}$-iteration establishes that if the preserved sentences of a base $B$ in a choice revision by $A$ are submitted to a new choice revision by $A$, then the result is the same. ${ }^{*}$-coincidence determines that if there is a non-empty intersection between $B$ and $A$ and $A$ is a subset of another base $C$ contained in the union of the $B$ and $A$, then the choice revision of $B$ by $A$ or $C$ gives the same outcome. ${ }^{*}{ }$-confirmation expresses the intuition that if the part of $A$ considered plausible and expected to be accepted is already included in the original belief base, then nothing needs to be done.

Among the listed postulates, ${ }^{*}$-vacuity 1 is an adaptation of a similar postulates from [FKIRS12]; ${ }^{*}$-vacuity 3 comes from [FKIRS12]; ${ }_{c}$-success, ${ }_{c}{ }_{c}$-relevance, ${ }_{c}$-iteration, ${ }^{*_{c}}$-coincidence and $*_{c}$-confirmation come from [Zha18]; ${ }^{*}$-uniformity 1 and $*_{c}$-uniformity 2 are adaptations from [FKIRS12] and [Zha18], and ${ }^{*}$-inclusion, ${ }^{*}{ }_{c}$-strong consistency and $*_{c}$-core retainment are adaptations of similar postulates from singleton revision [Han99b]. 
The remaining postulates $\left({ }^{*_{c}}\right.$-weak success, ${ }_{{ }_{c}}$-vacuity 2 and ${ }_{{ }_{c}}$-vacuity 4$)$ are new. ${ }_{c}$-weak success is necessary to represent the specific success condition of the operation. ${ }^{{ }_{c}}{ }^{*}$ vacuity 2 and ${ }^{*}$-vacuity 4 serve to express more possibilities for the operation's behaviour.

The observation below brings some relations among postulates:

Observation 17. (a) If an operator satisfies relevance, then it satisfies core-retainment.

(b) An operator satisfies uniformity 1 iff it satisfies uniformity 2.

(c) If an operator satisfies core-retainment, then it satisfies vacuity 4.

(d) If an operator satisfies vacuity 1, vacuity 2 and weak success, then it satisfies confirmation.

(e) If an operator satisfies vacuity 3 , then it satisfies vacuity 4 .

Although $*_{c}$-vacuity 4 follows from $*_{c}$-vacuity 3 , as seen above, ${ }^{*}{ }_{c}$-vacuity 4 is not superfluous because each of these postulates serves a different purpose. ${ }^{*}$-vacuity 3 represents the approach when in a situation of consistency between base and input you want everything in the final result (see Section 7.3). For the same premise, ${ }^{*}{ }_{c}$-vacuity 4 only guarantees that nothing is removed from the base, i.e. not necessarily the input set will be fully absorbed.

\subsection{Constructions}

By generalizing two specific approaches from classical base revision, it is possible to achieve two suitable constructions for choice revision: one based on remainder sets and the other on kernel sets.

\subsubsection{Partial Meet}

The Partial Meet construction is based on the concept of remainder set. Each element of the remainder set related to the choice revision of $B$ by $A$ is a maximal subset of $B$ consistent with some part of $A$. By maximal we mean that the addition of any other element of $B$ to an element of the remainder set would make the latter inconsistent with every element of $A$. Formally, it is possible to define a remainder set for choice revision that does not depend on the negation of formulas $\left(B \downarrow_{c} A\right)$ in the following way:

Definition 93. $X \in B \downarrow_{c} A, A \neq \varnothing$ iff:

- $X \subseteq B, X \neq \varnothing$;

- $\exists \psi \in A$ s.t. $X \cup\{\psi\} \forall \perp$;

- $\forall X^{\prime}$ s.t. $X \subset X^{\prime} \subseteq B$ it holds that $\forall \lambda \in A, X^{\prime} \cup\{\lambda\} \vdash \perp$.

From this definition, it is easy to see that if $B$ is consistent with some element of $A$ or if $B$ is consistent and there is a non-empty intersection between $B$ and $A$, then the remainder set is a unit set (with exactly $B$ ):

Observation 18. If there exists $\psi \in A$ such that $B \cup\{\psi\} \forall \perp$ or if $B \forall \perp$ and $B \cap A \neq \varnothing$, then $B \downarrow_{c} A=\{B\}$. 
In addition, if two sets $A$ and $C$ have consistent elements with exactly the same subsets of a belief base $B$, then their remainders coincide:

Observation 19. Let $A, B$ and $C$ be belief bases. If it holds for all $B^{\prime} \subseteq B$ that $B^{\prime} \cup\{\alpha\}$ is consistent for some $\alpha \in A$ iff $B^{\prime} \cup\{\psi\}$ is consistent for some $\psi \in C$, then $B \downarrow_{c} A=B \downarrow_{c} C$.

After computing the remainder set, a selection function will be responsible for choosing some element(s) of it:

Definition 94. [FKIRS12] Let $B$ be a belief base. $\gamma$ is a selection function for $B$ iff for every set $A$ :

1. If $B \downarrow_{c} A \neq \varnothing$, then $\varnothing \neq \gamma\left(B \downarrow_{c} A\right) \subseteq B \downarrow_{c} A$.

2. If $B \downarrow_{c} A=\varnothing$, then $\gamma\left(B \downarrow_{c} A\right)=\{B\}$.

It is interesting to observe that if $B$ and $A$ have sentences in common, these sentences will be part of every element of the remainder set:

Observation 20. Let $\gamma$ be a selection function for $B$. If $B \forall \perp$, then $B \cap A \subseteq \bigcap \gamma\left(B \downarrow_{c} A\right)$ for every $A$.

Applying the concepts of remainder set and selection function, the choice partial meet revision operator can be defined in the following way:

Definition 95. Let $B$ be a belief base, $\gamma$ a selection function for $B$ and $\dot{+}_{\perp}$ a consistencypreserving weak partial expansion operator for $B$. The multiple choice partial meet base revision on $B$ that is generated by $\gamma$ and $\dot{+}_{\perp}$ is the operator ${ }^{{ }_{\gamma+}}$ such that, for every set $A$, if $B \forall \perp$ or $B \cap A=\varnothing$ :

$$
B *_{\gamma+} A= \begin{cases}\bigcap_{B} \gamma\left(B \downarrow_{c} A\right) \dot{+}_{\perp} A & \text { if } A \neq \varnothing \text { and } A \forall \perp \\ \text { otherwise }\end{cases}
$$

An operator * is a multiple choice partial meet base revision on B iff there is a selection function $\gamma$ for $B$ and a consistency-preserving partial expansion operator $\dot{+}_{\perp}$ such that for all sets $B$, $B * A=B{ }_{\gamma+} A$.

Looking at the defined construction, it is possible to see that what remains from $B$ in the final result is exactly the intersection of what was chosen by the selection function:

Observation 21. Let ${ }_{\gamma_{+}}$be a multiple choice partial meet base revision as proposed in Definition 95. Then, if $B H \perp$ or $B \cap A=\varnothing, B \cap\left(B *_{\gamma_{+}} A\right)=\bigcap \gamma\left(B \downarrow_{c} A\right)$.

The next result states some properties satisfied by the operator defined above:

Observation 22. Let ${ }_{\gamma+}$ be a multiple choice partial meet base revision as proposed in Definition 95. Then ${ }^{*}{ }_{\gamma+}$ satisfies ${ }^{*}{ }_{c}$-iteration and ${ }_{c}$-coincidence.

The following representation theorem shows the equivalence between the defined operator and a set of postulates:

Theorem 66. An operator * is a multiple choice partial meet base revision for $B$ iff it satisfies: ${ }^{*}{ }_{c}$-inclusion, ${ }_{{ }_{c}}$-weak consistency, ${ }_{{ }_{c}}$-weak success, ${ }_{c}{ }_{c}$-vacuity $1, *_{c}$-uniformity 2 and 
$7.2 \mid$ CONSTRUCTIONS

$*_{c}$-relevance.

\subsubsection{Kernel}

The Kernel construction is based on the concept of kernel set. Each element of the kernel set related to the choice revision of $B$ by $A$ is a minimal subset of $B$ inconsistent with every part of $A$. By minimal we mean that any proper subset of $X$ is consistent with some part of $A$. Formally, it is possible to define a kernel set for choice revision that does not depend on the negation of formulas $\left(B \downarrow_{c} A\right)$ in the following way:

Definition 96. $X \in B \Downarrow_{c} A, A \neq \varnothing$ iff:

- $X \subseteq B, X \neq \varnothing$;

- $\forall \lambda \in A, X \cup\{\lambda\} \vdash \perp$;

- $\forall X^{\prime} \subset X \subseteq B, \exists \psi \in A$ s.t. $X^{\prime} \cup\{\psi\} \forall \perp$.

By this definition, if $B$ is consistent with a subset of $A$, then the kernel set will be empty:

Observation 23. If there exists $\psi \in A$ such that $B \cup\{\psi\} \forall \perp$ or if $B \forall \perp$ and $B \cap A \neq \varnothing$, then $B \Downarrow_{c} A=\varnothing$.

In addition, if $B$ is consistent and the pre-condition for $*_{c}$-coincidence is satisfied, then the kernel sets for $A$ and $C$ coincide:

Observation 24. Let $B$ be a consistent set. If $A \cap B \neq \varnothing$ and $A \subseteq C \subseteq(A \cup B)$, then $B \downarrow_{c} A=B \downarrow_{c} C$.

The next counterexample shows why $B$ needs to be consistent:

Example 4. Let $B=\{p, p \rightarrow q, s, \neg q\}, C=\{s, \neg q\}$ and $A=\{\neg q\}$. Hence, $A \cap B \neq \varnothing$ and $A \subseteq C \subseteq(A \cup B)$. We have that $B \downarrow_{c} A=\{\{p, p \rightarrow q\}\}$ but $B \downarrow_{c} C=\{\{p, p \rightarrow q, \neg q\}\}$.

After the definition of kernel set, we need an incision function that will be responsible for eliminating at least one sentence from each of the elements of the computed set:

Definition 97. [FKIRS12] Let $B$ be a belief base. $\sigma$ is an incision function for B iff, for all consistent set $A$ :

1. $\sigma\left(B \downarrow_{c} A\right) \subseteq \bigcup B \downarrow_{c} A$

2. If $X \in B \Downarrow_{c} A$, then $X \cap\left(\sigma\left(B \downarrow_{c} A\right)\right) \neq \varnothing$

From what has been defined so far, it is possible to infer that, if $B$ is consistent, the sentences in common between it and the input will not be part of any element of the kernel set:

Observation 25. Let $\sigma$ be an incision function for $B$. If $B \Vdash \perp$, then $B \cap A \subseteq\left(B \backslash \sigma\left(B \downarrow_{c} A\right)\right)$ for every $A$.

The example below, expressed in propositional logic, shows that Observations 23 and 25 may be not valid when $B$ is inconsistent: 
Example 5. Let $B=\{\alpha, \alpha \rightarrow \beta, \neg \beta\}$ and $A=\{\alpha, \beta\}$. Then $B \downarrow_{c} A=\{\{\alpha, \alpha \rightarrow \beta, \neg \beta\}\}$, which implies that, in this case, the kernel set is not empty and $B \cap A=\{\alpha\}$ may be chosen by the incision function.

Applying the concepts of kernel set and incision function, the choice kernel revision operator can be defined in the following way:

Definition 98. Let $B$ be a belief base, $\sigma$ be an incision function for $B$ and $\dot{+}_{\perp}$ a consistencypreserving weak partial expansion operator. The multiple choice kernel base revision on $B$ generated by $\sigma$ and $\dot{+}_{\perp}$ is the operator $*_{\sigma+}$ s.t., for every set $A$, if $B \forall \perp$ or $B \cap A=\varnothing$ :

$$
B *_{\sigma+} A= \begin{cases}\left(B \backslash \sigma\left(B \Downarrow_{c} A\right)\right) \dot{+}_{\perp} A & \text { if } A \neq \varnothing \text { and } A H \perp \\ B & \text { otherwise }\end{cases}
$$

An operator * is a choice kernel revision for $B$ iff there is an incision function $\sigma$ for $B$ and a consistency-preserving weak partial expansion operator $\dot{+}_{\perp}$ such that, for every set $A$, if $B \forall \perp$ or $B \cap A=\varnothing, B * A=B *_{\sigma+} A$.

The observation below brings an important intuition about the operator: what is preserved from $B$ after the revision process is exactly what remained after the incision:

Observation 26. Let $*_{\sigma+}$ be a multiple choice kernel base revision as proposed in Definition 98. Then, if $B \forall \perp$ or $B \cap A=\varnothing, B \cap\left(B *_{\sigma+} A\right)=B \backslash \sigma\left(B \Downarrow_{c} A\right)$.

Moreover, the next counterexample proves the necessity of the restrictions on $B$ and $A$ for the result:

Example 6. For a situation where $B$ is inconsistent and has a non-empty intersection with $A$, let $B=\{\alpha, \alpha \rightarrow \beta \wedge \delta, \neg \beta, \neg \delta\}$ and $A=\{\alpha, \varphi\}$. Then, the kernel set of $B$ by $A$ has two elements $X_{1}$ and $X_{2}: X_{1}=\{\alpha, \alpha \rightarrow \beta \wedge \delta, \neg \beta\}$ and $X_{2}=\{\alpha, \alpha \rightarrow \beta \wedge \delta, \neg \delta\}$.

Suppose that $\sigma\left(X_{1}\right)=\{\alpha\}$ and $\sigma\left(X_{2}\right)=\{\alpha \rightarrow \beta \wedge \delta\}$. Hence, $B *_{\sigma+} A=\left(B \backslash \sigma\left(B \downarrow_{c}\right.\right.$ $A)) \dot{+}_{\perp} A=\{\neg \beta, \neg \delta\} \dot{+}_{\perp} A$. A possible final result is $\{\neg \beta, \neg \delta, \alpha\}$. But, in this case, $B \cap\left(B *_{\sigma+}\right.$ $A)=\{\neg \beta, \neg \delta, \alpha\}$ and $B \backslash \sigma\left(B \Downarrow_{c} A\right)=\{\neg \beta, \neg \delta\}$, which proves that they are not equal.

The problem here is that a sentence from the intersection entered as part of an element of the kernel set, was chosen by the incision function and was later re-added by the partial expansion operator.

The next result states some properties satisfied by the defined construction and the example that follows after illustrates the need for the restrictions:

Observation 27. Let ${ }_{\sigma+}$ be a multiple choice kernel base revision as proposed in Definition 98. Then, if $B \forall \perp$ or $B \cap A=\varnothing$, ${ }_{\sigma+}$ satisfies ${ }^{*}{ }_{c}$-iteration and ${ }^{*}{ }_{c}$-coincidence.

Example 7. (Examples 4 and 6 revisited) Considering the same scenario as in Example 6, we have that $B *_{\sigma+} A=\{\neg \beta, \neg \delta\} \dot{+}_{\perp} A$ and a possible result for this revision is $\{\neg \beta, \neg \delta, \alpha\}$.

In order to analyze $*_{c}$-iteration, we have that $B \cap\left(B *_{\sigma+} A\right)=\{\neg \beta, \neg \delta, \alpha\}$. Thus, $\left(B \cap\left(B *_{\sigma+}\right.\right.$ A)) $\downarrow_{c} A=\varnothing\left(\right.$ Observation 23). Hence, $\left(B \cap\left(B *_{\sigma+} A\right)\right) *_{\sigma+} A=\{\neg \beta, \neg \delta, \alpha\} \dot{+}_{\perp} A$ and nothing guarantees that the weak partial expansion operator will give the same outcome from $B *_{\sigma+} A$. 
Regarding $*_{c}$-coincidence, from Example 4 we have that, since $B \cap A \neq \varnothing$, if $B$ is inconsistent, then $B \downarrow_{c} A$ may be different from $B \Downarrow_{c} C$, which implies that the result $B *_{\sigma+} A=B *_{\sigma+} C$ is not guaranteed.

Now we have the kernel version of Observation 19: if every element of two sets $A$ and $C$ are inconsistent with exactly the same subsets of a belief base $B$, then their kernel sets coincide:

Observation 28. Let $A, B$ and $C$ be belief bases. If it holds for all $B^{\prime} \subseteq B$ that $B^{\prime} \cup\{\alpha\} \vdash \perp$ for every $\alpha \in A$ iff $B^{\prime} \cup\{\psi\} \vdash \perp$ for every $\psi \in C$, then $B \downarrow_{c} A=B \downarrow_{c} C$.

In order to show the equivalence between the defined operator and a set of postulates, we obtained the following representation theorem:

Theorem 67. An operator * is a multiple choice kernel base revision for B iff it satisfies: ${ }^{*}{ }_{c}$-inclusion, ${ }^{*}$-weak consistency, ${ }^{*}{ }_{c}$-weak success, ${ }^{*}{ }_{c}$-vacuity $1,{ }^{*}{ }_{c}$-uniformity 1 and $*_{c}$-core retainment.

From representation theorems 66 and 67 and Observation 17 it is possible to infer that the multiple partial meet choice revision operators satisfy all the postulates that axiomatically characterize the multiple kernel choice revision operators. The corollary below expresses this result:

Corollary 2. Let $B$ be a belief base. If $*_{c}$ is a multiple choice partial meet base revision for $B$, then $*_{c}$ is a multiple choice kernel base revision for $B$.

\subsection{Maximal Expansion}

An interesting case deserves our attention: when the input $A$ is consistent with the base $B$. Although their simple union of them results in a consistent set, the product of a kernel choice revision of $B$ by $A$ depends on the partial expansion operator. It is possible to establish a specific kind of partial expansion operator which works as a classical expansion operator when $B$ is consistent with $A$, i.e., $B+A=B \cup A$. Based on this scenario, it is possible to derive a new postulate for partial expansions:

(+-maximal expansion) if $B \cup A \forall \perp$, then $B \cup A \subseteq B+A$

The theorem below is a variant of Theorem 35 that covers this new possibility:

Theorem 68. Let + be a partial expansion operator that satisfies the postulates described in Theorem $35 .+$ is a maximal partial expansion iff it in addition satisfies + -maximal expansion.

Finally, the following observation shows the consequence of this variant on the properties of a choice revision operator based on it:

Observation 29. Let $B$ be a belief base, + be a partial expansion operator and $*_{c}$ a multiple kernel (partial meet) choice revision for $B$ based on $\dot{+}$. If $\dot{+}$ is a maximal partial expansion, then ${ }^{*}$ satisfies ${ }^{*}$-vacuity 3 . 


\subsection{Minimal Work}

There is another possibility for choice revision that consists in doing nothing when there is a non-empty intersection between the belief base and the input set. The idea, in this case, is that the success condition was previously satisfied (given that there is at least one element of the input in the original base) and nothing else needs to be done.

For this purpose, the constructions could be adapted in the following way:

Definition 99. Let $B$ be a belief base, $\gamma(\sigma)$ a selection (incision) function for $B$ and $\dot{+}_{\perp} a$ consistency-preserving weak partial expansion operator for $B$. The multiple choice partial meet base revision (multiple choice kernel base revision) on $B$ that is generated by $\gamma(\sigma)$ and $\dot{+}_{\perp}$ is the operator ${ }^{*}{ }_{\gamma+}\left({ }^{*}{ }_{\sigma+}\right)$ such that, for every set $A$ :

$$
\begin{gathered}
B *_{\gamma_{+}} A= \begin{cases}\bigcap_{B} \gamma\left(B \downarrow_{c} A\right) \dot{+}_{\perp} A & \text { if } A \neq \varnothing, A \forall \perp \text { and } B \cap A=\varnothing \\
\text { otherwise }\end{cases} \\
B *_{\sigma+} A= \begin{cases}\left(B \backslash \sigma\left(B \downarrow_{c} A\right)\right) \dot{+}_{\perp} A & \text { if } A \neq \varnothing, A \forall \perp \text { and } B \cap A=\varnothing \\
B & \text { otherwise }\end{cases}
\end{gathered}
$$

An operator * is a multiple choice partial meet base revision (multiple choice kernel base revision) on $B$ iff there is a selection (incision) function $\gamma(\sigma)$ for $B$ and a consistency-preserving partial expansion operator $\dot{+}_{\perp}$ such that, for every set $A, B * A=B *_{\gamma_{+}} A\left(B * A=B *_{\sigma+} A\right)$.

The following observation shows the consequence of this modified operator on a property of choice revisions obtained from it:

Observation 30. Let $B$ be a belief base, + be a partial expansion operator and $*_{c}$ a multiple choice partial meet (kernel) base revision for B based on + as proposed in Definition 99 . Then ${ }^{*}$ satisfies ${ }{ }_{c}$-vacuity 2.

\subsection{Example}

For illustration purposes, we can imagine the following context. Suppose that we believe that birds fly, that birds are cold-blooded and that Tweety is a bird. Naturally, every flying animal and every cold-blooded animal is an animal. Then we receive a bunch of new beliefs telling us that Tweety does not fly and that birds are neither animals nor cold-blooded. This situation could be expressed using a Description Logic syntax:

Initial beliefs (B): (I) Bird $\sqsubseteq$ FlyingAnimal, (II) FlyingAnimal $\sqsubseteq$ Animal, (III) Bird(Tweety), (IV) Bird ᄃ ColdAnimal, (V) ColdAnimal $\sqsubseteq$ Animal.

New beliefs (A): Bird $\sqsubseteq \neg$ Animal, $\neg$ FlyingAnimal(Tweety), Bird $\sqsubseteq \neg$ ColdAnimal.

Now it is possible to calculate both the remainder set and the kernel set.

$B \downarrow_{c} A=\{\{I, I I, I I I, V\},\{I, I I, I V, V\},\{I, I I I, I V\},\{I I, I I I, I V, V\}\}$ 


$$
B \downarrow_{c} A=\{\{I, I I, I I I, I V\},\{I, I I I, I V, V\}\}
$$

For partial meet revision, it is necessary to choose some element(s) of the remainder to perform their intersection and then decide what will be absorbed from the input. Suppose that the selection function chooses the first and the fourth elements. The intersection would result in $\{$ Flying Animal $\sqsubseteq$ Animal, Bird(Tweety), ColdAnimal $\sqsubseteq$ Animal $\}$. It permits the whole input set to be incorporated without generating inconsistency. However, it does not mean that it would be the best option, since Bird $\sqsubseteq \neg$ Animal does not seem a reasonable information to believe.

For kernel revision, it is necessary to perform an incision in each of the elements of the kernel set. Suppose that the incision function chooses only the belief IV (which would be possible since it appears in both elements). The modified base would be $\{$ Bird $ᄃ$ Flying Animal, FlyingAnimal $\sqsubset$ Animal, ColdAnimal $\sqsubseteq$ Animal, Bird(Tweety)\}. From the input set, the only belief that could not be absorbed is the first one.

\subsection{Connections between Selective Revision and Choice Revision}

When comparing the general idea of choice revision with the multiple operator for belief bases defined in the preceding chapter, it is possible to notice some similarities. More specifically, a more direct comparison is possible looking at a multiple selective base revision operator that satisfies choice success (since the choice revision operators defined in this chapter do not work with logical consequences of the input, but just with explicit beliefs). Thus, if you look only at the final result of both operations (selective and choice), the outcome represents the same mechanism: a belief base revised to accommodate a subset of the input.

A natural question that arises is: are these operations equivalent? If yes, under what conditions?

\subsubsection{From Selective to Choice}

The first analysis is whether a selective operator is a choice revision. In order to be a multiple choice partial meet base revision, a selective operator has to satisfy ${ }^{*_{c}}$-inclusion, ${ }^{*}{ }_{c}$-weak consistency, ${ }^{*}$-weak success, ${ }^{*}$-vacuity $1,{ }^{*}$-uniformity 2 and ${ }_{c}$-relevance. Since our comparison is with the selective operator with choice success, according to Theorem 64 the set of properties satisfied by the defined multiple selective base revision operator is inclusion, weak consistency, conditional uniformity, choice success, conditional success, uniform success and weak relevance.

More directly, ${ }^{*}$-inclusion, ${ }^{*}$-weak consistency and ${ }^{*}{ }_{c}$-weak success are satisfied. For ${ }^{*}{ }_{c}$-vacuity, we have two cases. If $A=\varnothing$, then $f(A)=\varnothing$ (according to $f$-choice) but $B{ }^{*} p \varnothing=B$ only if $B$ is consistent. If $A \neq \varnothing$, even when $A \vdash \perp$, then $A \cap B \odot A \neq \varnothing$ (because of choice success) and nothing guarantees that $B \odot A=B$. Thus, there should be an equivalent property for selective. 
For ${ }^{*}$-relevance, if $A \subseteq B \odot A$, then it is readily satisfied. Otherwise, it depends on the multiple package revision again $\left(B \odot A=B{ }^{*} f(A)\right)$. Since ${ }^{*} p$ satisfies relevance, we have that if $\varphi \in B \backslash B{ }^{*} f(A)$, then there exists $B^{\prime}$ such that $B \cap\left(B *_{p} f(A)\right) \subseteq B^{\prime} \subseteq B$, $B^{\prime} \cup f(A) \forall \perp$ but $B^{\prime} \cup\{\varphi\} \cup f(A) \vdash \perp$. Looking at ${ }_{{ }_{c}}$-relevance, given that $B^{\prime} \cup f(A) \forall \perp$ we have that $B^{\prime} \cup\{\psi\} \forall \perp$ for some $\psi \in A$. However, $B^{\prime} \cup\{\varphi\} \cup f(A) \vdash \perp$ does not imply that $B^{\prime} \cup\{\varphi\} \cup\{\lambda\} \vdash \perp$ for every $\lambda \in A$. There should be a specific property for this situation.

Regarding $*_{c}$-uniformity 2 , we are going to consider $*_{c}$-uniformity 1 instead (Observation 17 , item $\mathrm{b}$ ). Assume that given two consistent bases $A$ and $C$ it holds for all $B^{\prime} \subseteq B$ that $B^{\prime} \cup\{\alpha\} \vdash \perp$ for every $\alpha \in A$ iff $B^{\prime} \cup\{\psi\} \vdash \perp$ for every $\psi \in C$. Then it also holds that $B^{\prime} \cup A \vdash \perp$ iff $B^{\prime} \cup C \vdash \perp$. If $A \subseteq B \odot A$ we are done. Otherwise, it depends on ${ }_{p}$, since $B \odot A=B{ }_{p} f(A), f(A) \subseteq A$. However, although ${ }_{p}$ satisfies uniformity (Theorem 15), even when $B^{\prime} \cup A \vdash \perp$ iff $B^{\prime} \cup C \vdash \perp$ nothing guarantees that $B^{\prime} \cup f(A) \vdash \perp$ iff $B^{\prime} \cup f(C) \vdash \perp$. There should be also a specific property for $f$ in this case.

Observation 31. A multiple selective base revision operator is a multiple choice partial meet base revision iff it satisfies in addition:

(vacuity 2) if $A=\varnothing$ or $A \vdash \perp$, then $B \odot A=B$

(choice relevance) if $\varphi \in B \backslash B \odot A$, then there exists $B^{\prime}$ such that $B \cap(B \odot A) \subseteq B^{\prime} \subseteq B$, $B^{\prime} \cup \psi \forall \perp$ for some $\psi \in A$ but $B^{\prime} \cup\{\varphi\} \cup \lambda \vdash \perp$ for every $\lambda \in A$.

(f-uniformity) if it holds for all $B^{\prime} \cup B$ that $B^{\prime} \cup A \vdash \perp$ iff $B^{\prime} \cup C \vdash \perp$, then $B^{\prime} \cup f(A) \vdash \perp$ iff $B^{\prime} \cup f(C) \vdash \perp$

\subsubsection{From Choice to Selective}

Now we do the converse analysis. In order to be a multiple selective base revision, a choice revision operator has to satisfy inclusion, weak consistency, conditional uniformity, choice success, conditional success, uniform success and weak relevance. According to Theorem 66, the set of properties satisfied by a multiple choice partial meet operator is $*_{c}$-inclusion, ${ }^{*}$-weak consistency, ${ }^{*}$-weak success, ${ }^{*}$-vacuity $1,{ }_{c}{ }_{c}$-uniformity 2 and $*_{c}$ relevance.

More directly, inclusion and weak consistency are satisfied. For conditional success we have that if $A \backslash B \subseteq B{ }^{*} A$, then $A \subseteq B{ }^{*}{ }_{c} A$, since from Observation 20 we know that the intersection $A \cap B$ is preserved by the operation. For choice success, we have from ${ }^{*}{ }_{c}$ weak success that, if $A \neq \varnothing$ and $A \forall \perp$, then there is a set $C \subseteq A$ such that $C \subseteq B{ }^{*}$ A. However, nothing guarantees that $B *_{c} A=B *_{c} C$; an extra restriction would be necessary. For uniform success, there is no correspondent condition in $*_{c}$.

Regarding weak relevance and weak uniformity, following the same motivation from selective, there should be a property that guarantees that if $A \subseteq B{ }^{*} A$, then $*_{c}$ works as a package revision.

Observation 32. A multiple choice partial meet base revision is a multiple selective base revision iff it satisfies in addition:

(iteration 2) $B *_{c} A=B *_{c}\left(A \cap\left(B *_{c} A\right)\right)$ 
(success) $A \cap\left(B *_{c} A\right) \neq \varnothing$

(uniform success) If $A \subseteq B *_{c} A$ and for all subsets $B^{\prime} \subseteq B, B^{\prime} \cup A \vdash \perp$ iff $B^{\prime} \cup C \vdash \perp$, then $A \subseteq B{ }^{*} A$ iff $C \subseteq B *_{c} C$

(package uniformity) If $A \subseteq B{ }^{*} A$ and for all $B^{\prime} \subseteq B, B^{\prime} \cup A \vdash \perp$ iff $B^{\prime} \cup C \vdash \perp$, then $B \cap(B * A)=B \cap(B * C)$

(package relevance) If $A \subseteq B *_{c} A$ and there is some $\beta \in B$ such that $\beta \notin B *_{c} A$, then there is some $B^{\prime}$ such that $B{ }_{c} A \subseteq B^{\prime} \subseteq B \cup\{A\}, B^{\prime}$ is consistent but $B^{\prime} \cup\{\alpha\}$ is inconsistent

\subsection{Related Work}

The first definition of Multiple Choice Revision was given in [Fuh88]. Given two sets $K$ and $A$, Fuhrmann states that whatever the sentences from $A$ that an agent decides to add to $K$, there will be needed an incision on $K$. So, according to the thesis, the agent should "retract only those sentences which are, by comparison, the least important (the most retractable) ones". Representing this idea using the Levi Identity, we have: $K{ }^{*} A=$ $\left[K-_{c} \neg A\right]+A \cap\left\{\alpha: \neg \alpha \notin\left[K-_{c} \neg A\right]\right\}^{1}$, where $\left[K-_{c} \neg A\right]$ represents the choice contraction of $K$ by those elements of $\neg A$ that are "easiest to retract". Besides the fact that this definition was given for propositional logic (including negation of sentences) and belief sets, there was no axiomatic characterization (nor postulates neither representation theorems).

Zhang [Zha18] proposed two types of choice revision: one based on the Levi Identity (contraction + expansion) and the other on external revision (expansion + contraction). Both of them were axiomatically characterized. Although these approaches were developed for bases, they depend on negation and disjunction of sentences and on multiple contraction operations. Our approach can be applied to logics without negation and proposes a more direct construction for revision (without using contraction as an intermediate step). In addition, while Zhang's approach was proposed only for consistent initial belief bases, our operators restrict on consistency only if there is an intersection between the initial base and the input set. As a final consideration, our proposal covers some variants of the problem that Zhang does not (as shown in Section 7.3).

In [Han17], Hansson introduced a proposal to choice revision based on another approach to belief change named Descriptor Revision. This approach applies a "select-direct" procedure by considering that there is a set of belief sets that work as possible results of belief change and this change is implemented through a direct choice among these possible results. This proposal for choice was further developed by Zhang [Zha19] and the described operator was axiomatically characterized. Differently from the operator in [Zha18] and more similar to ours, the Descriptor Revision-based operator was defined without using contraction as an intermediate step. On the other hand, it was proposed for belief sets instead of belief bases, which differs from our work. Furthermore, our approach is not based on a "select-direct" methodology for belief change.

\footnotetext{
${ }^{1}$ In his thesis, $\neg A$ is defined as the set $\{\neg \alpha: \alpha \in A\}$.
} 



\section{Chapter 8}

\section{Algorithms}

Among the advantages of using belief bases is obtaining a more suitable model for computational purposes. In addition, it is desirable to have a paved path for future applications of the Belief Revision theory explored here.

After defining constructions and proving their relation with a list of postulates, it is possible to derive algorithms that show how to compute the remainder or kernel set for the constructions. In this chapter, we present some algorithms for Multiple Revision, both for package and choice, together with demonstrations of their correctness. The algorithms were based on [Rib10, Res14].

All the auxiliary algorithms that compute just an element of the remainder or kernel set are black-box type, in the sense that they call a reasoner we know nothing about.

\subsection{Multiple Package Revision}

As seen in Chapter 3, Falappa et. al. [FKIRS12] proposed two constructions for package revision, focusing on belief bases and without using contraction as an intermediate step. In this section we are going to propose algorithms that aim to obtain the kernel and remainder sets related to that approach.

\subsubsection{Package Partial Meet Revision}

The first algorithm is an auxiliary function that receives a belief base $B$, an input set $A$ and a subset $X$ of $B$ (which may be empty) and computes an element of the remainder set of $B$ by $A$ of which $X$ is a subset. This computation is done by iterating over the whole $B$ and trying to check, for each element, if after its addition to $X$ the resulting set is consistent with $A$. If yes, the corresponding element of $B$ is added.

Note that every $X$ received is a potential seed for an element of the remainder, which justifies the restriction on $X$ being consistent with $A$. This condition is essential to achieve an element of the remainder. 


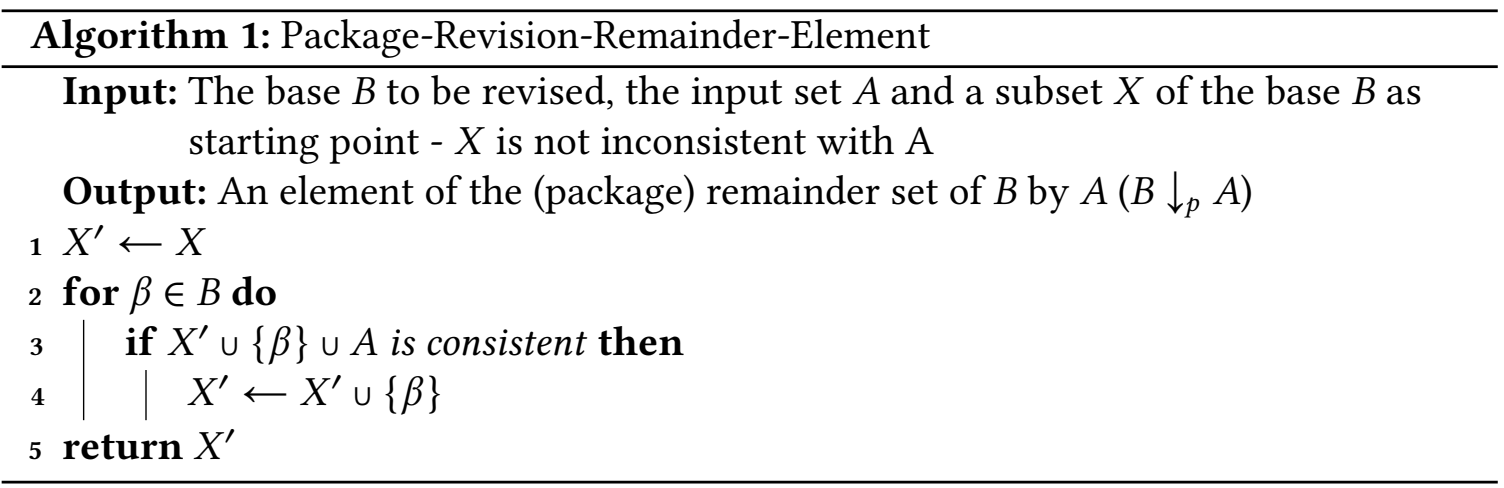

Proposition 11. Algorithm Package-Revision-Remainder-Element returns an element of $B \downarrow_{p} A$.

Proof. We need to prove that $X^{\prime}$ returned by the algorithm is an element of the remainder set of the package revision of $B$ by $A$ (considering that the $X$ given as argument is not inconsistent with $A$ ).

Suppose, towards contradiction, that the algorithm returns an $X^{\prime}$ such that $X^{\prime} \notin B \downarrow_{p} A$. Then, either $X^{\prime}$ is inconsistent with $A$ or there exists $Y$ such that $X^{\prime} \subset Y \subseteq B$ and $Y$ is consistent with $A$ (that is, $X^{\prime}$ is not maximal).

Suppose that the algorithm returned $X^{\prime}$ such that $X^{\prime}$ is inconsistent with $A$. In the algorithm, the sentences of $B$ are added to $X^{\prime}$ one by one (loop between lines 2 and 4 ) and, every time one of them will make $X^{\prime}$ inconsistent with $A$, it is not added to the set. This way, $X^{\prime}$ is progressively composed on line 4 of the algorithm and only if the new sentence to be added, altogether with the sentences possibly already present in $X^{\prime}$, does not make $X^{\prime}$ inconsistent with $A$ (condition checked on line 3). So, there is an invariant on line 2: $X^{\prime}$ is consistent with $A$. Contradiction.

Suppose that there exists $Y$ such that $X^{\prime} \subset Y \subseteq B$ and $Y$ is consistent with $A$. Then there is some $\beta \in Y$ such that $\beta \notin X^{\prime}$. If $\beta$ was not added to $X^{\prime}$, it made the condition on line 3 fail. Thus, $X^{\prime} \cup\{\beta\}$ is inconsistent with $A$. Contradiction, because $\left\{X^{\prime} \cup\{\beta\}\right\} \subseteq Y$ and $Y$ is consistent with $A$.

Therefore, Package-Revision-Remainder-Element returns an element of $B \downarrow_{p} A$.

The next algorithm (Package-Revision-Remainder-Set) receives a belief base $B$ and an input set $A$ and calculates the whole remainder set for package revision. It starts with the computation of one element of the remainder and, if $B \cup A$ is inconsistent, then for every sentence of the complement of $B$ in relation to the computed element the algorithm inserts it on a queue, which will be explored until it becomes empty. The justification for this procedure is that any other element of the remainder must have at least one sentence that is not part of the returned element. The queue becomes, then, the next potential elements of the remainder set.

For each element $\mathrm{Hn}$ of the queue, if it is really a potential element of the remainder (by checking its consistency with $A$ ), then Package-Revision-Remainder-Element is called again, giving $H n$ as $X$. If the returned subset $S$ was never reached before, it is stored 
and, for every sentence of the complement of $B$ in relation to $S$, the union of it and $H n$ is inserted on the queue.

Hence, the idea of the algorithm is to start with one element of the remainder set and explore it in order to reach the others.

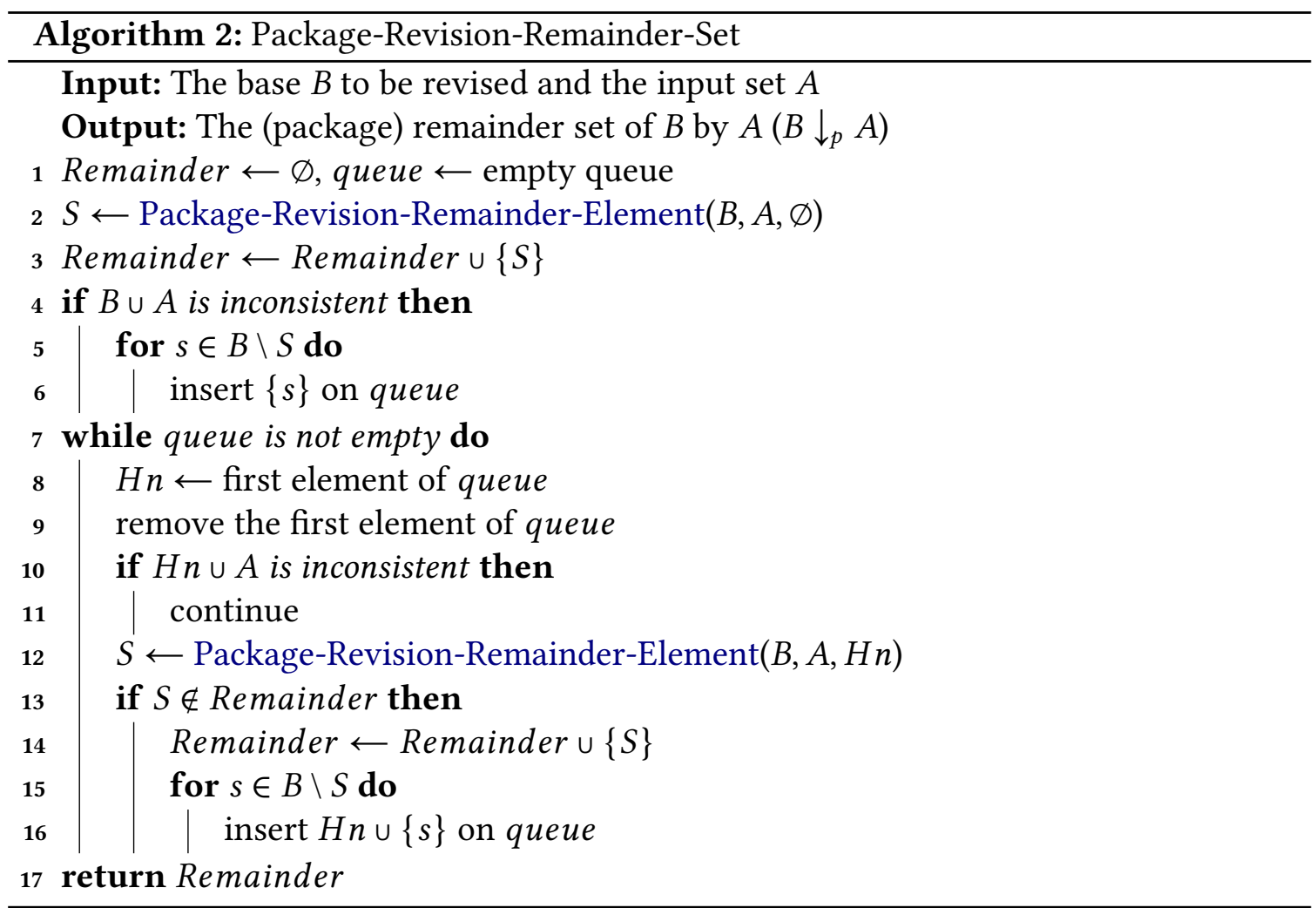

Proposition 12. Algorithm Package-Revision-Remainder-Set returns the remainder set of the package revision of $B$ by $A\left(B \downarrow_{p} A\right)$.

Proof. In relation to this algorithm that aims to find all the elements of $B \downarrow_{p} A$, we need a two-step proof:

1 - To prove that $S$ on line 12 is an element of $B \downarrow_{p} A$.

2 - To prove that, if $X \in B \downarrow_{p} A$ and is not computed as $S$ on line 2, then it will be computed as $S$ on line 12 .

Part 1: The first part is simpler because, as $H n$ is not inconsistent with $A$, it is a direct consequence of the fact that Package-Revision-Remainder-Element returns en element of $B \downarrow_{p} A$.

Suppose towards contradiction that there is some $S$ obtained on line 12 such that $S \notin B \downarrow_{p} A$. Then either $S \cup A$ is inconsistent or there exists $S^{\prime}$ such that $S \subset S^{\prime} \subseteq B$ and $S^{\prime}$ is not inconsistent with $\mathrm{A}$ ( $S$ is not maximal).

Suppose that $S$ is inconsistent with $A$. Then, either $H n$ is inconsistent with $A$ (which is not possible due to the condition on line 10) or Package-Revision-Remainder-Element 
returned an $S$ such that $S$ is inconsistent with $A$, which is not possible according to Proposition 11. Contradiction.

Now, suppose that there exists $S^{\prime}$ such that $S \subset S^{\prime} \subseteq B$ and $S^{\prime}$ is consistent with $A$. Then Package-Revision-Remainder-Element returned an $S$ that is not maximal (and, consequently, not an element of $B \downarrow_{p} A$ ). Contradiction (Proposition 11).

Therefore, $S$ on line 12 is an element of $B \downarrow_{p} A$.

Part 2: In order to prove this part, we are going to consider the invariant of the queue valid on line 7 of the loop between lines 7-16. We have that for all $X \in B \downarrow_{p} A$ either $X \in$ Remainder or there exists $A \in$ queue such that $A \subseteq X$. Now, we are going to show that this invariant is valid.

Let $X$ be an element of $B \downarrow_{p} A$. If $X$ is not obtained on line 2 as the first element of $B \downarrow_{p} A$ returned by Package-Revision-Remainder-Element, then there exists some $a \in B \backslash S$ such that $a \in X$ and, thus, $\{a\}$ will be added to the queue (lines 5-6). The invariant is valid.

Now let us consider the loop between lines 7-16. Hn receives each element of the queue. Every time $H n \subset X$ there is a possibility for Package-Revision-Remainder-Element to return $X$ (line 12), as the $H n$ from the corresponding iteration is a subset of the set $S$ obtained on line 12 in that same iteration. Let us consider the case when it happens $(H n \subseteq X)$. If Package-Revision-Remainder-Element returns $X$, so this $A$-remainder is found and stored as a remainder. Otherwise, there exists some $a \in B \backslash S$ such that $a \in X$, and $H n \cup\{a\}$ is added to the queue (lines 15-16). Then, the invariant is still valid.

Since the queue is empty on line 17 , we have that Remainder $=B \downarrow_{p} A$. Therefore, if $X \in B \downarrow_{p} A$ and is not computed as $S$ on line 2, then $X$ will be computed as $S$ on line 12 .

\subsubsection{Package Kernel Revision}

Differently from the one for remainder sets, the kernel version of black-box algorithm receives just the belief base $B$ and the input $A$ and, in order to find one element of the kernel set, it applies a strategy known as Expand-Shrink. First, during the expand part, an initially empty set is incremented with sentences from the base $B$, one by one, until it is inconsistent with $A$. Then the shrink part begins, checking every element of the newly built set in order to test if it is necessary to entail the inconsistency and, if not, the corresponding element is removed. It is this last part that guarantees minimality.

Proposition 13. Algorithm Package-Revision-Kernel-Element returns an element of $B \downarrow_{p} A$.

Proof. We need to show that $B^{\prime}$ returned by the algorithm is such that $B^{\prime} \in B \downarrow_{p} A$. Suppose towards contradiction that the algorithm returns a $B^{\prime}$ such that $B^{\prime} \notin B \downarrow_{p} A$. Then, either $B^{\prime} \cup A$ is consistent or there exists $X \subset B^{\prime} \subseteq B$ such that $X \cup A$ is inconsistent (it means, $B^{\prime}$ is not minimal).

Suppose that $B^{\prime} \cup A$ is consistent. As $B^{\prime}$ starts empty and is constructed on line 3 , the condition on line 4 altogether with the loop interruption command on line 5 gives us an invariant on line 2 (the beginning of the loop between lines 2 and 5): $B^{\prime} \cup A$ is consistent. 


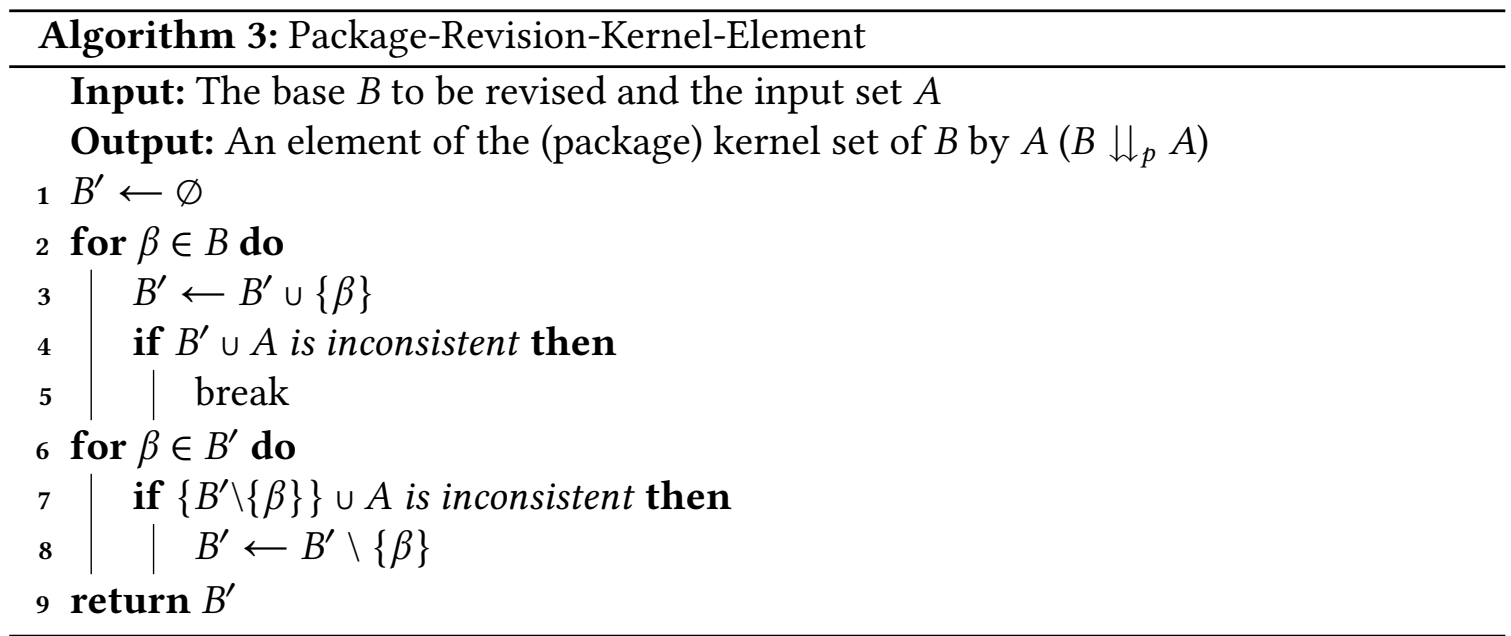

Thus, this loop is interrupted only when $B^{\prime} \cup A$ becomes inconsistent. On line 6 of the loop between lines 6 and 8 , there is an invariant: $B^{\prime} \cup A$ is inconsistent. In this loop, an element $\beta \in B^{\prime}$ is removed from $B^{\prime}$ only if $\left\{B^{\prime} \backslash\{\beta\}\right\} \cup A$ is inconsistent and the loop only terminates when $B^{\prime}$ is completely explored. Therefore, a $B^{\prime}$ returned by the algorithm is such that $B^{\prime} \cup A$ is inconsistent. Contradiction.

Suppose that there exists $X$ such that $X \subset B^{\prime} \subseteq B$ and $X \cup A$ is inconsistent. Then there is some $\beta \in B^{\prime}$ such that $\left\{B^{\prime} \backslash\{\beta\}\right\} \cup A$ is inconsistent. As the loop between lines 6 and 8 only terminates when $B^{\prime}$ is completely explored, $\beta$ is removed from $B^{\prime}$ due to the condition on line 7 . Therefore, $\beta \notin B^{\prime}$ returned by the algorithm. Contradiction. A.

Therefore, algorithm Package-Revision-Kernel-Element returns an element of $B \downarrow_{p}$

The next algorithm (Package-Revision-Kernel-Set) receives a belief base $B$ and an input set $A$ and calculates the whole kernel set for package revision. Similarly to the one for remainder, it starts with the computation of one element of the kernel but only if $B$ is inconsistent with $A$ (otherwise, the kernel should be empty). For every sentence of the computed element, the algorithm inserts it on a queue, which will be explored until it becomes empty.

For each element $H n$ of the queue, Package-Revision-Kernel-Element is called again but passing $B \backslash H n$ as the belief base to be considered. The justification for this procedure is that any other element of the kernel must be achieved from a belief base that differs with $B$ on at least one element. However, Package-Revision-Kernel-Element is called only if $B \backslash H n$ is inconsistent with $A$. If the returned subset $S$ was never reached before, it is stored and, for every sentence of $S$, the union of it and $H n$ is inserted on the queue.

Hence, similarly to the one for remainders, the idea of the algorithm is to start with one element of the kernel set and to explore it in order to reach the others.

Proposition 14. Algorithm Package-Revision-Kernel-Set returns the kernel set of $B$ in relation to the set $A$ by means of package revision ( $\left.B \downarrow_{p} A\right)$.

Proof. In relation to this main algorithm that intends to find all the elements of the kernel 


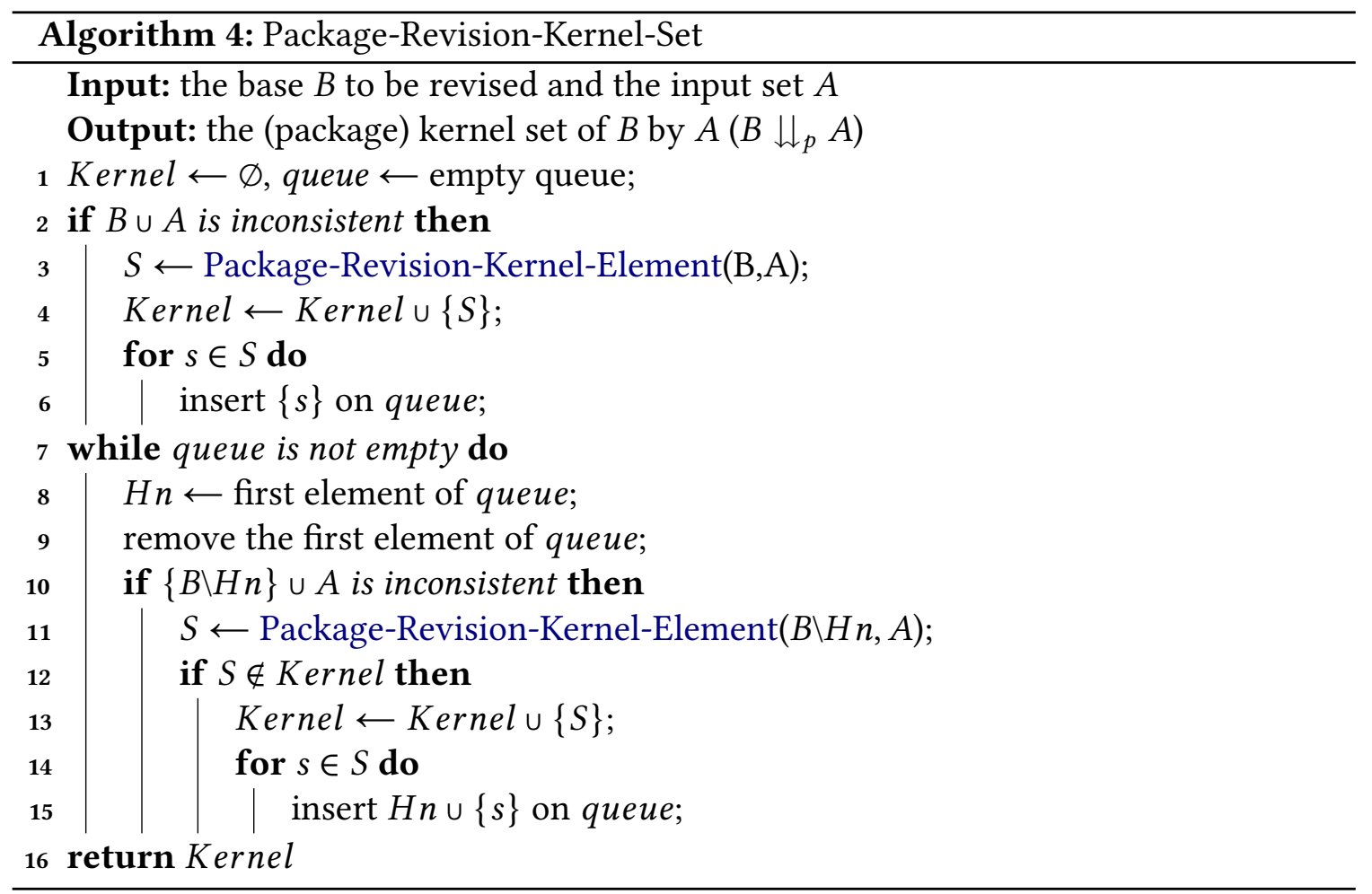

set of $B$ in relation to $A$ by means of package revision, we need a two-step proof: 1 - To prove that $S$ on line 11 is an element of $B \downarrow_{p} A$.

2 - To prove that if $X \in B \downarrow_{p} A$ then $X$ will be computed as $S$ on line 11 .

\section{Part 1:}

Since in algorithm Package-Revision-Kernel-Set line 11 is invoked only when $\{B \backslash$ $H n\} \cup A$ is inconsistent, we have that the proof of this first part is a direct consequence of the correctness of algorithm Package-Revision-Kernel-Element (Proposition 13).

\section{Part 2:}

To this part, we have to observe the invariant valid on line 7 of the loop between lines 7 and 15. We have that $\forall X \in B \downarrow_{p} A$, either $X \in$ Kernel or exists $A \in$ queue such that $X \subset B \backslash A$. Now we are going to show why it is valid.

Let $S$ be the set obtained on line 3 of the algorithm and $X$ be an element of $B \downarrow_{p} A$. If $X \neq S$, so there exists some $a \in S$ such that $a \notin X$ and $\{a\}$ will be put on the queue. The invariant is valid here.

Now consider the loop between lines 7 and 15, letting $S$ be from now on the set obtained on line 11. $H n$ receives each element of the queue. Everytime $H n \cap X=\varnothing$ there is the possibility of receiving $X$ from algorithm Package-Revision-Kernel-Element (line 11), since the set given as first argument is $B \backslash H n(B \backslash H n$ inconsistent with $A)$, it means, $H n \nsubseteq S$, where $S$ is the set obtained on line 11 of the iteration. If Package-Revision-Kernel-Element returns $X$, this element found is inserted in Kernel. Otherwise, there exists some $a \in S$ such that $a \notin X$ and $H n \cup\{a\}$ will be put on the queue (lines 14-15). The invariant is valid 
in this case as well.

Since on line 15 the queue is empty, we have that Kernel $=B \downarrow_{p} A$. Thus, if $X$ is an element of the kernel set and is not computed as $S$ on line 3, it will be computed as $S$ on line 11.

\subsection{Multiple Choice Revision}

In Chapter 7, we proposed two constructions for choice revision, focusing on belief bases and without using contraction as an intermediate step. In this section, we are going to propose algorithms that aim to obtain the kernel and remainder sets.

\subsubsection{Choice Partial Meet Revision}

The black-box algorithm for choice works in a very similar way to the one for package. The strategy for finding one element of the remainder set is almost the same, except for the fact that, instead of checking consistency with $A$, this time it checks consistency with every element of $A$ until it finds a positive answer. Once again, every $X$ received is a potential element of the remainder, but the restriction on it changes: it cannot be inconsistent with every element of $A$.

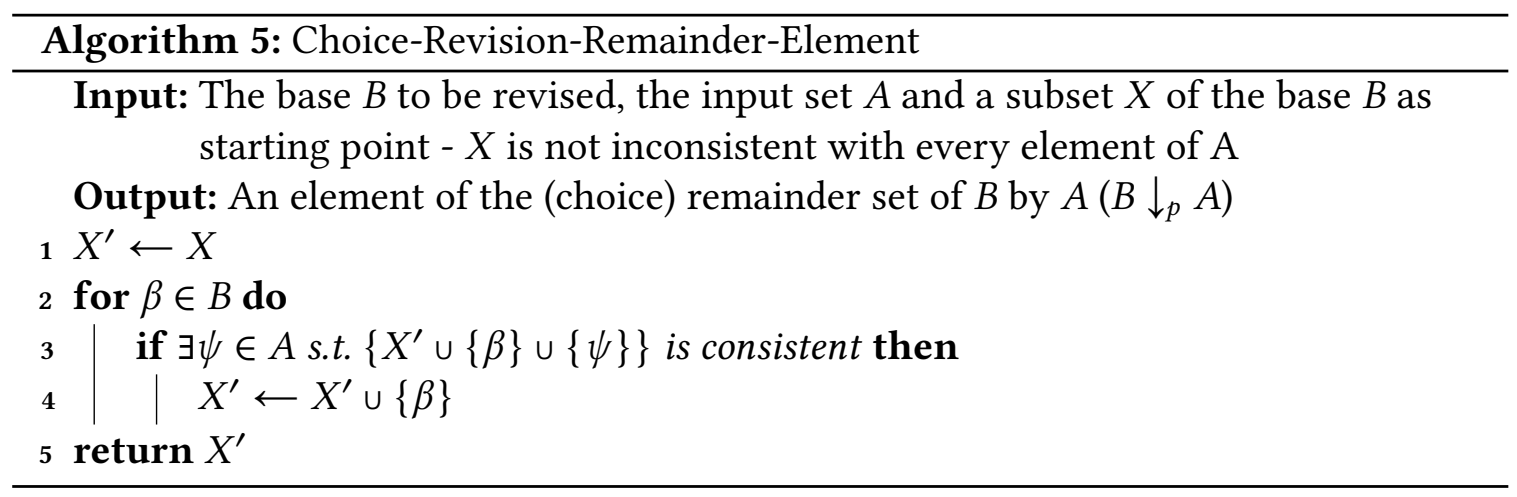

Proposition 15. Algorithm Choice-Revision-Remainder-Element returns an element of $B \downarrow_{c} A$.

Proof. We need to prove that the set $X^{\prime}$ returned by the algorithm is an element of the remainder set of a choice revision of $B$ by $A$ (considering that the $X$ passed as argument is not inconsistent with every element of $A$ ).

Suppose towards contradiction that the algorithm returns an $X^{\prime}$ such that $X^{\prime} \notin B \downarrow_{c} A$. Then, either $X^{\prime}$ is inconsistent with every $\lambda \in A$ or $\exists K, X^{\prime} \subset K \subseteq B$, and $\exists \psi \in A$ such that $K$ is consistent with $\{\psi\}$ (that is, $X^{\prime}$ is not maximal).

Suppose that the algorithm returned an $X^{\prime}$ that is inconsistent with every element of $A$. In the algorithm, the sentences of $B$ are added to $X^{\prime}$ one by one (loop between lines 2 and 4) and, every time one of them will make $X^{\prime}$ inconsistent with every element of $A$, it is not added to the set. This way, $X^{\prime}$ is progressively composed by line 4 of the algorithm and only if the new sentence to be added, altogether with the sentences possibly already 
present in $X^{\prime}$, does not make $X^{\prime}$ inconsistent with every element of $A$ (condition checked on line 3). So, there is an invariant on line 2: $\exists \psi \in A$ such that $X^{\prime} \cup\{\psi\}$ is consistent. Contradiction. Thus, $X^{\prime}$ returned by the algorithm is not inconsistent with every element of $A$.

Suppose that $\exists K, X^{\prime} \subset K \subseteq B$ and $\exists \psi \in A$ such that $K \cup\{\psi\}$ is consistent. Then $\exists \beta \in K$ such that $\beta \notin X^{\prime}$. Since $B$ is completely explored (line 2 ), if $\beta$ was not added to $X^{\prime}$, it made the condition on line 3 fail. Thus, $\forall \lambda \in A, X^{\prime} \cup\{\beta\}$ is inconsistent with $\{\lambda\}$. Contradiction. Thus, $\nexists K, X^{\prime} \subset K \subseteq B$, such that $\exists \psi \in A$ and $K \cup\{\psi\}$ is consistent.

Therefore, Choice-Revision-Remainder-Element returns an element of $B \downarrow_{c} A$.

The next algorithm (Choice-Revision-Remainder-Set) receives a belief base $B$ and an input set $A$ and calculates the whole remainder set for choice revision. The strategy is very similar to the one for package. In addition to the black-box algorithm called, the main difference in on the checking of $\mathrm{Hn}$ : now it is ignored if it is inconsistent with every element of $A$.

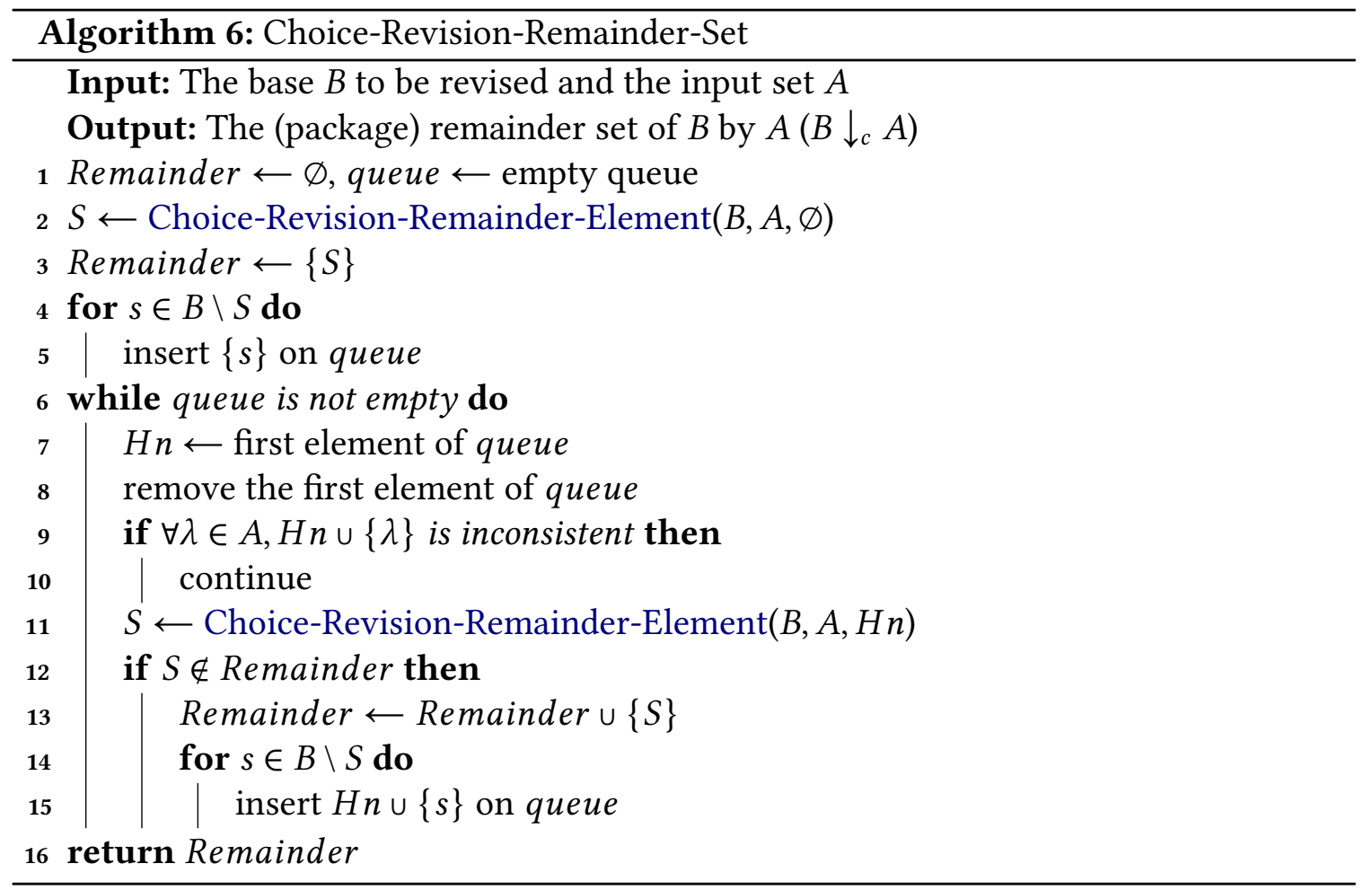

Proposition 16. Algorithm Choice-Revision-Remainder-Set returns $B \downarrow_{c} A$ (the remainder set of the choice revision of $B$ by $A$ ).

Proof. In relation to this algorithm that aims to find all the elements of $B \downarrow_{c} A$, we need a two-step proof:

1 - To prove that $S$ on line 11 is an element of $B \downarrow_{c} A$.

2 - To prove that, if $X \in B \downarrow_{c} A$ and is not returned on line 2, then it will be computed as $S$ on line 11. 
Part 1: The first part is simpler because, since the $H n$ used on line 11 is not inconsistent with every subset of $A$, the proof is a direct consequence of the fact that Choice-RevisionRemainder-Element returns an element of $B \downarrow_{c} A$ (Proposition 15).

Suppose towards contradiction that there is some $S$ obtained on line 11 such that $S \notin B \downarrow_{c} A$. Then either $\forall A^{\prime} \subseteq A, S \cup A^{\prime}$ is inconsistent or $\exists S^{\prime}$ such that $S \subset S^{\prime} \subseteq B$ and $S^{\prime}$ is not inconsistent with every subset of $A$ (that is, $S$ is not maximal).

Suppose that an $S$ is inconsistent with every subset of $A$. Then, either $H n$ was inconsistent with every subset of $A$ (which is not possible due to the condition on line 9) or Choice-Revision-Remainder-Element returned such $S$, which is not possible according to Proposition 15. Contradiction. Thus, $\exists A^{\prime} \subseteq A$ such that $S \cup A^{\prime}$ is consistent.

Now, suppose that $\exists S^{\prime}$ such that $S \subset S^{\prime} \subseteq B$ and $S^{\prime}$ is consistent with some subset of $A$. So Choice-Revision-Remainder-Element returned an $S$ that is not maximal (and, consequently, not an element of $B \downarrow_{c} A$ ). Contradiction (according to Proposition 15). Thus, $\nexists S^{\prime}$ such that $S \subset S^{\prime} \subseteq B$ and $S^{\prime}$ is consistent with some subset of $A$.

Therefore, every $S$ obtained on line 11 is an element of $B \downarrow_{c} A$.

Part 2: In order to prove this part, we are going to consider an invariant valid on line 6 of the loop between lines 6-15. We have that $\forall X \in B \downarrow_{c} A$ either $X \in$ Remainder or $\exists Q \in$ queue such that $Q \subseteq X$. Now, we are going to show that this invariant is valid.

Let $X$ be an arbitrary element of $B \downarrow_{c} A$. If $X$ is not obtained on line 2 as the first element of $B \downarrow_{c} A$ returned by Choice-Revision-Remainder-Element, then $\exists s \in B \backslash S$ such that $s \in X$ and, then, $\{s\}$ will be added to the queue (lines 4-5). The invariant is valid.

Now let us consider the loop between lines 6-15. Hn receives the elements of the queue. Every time $H n \subseteq X$ there is a possibility for Choice-Revision-Remainder-Element to return $X$ (line 11), since the $H n$ from the corresponding iteration is a subset of the set $S$ to be obtained on line 11 in that same iteration. Let us consider the case when it happens $(H n \subseteq X)$. If Choice-Revision-Remainder-Element returns $X$, so this $A$-remainder is found and stored as a remainder. Otherwise, $\exists s^{\prime} \in B \backslash S$ such that $s^{\prime} \in X$, and $H n \cup\left\{s^{\prime}\right\}$ is added to the queue (lines 14-15). Then, the invariant is still valid.

Since the queue is empty on line 16, we have that Remainder $=B \downarrow_{c} A$. Therefore, if $X \in B \downarrow_{c} A$, so $X$ will be computed as $S$ either on line 2 or on line 11 .

\subsubsection{Choice Kernel Revision}

Again, the choice version of the black-box algorithm for kernel is very similar to the one for package since it applies the Expand-Shrink strategy. The main point of difference is on the consistency check: now it checks consistency with every element of $A$.

Proposition 17. Algorithm Choice-Revision-Kernel-Element returns an element of $B \downarrow_{p} A$.

Proof. We need to show that the set $B^{\prime}$ returned by the algorithm is such that $B^{\prime} \in B \downarrow_{c} A$.

Suppose towards contradiction that the algorithm returns a $B^{\prime}$ such that $B^{\prime} \notin B \downarrow_{c} A$. Then, either $\exists \psi \in A$ such that $B^{\prime} \cup\{\psi\}$ is consistent or $\exists K \subset B^{\prime}$ such that $\forall \lambda \in A, K \cup\{\lambda\}$ 


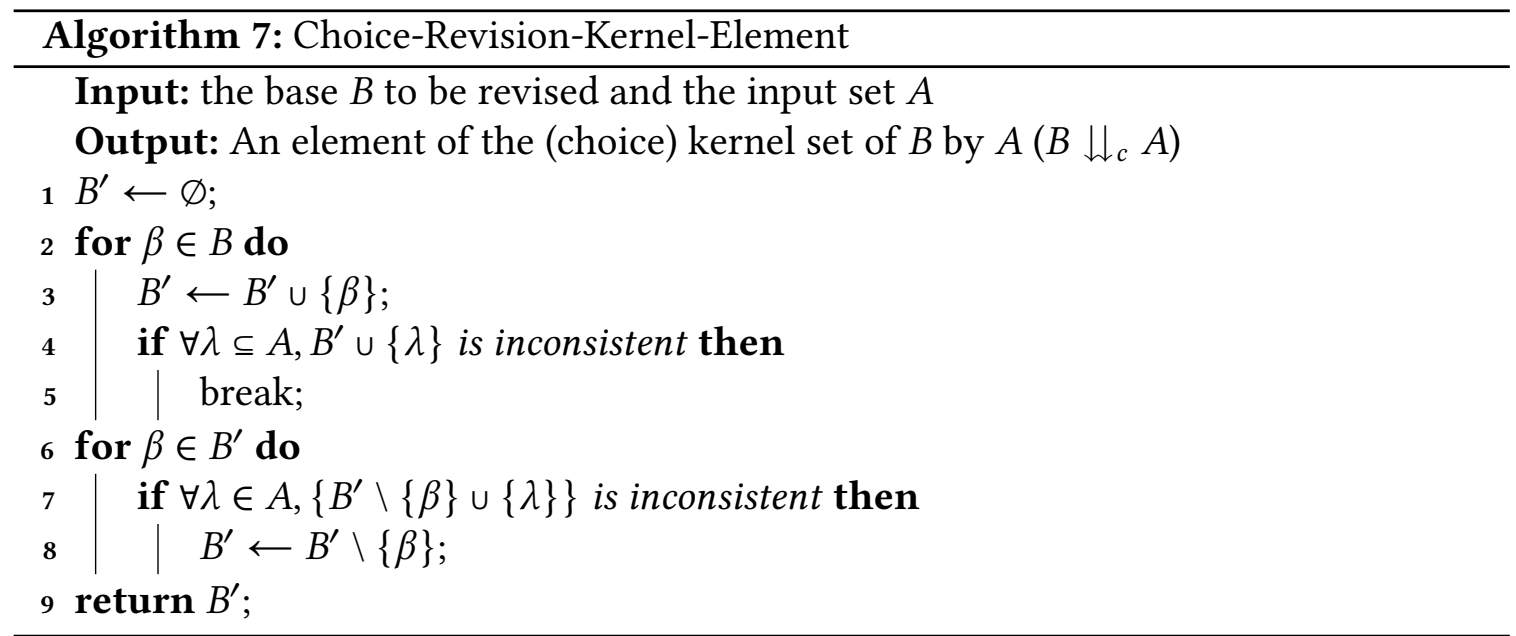

is inconsistent (it means, $B^{\prime}$ is not minimal).

Suppose that $\exists \psi \in A$ such that $B^{\prime} \cup\{\psi\}$ is consistent. As $B^{\prime}$ starts empty and is constructed on line 3 , the condition on line 4 altogether with the loop interruption command on line 5 gives us an invariant on line 2 (the beginning of the loop between lines 2 and 5): $\exists \psi \in A$ such that $B^{\prime} \cup\{\psi\}$ is consistent. Thus, this loop is interrupted only when $\forall \lambda \in A, B^{\prime} \cup\{\lambda\}$ is inconsistent. On line 6 of the loop between lines 6 and 8 , there is an invariant: $\forall \lambda \in A, B^{\prime} \cup\{\lambda\}$ is inconsistent. In this loop, an element $\beta \in B^{\prime}$ is removed from $B^{\prime}$ only if $\forall \lambda \in A,\left\{B^{\prime} \backslash\{\beta\}\right\} \cup\{\lambda\}$ is inconsistent and the loop only terminates when $B^{\prime}$ is completely explored. Therefore, a $B^{\prime}$ returned by the algorithm is such that $\forall \lambda \in A, B^{\prime} \cup\{\lambda\}$ is inconsistent. Contradiction. Thus, $\nexists \psi \in A$ such that $B^{\prime} \cup\{\psi\}$ is consistent.

Suppose that $\exists K \subset B^{\prime}$ such that $\forall \lambda \in A, K \cup\{\lambda\}$ is inconsistent. Then $\exists \beta \in B^{\prime}$ such that $\left\{B^{\prime} \backslash\{\beta\}\right\} \cup\{\lambda\}$ is inconsistent, $\forall \lambda \in A$. Since the loop between lines 6 and 8 only terminates when $B^{\prime}$ is completely explored, $\beta$ is removed from $B^{\prime}$ due to the condition on line 7. Therefore, $\beta \notin B^{\prime}$ returned on line 9. Contradiction. Thus, $B^{\prime}$ is minimal.

Therefore, Choice-Revision-Kernel-Element returns an element of $B \downarrow_{c} A$.

Finally, the next algorithm (Choice-Revision-Kernel-Set) receives a belief base $B$ and an input set $A$ and calculates the whole kernel set for choice revision. The strategy is very similar to the one for package: it starts with the computation of one element of the kernel, but only if there is no element of $A$ consistent with $B$ (see Observation 23). Later, the main difference is on the consistency check for $B \backslash H n$ : it validates consistency with every element of $A$.

Proposition 18. Algorithm Choice-Revision-Kernel-Set returns $B \downarrow_{c} A$ (the kernel set of the choice revision of $B$ by $A$ ).

Proof. In relation to this main algorithm that intends to find all the elements of the kernel set of $B$ in relation to $A$ by means of choice revision, we need a two-step proof:

1 - To prove that $S$ on line 11 is an element of $B \downarrow_{c} A$.

2 - To prove that if $X \in B \downarrow_{c} A$ and is not computed on line 3 then $X$ will be computed as 


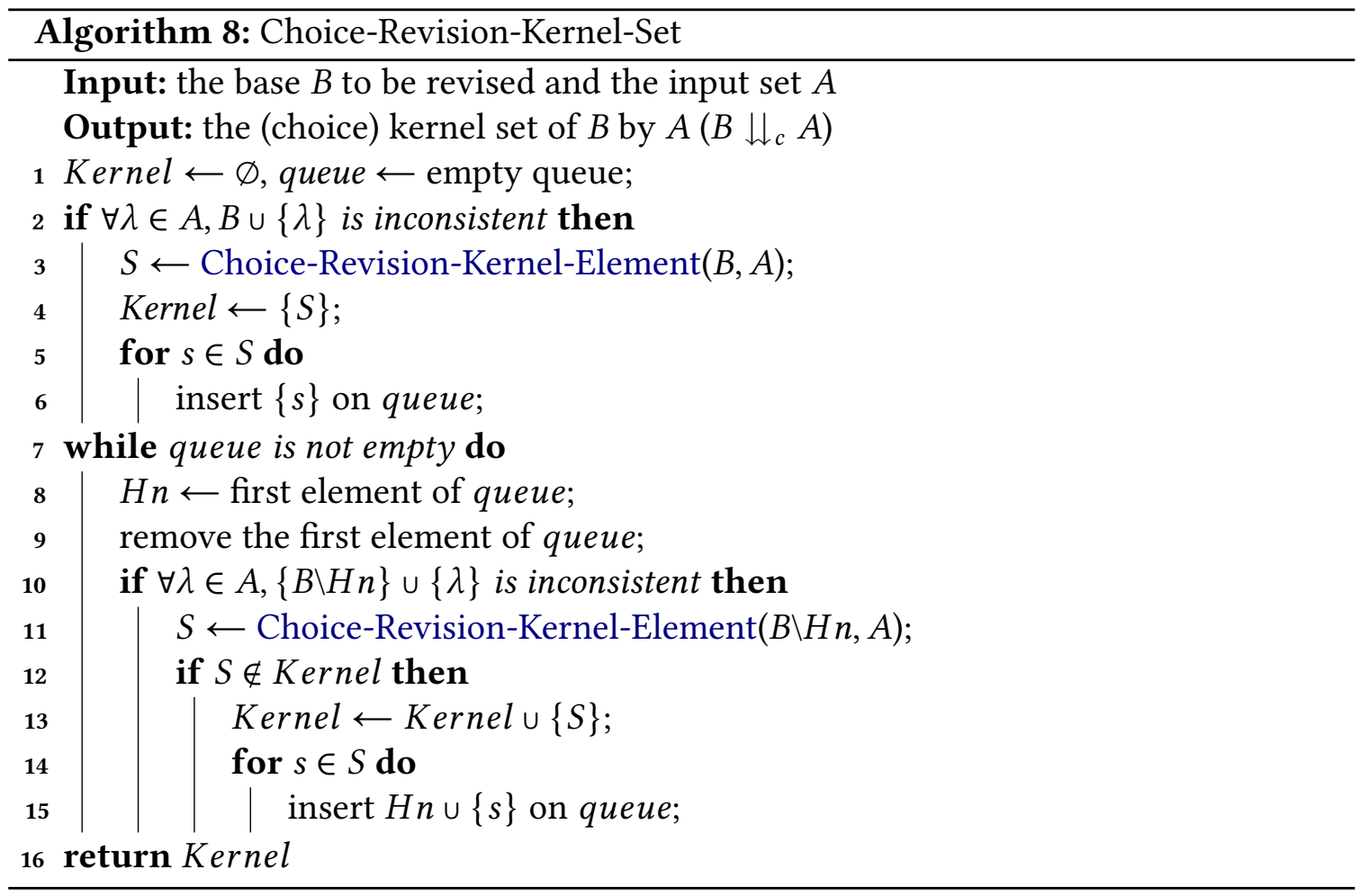

$S$ on line 11.

Part 1:

Since on line 11 the algorithm is invoked only when $\forall \lambda \in A,\{B \backslash H n\} \cup\{\lambda\}$ is inconsistent, we have that the proof of this first part is a direct consequence of the correctness of ChoiceRevision-Kernel-Element (Proposition 17).

Part 2:

To this part, we have to observe the invariant valid on line 7 of the loop between lines 7 and 15. We have that $\forall X \in B \downarrow_{c} A$, either $X \in$ Kernel or $\exists Q \in$ queue such that $X \subset B \backslash Q$. Now we are going to show why it is valid.

Let $S$ be the set obtained on line 3 of the algorithm and $X$ be an element of $B \downarrow_{c} A$. If $X \neq S$, then $\exists s \in S$ such that $s \notin X$ and $\{s\}$ will be put on the queue. The invariant is valid here.

Now consider the loop between lines 7 and 15, letting $S$ be from now on the set obtained on line 11. $H n$ receives the elements of the queue. Every time $H n \cap X=\varnothing$ there is the possibility of receiving $X$ from Choice-Revision-Kernel-Element (line 11), since the set given as first argument is $B \backslash H n$ (which is inconsistent with any element of $\mathrm{A}$ ), it means, $H n \nsubseteq S$, where $S$ is the set to be obtained on line 11 of the iteration. If Choice-RevisionKernel-Element returns $S=X$, this element found is inserted in Kernel. Otherwise, $\exists s \in S$ such that $s \notin X$ and $H n \cup\{s\}$ will be put on the queue (lines 14-15). The invariant is valid in this case as well. 
Since on line 16 the queue is empty, we have that Kernel $=B \downarrow_{c} A$. Thus, if $X$ is an element of the kernel set and is not computed as $S$ on line 3, it will be computed as $S$ on line 11 . 


\section{Chapter 9}

\section{Conclusion}

In this thesis, we presented a literature overview covering several models of Multiple Belief Revision, both prioritized and non-prioritized. We did not include the works on Iterated Revision since our focus was on models in which the beliefs of the incoming set are processed simultaneously. One of our aims was to unify the terminology and the symbols used in the area. When applicable, we observed the possibility or not of reduction to singleton revision.

After the survey on existing work, we focused on selective revision - a revision operation that may reject part of the input. We adapted the selective revision operators that were defined in [FH99] to the belief base context. We proposed new properties for the transformation functions, several new postulates to characterize selective base revision operators and presented axiomatic characterizations for several classes of such operators. We also addressed the issue of revision in languages which are not closed under negation, as is the case of Description Logics and Horn logic. Instead of defining revisions based on contraction through the Levi Identity, we adapted negation free revision to the context of selective revision.

In addition, we showed a detailed original study of Multiple Selective Revision. Based on existing definitions and characterizations of both multiple and singleton selective revision, we studied the generalization of partial acceptance via selective revision to make it possible to deal with sets of sentences as input. Multiple Selective Revision can be constructed by means of applying a transformation function to the input and then performing a prioritized multiple revision by the transformed input. We have provided lists of plausible postulates for the operation and also for the transformation functions, and relations between them were observed. Constructions were defined and representation theorems showed the connection between the postulates and the constructions. In the three different classes defined for multiple selective revision, the transformation function could choose a subset of the input set, a logical weakening of a chosen subset or even elements not related to the input. All this generalization was proposed for both beliefs sets and belief bases. In the particular case where $f(A)=A$ holds for all $A$, multiple selective revision coincides with multiple package revision.

Finally, we worked on the generalization of belief base revision to the multiple choice 
kind. We axiomatically characterized different classes of choice revision operators: some based on remainder sets (Partial Meet) and others on kernel sets. We analyzed the relations among several postulates for choice and defined an additional scenario to the Partial Expansion operation. It is also important to note that our results do not depend on negation of formulas and, hence, can be applied to a wider range of logics (and not only to classical propositional logic).

In the end, we proposed algorithms to find the remainder and kernel sets, both in package and choice contexts. They were designed in a general way in the sense of not depending on a specific logic or on a particular reasoner. They were also proved to be correct.

\subsection{Future Work}

Concerning Multiple Selective Revision, more properties of the operators could be explored, such as the generalized AGM supplementary postulates and their relation with the selective context. Besides, Kernel could be studied as another possible construction, along with an adapted version of core-retainment. In addition, more scenarios could be analyzed, for example, when the input is inconsistent but has a consistent subset. Still, a research opportunity is to define negation free selective revision for belief sets and generalize it to the multiple case.

Regarding Multiple Choice Revision, future work also includes the study of how to deal with inconsistent inputs that have a consistent subset. Other option is to explore a scenario when the agent wants to (consistently) incorporate from the input as much as possible. It is different from what was proposed in Section 7.3, where we considered the case when the input set is consistent with the belief base and the agent is able to incorporate everything; the point here is when the input is inconsistent with the base and the agent wants not only any subset of the input but as much as possible. Another research opportunity is to work with credibility values associated to the beliefs of the input or associated to the beliefs' sources, and these values would be used to guide the selection or the incision function. It is also open the study and definitions of potential relations between Multiple Choice Revision and Merge: although some aspects of the operations are similar (since they are of non-prioritized kind), they are not directly interchangeable because in Merge the operation is of symmetric change.

Another possible future work is to study how to generalize Belief Update [KM91a] to the multiple case and, more specifically, regarding the non-prioritized aspects studied in this thesis. 


\section{Appendix A}

\section{Proofs related to Chapters 5 and \\ 6}

\section{Proof of Observation 12}

Proof. $\quad$ 1. Let $\alpha \in B$. It holds that $f$ satisfies lower boundary. Thus $f(\alpha)=\alpha$. Thus $B \circledast \alpha=B * f(\alpha)=B * \alpha$. By *-success it follows that $\alpha \in B * \alpha$. Thus $\alpha \in B \circledast \alpha$.

2. Let $\alpha \in B \circledast \alpha$. Hence $\alpha \in B * f(\alpha)$. From which it follows, by *-inclusion, that $\alpha \in B \cup\{f(\alpha)\}$. Thus either $\alpha \in B$ or $\alpha=f(\alpha)$. It holds that $f$ satisfies lower boundary, thus in both cases it holds that $\alpha=f(\alpha)$. Hence $B \circledast \alpha=B * f(\alpha)=B * \alpha$. From which it follows by *-inclusion that $B \circledast \alpha \subseteq B \cup\{\alpha\}$.

3. Let $\{\alpha\} \forall \perp$. Hence $\forall \neg \alpha$. It holds that $f$ satisfies consistency preservation. Thus $\forall \neg f(\alpha)$. Hence $\{f(\alpha)\} \forall \perp$. Therefore, by *-consistency, it follows that $B * f(\alpha) \forall \perp$, from which it follows by definition of $\circledast$ that $B \circledast \alpha H \perp$.

4. It holds that $\forall \neg f(\alpha)$. Hence $\{f(\alpha)\} H \perp$, from which it follows, by *-consistency, that $B * f(\alpha) \forall \perp$. Thus $B \circledast \alpha \forall \perp$.

5. It holds that $B \circledast \alpha=B * f(\alpha)$. By *-success it follows that $f(\alpha) \in B * f(\alpha)=B \circledast \alpha$. On the other hand, $f$ satisfies idempotence, thus $f(f(\alpha))=f(\alpha)$. Therefore, $B$ * $f(\alpha)=B * f(f(\alpha))$. By definition of $\circledast$ it follows that $B * f(f(\alpha))=B \circledast f(\alpha)$. Hence $f(\alpha) \in B \circledast \alpha=B \circledast f(\alpha)$.

6. As shown in the previous item, it holds that $f(\alpha) \in B \circledast \alpha=B \circledast f(\alpha)$. On the other hand, $f$ satisfies implication, thus $\vdash \alpha \rightarrow f(\alpha)$.

7. Let $\alpha \in B \circledast \alpha$. Hence $\alpha \in B * f(\alpha)$. From which it follows, by *-inclusion, that $\alpha \in B \cup\{f(\alpha)\}$. Thus either $\alpha \in B$ or $\alpha=f(\alpha)$. It holds that $f$ satisfies lower boundary, thus in both cases it holds that $\alpha=f(\alpha)$. Hence $B \circledast \alpha=B * \alpha$. Let $\beta \in B$ and $\beta \notin B \circledast \alpha$. Thus, by *-relevance, it follows that there is some $B^{\prime}$ such that $B \oplus \alpha \subseteq B^{\prime} \subseteq B \cup\{\alpha\}, B^{\prime} H \perp$ but $B^{\prime} \cup\{\beta\} \vdash \perp$.

8. The proof that $\circledast$ satisfies weak core-retainment is similar to the proof presented for weak relevance. 
9. Consider that it holds for all subsets $B^{\prime}$ of $B$ that $B^{\prime} \cup\{\alpha\} \vdash \perp$ iff $B^{\prime} \cup\{\beta\} \vdash \perp$. Hence $f(\alpha)=\alpha$ holds iff $f(\beta)=\beta$ holds (since $f$ satisfies uniform identity). Assume that $\alpha \in B \circledast \alpha$. Hence $\alpha \in B * f(\alpha)$. From which it follows by $*$-inclusion that $\alpha \in B$ or $f(\alpha)=\alpha$. In both cases it follows that $f(\alpha)=\alpha$ (since $f$ satisfies lower boundary). On the other hand, $f$ satisfies uniform identity, thus $f(\beta)=\beta$. By definition of $\circledast$ it holds that $B \circledast \beta=B * f(\beta)$. By *-success it follows that $f(\beta) \in B \circledast \beta$. Thus $\beta \in B \circledast \beta$. By symmetry of the case it holds that if $\beta \in B \circledast \beta$, then $\alpha \in B \circledast \alpha$. Hence it holds that $\alpha \in B \circledast \alpha$ iff $\beta \in B \circledast \beta$.

10. Let $\alpha \in B \circledast \alpha$. Consider that it holds for all subsets $B^{\prime}$ of $B$ that $B^{\prime} \cup\{\alpha\} \vdash \perp$ iff $B^{\prime} \cup\{\beta\} \vdash \perp$. It holds that $\circledast$ satisfies uniform success (as shown in the previous item), thus $\beta \in B \circledast \beta$. On the other hand, from $\alpha \in B \circledast \alpha$ it follows that $f(\alpha)=\alpha$ (as shown above). By symmetry of the case it follows that $f(\beta)=\beta$. On the other hand, by *-uniformity, it follows that $B \cap B * \alpha=B \cap B * \beta$. Thus $B \cap B \circledast \alpha=B \cap B * f(\alpha)=$ $B \cap B * \alpha=B \cap B * \beta=B \cap B * f(\beta)=B \cap B \otimes \beta$.

\section{Proof of Theorem 51}

Proof. (a) implies (b):

We will first define $f$ and *.

Let $f: \mathcal{L} \times \mathcal{P}(\mathcal{L}) \longrightarrow \mathcal{L}$ be such that:

$$
f(\alpha)= \begin{cases}\alpha & \text { if } \alpha \in B \circledast \alpha \\ r(\alpha) & \text { otherwise }\end{cases}
$$

where $\mathrm{r}$ is a function $\mathcal{L} \times \mathcal{P}(\mathcal{L}) \longrightarrow \mathcal{L}$ such that $r(\alpha) \in B \circledast \alpha, B \circledast \alpha=B \circledast r(\alpha)$ and $\vdash \alpha \rightarrow r(\alpha)$.

Let

$$
B * \alpha= \begin{cases}B \circledast \alpha & \text { if } \alpha \in B \circledast \alpha \\ B \star^{\prime} \alpha & \text { otherwise }\end{cases}
$$

where $\star^{\prime}$ is a partial meet base revision operator.

We need to show that:

(a) $f$ is a (well-defined) transformation function;

(b) * satisfies inclusion, success, consistency, uniformity and relevance (by Theorem 1);

(c) $B \circledast \alpha=B * f(\alpha)$, for all $\alpha$.

Since $\star^{\prime}$ is a partial meet base revision, according to Theorem 1 it satisfies inclusion, success, consistency, uniformity and relevance.

(a) To prove that $f$ is a (well defined) function we must show that for all $\alpha \in \mathcal{L}$ there exists $\alpha^{\prime} \in \mathcal{L}$ such that $f(\alpha)=\alpha^{\prime}$ and that, if $\alpha_{1}=\alpha_{2}$, then $f\left(\alpha_{1}\right)=f\left(\alpha_{2}\right)$.

Let $\alpha \in \mathcal{L}$. If $\alpha \in B \circledast \alpha$, then $f(\alpha)=\alpha$. If $\alpha \notin B \circledast \alpha$, then $f(\alpha)=r(\alpha)=\alpha^{\prime}$, for 
some $\alpha^{\prime}$ such that $\alpha^{\prime} \in B \circledast \alpha=B \circledast \alpha^{\prime}$ and $\vdash \alpha \rightarrow \alpha^{\prime}$. Such $\alpha^{\prime}$ exists since $\circledast$ satisfies proxy success. Assume now that $\alpha_{1}=\alpha_{2}$. If $\alpha_{1} \in B \circledast \alpha_{1}$, then $\alpha_{2} \in B \circledast \alpha_{2}$. Thus $f\left(\alpha_{1}\right)=\alpha_{1}=\alpha_{2}=f\left(\alpha_{2}\right)$. If $\alpha_{1} \notin B \circledast \alpha_{1}$, then $\alpha_{2} \notin B \circledast \alpha_{2}$. Thus $f\left(\alpha_{1}\right)=r\left(\alpha_{1}\right)$ and $f\left(\alpha_{2}\right)=r\left(\alpha_{2}\right) . r$ is a function. Thus, from $\alpha_{1}=\alpha_{2}$ it follows that $r\left(\alpha_{1}\right)=r\left(\alpha_{2}\right)$. Therefore, $f\left(\alpha_{1}\right)=f\left(\alpha_{2}\right)$.

That $f$ satisfies lower boundary follows by the definition of $f$ and $\circledast$-stability.

We will now show that $f$ satisfies consistency preservation. Assume that $\forall \neg \alpha$. If $\alpha \in B \circledast \alpha$, then $f(\alpha)=\alpha$ from which it follows that $\forall \neg f(\alpha)$. Consider now that $\alpha \notin B \circledast \alpha$. By the definition of $f$ it follows that $f(\alpha) \in B \circledast \alpha$. On the other hand, by $\circledast$-consistency it follows that $B \circledast \alpha H \perp$. Therefore $\{f(\alpha)\} \forall \perp$. Hence $\forall \neg f(\alpha)$.

That $f$ satisfies implication follows from the definition of $f$.

To show that $f$ satisfies idempotence:

case 1) $\alpha \in B \circledast \alpha$. Thus $f(\alpha)=\alpha$. Hence $f(f(\alpha))=f(\alpha)$.

case 2) $\alpha \notin B \circledast \alpha$. Thus $f(\alpha)=r(\alpha)$ and $r(\alpha) \in B \circledast r(\alpha)$. Hence, from the definition of $f$, it follows that $f(r(\alpha))=r(\alpha)$. From the latter and $f(\alpha)=r(\alpha)$ it follows that $f(f(\alpha))=f(\alpha)$. We will now show that $f$ satisfies uniform identity. Assume that it holds for all subsets $B^{\prime} \subseteq B, B^{\prime} \cup\{\alpha\} \vdash \perp$ iff $B^{\prime} \cup\{\beta\} \vdash \perp$. Let $f(\alpha)=\alpha$. From the definition of $f$ it holds that $f(\alpha) \in B \circledast \alpha$. Thus $\alpha \in B \circledast \alpha$. By $\circledast$-uniform success it follows that $\beta \in B \otimes \beta$. From which it follows that $f(\beta)=\beta$. By symmetry of the case it follows that if $f(\beta)=\beta$, then $f(\alpha)=\alpha$. Hence it holds that $f(\alpha)=\alpha$ iff $f(\beta)=\beta$.

(b) That * satisfies success follows trivially from the definition of *. That * satisfies consistency follows from the fact that both $\circledast$ and $*^{\prime}$ satisfy consistency. Next we show that * satisfies inclusion.

If $\alpha \in B \circledast \alpha$, then by $\circledast$-weak inclusion it follows that $B \circledast \alpha \subseteq B \cup\{\alpha\}$. From which it follows, from the definition of *, that $B * \alpha \subseteq B \cup\{\alpha\}$. If $\alpha \notin B \circledast \alpha$, then $B * \alpha=B *^{\prime} \alpha$. It holds that $*^{\prime}$ satisfies inclusion thus $B * \alpha \subseteq B \cup\{\alpha\}$.

Relevance follows from the definition of $*$, $\circledast$-weak relevance and $*^{\prime}$-relevance.

For uniformity, assume that it holds for all subsets $B^{\prime} \subseteq B, B^{\prime} \cup\{\alpha\} \vdash \perp$ iff $B^{\prime} \cup\{\beta\} \vdash \perp$. By $\circledast$-uniform success it follows that $\alpha \in B \circledast \alpha$ iff $\beta \in B \circledast \beta$. We will consider two cases:

case 1) $\alpha \in B \circledast \alpha$. Then $\beta \in B \otimes \beta$. Thus $B * \alpha=B \circledast \alpha$ and $B * \beta=B \circledast \beta$. From which it follows by $\circledast$-weak uniformity that $B \cap B * \alpha=B \cap B * \beta$.

case 2) $\alpha \notin B \circledast \alpha$. Then $\beta \notin B \circledast \beta$. Thus $B * \alpha=B *^{\prime} \alpha$ and $B * \beta=B *^{\prime} \beta$. Hence by $*^{\prime}$-uniformity it follows that $B \cap B * \alpha=B \cap B * \beta$.

(c) We will now prove that $B \circledast \alpha=B * f(\alpha)$.

case 1) $\alpha \notin B \circledast \alpha$. From the definition of $f$ it holds that $B \circledast \alpha=B \circledast f(\alpha)$ and $f(\alpha) \in B \circledast \alpha$. Hence $f(\alpha) \in B \circledast f(\alpha)$. Thus, from the definition of $*$ it follows that $B * f(\alpha)=B \circledast f(\alpha)$, from which it follows that $B \circledast \alpha=B * f(\alpha)$.

case 2) $\alpha \in B \circledast \alpha$. Hence $f(\alpha)=\alpha$ and $B * \alpha=B \circledast \alpha$. Thus $B * f(\alpha)=B \circledast \alpha$.

(b) implies (a):

That $\circledast$ satisfies weak inclusion, consistency, weak uniformity, proxy success, stability, uniform success and weak relevance follows from Observation 12.

\section{Proof of Theorem 52}


Proof. Since this theorem is a slight variant of the previous one, substituting proxy success by weak proxy success in the postulates for $\circledast$, the proof is essentially the same. For this purpose, $f$ is not required to satisfy implication, as shown in Observation 12.

\section{Proof of Theorem 53}

Proof. (a) implies (b):

We will use essentially, for this part of the proof, the same constructions as in the proof of Theorem 51 . The only difference is that, in this proof, the operator $*^{\prime}$ used in the definition of * is a kernel base revision instead of a partial meet base revision operator as in the proof of that theorem (hence, according to Theorem $3, *^{\prime}$ satisfies inclusion, success, consistency, uniformity and core-retainment). The proof, for this part, is very similar to the one presented for Theorem 51. The only difference is that we need to show that * satisfies core-retainment instead of relevance. That * satisfies core-retainment follows from the definition of $*$, $\circledast$-weak core-retainment and $*^{\prime}$-core-retainment.

(b) implies (a):

That $\circledast$ satisfies weak inclusion, consistency, weak uniformity, proxy success, stability, uniform success and weak core-retainment follows from Observation 12.

\section{Proof of Theorem 55}

Proof. (1) implies (2):

We will use essentially, for this part of the proof, the same constructions as in the proof of Theorem 51 . The only difference is that, in this proof, the operator $*^{\prime}$ used in the definition of $*$ is a negation free partial meet revision instead of a revision operator as in the proof of the former theorem.

The proof that $f$ is a (well-defined) function that satisfies the properties listed in statement (2), * satisfies inclusion, success, consistency and relevance (by Theorem 5) and that $B \circledast \alpha=B * f(\alpha)$, for all $\alpha$, is essentially the same, just substituting $\forall \neg \alpha$ by $\alpha \forall \perp$. It remains to prove that * satisfies pre-expansion. There are two cases. If $\alpha \notin B \circledast \alpha$, it is valid because $\star^{\prime}$ satisfies pre-expansion. If $\alpha \in B \circledast \alpha$, it is also valid because $\circledast$ satisfaz weak pre-expansion.

(2) implies (1):

That $\circledast$ satisfies weak inclusion, consistency, weak uniformity, proxy success, stability, uniform success and weak relevance follows from Observation 12. It remains to prove that $\circledast$ satisfies weak pre-expansion.

(I) As $\alpha \in(B \cup\{\alpha\})$ then, by $f$-lower boundary, $f(\alpha)=\alpha$. So $(B \cup\{\alpha\}) \circledast \alpha=(B \cup\{\alpha\})$ * $f(\alpha)=(B \cup\{\alpha\}) * \alpha$.

(II) $B \circledast \alpha=B * f(\alpha)$. Suppose that $\alpha \in B \circledast \alpha$. Then $\alpha \in B * f(\alpha)$. There are two cases. If $\alpha \in B$, by $f$-lower boundary we have that $f(\alpha)=\alpha$, which implies that $B * f(\alpha)=B * \alpha$. If $\alpha \notin B$, as $\alpha \in B * f(\alpha)$, by *-inclusion and success we have that $f(\alpha)=\alpha$ and $B * f(\alpha)=B * \alpha$. By *-pre-expansion, it follows that $(B \cup\{\alpha\}) * \alpha=B * \alpha$. Therefore, if $\alpha \in B \circledast \alpha$, $(B \cup\{\alpha\}) \circledast \alpha=B \circledast \alpha$, which proves weak pre-expansion. 


\section{Proof of Observation 13}

Proof. 1. We prove by cases: (a) If $K \cup A \vdash \perp$, then $C n(K \cup A)=K_{\perp}$ and, therefore, $K \odot A \subseteq C n(K \cup A)$. (b) If $K \cup A H \perp$, then $K \odot A=K{ }^{*} f(A)$ and, by ${ }^{*}$-inclusion, $K{ }_{p} f(A) \subseteq C n(K \cup f(A))$. By weak implication we have that $C n(K \cup f(A)) \subseteq C n(K \cup A)$. Hence, $K \odot A \subseteq C n(K \cup A)$.

2. Since $f(A) \forall \perp$, by ${ }^{*}$ - weak consistency we have that $K{ }^{*} p(A) \forall \perp$. Thus, $K \odot A \forall \perp$.

3. Trivial, since by definition ${ }^{*}$ s satisfies success and by maximality $K \odot A=K{ }^{*} p f(A)=$ $K *{ }_{p} A$.

4. If $A \forall \perp$ then $f(A) \forall \perp$ and, by ${ }^{*}$-weak consistency, $K \odot A=K{ }^{*} f(A) \forall \perp$.

\section{Proof of Observation 14}

Proof. Assume that $A \subseteq K \odot A$. Since $K \odot A=K *_{p} f(A)$, we have that $A \subseteq K *_{p} f(A)$ and, by *-inclusion, $A \subseteq C n(K \cup f(A))$. Then we have three possibilities: $(i) A \subseteq K$, which implies that $A \backslash K=\varnothing \subseteq C n(f(A))$. (ii) $A \subseteq C n(f(A))$, which also implies that $A \backslash K \subseteq C n(f(A))$. (iii) $A \cap K \neq \varnothing$ and $A \backslash K \subseteq C n(f(A))$. By $f$-conditional maximality, in all of the three cases we have that $C n(f(A))=C n(A)$.

\section{Proof of Theorem 59}

Proof. (1) implies (2): we first define $f$ and ${ }^{*}$ :

$$
f(A)= \begin{cases}A & \text { if } K \cup A H \perp \text { or } A \subseteq K \odot A ; \\ r(A) & \text { otherwise, where } r \text { is a (well-defined) function } \mathcal{P}(\mathcal{L}) \times \mathcal{P}(\mathcal{L}) \longrightarrow \\ & \mathcal{P}(\mathcal{L}) \text { such that } r(A) \subseteq C n(K \odot A), K \odot A=K \odot r(A) \text { and for all } \\ & A \text { and } A^{\prime} \text { such that } K \odot A=K \odot A^{\prime}, r(A)=r\left(A^{\prime}\right) .\end{cases}
$$

This definition is possible since $\odot$ satisfies weak proxy success.

$$
K * A= \begin{cases}K \odot A & \text { if } A \subseteq C n(K \odot A) ; \\ K *_{p}^{\prime} A & \text { otherwise, where } *_{p}^{\prime} \text { is any operation that satisfies the six axioms } \\ & \text { from Observation } 7 .\end{cases}
$$

We need to show that:

(a) $f$ is a (well-defined) transformation function;

(b) $f$ satisfies the properties;

(c) * is a multiple theory revision (see Observation 7);

(d) $K \odot A=K * f(A)$, for all $A$. 
The proofs are given below:

(a) To prove that $f$ is a (well defined) function we must show that for all $A \subseteq \mathcal{L}$ there exists $A^{\prime} \subseteq \mathcal{L}$ such that $f(A)=A^{\prime}$ and that, if $A_{1}=A_{2}$, then $f\left(A_{1}\right)=f\left(A_{2}\right)$.

Let $A \subseteq \mathcal{L}$. If $K \cup A \forall \perp$ or $A \subseteq K \odot A$, then $f(A)=A$. Otherwise, $f(A)=r(A)=A^{\prime}$, for some $A^{\prime}$ such that $A^{\prime} \subseteq K \odot A=K \odot A^{\prime}$. Such $A^{\prime}$ exists since $\odot$ satisfies weak proxy success and closure. Assume now that $A_{1}=A_{2}$. If $K \cup A_{1} \forall \perp$, then $K \cup A_{2} \forall \perp$, or if $A_{1} \subseteq K \odot A_{1}$, then $A_{2} \subseteq K \odot A_{2}$. Thus, in both cases $f\left(A_{1}\right)=A_{1}=A_{2}=f\left(A_{2}\right)$. If $K \cup A_{1} \vdash \perp$ and $A_{1} \nsubseteq K \odot A_{1}$, then $K \cup A_{2} \vdash \perp$ and $A_{2} \nsubseteq K \odot A_{2}$. Thus, $f\left(A_{1}\right)=r\left(A_{1}\right)$ and $f\left(A_{2}\right)=r\left(A_{2}\right) . r$ is a (well-defined) function. Hence, from $A_{1}=A_{2}$ it follows that $r\left(A_{1}\right)=r\left(A_{2}\right)$. Therefore, $f\left(A_{1}\right)=f\left(A_{2}\right)$.

(b) That $f$ satisfies weak maximality follows from the definition of $f$. To show that $f$ satisfies consistency preservation, let $A \forall \perp$; if $K \cup A H \perp$ or $A \subseteq K \odot A$, then $f(A)=A$ and $f(A) \forall \perp$; otherwise, then $f(A)=r(A)$ and $r(A) \subseteq C n(K \odot A)$; since $A \forall \perp$, by $\odot$-consistency $K \odot A \forall \perp$, which implies that $r(A) \forall \perp$ and, finally, that $f(A) \forall \perp$.

To show that $f$ satisfies uniformity suppose that for every $K^{\prime} \subseteq K$ it holds that $K^{\prime} \cup A \vdash \perp$ iff $K^{\prime} \cup C \vdash \perp$. If $K \cup A H \perp$, then $f(A)=A$ and $K \cup C H \perp$; hence, $f(C)=C$. If $A \subseteq K \odot A$ then, by $\odot$-uniform success, $C \subseteq K \odot C$ and, hence, $f(A)=A$ and $f(C)=C$; thus, in both cases it holds that $K^{\prime} \cup f(A) \vdash \perp$ iff $K^{\prime} \cup f(C) \vdash \perp$. If $K \cup A \vdash \perp$ and $A \nsubseteq K \odot A$, then by $\odot$-uniform success and the initial assumption it holds that $K \cup C \vdash \perp$ and $C \nsubseteq K \odot C$. Thus, $f(A)=r(A)$ and $f(C)=r(C)$. By $\odot$-uniformity, $K \odot A=K \odot C$ and, from the definition of $r$, it follows that $r(A)=r(C)$. Therefore, $K^{\prime} \cup f(A) \vdash \perp$ iff $K^{\prime} \cup f(C) \vdash \perp$.

To show that $f$ satisfies uniform identity, assume that for every $K^{\prime} \subseteq K$ it holds that $K^{\prime} \cup A \vdash \perp$ iff $K^{\prime} \cup C \vdash \perp$. Let $C n(f(A)=C n(A)$. From the definition of $f$ it holds that $f(A) \subseteq K \odot A$. Thus, by ${ }^{*}$-closure it holds that $A \subseteq K \odot A$. By $\odot$-uniform success it follows that $C \subseteq K \odot C$, from which follows that $f(C)=C$ and, hence, $C n(f(C))=C n(C)$. By symmetry of the case, it follows that if $C n(f(C))=C n(C)$, then $C n(f(A))=C n(A)$. Hence, it holds that $C n(f(A))=C n(A)$ iff $C n(f(C))=C n(C)$.

To show that $f$ satisfies lower boundary, assume that $A \subseteq K$. From $\odot$-stability it follows that $A \subseteq K \odot A$ and, by the definition of $f, f(A)=A$.

To show that $f$ satisfies conditional maximality, assume that $A \backslash K \subseteq C n(f(A))$. From the definition of $f$ we have that $f(A) \subseteq K \odot A$, which implies by * ${ }_{p}$-closure that $A \backslash K \subseteq K \odot A$. Then, by $\odot$-conditional success, $A \subseteq K \odot A$ and, from the definition of $f$, it holds that $f(A)=A$ and, hence, $C n(f(A))=C n(A)$.

Finally, we show that $f$ satisfies idempotence. If $K \cup A \forall \perp$ or $A \subseteq K \odot A$ then, from the definition of $f, f(f(A))=f(A)$ follows directly. Otherwise, $f(A)=r(A)$ and, by the definition of $r, r(A) \subseteq K \odot r(A)$. From the definition of $f$, since $r(A) \subseteq K \odot r(A)$, we have that $f(r(A))=r(A)$ and, given that $f(A)=r(A)$, we have that $f(f(A))=$ $f(r(A)=r(A)=f(A)$.

(c) In order to show that $*$ is a multiple package theory revision, we need to prove that it satisfies the six axioms from Observation 7. That * satisfies closure, inclusion, uniformity and weak consistency is trivial, since both $\odot$ and $*^{\prime}$ satisfy these four 
postulates. That * satisfies success also follows directly from the definition. In order to show that * satisfies relevance, if $A \nsubseteq C n(K \odot A)$, we are done (given that $*_{p}^{\prime}$ satisfies relevance). If $A \subseteq C n(K \odot A)$, then $K * A=K \odot A$. Suppose that $\exists \beta \in K$ such that $\beta \notin K \odot A$. By $\odot$-vacuity, $K \cup A \vdash \perp$. By $\odot$-weak relevance, $\exists K^{\prime}$ such that $(K \odot A) \cap K \subseteq K^{\prime} \subseteq K, K^{\prime} \cup A H \perp$ and $K^{\prime} \cup\{\beta\} \cup A \vdash \perp$. Therefore, as $K * A=K \odot A$, we can conclude that relevance is satisfied.

(d) We need to prove that $K \odot A=K * f(A)$. If $K \cup A H \perp$ or $A \subseteq K \odot A, f(A)=A$ and $K \odot f(A)=K \odot A$. In the case of $K \cup A H \perp$, by $\odot$-vacuity it follows that $A \subseteq K \odot A$ and, then, $f(A) \subseteq K \odot f(A)$. By the definition of $*, K * f(A)=K \odot f(A)$. Hence, $K * f(A)=K \odot A$. If $K \cup A \vdash \perp$ and $A \nsubseteq K \odot A$ then it follows from the definitions of $f$ and $r$ that $f(A) \subseteq C n(K \odot A)$ and $K \odot A=K \odot f(A)$, from which follows that $f(A) \subseteq C n(K \odot f(A))$. Then, from the definition of $*$ it follows that $K * f(A)=K \odot f(A)=K \odot A$.

(2) implies (1): That $\odot$ satisfies closure is trivial since by Observation $7{ }^{*} p$ satisfies closure.

In order to prove weak proxy success, we have that by Definition 91 and idempotence, $K \odot A=K *_{p} f(A)=K *_{p} f(f(A))=K \odot f(A)$. We therefore have $f(A) \subseteq C n\left(K *_{p} f(A)\right)$, $K \odot A=K \odot f(A)$ and $f(A) \subseteq C n(K \odot A)$, which is sufficient to prove that $\odot$ satisfies weak proxy success.

In order to prove inclusion, weak maximality implies weak implication; then, inclusion follows from item 1 of Observation 13.

For vacuity, suppose that $K \cup A H \perp$. Then, by $f$-weak maximality, $C n(f(A))=C n(A)$. Since $K \odot A=K *_{p} f(A)$, by * ${ }_{p}$-success $f(A) \subseteq K *_{p} f(A)$ and, by $*_{p}$-closure, $C n(f(A)) \subseteq$ $K{ }^{*} p(A)$. Since $C n(f(A))=C n(A)$, we also have that $C n(A) \subseteq K{ }^{*} p(A)$. From $K \cup A \forall \perp$ and $C n(f(A))=C n(A)$, we have that $K \cup f(A) H \perp$. Thus, by ${ }^{*}$-vacuity, $K{ }^{*} p f(A)=$ $C n(K \cup f(A))=C n(K \cup A)$. Therefore, $K \odot A=C n(K \cup A)$.

For weak consistency, let $A \forall \perp$. Then, by consistency preservation we have that $f(A) \forall \perp$ and, by * ${ }_{p}$-weak consistency, we have that $K{ }^{*} p f(A) H \perp$. Thus, $K \odot A H \perp$.

In order to prove uniformity, assume that for every $K^{\prime} \subseteq K$ it holds that $K^{\prime} \cup A \vdash \perp$ iff $K^{\prime} \cup C \vdash \perp$. Then, by $f$-uniformity, it holds that $K^{\prime} \cup f(A) \vdash \perp$ iff $K^{\prime} \cup f(C) \vdash \perp$ and, by ${ }^{*}{ }_{p}$-uniformity, $K{ }^{*} p f(A)=K{ }_{p} f(B)$ or, equivalently, $K \odot A=K \odot B$.

For uniform success, assume that for every $K^{\prime} \subseteq K$ it holds that $K^{\prime} \cup A \vdash \perp$ iff $K^{\prime} \cup C \vdash \perp$. By $f$-uniform identity we have that $C n(f(A))=C n(A)$ iff $C n(f(C))=C n(C)$. Let $A \subseteq K \odot A$. From $f$-conditional maximality and Observation 14 we have that $C n(f(A))=C n(A)$. By $f$-uniform identity it follows that $C n(f(C))=C n(C)$. From the definition of $\odot$ it holds that $K \odot C=K{ }^{*} f(C)$. By *-success we have that $f(C) \subseteq K \odot C$ and, by *-closure, $C \subseteq K \odot C$. By symmetry of the case it holds that if $C \subseteq K \odot C$, then $A \subseteq K \odot A$. Hence it holds that $A \subseteq K \odot A$ iff $C \subseteq K \odot C$.

In order to prove conditional success, let $A \backslash K \subseteq K \odot A$. Since $K \odot A=K *_{p} f(A)$, by ${ }^{*}$ - -inclusion we have that $A \backslash K \subseteq C n(K \cup f(A))$, which implies that $A \backslash K \subseteq C n(f(A))$. From $f$-conditional maximality we have that $C n(f(A))=C n(A)$ and, by ${ }^{*} p$-success and ${ }_{p} p$-closure, $A \subseteq K{ }^{*} f(A)$. Thus, $A \subseteq K \odot A$. 
For stability, let $A \subseteq K$. Then, since $f$ satisfies lower boundary, we have that $C n(f(A))=$ $C n(A)$, which implies that, for any set $X, X \cup f(A) \vdash \perp$ iff $X \cup A \vdash \perp$ and, by ${ }_{p}$-uniformity $K{ }^{*} f(A)=K{ }_{p} A$. Since $K \odot A=K{ }_{p} f(A)$, it holds that $K \odot A=K{ }^{*} A$. From ${ }_{p}$-success it follows that $A \subseteq K{ }_{p} A=K \odot A$.

It only remains to prove that $\odot$ satisfies weak relevance. Suppose that $A \subseteq K \odot A$ and $\exists \beta \in K$ such that $\beta \notin K \odot A$. As $K \odot A=K{ }^{*} p f(A)$, we have that $\beta \notin K{ }^{*} p f(A)$. By ${ }^{*}{ }_{p}$-relevance, $\exists K^{\prime}$ such that $\left(K{ }_{p} f(A)\right) \cap K \subseteq K^{\prime} \subseteq K, K^{\prime} \cup f(A) \forall \perp$ but $K^{\prime} \cup\{\beta\} \cup f(A) \vdash \perp$. Since $K \odot A=K{ }^{*} f(A),(K \odot A) \cap K \subseteq K^{\prime} \subseteq K$. So, it remains to prove that $K^{\prime} \cup A \forall \perp$. Given that $A \subseteq K \odot A$, then $A \subseteq K{ }^{*} p f(A)$ and, by ${ }_{p}$-inclusion we have three possibilities. If $A \subseteq K$, by $f$-lower boundary we have that $C n(f(A))=C n(A)$, and also if $A \subseteq f(A)$. If $((K \odot A) \cap K) \cap A \neq \varnothing \neq A \cap f(A)$, we can conclude that, since $(K \odot A) \cap K \subseteq K^{\prime}, A \subseteq K^{\prime} \cup f(A)$. Therefore, $K^{\prime}$ is such that $K^{\prime} \cup A H \perp$ but $K^{\prime} \cup\{\beta\} \cup A \vdash \perp$. Thus, $\odot$-weak relevance is satisfied.

\section{Proof of Theorem 60}

Proof. This proof is quite similar to that of Theorem 59. To show that (1) implies (2), we define $f$ to be a function like that of the previous proof but with an additional restriction when $K \cup A \vdash \perp$ and $A \nsubseteq K \odot A: f(A) \subseteq C n(A)$. The existence of such a function follows from proxy success. The proofs for $f$ are essentially the same, and the implication property follows trivially. To show that (2) implies (1) we only have to add a proof for proxy success, which we obtain from Theorem 59 and $f$-implication.

\section{Proof of Theorem 61}

Proof. This proof is quite similar to that of Theorem 59. To show that (1) implies (2), we define $f$ to be a function like that of the previous proof but with an additional restriction when $K \cup A \vdash \perp$ and $A \nsubseteq K \odot A: f(A) \subseteq A$. The existence of such a function follows from choice success. The proofs for $f$ are essentially the same, and the choice property follows trivially. To show that (2) implies (1) we only have to add a proof of choice success, which we obtain from Theorem 59 and $f$-choice.

\section{Proof of Observation 15}

Proof. 1. We prove by cases: (a) If $B \cup A \vdash \perp$, then $C n(B \cup A)=K_{\perp}$ and, therefore, $B \odot A \subseteq C n(B \cup A)$. (b) If $B \cup A H \perp$, then $B \odot A=B *_{p} f(A), B *_{p} f(A) \subseteq B \cup f(A)$ ( ${ }^{*}$-inclusion), $B \cup f(A) \subseteq B \cup C n(A)$ (weak implication). Hence, $B \odot A \subseteq C n(B \cup A)$.

2. Let $A \forall \perp$. Then, by $f$-consistency preservation, $f(A) \forall \perp$ and, by ${ }_{p}$-weak consistency, $B *_{p} f(A) H \perp$. Therefore, $B \odot A H \perp$ and weak consistency is satisfied.

3. We have that $B \odot A=B *_{p} f(A)$; then by $*_{p}$-inclusion $B *_{p} f(A) \subseteq B \cup f(A)$ and, since $f$ satisfies implication, $B *_{p} f(A) \subseteq B \cup C n(A)$. Thus, $B \odot A \subseteq B \cup C n(A)$ and weak inclusion is satisfied. That $\odot$ satisfies weak consistency follows from item 2 , since implication implies consistency preservation.

4. We have that $B \odot A=B *_{p} f(A)$; then by ${ }_{p}$-inclusion $B *_{p} f(A) \subseteq B \cup f(A)$ and, since $f$ satisfies choice, $B{ }^{*} p(A) \subseteq B \cup A$. Thus, $B \odot A \subseteq B \cup A$ and inclusion is satisfied. 
5. Weak maximality implies weak implication; then, very weak inclusion follows from item 1 . For vacuity, suppose that $B \cup A \forall \perp$. Then, by weak maximality, $A=f(A)$ so that $B \odot A=B{ }^{*} p A$ and, by ${ }_{p}$-vacuity, $B \odot A=B{ }_{p} A=B \cup A$.

\section{Proof of Observation 16}

Proof. Assume that $A \subseteq B \odot A$. Since $B \odot A=B{ }^{*} f(A)$, we have that $A \subseteq B{ }^{*} p f(A)$ and, by *-inclusion, $A \subseteq B \cup f(A)$. Then we have three possibilities: (i) $A \subseteq B$, which implies that $A \backslash B=\varnothing \subseteq f(A)$. (ii) $A \subseteq f(A)$, which also implies that $A \backslash B \subseteq f(A)$. (iii) $A \cap B \neq \varnothing$ and $A \backslash B \subseteq f(A)$. By $f$-conditional maximality, in all of the three cases we have that $f(A)=A$.

\section{Proof of Theorem 62}

Proof. (1) implies (2): we first define $f$ and *:

$$
f(A)= \begin{cases}A & \text { if } A \subseteq B \odot A ; \\ r(A) & \text { otherwise, where } r \text { is a (well defined) function } \mathcal{P}(\mathcal{L}) \times \mathcal{P}(\mathcal{L}) \longrightarrow \\ & \mathcal{P}(\mathcal{L}) \text { such that } r(A) \subseteq B \odot A \text { and } B \odot A=B \odot r(A) .\end{cases}
$$

This definition is possible since $\odot$ satisfies weak proxy success.

$$
B * A= \begin{cases}B \odot A & \text { if } A \subseteq B \odot A ; \\ B *^{\prime} A & \text { otherwise, where } *^{\prime} \text { is any operation that satisfies the five ax- } \\ & \text { ioms from Theorem } 15\end{cases}
$$

We need to show that:

(a) $f$ is a (well-defined) transformation function;

(b) $f$ satisfies the properties;

(c) * is a multiple base revision, according to Theorem 15;

(d) $B \odot A=B * f(A)$, for all $A$.

The proofs are given below:

(a) To prove that $f$ is a (well defined) function we must show that for all $A \subseteq \mathcal{L}$ there exists $A^{\prime} \subseteq \mathcal{L}$ such that $f(A)=A^{\prime}$ and that, if $A_{1}=A_{2}$, then $f\left(A_{1}\right)=f\left(A_{2}\right)$.

Let $A \subseteq \mathcal{L}$. If $A \subseteq B \odot A$, then $f(A)=A$. Otherwise, $f(A)=r(A)=A^{\prime}$, for some $A^{\prime}$ such that $A^{\prime} \subseteq B \odot A=B \odot A^{\prime}$. Such $A^{\prime}$ exists since $\odot$ satisfies weak proxy success. Assume now that $A_{1}=A_{2}$. If $A_{1} \subseteq B \odot A_{1}$, then by $\odot$-uniform success it follows that $A_{2} \subseteq B \odot A_{2}$. Thus $f\left(A_{1}\right)=A_{1}=A_{2}=f\left(A_{2}\right)$. If $A_{1} \nsubseteq B \odot A_{1}$, then $A_{2} \nsubseteq B \odot A_{2}$. Thus 
$f\left(A_{1}\right)=r\left(A_{1}\right)$ and $f\left(A_{2}\right)=r\left(A_{2}\right) . r$ is a (well-defined) function. Thus, from $A_{1}=A_{2}$ it follows that $r\left(A_{1}\right)=r\left(A_{2}\right)$. Therefore, $f\left(A_{1}\right)=f\left(A_{2}\right)$.

(b) To show that $f$ satisfies conditional maximality, assume that $A \backslash B \subseteq f(A)$. From the definition of $f$ we have that $f(A) \subseteq B \odot A$, which implies that $A \backslash B \subseteq B \odot A$. Then, by $\odot$ conditional success, $A \subseteq B \odot A$ and, from the definition of $f$, we have that $f(A)=A$. We will now show that $f$ satisfies consistency preservation. Assume that $A \Vdash \perp$. If $A \subseteq B \odot A$, then $f(A)=A$, from which it follows that $f(A) \forall \perp$. If $A \nsubseteq B \odot A$, then from the definition of $f$ it follows that $f(A) \subseteq B \odot A$. By $\odot$ weak consistency it follows that $B \odot A \forall \perp$ and, hence, $f(A) \forall \perp$.

To show that $f$ satisfies idempotence, we prove by cases:

(1) $A \subseteq B \odot A$. Thus $f(A)=A$ and $f(f(A))=f(A)$.

(2) $A \nsubseteq B \odot A$. Thus $f(A)=r(A)$ and $r(A) \subseteq B \odot r(A)$. Hence, from the definition of $f$ it follows that $f(r(A))=r(A)$. From the latter and $f(A)=r(A)$ it follows that $f(f(A))=f(A)$.

We will now show that $f$ satisfies uniform identity. Assume that it holds for all subsets $B^{\prime} \subseteq B, B^{\prime} \cup A \vdash \perp$ iff $B^{\prime} \cup C \vdash \perp$. Let $f(A)=A$. From the definition of $f$ it holds that $f(A) \subseteq B \odot A$. Thus $A \subseteq B \odot A$. By $\odot$ uniform success it follows that $C \subseteq B \odot C$, from which follows that $f(C)=C$. By symmetry of the case it follows that if $f(C)=C$, then $f(A)=A$. Hence it holds that $f(A)=A$ iff $f(C)=C$.

(c) That * satisfies success follows trivially from definition of *. That * satisfies weak consistency and inclusion follows from the fact that both $\odot$ and $*^{\prime}$ satisfy weak consistency and inclusion.

Relevance follows from the definition of $*, \odot$ weak relevance and $*^{\prime}$ relevance.

In order to show that * satisfies uniformity, assume that it holds for all subsets $B^{\prime} \subseteq B, B^{\prime} \cup A \vdash \perp$ iff $B^{\prime} \cup C \vdash \perp$. By $\odot$ uniform success it follows that $A \subseteq B \odot A$ iff $C \subseteq B \odot C$. We prove by cases:

(1) $A \subseteq B \odot A$. Then $C \subseteq B \odot C$ and, hence, $B * A=B \odot A$ and $B * C=B \odot C$, from which it follows by $\odot$ conditional uniformity that $B \cap(B * A)=B \cap(B * C)$.

(2) $A \nsubseteq B \odot A$. Then $C \nsubseteq B \odot C$. Thus $B * A=B *^{\prime} A$ and $B * C=B *^{\prime} C$. Hence by $*^{\prime}$ uniformity it follows that $B \cap(B * A)=B \cap(B * C)$.

(d) We will now prove that $B \odot A=B * f(A)$.

case 1) $A \subseteq B \odot A$. Hence $f(A)=A$ and $B * A=B \odot A$. Thus $B * f(A)=B \odot A$.

case 2) $A \nsubseteq B \odot A$. From the definition of $f$ it holds that $B \odot A=B \odot f(A)$ and $f(A) \subseteq B \odot A$. Hence $f(A) \subseteq B \odot f(A)$. Thus, from the definition of * it follows that $B * f(A)=B \odot f(A)$, from which it follows that $B \odot A=B * f(A)$.

(2) implies (1): For conditional success, let $A \backslash B \subseteq B \odot A$. Since $B \odot A=B{ }^{*} f(A)$, by * $p$-inclusion we have that $A \backslash B \subseteq B \cup f(A)$, which implies that $A \backslash B \subseteq f(A)$. From $f$-conditional maximality we have that $f(A)=A$ and, by ${ }^{*}$-success, it follows that $A \subseteq B * A$. Thus $A \subseteq B \odot A$.

In order to prove conditional inclusion, let $A \subseteq B \odot A$. Since $f$ satisfies conditional maximality, from Observation 16 we have that $f(A)=A$ and, since $B \odot A=B{ }^{*} f(A)$, by *-inclusion we have that $B \odot A \subseteq B \cup A$. 
For weak relevance, let $A \subseteq B \odot A$ and suppose that there is some $\beta \in B$ such that $\beta \notin B \odot A$. Since $B \odot A=B *_{p} f(A)$, we have that $\beta \in B \backslash\left(B *_{p} f(A)\right)$ and, by $*_{p}$-relevance, there is a set $B^{\prime}$ such that $B{ }^{*} f(A) \subseteq B^{\prime} \subseteq B \cup f(A), B^{\prime} H \perp$ but $B^{\prime} \cup\{\beta\} \vdash \perp$. So it remains to prove that $B^{\prime} \subseteq B \cup A$. Since from $A \subseteq B \odot A$ and $f$-conditional maximality we have from Observation 15 that $f(A)=A$, it follows directly.

Weak consistency follows from item 2 of Observation 15.

For uniform success, consider that it holds for all subsets $B^{\prime}$ of $B$ that $B^{\prime} \cup A \vdash \perp$ iff $B^{\prime} \cup C \vdash \perp$. Hence, since $f$ satisfies uniform identity, $f(A)=A$ holds iff $f(C)=C$ holds. Assume that $A \subseteq B \odot A$. From $f$-conditional maximality and Observation 16 we have that $f(A)=A$. On the other hand, by $f$-uniform identity it follows that $f(C)=C$. From the definition of $\odot$ it holds that $B \odot C=B{ }_{p} f(C)$. By * ${ }_{p}$-success it follows that $C=f(C) \subseteq B \odot C$. Thus $C \subseteq B \odot C$. By symmetry of the case it holds that if $C \subseteq B \odot C$, then $A \subseteq B \odot A$. Hence it holds that $A \subseteq B \odot A$ iff $C \subseteq B \odot C$.

In order to show that $\odot$ satisfies conditional uniformity, consider that it holds for all subsets $B^{\prime}$ of $B$ that $B^{\prime} \cup A \vdash \perp$ iff $B^{\prime} \cup C \vdash \perp$. Let $A \subseteq B \odot A$. From $f$-conditional maximality and Observation 16 we have that $f(A)=A$ and from $f$-uniform identity it follows that $f(C)=C$. By $*_{p}$-uniformity it follows that $B \cap\left(B *_{p} A\right)=B \cap\left(B *_{p} C\right)$. Thus $B \cap(B \odot A)=B \cap\left(B *_{p} f(A)\right)=B \cap\left(B *_{p} A\right)=B \cap\left(B *_{p} C\right)=B \cap\left(B *_{p} f(C)\right)=B \cap(B \odot C)$.

\section{Proof of Theorem 63}

Proof. This proof is quite similar to that of Theorem 64. To show that (1) implies (2), we define $f$ to be a function like that of the previous proof but with an additional restriction for $r: r(A) \subseteq C n(A)$. The existence of such a function follows from proxy success. The proofs for $f$ are essentially the same, and the implication property follows trivially. To show that (2) implies (1) we only have to add a proof of proxy success, which we obtain from Observation 15.

\section{Proof of Theorem 64}

Proof. This proof is quite similar to that of Theorem 62. To show that (1) implies (2), we define $f$ to be a function like that of the previous proof but with an additional restriction for $r: r(A) \subseteq A$. The existence of such a function follows from choice success. The proofs for $f$ are essentially the same, and the choice property follows trivially. To show that (2) implies (1) we only have to add proofs for inclusion and choice success, which we obtain from item 4 of Observation 15 and from Theorem 62 and $f$-choice. 



\section{Appendix B}

\section{Proofs related to Chapter 7}

\section{Proof of Theorem 65}

Proof. If $A \forall \perp$, both directions of the proof follow as in Theorem 35 since $\dot{+}$-success implies +-weak success.

Otherwise, from construction to postulates we have that $\dot{+}$-weak success is vacuously satisfied and, since $B \bowtie A=\varnothing, B+A=B$ and, hence, $\dot{+}$-inclusion and $\dot{+}$-preservation follow immediately. In order to show + -coincidence, let $B, A$ and $C$ be such that $B \cap A \neq \varnothing$ and $A \subseteq C \subseteq(B \cup A)$. Since $A \vdash \perp$, it follows that $C \vdash \perp$ and $B+A=B=B+C$. For the other direction, the selection function is defined as in the proof for Theorem 35 .

\section{Proof of Observation 17}

Proof. The item (a) can be trivially shown.

(b) First, it is easy to see that if it holds for all $B^{\prime} \subseteq B$ that $B^{\prime} \cup\{\alpha\} \vdash \perp$ for every $\alpha \in A$ iff $B^{\prime} \cup\{\psi\} \vdash \perp$ for every $\psi \in C$, then it holds for all $B^{\prime} \subseteq B$ that $B^{\prime} \cup\{\alpha\} \forall \perp$ for some $\alpha \in A$ iff $B^{\prime} \cup\{\psi\} \forall \perp$ for some $\psi \in C$. The converse property holds as well. (Uniformity 1 implies Uniformity 2): assume that the initial condition from $*_{c}$ uniformity 2 holds; then the initial condition from ${ }^{*}{ }_{c}$-uniformity 1 holds as well and, hence, $B \backslash\left(B *_{c} A\right)=B \backslash\left(B *_{c} C\right)$. We must show that $B \cap\left(B *_{c} A\right)=B \cap\left(B *_{c} C\right)$. By set properties, it holds that $B=\left(B \cap\left(B *_{c} A\right)\right) \cup\left(B \backslash\left(B *_{c} A\right)\right)$ where $\left(B \cap\left(B *_{c} A\right)\right) \cap\left(B \backslash\left(B *_{c} A\right)\right)=\varnothing$. Similarly, it holds that $B=\left(B \cap\left(B *_{c} C\right)\right) \cup\left(B \backslash\left(B *_{c} C\right)\right)$, where $\left(B \cap\left(B *_{c} C\right)\right) \cap\left(B \backslash\left(B *_{c} C\right)\right)=\varnothing$. Therefore, since $B \backslash\left(B *_{c} A\right)=B \backslash\left(B *_{c} C\right)$, we can conclude that $B \cap\left(B *_{c} A\right)=B \cap\left(B *_{c} C\right)$. (Uniformity 2 implies Uniformity 1): we must show that if $B \cap\left(B *_{c} A\right)=B \cap\left(B *_{c} C\right)$ then $B \backslash\left(B{ }^{*} A\right)=B \backslash\left(B{ }_{c} C\right)$. Using set properties, the proof is similar to the previous case.

(c) Let $*_{c}$ be an operator that satisfies core-retainment and assume that $B \cup A$ is consistent. We need to show that $B \subseteq B *_{c} A$. By reductio ad absurdum, suppose that $B \nsubseteq B *_{c} A$. Therefore, there is some $\varphi \in B$ such that $\varphi \notin B{ }^{*} A$. Due to core-retainment, there is a set $B^{\prime} \subseteq B$ such that $B^{\prime} \cup\{\psi\}$ is consistent for some $\psi \in A$ but $B^{\prime} \cup\{\varphi\} \cup\{\lambda\}$ is inconsistent for every $\lambda \in A$. But this is absurd because $B \cup A$ is consistent. Then, $B \subseteq B{ }^{*} A$. 
(d) Let $*_{c}$ be an operator that satisfies $*_{c}$-vacuity $1, *_{c}$-vacuity 2 and $*_{c}$-weak success. If $A=\varnothing$ or $A \vdash \perp$, then by $*_{c}$-vacuity 1 we have that $B *_{c} A=B$. Otherwise, we have from ${ }^{*}{ }_{c}$-weak success that $A \cap\left(B{ }_{c} A\right) \neq \varnothing$. Assume that $\left(A \cap\left(B{ }_{c}{ }_{c} A\right)\right) \subseteq B$. Then, $A \cap B \neq \varnothing$. By ${ }^{*_{c}}$-vacuity 2 we have that $B{ }_{c} A=B$, which satisfies ${ }^{*}{ }_{c}$-confirmation.

The item (e) is trivially shown.

\section{Proof of Observation 18}

Proof. If $B \cup\{\psi\} \forall \perp$ for some $\psi \in A$, then $B$ satisfies the second condition of the remainder set of $B$ by $A$ and the third one is vacuously satisfied. If $B \forall \perp$ and $B \cap A \neq \varnothing$, we have that $B \cup(B \cap A) \forall \perp$ and, given that $B \cap A \subseteq A, B$ satisfies the second condition of the remainder set of $B$ by $A$ (and the third one is again vacuously satisfied). Hence, in both cases $\{B\} \in B \downarrow_{c} A$ and any $B^{\prime} \subseteq B$ could not be part of the remainder set since it would not be maximal. Therefore, $B \downarrow_{c} A=\{B\}$.

\section{Proof of Observation 19}

Proof. Let $X \subseteq B$ be such that $X \in B \downarrow_{c} A$. Then $X$ is consistent with some $\alpha \in A$ and, thus, consistent with some $\psi \in C$. Suppose that $X \notin B \downarrow_{c} C$. From Definition 93, the only possible reason for that is $X$ not being maximal: there exists some $X^{\prime}$ such that $X \subset X^{\prime}$ and $X^{\prime} \in B \downarrow_{c} C$. As a consequence, $X^{\prime} \cup\{\alpha\}$ is consistent for some $\alpha \in A$ and $X \notin B \downarrow_{c} A$, since $X$ is not maximal. Contradiction. Therefore, if $X \in B \downarrow_{c} A$, then $X \in B \downarrow_{c} C$. The converse property is analogous. Therefore, $B \downarrow_{c} A=B \downarrow_{c} C$.

\section{Proof of Observation 20}

Proof. If $B \cap A=\varnothing$, we are done. Otherwise, since $B \forall \perp$, from Observation 18 we have that $B \downarrow_{c} A=\{B\}$ and, hence, $\bigcap \gamma\left(B \downarrow_{c} A\right)=B$. Therefore, $B \cap A \subseteq \bigcap \gamma\left(B \downarrow_{c} A\right)$.

\section{Proof of Observation 21}

Proof. Let $B$ and $A$ be belief bases such that $B H \perp$ or $B \cap A=\varnothing$. By the definitions of $B \downarrow_{c} A$ and $\gamma$ and the +-preservation property, $\bigcap \gamma\left(B \downarrow_{c} A\right) \subseteq B$ and $\bigcap \gamma\left(B \downarrow_{c} A\right) \subseteq \bigcap \gamma\left(B \downarrow_{c}\right.$ $A)+A=B *_{\gamma_{+}} A$, so $\bigcap \gamma\left(B \downarrow_{c} A\right) \subseteq B \cap\left(B *_{\gamma_{+}} A\right)$. For the other inclusion direction, it follows from +inclusion that $B \cap\left(B *_{\gamma^{+}} A\right)=B \cap\left(\bigcap \gamma\left(B \downarrow_{c} A\right)+A\right) \subseteq B \cap\left(\bigcap \gamma\left(B \downarrow_{c} A\right) \cup A\right)$, whence $\left.B \cap\left(B *_{\gamma_{+}} A\right) \subseteq\left(B \cap\left(\bigcap \gamma\left(B \downarrow_{c} A\right)\right)\right) \cup(B \cap A)\right)=\left(\cap \gamma\left(B \downarrow_{c} A\right)\right) \cup(B \cap A)$. If $B \cap A=\varnothing$, the inclusion follows directly. Otherwise, $B \forall \perp$ and by Observation 20 we have that $B \cap A \subseteq \bigcap \gamma\left(B \downarrow_{c} A\right)$. Thus, it follows that $B \cap\left(B *_{\gamma_{+}} A\right) \subseteq \bigcap \gamma\left(B \downarrow_{c} A\right)$ and, therefore, $B \cap\left(B *_{\gamma+} A\right)=\bigcap \gamma\left(B \downarrow_{c} A\right)$.

\section{Proof of Observation 22}

Proof. It is easy to see that $\cap \gamma\left(\left(\cap \gamma\left(B \downarrow_{c} A\right)\right) \downarrow_{c} A\right)=\cap \gamma\left(B \downarrow_{c} A\right)$ for every $A$. So, $B *_{\gamma_{+}} A=$ $\cap \gamma\left(B \downarrow_{c} A\right)+A=\cap \gamma\left(\left(\cap \gamma\left(B \downarrow_{c} A\right)\right) \downarrow_{c} A\right)+A$. By Observation 21, $\cap \gamma\left(\left(\cap \gamma\left(B \downarrow_{c} A\right)\right) \downarrow_{c} A\right)+A=$ $\cap \gamma\left((B \cap(B * A)) \downarrow_{c} A\right)+A$. Then $B *_{\gamma_{+}} A=\cap \gamma\left(\left(B \cap\left(B *_{\gamma^{+}}\right) \downarrow_{c} A\right)+A=\left(B \cap\left(B *_{\gamma_{+}} A\right)\right) *_{\gamma_{+}} A\right.$. 
In order to show ${ }^{*}$-coincidence, let $B \cap A \neq \varnothing$ (which implies that $B \forall \perp$ ) and $A \subseteq C \subseteq B \cup A$. It follows that $B \cap C \neq \varnothing$. Since $B \Vdash \perp$, by Observation 18 and the definition of $\gamma$ we have that $\cap \gamma\left(B \downarrow_{c} A\right)=B=\cap \gamma\left(B \downarrow_{c} C\right)$. Then, by $\dot{+}$-coincidence we know that $B+A=B+C$ and, therefore, $B{ }_{\gamma+} A=B{ }_{\gamma_{\gamma}} C$.

The following lemma will be useful in the proof of the representation theorem:

Lemma 6. Let $*$ be an operator on B that satisfies ${ }^{*}$-relevance and $\gamma$ be a function such that, for every non-empty, consistent set $A$, it holds that:

- $\gamma\left(B \downarrow_{c} A\right)=B$ whenever $B \downarrow_{c} A=\varnothing$;

- $\gamma\left(B \downarrow_{c} A\right)=\left\{X \in B \downarrow_{c} A:(B \cap(B * A)) \subseteq X\right\}$ whenever $B \downarrow_{c} A \neq \varnothing$.

Then $B \cap(B * A)=\cap \gamma\left(B \downarrow_{c} A\right)$.

Proof. That $B \cap(B * A) \subseteq \cap \gamma\left(B \downarrow_{c} A\right)$ is trivial by the definition of $\gamma$. For the other inclusion direction, suppose towards contradiction that $\cap \gamma\left(B \downarrow_{c} A\right) \nsubseteq B \cap(B * A)$. So there exists $\varphi \in \cap \gamma\left(B \downarrow_{c} A\right)$ such that $\varphi \notin B \cap(B * A)$. From the definitions of $\downarrow_{c}$ and $\gamma$, we have that $\varphi \in B$ and, hence, $\varphi \in B \backslash B * A$. By $*_{c}$-relevance, there is some $B^{\prime}$ with $B \cap\left(B *_{c} A\right) \subseteq B^{\prime} \subseteq B$, such that $B^{\prime} \cup\{\psi\}$ is consistent for some $\psi \in A$ and $B^{\prime} \cup\{\varphi\} \cup\{\lambda\}$ is inconsistent for every $\lambda \in A$. So we may infer that $\varphi \notin B^{\prime}$. Then we may extend $B^{\prime}$ to a maximal subset $B^{\prime \prime}$ of $B$ consistent with some $\alpha \in A$. From ${ }^{*}$-relevance we know that $\varphi \notin B^{\prime \prime}, B \cap(B * A) \subseteq B^{\prime \prime}$ and, by the definition of $\gamma, B^{\prime \prime} \in \gamma\left(B \downarrow_{c} A\right)$. But since $\varphi \in \cap \gamma\left(B \downarrow_{c} A\right)$, for every $X \in \gamma\left(B \downarrow_{c} A\right)$ it holds that $\varphi \in X$, which is a contradiction. Then $\cap \gamma\left(B \downarrow_{c} A\right) \subseteq B \cap(B * A)$ and, therefore, $B \cap(B * A)=\cap \gamma\left(B \downarrow_{c} A\right)$.

\section{Proof of Theorem 66}

Proof. (Construction to postulates): let $\gamma$ be an arbitrary selection function for $B,+$ be a consistency-preserving partial expansion operator for $B$ and ${ }_{\gamma_{+}+}$be the multiple choice partial meet revision on $B$ generated by $\gamma$ and + . Then, for all sets $A$ :

$$
B *_{\gamma+} A= \begin{cases}\bigcap_{B} \gamma\left(B \downarrow_{c} A\right)+A & \text { if } A \neq \varnothing \text { and } A H \perp \\ B & \text { otherwise }\end{cases}
$$

We must show that ${ }^{*}{ }_{\gamma+}$ satisfies all the enumerated postulates:

(Inclusion) Since every $X \subseteq B \downarrow_{c} A$ is such that $X \subseteq B, \gamma\left(B \downarrow_{c} A\right) \subseteq B \downarrow_{c} A$ (when $\left.B \downarrow_{c} A \neq \varnothing\right)$ or $\gamma\left(B \downarrow_{c} A\right)=\{B\}$ (when $B \downarrow_{c} A$ is empty) and + satisfies inclusion, this postulate is trivially shown.

(Weak consistency) Suppose that $A$ is consistent. By the definition of remainder set, every $X \in B \downarrow_{c} A$ is consistent with some $\alpha \in A$. Therefore, the intersection of any subset of $B \downarrow_{c} A$ is consistent with some $\alpha \in A$. Then, by $\dot{+}$-consistency it follows that $B{ }^{\gamma_{+}} A$ is consistent.

(Weak success) Suppose that $A \neq \varnothing$ and $A H \perp$. Then, by the definition of ${ }^{*}{ }_{+}$and by $\dot{+}$-success, it follows that $A \cap B{ }_{\gamma_{+}} A \neq \varnothing$. 
(Vacuity 1) Trivial by definition.

(Uniformity 2) Assume that $\mathrm{A}$ and $\mathrm{C}$ are consistent bases and that it holds for all $B^{\prime} \subseteq B$ that $B^{\prime} \cup\{\alpha\}$ is consistent for some $\alpha \in A$ iff $B^{\prime} \cup\{\psi\}$ is consistent for some $\psi \in C$. By Observation 21, $B \cap\left(B *_{\gamma_{+}} A\right)=\bigcap \gamma\left(B \downarrow_{c} A\right)$ (and analogously the same for $C$ ). So it is sufficient to show that $\bigcap \gamma\left(B \downarrow_{c} A\right)=\bigcap \gamma\left(B \downarrow_{c} C\right)$. From the initial assumption and Observation 19, we can infer that $B \downarrow_{c} A=B \downarrow_{c} C$. Since $\gamma$ is a well-defined function, then $\gamma\left(B \downarrow_{c} A\right)=\gamma\left(B \downarrow_{c} C\right)$. So, $B \cap\left(B *_{\gamma_{+}} A\right)=B \cap\left(B *_{\gamma_{+}} C\right)$.

(Relevance) Let $\varphi \in B \backslash B{ }^{*}{ }_{c} A$. Then, by $\dot{+}$-preservation, $\bigcap \gamma\left(B \downarrow_{c} A\right) \neq B$, i.e., $B \downarrow_{c} A \neq \varnothing$. So there exists $X \in \gamma\left(B \downarrow_{c} A\right)$ such that $\bigcap \gamma\left(B \downarrow_{c} A\right) \subseteq X$ and $\varphi \notin X$. By Observation $21, B \cap\left(B *_{c} A\right)=\bigcap \gamma\left(B \downarrow_{c} A\right)$, so it follows from $\bigcap \gamma\left(B \downarrow_{c} A\right) \subseteq X$ that $B \cap\left(B *_{c} A\right) \subseteq X$. Moreover, it follows from $X \in \gamma\left(B \downarrow_{c} A\right)$ and $\varphi \in B \backslash X$ that $X \cup\{\psi\}$ is consistent for some $\psi \in A$ and $X \cup\{\varphi\} \cup\{\lambda\}$ is inconsistent for any $\lambda \in A$. Thus, $X$ is the set we are looking for.

(Postulates to construction): let $B$ be a belief base and $*$ be an operator on $B$ that satisfies the enumerated postulates. We must show that $*$ is a multiple choice partial meet revision. Let $\gamma$ be a function such that, for every non-empty, consistent set $A$, it holds that:

- $\gamma\left(B \downarrow_{c} A\right)=B$ whenever $B \downarrow_{c} A=\varnothing$;

- $\gamma\left(B \downarrow_{c} A\right)=\left\{X \in B \downarrow_{c} A:(B \cap(B * A)) \subseteq X\right\}$ whenever $B \downarrow_{c} A \neq \varnothing$.

Let + be an operator on $B$ such that, for every set $A, \cap \gamma\left(B \downarrow_{c} A\right)+A=\cap \gamma\left(B \downarrow_{c}\right.$ $A) \cup(A \cap(B * A))$.

We must show:

(Part A)

1. $\gamma$ is a well-defined function. Suppose that $B \downarrow_{c} A=B \downarrow_{c} C$ for two consistent sets $A$ and $C$. It follows from ${ }^{*}$-uniformity 2 that $B \cap(B * A)=B \cap(B * C)$. Hence, $\gamma\left(B \downarrow_{c} A\right)=\gamma\left(B \downarrow_{c} C\right)$.

2. If $B \downarrow_{c} A=\varnothing$ we must show that $\gamma\left(B \downarrow_{c} A\right)=B$. It follows from the definition of $\gamma$.

3. If $B \downarrow_{c} A \neq \varnothing$ we must show that $\varnothing \neq \gamma\left(B \downarrow_{c} A\right) \subseteq B \downarrow_{c} A$. It follows from the definition of $\gamma$ that $\gamma\left(B \downarrow_{c} A\right) \subseteq B \downarrow_{c} A$. Now we prove that $\gamma\left(B \downarrow_{c} A\right) \neq \varnothing$. By ${ }^{*}{ }_{c}$-weak success it holds that $A \cap(B * A) \neq \varnothing$. Let $\alpha \in A \cap(B * A)$. Then we have that $(B \cap(B * A)) \cup\{\alpha\} \subseteq B * A$. It follows from ${ }^{*}$-weak consistency that $B * A$ is consistent and, therefore, we can conclude that $(B \cap(B * A)) \cup\{\alpha\}$ is also consistent. Hence, there must be some $X \in B \downarrow_{c} A$ such that $B \cap(B * A) \subseteq X$. Thus, $\gamma\left(B \downarrow_{c} A\right) \neq \varnothing$.

$($ Part B $)+$ is a partial expansion operation. For that, it is enough to show that it satisfies the postulates from Theorem 35:

- $\dot{+}$-inclusion and $\dot{+}$-preservation follow directly from the definition of the operator.

- Since * satisfies $*_{c}$-weak success, + satisfies $\dot{+}$-success.

- In order to show $\dot{+}$-coincidence, assume that $\left(\cap \gamma\left(B \downarrow_{c} A\right)\right) \cap A \neq \varnothing$ (which implies that $B \cap A \neq \varnothing)$ and $A \subseteq C \subseteq\left(\cap \gamma\left(B \downarrow_{c} A\right) \cup A\right)$. We have to show that (I) $\cap \gamma\left(B \downarrow_{c}\right.$ $A)+A=\cap \gamma\left(B \downarrow_{c} A\right)+C(\mathrm{II}) . \cap \gamma\left(B \downarrow_{c} A\right)+A=\cap \gamma\left(B \downarrow_{c} A\right) \cup(A \cap(B * A))$ and 
$\cap \gamma\left(B \downarrow_{c} A\right)+C=\cap \gamma\left(B \downarrow_{c} A\right) \cup(C \cap(B * A))$. Between (I) and (II) the first terms of the unions are equal. So it remains to check the second ones. Since $A \subseteq C$, $(A \cap(B * A)) \subseteq(C \cap(B * A))$. Suppose that $(C \cap(B * A)) \nsubseteq A \cap(B * A)$. Then, there exists $\varphi \in C$ such that $\varphi \in(B * A)$ and $\varphi \notin A$. By $*_{c}$-inclusion we have that $\varphi \in B$. From $B \cap A \neq \varnothing$ and Observation 18 we have that $B \downarrow_{c} A=\{B\}$ and, from the definition of $\gamma, \gamma\left(B \downarrow_{c} A\right)=B$ (since $\left.B \cap(B * A) \subseteq B\right)$. Hence, if $C \cap(B * A)$ has an element that is not in $A \cap(B * A)$, then it is in the first term of the union. Therefore, (I) $=($ II $)$ and + -coincidence is satisfied.

- In order to show $\dot{+}$-consistency, we must show that $\cap \gamma\left(B \downarrow_{c} A\right)+A$ is consistent when possible. Assume that there exists $X$ such that $\cap \gamma\left(B \downarrow_{c} A\right) \subseteq X \subseteq \cap \gamma\left(B \downarrow_{c} A\right) \cup A$ and $X \forall \perp$. We need to show that $\cap \gamma\left(B \downarrow_{c} A\right)+A H \perp$. From Lemma 6 we have that $\cap \gamma\left(B \downarrow_{c} A\right)=B \cap(B * A)$. Then, $\cap \gamma\left(B \downarrow_{c} A\right)+A=B \cap(B * A) \cup(A \cap(B * A))$. From the assumption about $X$ we have that $B \cap(B * A) \nvdash \perp$. If $A \vdash \perp$, by $*_{c}$-vacuity 1 we know that $B * A=B$ and we are done. If $A \forall \perp$, from ${ }^{*}$-weak consistency we know that $B * A \nvdash \perp$ and, since $(B \cap(B * A)) \cup(A \cap(B * A)) \subseteq B * A, \cap \gamma\left(B \downarrow_{c} A\right)+A \nvdash \perp$. Therefore, $+\dot{+}$-consistency is satisfied.

(Part C). ${ }^{*}{ }_{\gamma^{+}}$is equal to *, that is, $B{ }^{*}{ }_{\gamma_{+}} A=B * A$, for any set $A$. If $A$ is empty or inconsistent, by ${ }^{*}$-vacuity 1 the equality holds. Suppose that $A$ is consistent:

1. We want to show that $B * A \subseteq B *_{\gamma_{+}} A$. Let $\alpha \in B * A$. We must show that $\alpha \in B{ }_{\gamma_{+}} A$. We have two cases:

- $\alpha \notin A$. By * ${ }_{c}$-inclusion it holds that $B * A \subseteq B \cup A$ and, then, $\alpha \in B$. Therefore, $\alpha \in B \cap(B * A)$. Hence, it follows from the definition of $\gamma$ that $\alpha \in X$ for all $X \in \gamma\left(B \downarrow_{c} A\right)$ and we are done.

- $\alpha \in A$. As $\alpha \in B * A, \alpha \in A \cap(B * A)$ and, by the definition of $\dot{+}, \alpha \in B *_{\gamma_{+}} A$.

2. We want to show that $B{ }_{\gamma_{+}} A \subseteq B * A$. Let $\alpha \notin B * A$. We must show that $\alpha \notin B{ }_{\gamma_{+}} A$. For that, we need to find some $X \in\left(B \downarrow_{c} A\right)$ such that $\alpha \notin X$. We have two cases:

- $\alpha \in B$. By ${ }^{*}$-relevance, there exists some $B^{\prime}$ with $B \cap(B * A) \subseteq B^{\prime} \subseteq B$ such that $B^{\prime} \cup\{\psi\}$ is consistent for some $\psi \in A$ and $B^{\prime} \cup\{\alpha\} \cup\{\lambda\}$ is inconsistent for every $\lambda \in A$. So we may infer that $\alpha \notin B^{\prime}$. Then we may extend $B^{\prime}$ to a maximal subset $X$ of $B$ consistent with some $\varphi \in A$ such that $B \cap(B * A) \subseteq X$ and $\alpha \notin X$. Hence, $X \in \gamma\left(B \downarrow_{c} A\right)$ and $\alpha \notin X$.

- $\alpha \notin B$. Then no set in $B \downarrow_{c} A$ will contain $\alpha$.

\section{Proof of Observation 23}

Proof. If there exists $\psi \in A$ such that $B \cup\{\psi\} \forall \perp$, then the second condition of Definition 96 will never be satisfied, given that for any $X \subseteq B$ it is impossible that $\forall \lambda \in A, X \cup\{\lambda\} \vdash \perp$. If $B \forall \perp$ and $B \cap A \neq \varnothing$, it follows directly that there exists $\psi \in A$ such that $B \cup\{\psi\} \forall \perp$ $(\psi \in A \cap B)$. Therefore, in both cases we have that $B \downarrow_{c} A=\varnothing$. 


\section{Proof of Observation 24}

Proof. From Observation 23 we have that $B \downarrow_{c} A=\varnothing$. Since $A \subseteq C \subseteq(A \cup B), C \cap B \neq \varnothing$ and, analogously, $B \downarrow_{c} C=\varnothing$. Therefore, $B \downarrow_{c} A=B \downarrow_{c} C$.

\section{Proof of Observation 25}

Proof. It follows directly from Observation 23.

\section{Proof of Observation 26}

Proof. By the definitions of $B \downarrow_{c} A$ and $\sigma$ and by $\dot{+}$-preservation, $B \backslash \sigma\left(B \downarrow_{c} A\right) \subseteq B$ and $B \backslash \sigma\left(B \downarrow_{c} A\right) \subseteq B \backslash \sigma\left(B \downarrow_{c} A\right)+A=B *_{\sigma+} A$, so $B \backslash \sigma\left(B \downarrow_{c} A\right) \subseteq B \cap\left(B *_{\sigma+} A\right)$. For the other inclusion direction, we have that $B \cap\left(B *_{\sigma+} A\right)=B \cap\left(\left(B \backslash \sigma\left(B \downarrow_{c} A\right)\right)+A\right)$ and it follows from $\dot{+}$-inclusion that $B \cap\left(\left(B \backslash \sigma\left(B \downarrow_{c} A\right)\right)+A\right) \subseteq B \cap\left(\left(B \backslash \sigma\left(B \downarrow_{c} A\right)\right) \cup A\right)$, whence $B \cap\left(B *_{\sigma+} A\right) \subseteq\left(B \cap\left(B \backslash \sigma\left(B \downarrow_{c} A\right)\right) \cup(B \cap A)=\left(\left(B \backslash \sigma\left(B \downarrow_{c} A\right)\right)\right) \cup(B \cap A)\right.$. If $B$ is consistent, by Observation 25 we have that $B \cap A \subseteq\left(B \backslash \sigma\left(B \Downarrow_{c} A\right)\right)$ and, hence, $B \cap\left(B *_{\sigma+} A\right) \subseteq\left(B \backslash \sigma\left(B \downarrow_{c} A\right)\right)$. If $B \cap A=\varnothing$, the same result follows directly. Thus, $B \cap\left(B *_{\sigma+} A\right)=\left(B \backslash \sigma\left(B \downarrow_{c} A\right)\right)$.

\section{Proof of Observation 27}

Proof. If $A$ is inconsistent, then $B *_{\sigma+} A=B$ and the postulate follows trivially. Now suppose that $A$ is consistent.

From the definition of negation-free kernel set (Definition 96), we have that $B \backslash \sigma\left(B \Downarrow_{c}\right.$ $A)$ is consistent with some $\psi \in A$ and, as a consequence, $\left(B \backslash \sigma\left(B \downarrow_{c} A\right)\right) \downarrow_{c} A=\varnothing$. Then, $B \backslash \sigma\left(B \Downarrow_{c} A\right)=\left(B \backslash \sigma\left(B \Downarrow_{c} A\right)\right) \backslash \sigma\left(\left(B \backslash \sigma\left(B \Downarrow_{c} A\right)\right) \Downarrow_{c} A\right)$ and $B *_{\sigma+} A=\left(B \backslash \sigma\left(B \Downarrow_{c}\right.\right.$ $A))+A=\left(B \backslash \sigma\left(B \downarrow_{c} A\right)\right) \backslash \sigma\left(\left(B \backslash \sigma\left(B \downarrow_{c} A\right)\right) \downarrow_{c} A\right)+A$. By Observation 26 we have that $\left(B \backslash \sigma\left(B \downarrow_{c} A\right)\right) \backslash \sigma\left(\left(B \backslash \sigma\left(B \downarrow_{c} A\right)\right) \downarrow_{c} A\right)+A=\left(B \cap\left(B *_{\sigma+} A\right)\right) \backslash \sigma\left(\left(B \cap\left(B *_{\sigma+} A\right)\right) \downarrow_{c}\right.$ $A)+A=\left(B \cap\left(B *_{\sigma+} A\right)\right) *_{\sigma+} A$. Therefore, $B *_{\sigma+} A=\left(B \cap\left(B *_{\sigma+} A\right)\right) *_{\sigma+} A$.

In order to show ${ }^{*}$-coincidence, let $B, A$ and $C$ be such that $A \cap B \neq \varnothing$ and $A \subseteq C \subseteq(A \cup B)$. We have to show that $B *_{c} A=B *_{c} C$. Since $B H \perp$ or $B \cap A=\varnothing$, it holds that $B H \perp$. If $A \vdash \perp$, then $B *_{\sigma+} A=B$. Since $A \subseteq C$, then $C \vdash \perp, B *_{\sigma+} C=B$ and we are done. If $A H \perp$, given that $A \cap B \neq \varnothing$ and $B H \perp$, we have that $B \downarrow_{c} A=\varnothing$. From $A \subseteq C$, we also have that $C \cap B \neq \varnothing$ and, then, $B \downarrow_{c} C=\varnothing$. Hence, $B *_{\sigma+} A=B \backslash\left(B \downarrow_{c} A\right)+A=B+A$ and $B *_{\sigma+} C=B \backslash \sigma\left(B \Downarrow_{c} C\right)+C=B+C$. By + -coincidence it holds that $B+A=B+C$. Therefore, $B *_{\sigma+} A=B *_{\sigma+} C$ and, then, ${ }^{*}{ }_{c}$-coincidence holds.

\section{Proof of Observation 28}

Proof. Let $X \subseteq B$ be such that $X \in B \downarrow_{c} A$. Suppose that $X \notin B \downarrow_{c} C$. From Definition 96, the only possible reason for that is $X$ not being minimal: $\exists X^{\prime} \subset X$ such that $X^{\prime} \in B \downarrow_{c} C$. As a consequence, $X^{\prime} \cup\{\alpha\}$ is inconsistent for every $\alpha \in A$ and $X \notin B \downarrow_{c} A$, since $X$ is not minimal. Contradiction. Then, if $X \in B \Downarrow_{c} A$, then $X \in B \Downarrow_{c} C$. The converse property is analogous. Therefore, $B \downarrow_{c} A=B \downarrow_{c} C$. 
The following lemma will be useful in the proof of the representation theorem:

Lemma 7. Let * be an operator on $B$ and $\sigma$ be a function such that, for every set $A$, it holds that $\sigma\left(B \downarrow_{c} A\right)=\{\alpha: \alpha \in(B \backslash(B * A))\}$. Then $B \cap(B * A)=B \backslash \sigma\left(B \downarrow_{c} A\right)$.

Proof. Since $\sigma\left(B \downarrow_{c} A\right)=B \backslash(B * A)$, then $B \backslash \sigma\left(B \downarrow_{c} A\right)=B \backslash(B \backslash(B * A))=B \cap(B * A)$.

\section{Proof of Theorem 67}

Proof. (Construction to postulates): let $\sigma$ be an arbitrary incision function for $B,+$ be a consistency-preserving partial expansion operator for $B$ and $*_{\sigma+}$ be the multiple choice

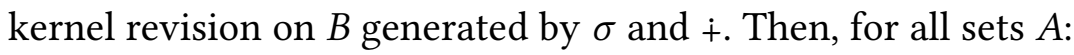

$$
B *_{\sigma+} A= \begin{cases}\left(B \backslash \sigma\left(B \downarrow_{c} A\right)\right)+A & \text { if } A \neq \varnothing \text { and } A \forall \perp \\ B & \text { otherwise }\end{cases}
$$

We must show that $*_{\sigma+}$ satisfies all the enumerated postulates.

(Inclusion) Since $\left(B \backslash \sigma\left(B \downarrow_{c} A\right)\right) \subseteq B$ and + satisfies inclusion, this postulate is trivially shown.

(Weak consistency) Suppose that $A$ is consistent. Since $\sigma\left(B \downarrow_{c} A\right)$ cuts every subset of $B$ that is minimally inconsistent with any $\lambda \in A$, then $B \backslash \sigma\left(B \downarrow_{c} A\right)$ is consistent with some $\psi \in A$. Therefore, from $\dot{+}$-consistency it follows that $B{ }^{*} \sigma+A$ is consistent.

(Weak success) Suppose that $A \neq \varnothing$ and $A \Vdash \perp$. Then, by the definition of ${ }^{*}{ }_{\sigma+}$ and by $\dot{+}$-success, it is trivial that $A \cap B *_{\sigma+} \neq \varnothing$.

(Vacuity 1) Trivial by definition.

(Uniformity 1) Assume that $A$ and $C$ are consistent bases and that it holds for all $B^{\prime} \subseteq B$ that $B^{\prime} \cup\{\alpha\}$ is inconsistent for every $\alpha \in A$ iff $B^{\prime} \cup\{\psi\}$ is inconsistent for every $\psi \in C$. By Observation $26, B \cap\left(B *_{\sigma+} A\right)=\left(B \backslash \sigma\left(B \downarrow_{c} A\right)\right.$ ) (and analogously the same for $C)$. So it remains to show that $\left(B \backslash \sigma\left(B \downarrow_{c} A\right)\right)=\left(B \backslash \sigma\left(B \downarrow_{c} C\right)\right)$. From the initial assumption and Observation 28, we can infer that $B \downarrow_{c} A=B \downarrow_{c} C$. Since $\sigma$ is a well-defined function, then $\sigma\left(B \downarrow_{c} A\right)=\sigma\left(B \downarrow_{c} C\right)$. So, $B \backslash\left(B *_{\sigma+} A\right)=B \backslash\left(B *_{\sigma+} C\right)$.

(Core-retainment) Let $\varphi \in B \backslash B *_{\sigma+} A$. Then $B \backslash \sigma\left(B \downarrow_{c} A\right) \neq B$, i.e., $\sigma\left(B \downarrow_{c} A\right) \neq \varnothing$. So there exists $X \in\left(B \downarrow_{c} A\right)$ such that $\varphi \in X$ and $\varphi \in X \cap \sigma\left(B \downarrow_{c} A\right)$. It follows from $X \in\left(B \Downarrow_{c} A\right)$ and $\varphi \in X$ that $X$ is inconsistent for every $\lambda \in A$ and $X \backslash\{\varphi\} \cup\{\psi\}$ is consistent for some $\psi \in A$. Thus, $X \backslash\{\varphi\}$ is the set we are looking for.

(Postulates to construction): let $B$ be a belief base and $*$ be an operator on $B$ that satisfies the enumerated postulates. We must show that $*$ is a multiple choice kernel revision. Let $\sigma$ be a function such that, for every base $B$ and every non-empty, consistent set $A$, it holds that:

$$
\sigma\left(B \downarrow_{c} A\right)=\{\alpha: \alpha \in(B \backslash(B * A))\}
$$


Let + be an operator on $B$ such that, for every set $A,\left(B \backslash \sigma\left(B \Downarrow_{c} A\right)\right)+A=\left(B \backslash \sigma\left(B \downarrow_{c}\right.\right.$ $A)) \cup(A \cap(B * A))$.

We must show:

(Part A)

1. $\sigma$ is a well-defined function. Given two consistent sets $A$ and $C$, suppose that $B \downarrow_{c}$ $A=B \Downarrow_{c} C$. It follows from $*_{c}$-uniformity 1 that $B \backslash(B * A)=B \backslash(B * C)$. Therefore, $\sigma\left(B \downarrow_{c} A\right)=\sigma\left(B \downarrow_{c} C\right)$.

2. $\sigma\left(B \downarrow_{c} A\right) \subseteq \bigcup\left(B \downarrow_{c} A\right)$. Let $\varphi \in \sigma\left(B \downarrow_{c} A\right)$. Then $\varphi \in B \backslash(B * A)$. Due to $*_{c}$-core-retainment, there is some $B^{\prime} \subseteq B$ such that $B^{\prime} \cup\{\psi\}$ is consistent for some $\psi \in A$ and $B^{\prime} \cup\{\varphi\} \cup\{\lambda\}$ is inconsistent for every $\lambda \in A$. Now let $X$ be an arbitrary element of $\left(B^{\prime} \cup\{\varphi\}\right) \Downarrow_{c} A$. (Observe that by the conditions above it follows that $\left.\left(B^{\prime} \cup\{\varphi\}\right) \downarrow_{c} A \neq \varnothing\right)$. Then $X$ is such that $X \subseteq B^{\prime} \cup\{\varphi\}, X \cup\{\alpha\}$ is inconsistent for every $\alpha \in A$ and, for any $X^{\prime} \subset X$, it holds that $X^{\prime} \cup\{\beta\}$ is inconsistent for every $\beta \in A$. It follows that $\varphi \in X$ and that $X \cap A=\varnothing$. Therefore, $X \in B \Downarrow_{c} A$ and, since $\alpha \in X$, we can conclude that $\varphi \in \bigcup\left(B \downarrow_{c} A\right)$.

3. If $X \in B \downarrow_{c} A$ then $X \cap \sigma\left(B \downarrow_{c} A\right) \neq \varnothing$. Let $X \in B \downarrow_{c} A$. We need to show that $X \cap \sigma\left(B \Downarrow_{c} A\right) \neq \varnothing$. Suppose that $A \neq \varnothing$ and $A \forall \perp$. From $*_{c}$-weak consistency we have that $B * A$ is consistent. Since $X \cup\{\lambda\}$ is inconsistent for every $\lambda \in A$ and by ${ }^{*}$-weak success we know that $A \cap(B * A) \neq \varnothing$, we can infer that $X \nsubseteq B * A$. Hence, there is some $\beta \in X$ such that $\beta \notin B * A$. Therefore, $\beta \in \sigma\left(B \downarrow_{c} A\right)$.

(Part B) + is a weak partial expansion operation. For that, it is enough to show that it satisfies the postulates from Theorem 35:

- $\dot{+}$-inclusion and $\dot{+}$-preservation follow directly from the definition of the operator.

- Since * satisfies ${ }^{*}$-weak success, $\dot{+}$ satisfies $\dot{+}-$ weak success.

- In order to show $\dot{+}$-coincidence, let $B, A$ and $C$ be such that $\left(B \backslash \sigma\left(B \downarrow_{c} A\right)\right) \cap A \neq \varnothing$ and $A \subseteq C \subseteq\left(B \backslash \sigma\left(B \downarrow_{c} A\right)\right)$. From the definition of $\sigma$, we have that $\left(B \backslash \sigma\left(B \downarrow_{c}\right.\right.$ $A))=B \cap(B * A)$. So, we have to show that (I) $(B \cap(B * A))+A=(B \cap(B * A))+C$ (II). $(B \cap(B * A))+A=(B \cap(B * A)) \cup(A \cap(B * A))$ and $(B \cap(B * C))+C=(B \cap(B *$ $A)) \cup(C \cap(B * A))$. Between (I) and (II) the first terms of the unions are equal. So it remains to check the second ones. Since $A \subseteq C,(A \cap(B * A)) \subseteq(C \cap(B * A))$. Suppose that $(C \cap(B * A)) \nsubseteq A \cap(B * A)$. Then, there exists $\varphi \in C$ such that $\varphi \in(B * A)$ and $\varphi \notin A$. By ${ }^{*}$-inclusion we have that $\varphi \in B$. So, $\varphi \in B \cap(B * A)$. Hence, if $C \cap(B * A)$ has an element that is not in $A \cap(B * A)$, then it is in the first term of the union. Therefore, $(\mathrm{I})=(\mathrm{II})$ and $\dot{+}$-coincidence is satisfied.

- In order to show $\dot{+}$-consistency, we must show that $\left(B \backslash \sigma\left(B \downarrow_{c} A\right)\right)+A$ is consistent when possible. Assume that there exists $X$ such that $\left(B \backslash \sigma\left(B \Downarrow_{c} A\right)\right) \subseteq X \subseteq\left(B \backslash \sigma\left(B \downarrow_{c}\right.\right.$ $A)) \cup A$ and $X \forall \perp$. We must show that $\left(B \backslash \sigma\left(B \downarrow_{c} A\right)\right)+A H \perp$. From Lemma 7 we have that $B \backslash \sigma\left(B \downarrow_{c} A\right)=B \cap(B * A)$. Then, $\left(B \backslash \sigma\left(B \downarrow_{c} A\right)\right)+A=(B \cap(B * A)) \cup(A \cap(B * A))$. From the assumption we have that $B \cap(B * A) \forall \perp$. If $A \vdash \perp$, by ${ }^{*}$-vacuity 1 we know that $B * A=B$ and we are done. If $A \forall \perp$, from $*_{c}$-weak consistency we know that $B * A \forall \perp$ and, since $(B \cap(B * A)) \cup(A \cap(B * A)) \subseteq B * A,\left(B \backslash \sigma\left(B \downarrow_{c} A\right)\right)+A \forall \perp$. 
Therefore, $\dot{+}$-consistency is satisfied.

(Part C). $*_{\sigma+}$ is equal to *, that is, $B *_{\sigma+} A=B * A$, for any set $A$. If $A$ is empty or inconsistent, by ${ }^{*}$-vacuity 1 the equality holds. Suppose that $A$ is consistent:

1. We want to show that $B * A \subseteq B *_{\sigma+} A$. Let $\alpha \in B * A$. We must show that $\alpha \in B *_{\sigma+} A$. We have two cases:

- $\alpha \notin A$. By inclusion it holds that $B * A \subseteq B \cup A$ and, then, $\alpha \in B$. Therefore, $\alpha \notin B \backslash(B * A)$ and, hence, it follows from the definition of $\sigma$ that $\alpha \notin \sigma\left(B \downarrow_{c} A\right)$. By the definition of $*_{\sigma+}$ and by $\dot{+}$-preservation we have that $\alpha \in B *_{\sigma+} A$.

- $\alpha \in A$. As $\alpha \in B * A, \alpha \in A \cap(B * A)$ and, by the definition of $\dot{+}, \alpha \in B *_{\sigma+} A$.

2. We want to show that $B *_{\sigma+} A \subseteq B * A$. Let $\alpha \notin B * A$. We must show that $\alpha \notin B *_{\sigma+} A$. For that, it is necessary that $\alpha \in \sigma\left(B \downarrow_{c} A\right)$. We have two cases:

- $\alpha \in B$. Then, $\alpha \in(B \backslash B * A)$ and, consequently, $\alpha \in \sigma\left(B \downarrow_{c} A\right)$. Since $\alpha \notin A \cap(B * A)$, we have that $\alpha \notin B *_{\sigma+} A$.

- $\alpha \notin B$. Then $\alpha \notin B \backslash \sigma\left(B \downarrow_{c} A\right)$. Since $\alpha \notin A \cap(B * A)$ as well, we conclude that $\alpha \notin B *_{\sigma+} A$.

\section{Proof of Observation 29}

Proof. Let $B, A$ be consistent belief bases and suppose that $B \cup A$ is consistent and that $\dot{+}$ is a maximal partial expansion. Then $B \downarrow_{c} A=\varnothing=\sigma\left(B \downarrow_{c} A\right)$ and $B *_{c} A=B+A$. Since + satisfies maximal expansion, from the initial assumption we have that $B{ }^{*}{ }_{c} A=B \cup A$. Therefore, ${ }^{*}$-vacuity 3 is satisfied.

For partial meet, from the inicial assumptions we have that $B \downarrow_{c} A=\{B\}$ and $\cap \gamma\left(B \downarrow_{c}\right.$ $A)=B$. Hence, $B{ }^{*}{ }_{c} A=B+A$ and it follows the same way.

\section{Proof of Observation 30}

Proof. Trivial from Definition 99. 



\section{Bibliography}

[AGM85] Carlos Alchourrón, Peter Gärdenfors, and David Makinson. On the logic of theory change. Fournal of Symbolic Logic, 50(02):510-530, 1985.

[AM81] Carlos E Alchourrón and David Makinson. Hierarchies of regulations and their logic. In New studies in deontic logic, pages 125-148. Springer, 1981.

[AM82] Carlos Alchourrón and David Makinson. On the logic of theory change: Contraction functions and their associated revision functions. Theoria, 48(01):14-37, 1982.

[AM85] Carlos E Alchourrón and David Makinson. On the logic of theory change: Safe contraction. Studia logica, 44(4):405-422, 1985.

$\left[\mathrm{BCM}^{+}\right.$03] F. Baader, D. Calvanese, D.L. McGuinness, D. Nardi, and P.F. Patel-Schneider, editors. The Description Logic Handbook. Cambridge University Press, New York, NY, USA, 2003.

[BH01] Philippe Besnard and Anthony Hunter. A logic-based theory of deductive arguments. Artificial Intelligence, 128(1-2):203-235, 2001.

[BHLS17] Franz Baader, Ian Horrocks, Carsten Lutz, and Ulrike Sattler. An Introduction to Description Logic. Cambridge University Press, 2017.

[BK01] Kathleen Suzanne Barber and Joonoo Kim. Belief revision process based on trust: Agents evaluating reputation of information sources. In Trust in Cyber-societies, pages 73-82. Springer, 2001.

[Bou93] Craig Boutilier. Revision sequences and nested conditionals. In Proceedings of the Thirteenth International foint Conference on Artificial Intelligence, IFCAI 1993, France, pages 519-531. Morgan Kaufmann, 1993.

[Del08] James P. Delgrande. Horn clause belief change: Contraction functions. In Gerhard Brewka and Jérôme Lang, editors, Principles of Knowledge Representation and Reasoning: Proceedings of the Eleventh International Conference, KR 2008, Australia, pages 156-165. AAAI Press, 2008.

[Del11] James P. Delgrande. Revising by an inconsistent set of formulas. In Proceedings of the Twenty-Second International foint Conference on Artificial Intelligence, IfCAI 2011, Spain, fuly 16-22, 2011, pages 833-838. IJCAI/AAAI, 2011. 
[DG01] Aldo Franco Dragoni and Paolo Giorgini. Revising beliefs received from multiple sources. In Frontiers in belief revision, pages 429-442. Springer, 2001.

[DJ08] James P. Delgrande and Yi Jin. Parallel belief revision. In Dieter Fox and Carla P. Gomes, editors, Proceedings of the Twenty-Third AAAI Conference on Artificial Intelligence, AAAI 2008, USA, pages 430-435. AAAI Press, 2008.

[DJ12] James Delgrande and Yi Jin. Parallel belief revision: Revising by sets of formulas. Artificial Intelligence, 176(1):2223-2245, 2012.

[DP97] Adnan Darwiche and Judea Pearl. On the logic of iterated belief revision. Artificial Intelligence, 89(1-2):1-29, 1997.

[Dun95] Phan Minh Dung. On the acceptability of arguments and its fundamental role in nonmonotonic reasoning, logic programming and n-person games. Artificial intelligence, 77(2):321-357, 1995.

[FH94] André Fuhrmann and Sven Ove Hansson. A survey of multiple contractions. fournal of Logic, Language and Information, 3(1):39-75, 1994.

[FH99] Eduardo Fermé and Sven Ove Hansson. Selective revision. Studia Logica, 63(3):331-342, 1999.

[FH18] Eduardo Fermé and Sven Ove Hansson. Belief Change: Introduction and Overview. Springer Briefs in Computer Science Series. Springer, 2018.

[FKIRS12] M. Falappa, G. Kern-Isberner, M. Reis, and G. Simari. Prioritized and nonprioritized multiple change on belief bases. Fournal of Philosophical Logic, 41(1):77-113, 2012.

[FKIS02] Marcelo A Falappa, Gabriele Kern-Isberner, and Guillermo R Simari. Explanations, belief revision and defeasible reasoning. Artificial Intelligence, 141(1-2):1-28, 2002.

[FPA04] Giorgos Flouris, Dimitris Plexousakis, and Grigoris Antoniou. Generalizing the AGM postulates: preliminary results and applications. In NMR, pages 171-179, 2004.

[FPA05a] Giorgos Flouris, Dimitris Plexousakis, and Grigoris Antoniou. On applying the AGM theory to DLs and OWL. In International Semantic Web Conference, pages 216-231. Springer, 2005.

[FPA05b] Giorgos Flouris, Dimitris Plexousakis, and Grigoris Antoniou. Updating description logics using the AGM theory. In Proceedings of the 7th International Symposium on Logical Formalizations of Commonsense Reasoning, pages 69-76, 2005.

[FSS03] Eduardo Fermé, Karina Saez, and Pablo Sanz. Multiple kernel contraction. Studia Logica, 73(2):183-195, 2003.

[Fuh88] André Fuhrmann. Relevant Logics, Modal Logics and Theory Change. PhD thesis, Australian National University, Camberra, 1988. 
BIBLIOGRAPHY

[Fuh89] André Fuhrmann. Reflective modalities and theory change. Synthese, 81(1):115134, 1989.

[Fuh97] André Fuhrmann. An Essay on Contraction. CSLI Publications Stanford, 1997.

[Gär81] Peter Gärdenfors. An epistemic approach to conditionals. American Philosophical Quarterly, 18(3):203-211, 1981.

[Gär82] Peter Gärdenfors. Rules for rational changes of belief. Philosophical Essays dedicated to Lennart Aqvist on his fiftieth birthday, 34, 1982.

[Gär84] Peter Gärdenfors. Epistemic importance and minimal changes of belief. Australasian fournal of Philosophy, 62(2):136-157, 1984.

[Gär88] Peter Gärdenfors. Knowledge in Flux - Modeling the Dynamics of Epistemic States. MIT Press, 1988.

[Gar21] Marco Garapa. Selective base revisions. Journal of Philosophical Logic, pages $1-26,2021$.

[GFR18] Marco Garapa, Eduardo Fermé, and Maurício D. Luís Reis. Studies in credibilitylimited base revision. In Principles of Knowledge Representation and Reasoning: Proceedings of the Sixteenth International Conference, KR 2018, USA, pages 240-247. AAAI Press, 2018.

[GM88] Peter Gärdenfors and David Makinson. Revisions of knowledge systems using epistemic entrenchment. In Proceedings of the 2nd conference on Theoretical aspects of reasoning about knowledge, pages 83-95. Morgan Kaufmann Publishers Inc., 1988.

[GR95] Peter Gärdenfors and Hans Rott. Belief revision. Computational Complexity, 63(6):35-132, 1995.

[Gro88] Adam Grove. Two modellings for theory change. Journal of philosophical logic, pages 157-170, 1988.

[Han89] Sven Ove Hansson. New operators for theory change. Theoria, 55(2):114-132, 1989.

[Han91] Sven Ove Hansson. Belief Base Dynamics. PhD thesis, Uppsala University, Uppsala, Suécia, 1991.

[Han92] Sven Ove Hansson. A dyadic representation of belief. In Peter Gärdenfors, editor, Belief Revision, volume 29 of Lecture Notes in Cambridge Tracts in Theoretical Computer Science, pages 89-121. Cambridge University Press, New York, 1992.

[Han93] Sven Ove Hansson. Reversing the Levi identity. Fournal of Philosophical Logic, 22(6):637-669, 1993.

[Han94] Sven Ove Hansson. Kernel contraction. Fournal of Symbolic Logic, 59(3):845859, 1994. 
[Han97] Sven Hansson. Semi-revision. Journal of Applied Non-Classical Logics, 7(12):151-175, 1997.

[Han99a] Sven Ove Hansson. A survey of non-prioritized belief revision. Erkenntnis, 50(2-3):413-427, 1999.

[Han99b] Sven Ove Hansson. A Textbook of Belief Dynamics - Theory Change and Database Updating. Applied Logic Series. Kluwer Academic Publishers, Dordrecht, 1999.

[Han06] Sven Ove Hansson. Logic of belief revision. The Stanford Encyclopedia of Philosophy, 2006.

[Han14a] Sven Ove Hansson. Descriptor revision. Studia Logica, 102(5):955-980, 2014.

[Han14b] Sven Ove Hansson. Relations of epistemic proximity for belief change. Artificial Intelligence, 217:76-91, 2014.

[Han17] Sven Ove Hansson. Descriptor Revision: Belief Change Through Direct Choice, volume 46 of Trends in Logic. Springer, 2017.

[Har76] William L Harper. Rational conceptual change. In PSA: Proceedings of the Biennial Meeting of the Philosophy of Science Association, volume 1976, pages 462-494. Philosophy of Science Association, 1976.

[HFCF01] Sven Ove Hansson, Eduardo Leopoldo Fermé, John Cantwell, and Marcelo Alejandro Falappa. Credibility limited revision. The Journal of Symbolic Logic, 66(4):1581-1596, 2001.

[HW02] Sven Ove Hansson and Renata Wassermann. Local change. Studia Logica, 70(1):49-76, 2002.

[KM91a] Hirofumi Katsuno and Alberto O Mendelzon. On the difference between updating a knowledge base and revising it. In Principles of Knowledge Representation and Reasoning: Proceedings of the Second International Conference, KR 1991, USA, page 387. Morgan Kaufmann Pub, 1991.

[KM91b] Hirofumi Katsuno and Alberto O Mendelzon. Propositional knowledge base revision and minimal change. Artificial Intelligence, 52(3):263-294, 1991.

[KP00] Sébastien Konieczny and Ramón Pino Pérez. A framework for iterated revision. Journal of Applied Non-classical logics, 10(3-4):339-367, 2000.

[KP02a] Sébastien Konieczny and Ramón Pino Pérez. Merging information under constraints: a logical framework. Fournal of Logic and computation, 12(5):773$808,2002$.

[KP02b] Sébastien Konieczny and Ramón Pino Pérez. Merging information under constraints: a logical framework. Fournal of Logic and computation, 12(5):773$808,2002$.

[KPHS07] Aditya Kalyanpur, Bijan Parsia, Matthew Horridge, and Evren Sirin. Finding all justifications of OWL DL entailments. In The Semantic Web, pages 267-280. Springer, 2007. 
BIBLIOGRAPHY

[KTF $\left.{ }^{+} 11\right]$ Patrick Krümpelmann, Matthias Thimm, Marcelo A Falappa, Alejandro J García, Gabriele Kern-Isberner, and Guillermo R Simari. Selective revision by deductive argumentation. In International Workshop on Theory and Applications of Formal Argumentation, pages 147-162. Springer, 2011.

[L $\left.\mathrm{L}^{+} 91\right] \quad$ Isaac Levi et al. The fixation of belief and its undoing: changing beliefs through inquiry. Cambridge University Press, 1991.

[Lev77] Isaac Levi. Subjunctives, dispositions and chances. Synthese, 34(4):423-455, 1977.

[Lev83] Isaac Levi. The enterprise of knowledge: An essay on knowledge, credal probability, and chance. MIT press, 1983.

[Lew73] David Lewis. Counterfactuals. Blackwell, Oxford, 1973.

[Lin91] Sten Lindström. A semantic approach to nonmonotonic reasoning: inference operations and choice. Uppsala Prints and Preprints in Philosophy, Department of Philosophy, University of Uppsala, 6, 1991.

[Mak85] David Makinson. How to give it up: A survey of some formal aspects of the logic of theory change. Synthese, 62(3):347-363, 1985.

[Mak87] David Makinson. On the status of the postulate of recovery in the logic of theory change. Journal of Philosophical Logic, 16(4):383-394, 1987.

[Mak97] David Makinson. Screened revision. Theoria, 63(1-2):14-23, 1997.

[MG91] David Makinson and Peter Gärdenfors. Relations between the logic of theory change and nonmonotonic logic. In The logic of theory change, pages 183-205. Springer, 1991.

[MGSW19] Vinícius Bitencourt Matos, Ricardo Guimarães, Yuri David Santos, and Renata Wassermann. Pseudo-contractions as gentle repairs. In Description Logic, Theory Combination, and All That, pages 385-403. Springer, 2019.

[ML14] Hua Meng and Sanjiang Li. A topological characterisation of belief revision over infinite propositional languages. In Pacific Rim International Conference on Artificial Intelligence, pages 77-90. Springer, 2014.

[ML15] H. Meng and T. Li. A topological characterization of multiple revision. In 2015 10th International Conference on Intelligent Systems and Knowledge Engineering (ISKE), pages 71-76, 2015.

[MLH00] Thomas Andreas Meyer, Willem Adrian Labuschagne, and Johannes Heidema. Refined epistemic entrenchment. Fournal of Logic, Language and Information, 9(2):237-259, 2000.

[Nay94] Abhaya C Nayak. Iterated belief change based on epistemic entrenchment. Erkenntnis, 41(3):353-390, 1994.

[Pep96] Pavlos Peppas. Well behaved and multiple belief revision. In 12th European Conference on Artificial Intelligence (ECAI 96), Hungary, pages 90-94. John Wiley and Sons, Chichester, 1996. 
[Pep04] Pavlos Peppas. The limit assumption and multiple revision. Fournal of Logic and Computation, 14(3):355-371, 2004.

[Pep08] Pavlos Peppas. Belief revision. In Handbook of Knowledge Representation, volume 3 of Foundations of Artificial Intelligence, pages 317-359. Elsevier, 2008.

[PKW12] Pavlos Peppas, Costas D. Koutras, and Mary-Anne Williams. Maps in multiple belief change. ACM Trans. Comput. Logic, 13(4):30:1-30:23, 2012.

[PSLS16] Carlos Eduardo Pantoja, Márcio Fernando Stabile, Nilson Mori Lazarin, and Jaime Simão Sichman. Argo: An extended Jason architecture that facilitates embedded robotic agents programming. In International Workshop on Engineering Multi-Agent Systems, pages 136-155. Springer, 2016.

[Rei11] Maurício Duarte Luís Reis. On theory multiple contraction. PhD thesis, Universidade da Madeira (Portugal), 2011.

[Res14] Fillipe Manoel Xavier Resina. Revisão de crenças em lógicas de descrição-um plug-in para o protégé. Master’s thesis, Universidade de São Paulo, 2014.

[RGW $\left.{ }^{+} 20\right]$ Fillipe Resina, Marco Garapa, Renata Wassermann, Eduardo Fermé, and Maurício D. Luís Reis. Choosing what to believe - new results in selective revision. In Principles of Knowledge Representation and Reasoning: Proceedings of the Seventeenth International Conference, KR 2020, Greece, pages 687-691, 2020.

[Rib10] Márcio Ribeiro. Revisão de Crenças em Lógicas de Descrição e em Outras Lógicas não Clássicas. PhD thesis, Universidade de São Paulo (USP), 2010.

[Rot01] Hans Rott. Change, choice and inference: A study of belief revision and nonmonotonic reasoning. Number 42. Clarendon Press, 2001.

[RW09] M. Ribeiro and R. Wassermann. Base revision for ontology debugging. fournal of Logic and Computation, 19(5):721-743, 2009.

[RW14] Márcio Moretto Ribeiro and Renata Wassermann. Minimal change in AGM revision for non-classical logics. In Principles of Knowledge Representation and Reasoning: Proceedings of the Fourteenth International Conference, KR 2014, Austria. AAAI Press, 2014.

[Spo10] Wolfgang Spohn. Multiple contraction revisited. In EPSA epistemology and methodology of science, pages 279-288. Springer, 2010.

[SPS18] Márcio Fernando Stabile, Carlos Eduardo Pantoja, and Jaime Simão Sichman. Experimental analysis of the effect of filtering perceptions in BDI agents. International fournal of Agent-Oriented Software Engineering, 6(3-4):329-368, 2018.

[TDG ${ }^{+}$19] Luciano H Tamargo, Cristhian AD Deagustini, Alejandro J García, Marcelo A Falappa, and Guillermo R Simari. Multi-source multiple change on belief bases. International fournal of Approximate Reasoning, 110:145-163, 2019. 
BIBLIOGRAPHY

[TGFS12] Luciano H Tamargo, Alejandro J García, Marcelo A Falappa, and Guillermo R Simari. Modeling knowledge dynamics in multi-agent systems based on informants. Knowledge Engineering Review, 27(1):87-114, 2012.

[VF16] Néstor Jorge Valdez and Marcelo Alejandro Falappa. Multiple revision on Horn belief bases. In XXII Congreso Argentino de Ciencias de la Computación (CACIC 2016)., 2016.

[YJW14] Yongfeng Yuan, Shier Ju, and Xuefeng Wen. Evaluative multiple revision based on core beliefs. Journal of Logic and Computation, 25(3):781-804, 2014.

[Yua17] Yongfeng Yuan. Rational metabolic revision based on core beliefs. Synthese, 194(6):2121-2146, 2017.

[ZCZC97] Dongmo Zhang, Shifu Chen, Wujia Zhu, and Zhaoqian Chen. Representation theorems for multiple belief changes. In Proceedings of the Fifteenth International Joint Conference on Artificial Intelligence, IFCAI 97, Japan, pages 89-94. Morgan Kaufmann, 1997.

[ZCZL97] Dongmo Zhang, Shifu Chen, Wujia Zhu, and Hongbing Li. Nonmonotonic reasoning and multiple belief revision. In Proceedings of the Fifteenth International Joint Conference on Artificial Intelligence, IFCAI 97, Japan, pages 95-101. Morgan Kaufmann, 1997.

[ZF01] Dongmo Zhang and Norman Foo. Infinitary belief revision. Fournal of Philosophical Logic, 30(6):525-570, 2001.

[ZH15] Li Zhang and Sven Ove Hansson. How to make up one's mind. Logic fournal of the IGPL, 23(4):705-717, 2015.

[Zha96] Dongmo Zhang. Belief revision by sets of sentences. Fournal of Computer Science and Technology, 11(2):108-125, 1996.

[Zha17] Li Zhang. Believability relations for select-direct sentential revision. Studia Logica, 105(1):37-63, 2017.

[Zha18] Li Zhang. Choice revision on belief bases. arXiv preprint arXiv:1805.01325, 2018.

[Zha19] Li Zhang. Choice revision. Journal of Logic, Language and Information, 28(4):577-599, 2019.

[ZPZ13] Zhi Qiang Zhuang, Maurice Pagnucco, and Yan Zhang. Definability of horn revision from horn contraction. In Proceedings of the Twenty-Third International foint Conference on Artificial Intelligence, IFCAI 2013, China, pages 1205-1212. IJCAI/AAAI, 2013. 